BBAREV XS4(X)

\title{
Cardiolipins and biomembrane function
}

\author{
Frederic L. Hoch
}

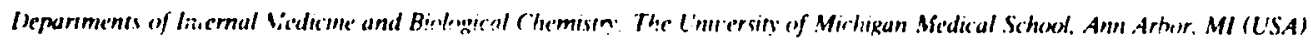

(Received 25 March 1991)

\section{Contents}

I. Introduction $\ldots \ldots \ldots \ldots \ldots \ldots \ldots \ldots \ldots \ldots \ldots \ldots \ldots \ldots \ldots \ldots$

11. Phospholinids and membrane energy transduction $\ldots \ldots \ldots \ldots \ldots \ldots \ldots \ldots$

A. Statc 1 respiration: promon leak $\ldots \ldots \ldots \ldots \ldots \ldots \ldots \ldots \ldots \ldots \ldots \ldots \ldots \ldots$

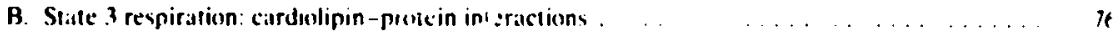

Ili. Casdiclipins in prokaryots:s $\ldots \ldots \ldots \ldots \ldots \ldots \ldots \ldots \ldots \ldots$

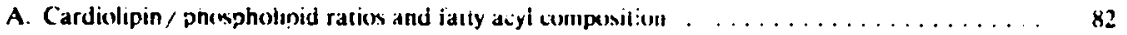

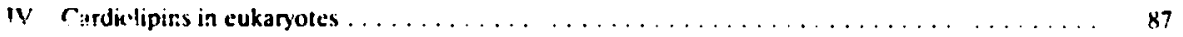

A. Cardiolipin / phospholipid atios and fatty acyl composition $\ldots \ldots \ldots \ldots \ldots \ldots$

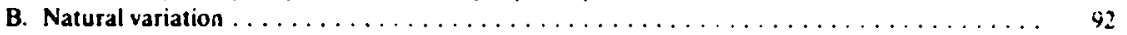

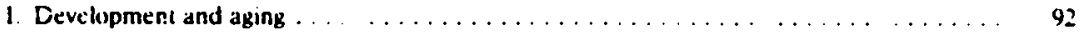

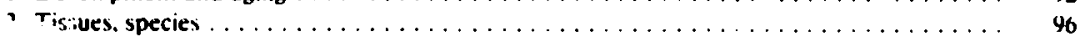

C. Experimental and pathological variation $\ldots \ldots \ldots \ldots \ldots \ldots \ldots \ldots \ldots \ldots$

1. Dietary man ,ulation. whole animal $\ldots \ldots \ldots \ldots \ldots \ldots \ldots \ldots \ldots \ldots \ldots$

i. Essen

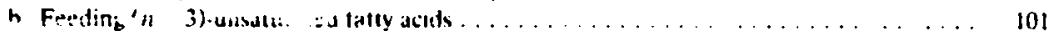

c. Feeding crucale $(22: \mathbf{l}(\boldsymbol{n}-\mathbf{9})) \ldots \ldots \ldots \ldots \ldots \ldots \ldots \ldots \ldots \ldots \ldots \ldots \ldots$

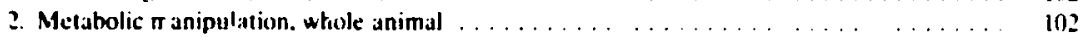

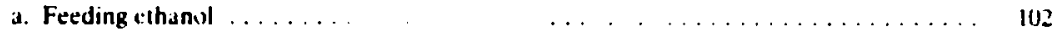

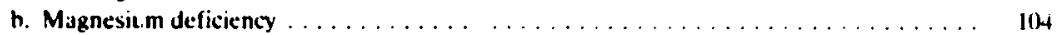

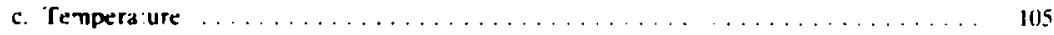

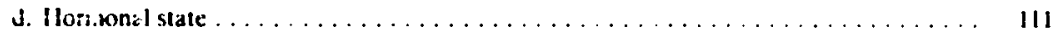

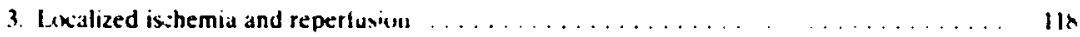

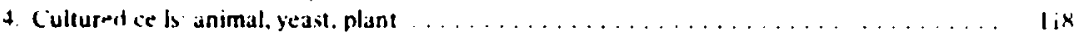

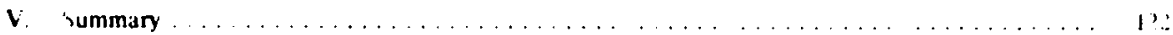

Acknouledgements $\ldots \ldots \ldots \ldots \ldots \ldots \ldots \ldots \ldots \ldots \ldots \ldots \ldots \ldots \ldots$

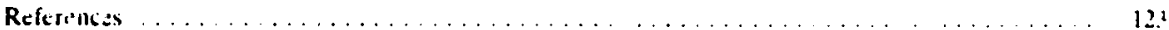

\begin{abstract}
Abbreviztions: BAI, briwn adipise tissue: BMR, basal metabolic rate (oxygen): $\tau_{1}$. llux coriaici coxefficien! (as defined anci incasuled in Ref. 228): CCC.P, eartonyl syanide in-shlorophenylhydrazcine: $C_{M^{*}}$. effective proon conductance: $\lrcorner p$. proturimotive force (in $\mathrm{mV}$.

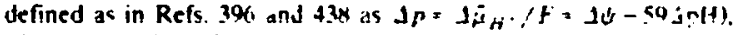
where $\mathrm{Id}_{\text {and }}$ InH are the trar.smembrane differences in elertical putential and pH: EFA. essential fatty acid(s): FCCP, tarbonyl cyanide $p$-trifluuromethuxyphenylinydrazone: $\boldsymbol{P}_{\mathrm{H}}$. aspareut proton permeability: $P G P_{H}$. 2.3-viphytanyl-sn-glycerc-1-phospho-snslycicrol-l'-phusphaic. T4, thvroxine: T3. triouknthyr. onine.
\end{abstract}

\section{Introduction}

"Almost alt the cardinlipin of the (rukaryote) ce!! is in the nner r:litoc.londrial membranes. making up atxout cone-fiftt of the cotal iipic. i: is its iritensely

Correspon Jence: F.1. Hoch. $345 ;$ Woudland Ravad. Ann Artur. MI 48ivi-\$257, USA 
hydrophobic, lipid character that gives the membrane it coherent structure, determines iss permeability. and accounts for the properties of its enzymes" [6!4).

Cardiolipins are unique to biomembranes which have coupled phosphorylation and electron-transport: bacterial nlasma membranes, chromatophores, chloroplasts, mitochondria [5(M)]. (hemiosmotic mechani ms of oxidative ,hesphorylation $[4.3 .3-4.36 .462 \mid$ most readily explain the roles of membrane phospholipids and nhospholipid-embedded protcins that carry electrons, protons and metabolites. Correlations between lipid composition and oxidative phospinorylation have been developec in a series of reviews: on lipids of subcellular particles [190], regulation of membrane enzymes [i69]. lipids of miartindrix [1.36] and chomictry and metabolism of cardiolipins $[3015,320]$.

Recent reviews on lipids and thyroid hormones [284,285] summarize evidence that thyroid hermones control genetic expression of the enzymes that synthesize and desaturate fatty acyls-CoA. The products of up- or down-regulation change mitochondrial phospho!ipid compositions and alter oxidative phosphorylation, with the result that thyroid levels relate directly with respiration in State 4 and to a lesser degree State 3. These cilanges teflect abnormal rate-temperature profilcs typical of aitered membrane lipid compositions. A special role war ascribed to the relative amounts of cardiulipins and to their very high $18: 2(n-6)$ fatty acyl centents in thyroud-sensitive tissues. Such a ciusal sequence is consistent with observations that dic: 1 y EFA-deficiency, without involving genetic mechanisms, specifically depletes ( $n$ - 6 - -unsaturated falty acyls in mitochondrial phospholipıcs, especially cardiolipins. and according to many studies increasec groton leskage under State 4 conditions.

The present review examines more wideiy the roles of cardiolipins in membranes and reeonsiders some previously reviewed dald in the light of mechanisms of proton permeability in phospholipid membranes (see section II-A), and cardinlipin-protein interactions: (section II-B). A number of conditions that ditit membrane shospholipids, some selective for cardiolipins. increas: or slow bacterial (section III) and mitockondrial (section IV) I cipiration. Bectause changes in phospholipids affect tempirature-deperdence of respiration and tra'isfe: rates of many substrate carriers. temperature and substrate are here specified together with respisutory and membrane potential da!a; sometimes tempera:ure and subitrais are crucial for cemon. strating effects.

\section{Phospholipids and membrane energy transductivn}

In the rriginal scheme of delocalized chemiormosis. the closed cyclic property of the proton circuits requires that the efflux $\left(x \rightarrow H^{-}\right) / \partial t$, to t' e outer phase in mitcochondria) through the respiratory chain $(o / r)$ equal the total relurn flux $\left(x \leftarrow x^{+}\right) / \partial t$ to the inner pleas! through the ATP-synthase (retisible ATPase) $(h / d)$, hy diftusion $(D)$ and by exchange $(X)$ acioss the membrane $[433,437]$; the balance, modified from Ref. 4.37. Eqn. 25. is:

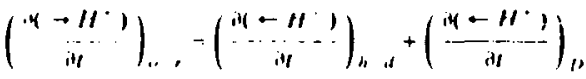

$$
\begin{aligned}
& +\left(\frac{\left.i+H^{*}\right)}{i t}\right)_{x}
\end{aligned}
$$

\section{II-A. State 4 respiration: proton lank}

Under Statc 4 conditions the potentially great proton passage through the ATP-synihase term $(h / d)$ is minimal, as shown by the failure of oligomycin to inhibit (but see IV-C.2.d). Proton reflux then dipends on the lesser conductance bu diffusion anc exchange the 'proton leak'. The 'diffusion' term implied a mechanism of passive transport of protons across a relatively ion-impermeable non-aqueous phase (the phospholipid bilayer) in the coupling membrane [437] - a phospholipid leak. (The uncoupling protein of the inner mitochondrial membrare in brown adipose issuc (BAT) is a special mechanism for an exicrnaiij regulated exchange leak under State 4 or State 3 conditions (section IV-C.2.c).) Proton reflux completely govern: State 4 respiration $[53,22 \lambda, 433,434,438,744]$. For exampie, when $\mathrm{KCN}$ is added to rat liver mitochondria to block clectr'n :iu,ipor: and a!iminate possible proton pump slippage under State 4 conditions (succinate, $P_{i}$ and viigomycin, $25^{\circ} \mathrm{C}$ ), $\Delta \psi$ dissipates with a $t_{1 / 2}$ of $1-? \mathrm{~s}$; $-\lrcorner \psi \cdot r^{-1}$ is therefore at/ributed to only passive leak ot inns [744]. Because $-\lambda \psi \cdot t^{-1}$ and State 4 respiraticn $\left(-\mathrm{JO}_{2} \cdot t^{\prime}\right.$ ) have identical rolutionships to absolute values of $\Delta \psi$ down-titrated with malonate. these workers conclude litat State 4 respiration depends on passive leak of protons.

We need to undersiand the roles of individual phospholipids in proton leak mechanisms. under Stąı 4 conditions. First. how much of the protun leak is mediated by membrane phuspholipids and how much by exchange-carrier-pioteins? Min.mization of exchange-leak: by removing ions frcm the media [466] or adding specific inhibitors of carricrs [62] does not consistently slow State 4 proton leakage $[228,673]$. Nevertheless. exchanger rycling by $\mathrm{Ca}^{2+} / \mathrm{H}^{+}[462,464]$ or $\mathrm{Ca}^{2+} / \mathrm{Na}^{+} / \mathrm{H}^{+}[68]$ is assigned a sole in mitochondrial State 4 proton circuits Hmepver, phospholipids appear to dominate in proton conductance. This conclusion is $\therefore$ ppported by similarities in permeability of intact mituchondria and protein-free menibianes. Proton permeabiiity is usually in the range $10^{-3}-10^{-4} \mathrm{~cm} \mathrm{~s}-1$ in 
mitochondria, in membranes made of their extracted phospholipids, and in some membranes furmed from stable synthetic phosphatidylcholines or phosphatidylethanolamines that usually contain two saturated f:aly acyls or one saturated and one monounsaturati:d fatty acyl [146,167,238,371.468]. Nichuls and Deamer [468] cenclute that the proton-leak via the inrier mituchondrial nembrane phospholipids car, decount for itic passive protun flux in State $\Delta$ and that it is not necessary to postulate an X-lcak.

Three mechanisms for proton conduction in phuspholipid bilayers are, in iroreasing applicability to a leak uni's State 4 conditions: (i) along the surface of anionic phospholipid headgroups, (ii) through protonophoric non-bilayer phospholipid domains anc (iii) across the hilayer.

(i) Haines [25!] nronoses that anionic headgroups of lipids in hiomembrancs conduct protons $10^{5}$-fold faster than water does, where yy protons pumped from the other side of the membrane are confined to a iurface and sonducted laterally. The cinionic polar hearigroups of $\mathrm{PGP}_{11}$, the diphytanyl ether analogues of phosphatidylglycerol phosphate (from archacbacterial membrancs, see section III). readily conduct protons lateraliy in highly condensed monolayers [522,647]. Cardiolipins are virtually the os!; anionic phuspholipid in the inner membranc of mitochondria [307j. Cardiolipins are the most acidic of eubacterial and mitoninondrial membrane phospholipids. Cardiolipin phosphoryl-groups are the most extended and rigidly fixed: the free hydroxyl zroup of the charged -phosphoryl. $\mathrm{CH}_{2}$ $\mathrm{CHOH}-\mathrm{CH}_{2}$-phosphoryl- backbone, as well as the esier carbonyi groups. contribute to the stability of an intraand intermolecular, bidimensional, hydrogen-bonded network 'hat includes water molecules of the hydration layer $[313,631]$. Cardiolipin fatty acyl saturation modulates surface conduction of protons. Escherichia coli cells that have saturated cardiolipins eject respiratorychain-generated protons from the cytosol across the membrane to the exterior; sells with cardiolipins containing mostly monounsaturated fatty acyls retain some of these pun oed protons by conducting them laterally hack to the cytosol [221] isee section lili. Sich a netwerk of asdiolipin polar grougs is thought to conduct protons lateral!y in membranes and vesicles of extracted phospholipids of $E$. roli $[516,517,648]$. chloroplasts [2:] and mitosliondria [313].

Hydrogenated beef heart cardiolipins, which contain $86 \%$ saturated fatty acyls and $9.4 \% 18: 1$ acyls. form. bilayers in aqueous dispersions and thereby conduct protons on their surfaces [313]. In contrast, natural highly unsaturated bee? heart cardiolipins form nonbilayer $H_{\text {॥l }}$ phases (see beiow), obviousiy because of different acyl packing. To conduct suriacic ....iuns, they have to be embedded in an ordered lipid bilayer. provided in mitochondria oy phosphatidylicholines anc phosphatidylethanolamines. This interplay may explain a special connection of cardiolipin unsaturation with prolon cunduction, together with a dependence on the other mitochondrial phospholipids.

However, a surface route does not readily t.spiain proton reflux in mitochondrial State 4, since net vectorial proton flux requires a route perpendicular to the membrane, and most evidence eliminates recntry through an exchanger protein. Surface routing might be important under State 3 conditions where active exchangers cotransport protons (see next sectivis?

(ii) A role as protonophores has been suggested for nonbilayer structures inside the inner mesnbrane of mitochundria that are identified through combinations of measurements by small-angle X-ray diffraction, clectron microscopy, and "P-NMR. Tlicy are depicted as an hexagona! $\mathrm{H}_{11}$ lipid phase with interior polar groups surruunding an aqueous channel, or as other nonlamellar lipid structures $[125,139,141,58$ ?]. These structures are thought to disturb the lamellar bilayers that preserve the membrane permeability bar:ier [514] and thereby vould promots transmembrane proton laakage, or to conduct protons within the membrane, which wouid shuit proiuns away from the leak.

A bilayer $\rightarrow$ heragonal $H_{\mathrm{i}}$ transition occurs rapidly and readily (low $E_{\mathrm{a}}$ ) in aqueous suspensions oi phosphatidylethanolan:ines with fatty acyls in liquid-crysta!line phase. The $H_{4:}$ torm is favored by increased fatty acyl unsaturation and carhon chain length [570], protonation of the phosphativylethanolamines and high ionic strengths. The heddgroup and acyl chair composition; of cardiolipıns modulate phase changes in model membrane systems. Neutralization of cardiolipins with cations $\left(\mathrm{Ca}^{2+}\right.$ and irig $^{2+}$ i [529] including protons, or high $[\mathrm{NaCl}]$, induces the $\boldsymbol{H}_{\|}$, thase, more readily as lice fattv acyls are changed from tetri-14:0-cardiolipin to tetr.-18:1-cardiolipin to the natural beei heart mitochordrial cardinlipins with high $18: 2$ conten, $[5: 2,1]$. Thus, if an $t_{n}$ niv : ines conduct protons intramembranally, shifts trom high polyunsaturated to high monounsaturi.ed or saturated fatty acyl iontents mignt prumote transmembraral proton flow. Nembrane proteins also affect $H_{11}$ phase formation, be! diffecinity in model svslems and intact mitochondria. Cyiuchrome $c$, which is cationic but not naturally embedded in iice mitochondrial inner membrane, specitically inc'uces the $H_{\text {II }}$ phase when liposomes contain cardiolipins pius: : nonspecific varicy of other phespholipids [138]. A car . diolipin-cytochrome $c$ complex mav facilitate dircet transfer of elecirons to ayuchrome $c$ oxidase [141]. In contrast, the presence of som. intrinsic proteins, c.b., cytochrome $c$ oxidase favors the lamellar bilayer phase. $\mathrm{Ca}^{2+}[54.3]$ or $\mathrm{NaCl}$ [514] override this p:eference and induce the $H_{11}$ pindic in an oxidase-cardiolipin syscem.

On the contrary, little if any $H_{11}$ phase exists in intact mitrechondria, and the proteins appatentis ovi- 
ride ion effects [140] Almost all the endogenous phon. pholipids in rat liver milcohondria in Statc $4(>9) x^{\prime \prime}$ :

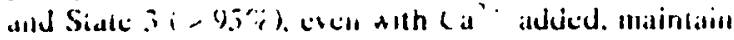
a hilayer structure over the range $\left(1-37^{\circ} \mathrm{C}\right.$. It emains conceivabile that undetected domains of $H_{11}$ phase (<2-5\% of phospholipids) are a limited route firr proton flux. Seddon et al. [582] suggen that preton. or cation-neutralized currivilipins, together with assuciated protsins might condect protons to couple electrontranspori to the ATP-synthase. i.c. in State 3. Such structures, and the su. face networks of $\mathrm{H}$-bondied cardiolipins (sce (ii) above), might be a physical balst, for postulated intramembrane coupling onc. hanisms 1:55557.714 ] (see section II-B).

(iii) Proton passage across phosphr lipid portions of nilayer membrane, hus secm.; most likcly under stalc 4 conditions. Protons do not move by classic dittusion across bilayer membranes made from pure phosphottidylcholines or phosphatidylethanolamines. Increased $\left[\mathrm{H}^{\prime}\right]$ woud acceleralc flux hy a ditfusion mechanion hat profon conductance increaises only

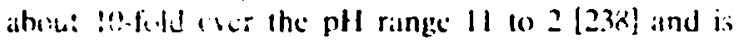
relatively constarit in liposomes over $2-. ;$ r.th units around flcutrality $[69.145,2,4-241,452.5016,5,77$. Further. sim; le affusion does not account fer protons heing about $100^{\circ}$-fold more perraciable thatn $K^{\circ}$ and $\mathrm{Na}_{\mathrm{a}}$ [14.5]. Therefore. profon-selective tran porre is reropesed to nrececel via trace amounts of natural protonophores or transient thatins o" hydrogen-bonded water molecules.

suggested protonopheres include (undelestable) traces of (i) frce fatty acids. (ii) o): superoxide anions and (iii) carbon dioxide and it: hy Irat:s.

(i) Cass al oxidatic of monufictured linid bilayers

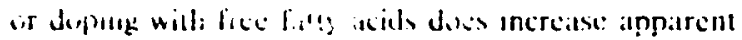
$l_{11}[6)$. and scrum allhumin (whic h strorgly hinds frice fally an ids) call lower condectanie [239.241)]. However. alhumin. dex not diminish uncoupling in mitectondria

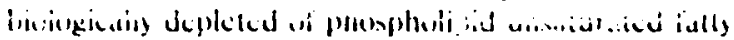
acyls. indisating that the more silturated fatty acyl chains, not frec fatty acids. conc uct the accelerated peolon leakage [?6.3] (sece section IV-C A). I:urther. fice

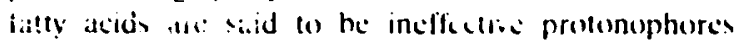

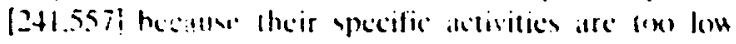

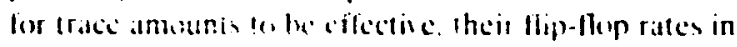
the meinbranc are tios how. they uncouple maximally at high ph where they are completely aniusic. and thici $p K$, values are much lower than resuired for transmembrane $H$-shutling. Contrariuise. the $p K$, of the cartexylic group of $18:$ ! in the polar surface of egg phouphatidy leholine multilayess is in.? which is 1,3 unit: highe: then in aqueous phase [5?]).

(ii) Carbonera and Azrone [77] propose that oneelectron reactuns in the elec ron trat.jport chaia.; reduce $(1, \rightarrow 0$; supcroxide al in whicl allacks pro-

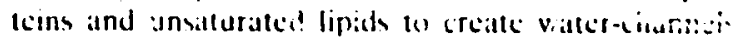

lilat increase $P_{11}$. Prolective niechanisms include conversion of supciceside radical $\rightarrow \mathrm{H}_{2} \mathrm{O}_{2}$ through superuxice dismutase action in the matrix; $\mathrm{H}_{2} \mathrm{O}_{2}$ and glutathione oxidation through glutathione peroxidase acfion completes the cycle. Formation of autoxidized protonophoric side products by mitochondrial oxidations would depend in part on fatiy acy! unsaturation. Cardiolipins are the most highly unsaturated of the mitochondrial inner membr.ne phospholipids in liver and heart. in the sense that they centain the least oroportions of saluraled fatty acyls (other pho:pholipids of ten lave greater unsaturation indices), and so have heen thought to be the mos' likely source of ricolonophoric prodicts of autsxidurions. Protective mochanisms against frec radical actions may hate special significance for cardiolinins [17.3].

(iii) Norris and Powel! [475] present evidence that. in large unilamellat vesicles of di-18: 1-phosphatidylcholine, carbon dioxide. curbonic acid ard bicarbonate could ate as proton carriers in addition to passive profun flux. $i_{13}$. is anout $10^{+} \mathrm{cm}$ s 1 at $25^{\circ} \mathrm{C}$ even when these carbonates are removed (1) be biow the deccition limit of $20 \mu \mathrm{M}$. They nots: that oxidative decarboxylaions in the milix!ondrial matrix produce carbon dioxide in quantity, question why millimolar concentrations of carbopates at the inner surface of the itener membrane de met dissipate $1 p$, and propose that $\therefore$ mechanism might involve acidic phospholipids (in

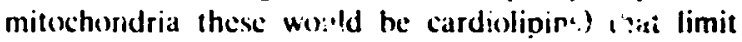
the concentratiosi. of carbonates at ih artace and retard movements of $\mathrm{HCO}_{3}$.

Piotoll permeation and water piermsatior arc interrelated in phospholipid memioranes: evidence includes the similarities in their permeability coefficients [14h] high $I$, values at temperatures abuve the phas transition temperature [167] and icmperature-depenvience [51]. Ice conducts protoms faster, through increased order. In phospholipid inemhrañes, cxicrnal water is

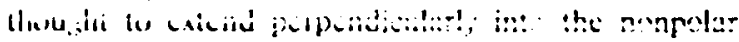
phase as a sing!e chain of watter molestles iransiently stabilized boin by hydrogen tenditig and the ordering elfect of tisc surrounding parallel (1.c... saturated) heurocarton chain $[1+6.46, i]$. Movement of protons by successive turnirg and hopping defects in the water straind can cxplain why $H^{\prime}$ cinductan. is cointiant with $J \mathrm{rH}$ hil i epralincar with $J \psi[4.52]$ and is $s_{0}$ M.idit in ter tha: that of $\mathrm{Na}^{*}$ or $\mathrm{K}^{+}$(protons can move along hydrogen tonds). Against this model are: ide implausibility of an ordering of water molecules into extended hydrogen-bonded chains in a nonpolar environment" (14.5): the absence of delectable water: and the tailure of $\mathrm{D}_{2} \mathrm{O}$ to slow conductance actoss pure phespholipid membranes although confuctance of $\mathrm{D}_{2} \mathrm{O}$ is liti-fiald less than $t_{2}, O$ in ice. Ihe watcr-chain mos.i inplics that fatty acyl saturation and increased ordi r promote promon ?ernicability. In 1 rauble's mech- 


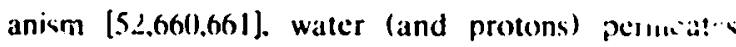
phospholipid bilayers via $\beta$-coupled configuritions ( $Z_{k 1}$-kinks) ihat move along extended saturatec acyl chains. The presence of carbon-carlon double honds which do not allow frec rotation abow: their alxi" auld block the progress of 'kink wave. Thereby, bilayers of di-18:1-phosphatidylcholine conduct prolons morc slowly than biaycers of $\mathrm{di}-18:(-)$-phosphatidyleholine [146].

Cardiolipins p!a: a special role among miicritioi drial phosphclipids in conducting nrotons. Mi xchondrial inner membrane integrity depends specifically on cardiolipins. Enzymatic digestion of cardiolipins but not phosphatidyicthanolamines or phosphatidylcholines correlates with the disruption of structurs [23]. and hypo-osmatic swelling that producus an uncouplin; proton-leak decreases cardiolipin/protein aind cardiolipin / phospholipid by $30 \mathrm{r}_{i}$, but phosphatidylethanolaminc/phospholipid by or:ly $8^{\prime} ;$ [349]

Proton perme ability in natural membranes is inverse to phosphonlipid filly ard unsalluration. sometim: in au invividu.tl phospnolipid. Some cardiolipins are not only the 'must unsaturated' of meinbranc pilospholipids he t: also the most responsive to regu!atory intluences that slter their amounts, decrease their unsaturation. and change proton leah rates (ste sections III, IV).

Interplay between properties of the apolar hydrocarbon bilayer micrion atlu the hydration and charge of ihe phospholipid surfaces deternines function of the lipid portion of membranes. Irercased unsaturation of the acyl chains augments surface binding of phospholipids to cattions [82] and hinding of cardiolipin acyl chaius to prolcins $[5,6,577.62(0)$. Cardiolipins, among the mitechondrial inner menhrane phospholipids. are not only the least saturated anc mont acidic, the mos! rigidly tixed in a $\mathbf{H}$-ronded network that orients surface water molecules and cationic residues of protecins [6.3.3]. and when neutralized are the mest favorable (1) the formation of nonbilayer, poolen-condusting struc tuics [570.582], hut they are also the mist lecalised to (1.). (the inner) face. ints concentration counters ifi part the eilution of these special pronert:... ing inc paucity of saruiviipins. Cardiolipins thereny ean collduct protons and chelatc cations. They may also act as effectors 0 . specific receptors, and orient proteins (sece seccion! H-B).

Bilayer nembrane stability depends on the molecular : ametry and 'shapes' of the ptospholipids ( / (W)i Cardiolipin headgrouns are important in determining ine shape of the molecule, and their iateractions with ions alter lidid phase behavior [515!]. The 'monola'ver

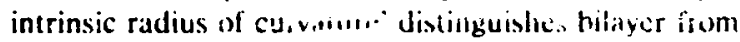
nonbilayer phospholipids [23.3]. The presence of unsaturated fatty acy! groups in membrane phospholipids

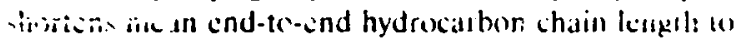

a minimum, in arder of effectiveness $18: 3(n-3)>$ If: $2(n-(n)>18: 1(n-9)$ accyls $[6(1)]$. The extended cardiolipin polar head group is associated with very high propirtions of $18: 2(n-6)$ acyls, less $18: \mid(n-9)$ acyls and few saturated fatty aryls in cardiolipins from liser. heart and kidncy. Unsaturated fatty acyls $i$; these cardiolipins would widen the hydrophobic ends to for $n$ a truncated conce, and their monolayt: pasitioning should emphasize the effects of this structurc. Conversely. cardiolipins th:t contain less polyunsaturated fatty acyls and more monounsaturated fatty acyls. and saturated fatty acyls would have closer packed hydrocarlun chains and a cylindrical or inverted cone structure. The caicended hodrophilis group of highly a ratturated cardiolipins. with its four kinked long-chain hy droptobic moictics, creates regions of smatl culvaturc in the membrane [427]: these cardiolipins find their way to the inner surface of lipesomes formed trom mixtures of phospholipids $i+f i j$. (ardiolipins containing four 16:0 fatty acvl grounc form bilaycr liponon:es 52.6]. and the mostly sat!reted catdiolinins of hast:-rid are situated in beth taces of the plasma merabrane (see section ! $i$ ). However. the llatuiall; linestusated cardiolipins of beef heart ars also on both sides of the lamellac it sonicalted vesicles [377], perhaps hecausc cardiolipin: are more than surs of the total phospholipids.

Highly unsatmated cardiolipins from mitochondria ace not usable as ine in studies of proton flux in protein-free vesicles because they do not form bilavers; they must be stludied in bilayers formed by other hh... pholipid, [31.3] (see above). Hydrogenated heef hear cardiolipins (18:(1)-cardiolipin) [31.3] and sunttectic calaislipins with four similar saturated fatty aty! groups form bilayer memor ase that have lacrmotiopic phase transitions characieristic of phospholipid bilayer assemblies [5.66.570]. Bilayer membrines of even the relativeiy saturated bacterial cardiolipin. (a.e sec in.t III-A) are also (oo unstable for nic...urements of proioii condictiance, in contrast i.) piosphatidylglycerols and phosphaidy lethanolamines [295].

Arificial memprasics iilat sontann relatively saturated phosphaucischolines + phosphatidylethanolamines leak protons as fast as tatturai merabranes that contam unsaturated phosphatidyicholine: + phenphafiuylellanolamenc.: + cardiolipins [468): if ursaturation. does not matter, the cardiolinin are ir.rt. Bui tensittu. ration astects procon movement. Prosph.lipid fatly acyl unsaturation, chain length and thermal disorder all inf? ience micmbran: prowon permedilities, but nor always the satne wil; in naturat and artificial rikmhranes. $P_{1 !}$. increases jincarty whe-: fally acyl unsatu-

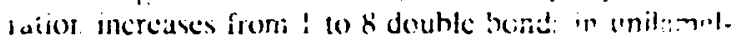
!ar vesicles of phosphat dyls: alines [5016]. Given a tixud

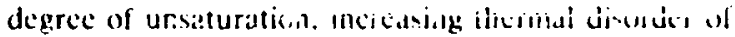
fatty acyls raises $\ddot{z}_{11}$ in ingle lanıi:ier vesicles of 
di-14:01-phospliatujate [167] and in hilayer liposromes made from horse bean phospholipids [553]. In cintrast, decreased mitochon irie! unsaturation. aimost completely confined to cardinlir:ns, accelerates proton leakage, c.g., in EFA-deicic:rcy [263.284] (sec section IV).

Perkins and Cafiso [507] suggest that fundamentally different mechanisms might operate in sonicated vesicles and planar hilayers. From the disparate effects of fatty acyl unsaturation on proton permeability and bilayer structures, mechanisms also differ between model membrane systems and mitcchondrial inner membrane bilayers.

\section{II-B. State 3 respiration: cardiolipin -protcin interactioms; oriemtation}

In contrast to the regulation of State 4 respirat ion by $\Delta p$ and the very slow reflux proton leak, the more rapid ratc of State 3 respiration is governed by $\lrcorner p$ and the balance between proton ejection and rellux [247.248,435]. The generators of $\Delta p$ operate under both State 3 and State 4 conditions. They are proteins: substrate carriers (plus the $P_{i} / H$ symporter with dicarboxylates), dehydrogenases and electron-carrier proton-ejectors. In Stat: 3 , the rapid rate of proton ejection may elicit limitation by some e!ectron-transport step(s), in contrast to the lesser demands made by the slower State 4 rate. In State 3, 1apid proton reflux Is initiated by proteins: the ADP, ATP carrier exchanges external ADP for matrix ATP, the $\mathrm{P}_{1} / \mathrm{H}^{+}$ symporter acts, and increased matrix ADP/ATP ratio and $\left[P_{i}\right]$ activate massive proton reflux through the ATP-synthase. Augmented proton current accelerates respiration 5- to 18-fold over the rate allowed by proton reflux across phospholipids under State 4 conditions, and is regulated by the relative capacities of proton ejectors and reflux pathways. The contributions of these processes have heen formulated and measured as flux control coefficients, $C_{\mathrm{i}}$, and $\mathbf{\Sigma} C_{1}=1.0$ [228].

To discern roles of cardiolipins we must know to what degree carricr-proteins or phospholipids mediate State 3 proton flux, and then consider cardiolipin iriter. actions with those protcins for regulatory properties. Proton flux under State 3 conditions stops the cytosol $\rightarrow$ matrix proton leak across the membrane phospholipids. That the leak: may stop because inner membrane ultrastructure chan zes in the State $4 \rightarrow$ State 3 transition does not seem to have been considered. Electron micrographs of isolated and in situ rat liver mitochondria show that the discrete cristae of the inner membrane condense into irregular folds [243], which should alter packing of inner face cardiolipins that normally seek concave surfaces. Two mechanisms for cessation of the leak are put forward. One observes that $\Delta p$ is high in State 4 and becomes lower in State $\therefore$ and that proton conductance across phospholipids drops exponentially with $J_{p}$ in this range [459]. This mechanism leaves the proteins to conduct protons. The other proposes that protons evolved by the electron transport chain circumverit the tranımembranal proton leak by gaining direct intramembranal access to the $F_{1}$-ATPase $[555,556]$. Little direct evidence exists that these intramembranal protons are conducted lateraliy by phospholipids, c.g., via surface headgroups of cardiolipins or their analogues (sections II-A and III). Minimal ${ }^{31} \mathrm{P}$-NMR signals exist in rat liver mitochondria in States 3,4 or $3 \cdot d$ for nonbilayer $H_{11}$-phase protonophoric lipis siiciuules that invoive cardiolipins and might also serve this function, and the phospholipids are more than $95 \%$ in bilayer structure [140]. Thus, proton passage through mituchondrial carrier proteins appears to limit State 3 respiratio:.

Mitochondrial proteins. almost all lipophilic and/or hasic [320], bind most strongly to acidic phospholipids, particularly to cardiolipins among the inner membrane phospholipids. Many hut not all are carrier proteins in oxidative phosphorylation and their contribution to State 3 regulaticin differs according to tissue and age of the eukaryote' 'sec Table I and Refs. 284, 265).

At least several of these mitochondrial proteins are genctically related. One gene for the ADP/ATP carrier is expressed in heart, another in intestine, and both of these together with a third in liver [119]: all are homologous with the carricrs of $P_{i}$, of $\alpha$-ket (oglutarate malate, and of dicarboxylates, as wel! as with the protonophoric uncoupling protein in BAT mitochondria (sec IV-C.2.c). This suggests a common evolutionary origin $[16,36,3,564]$. The primary structures of an ADP/ATP carrier, the $P_{i}$ transporter, and the uncoupling protein reveal that each has three 109-residue positively charged homologous domains: each domain comprises two homologous $\alpha$-helical stcitions separated by a more hydruphilic segment [363]. Most of the hydrophilic portion faces the matrix, where most of the cardiolipins are. All these carriers function through common mechanisms that may involve $\mathrm{H}^{+}$-anion cotransport. except for the uncoupiing protein [363].

The high affinity of cardiolipine, usually from heart mitochondria, for many basic or hydrophilic membrane proteins makes it difficult to characterize cardiclipinprotein interactions that regulate State 3 respiration. However, beet heart caruiolipins are the most effective phospholipids that modulate the secondary structure of inner membrane proteins and modify their kir "ic parameters [418]. We know that diet-induced alterations of fatty acyl compositions of membrane phospholipids change the cooperativity of several effector-enzyme systems [182]. In one case, State 3 respiration slows when rats incorporate fed linelaidic acid $(18: 2(n-$ 6)tr,tr) into heart mitochondrial phosp:atidylcholines and phosphatid. iethanolamines but not cardiolipins; 
State 4 proton leakage, like the cardiolipins, does not change [115] (sec section IV-C).

Reconstitution experiments on isolated inner $\mathrm{mem}$ brane proteins have been used to study the role of cardiolipins. These pose several problems of interpretation for State 3 regulation, as will be detailed. The criteria for reconstitution are variously the strength of cardiolipin-hinding to proteins in situ or isolated and perhaps delipidated, catalytic activity and proton-ejection activity. Cardiolipins are difficult to remove completely from some inner membrane proteins. perhaps because some cardiolipins are huried in the proteins, c.g., the cardiolipins in intact rat liver mitochondria are less accessible than phosphatidylcholines and phosphatidylethanolanines to reduction by a Pd-complex [577]. Despite the known retention of phospholipids, many purified and 'delipidated' protein complexes are reconstituted without lipid analyses. To test for nonspecific effects of phospholipids, asolectin (soybean phospholipids) vesicles are used, but asolectins contain cardiolipins as $10 \%$ [454] or 25\% [447] of total phospholipid P.

TABLE !

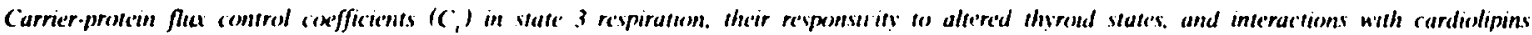
(summarised in part from Rof. 284)

Abhreviations: CI. cardiolipin: CP. carnitine palmitoyl

\begin{tabular}{|c|c|c|c|c|}
\hline \multirow{2}{*}{$\begin{array}{l}\text { Carricr-protein: } \\
\text { milochondria }\end{array}$} & \multirow[t]{2}{*}{$\sigma_{i}$} & \multicolumn{2}{|c|}{ Cl. interaction } & \multirow[t]{2}{*}{ Ref. } \\
\hline & & Binding & Activity & \\
\hline Monocasboxylate ': heart * & $(1) .37)^{a}$ & specific & + & 494 \\
\hline Dicarboxylate ${ }^{\text {th }}$ : liver ${ }^{*}$ & 11.293 & specifis & $:$ & 673 \\
\hline livet fetail * & 10.12 & & & 25 \\
\hline Tricarboxylate ': liver " & & specific & + & $\$ 95$ \\
\hline CPTransferase I ${ }^{\dagger}$ : liver ${ }^{*}$ & & specific & $-\cdot$ & see 2k4 \\
\hline CPTranslocase ${ }^{\dagger}$ : liver ${ }^{*}$ & & :pecific & $+\mathbf{J}$ & sec 284 \\
\hline Dehydrogenase ". NADH: heart * & $0.37^{\cdot 1}$ & snecific & $+d$ & 155 \\
\hline substrates": liver : & & llone & & 55 \\
\hline B-hydroxybutyrate: liver * & & specific & $-\bullet$ & 36 \\
\hline Cytochrome $c^{\prime}$ : liver ${ }^{*}$ & & non-spec & 1 & 157 \\
\hline Cylochrome $h_{1}{ }^{\prime}$ : liver * & 0.0 .3 & specific & + & $6,7.3$ \\
\hline Cylochrome $a a_{3}{ }^{\top}:$ liver & 0.17 & specific & + & 673 \\
\hline hciart & 0 & specific? & + & $513.67 x$ \\
\hline yeast & 11.55 & non-spec & + & $421.69)$ \\
\hline ADP/ATP ${ }^{\text {h }}$. liver & 11.24 & specific & + & 073 \\
\hline$(P, 3 \mathrm{mM})$ & 11.45 & & & 374.375 \\
\hline$(P, 10 \mathrm{mM})$ & $(1.19$ & & & \\
\hline liver. fetal * & 0.15 & & & 25 \\
\hline hearl & li.ti & specific & & 155.35 .5 .356 \\
\hline yeasl $\left(P_{1}, 1.5 \mathrm{mM}\right)$ & 11.2 & & & 421 \\
\hline$(P, 7.7 \mathrm{mM})$ & 0 & & & \\
\hline$P_{1}{ }^{\text {th: }}$ liver & (1) & specific & + & 597.673 \\
\hline liver. felal * & (1).1.? & specific & + & 25 \\
\hline yeast $(P,(1.5 \mathrm{mM})$ & 0 & & & $20,42 !$ \\
\hline$(P, 7.7 \mathrm{mM})$ & 0.5 & & & \\
\hline Proton leak ${ }^{\ddagger}$ yeast $(P, 0.5 \mathrm{mM})$ & 0.4 & & & 421 \\
\hline ATP-synthase ${ }^{\prime}:$ liver $(P, 10 \mathrm{mM})$ & 11 & specific & + & $47.22 x$ \\
\hline$(P, 3 \mathrm{mM})$ & 0.41 & specific & + & .374 .375 \\
\hline$(P, \mid(1) \mathrm{mM})$ & 0.17 & & & \\
\hline liver, fetal * & 0.6 & specific & + & 25 \\
\hline heart & 0.46 & non-spec & + & 155 \\
\hline$C a^{2 \cdot}:$ heart* & & specific & + & 159 \\
\hline $\mathrm{K}^{*}{ }^{t}$ : liver $*$ & & & & $\$ 99$ \\
\hline Uncoupling protein $^{\text {th: }}$ BAT * & 1.0 & & & 465 \\
\hline NAD(PXH): liver * & & non-spec & - & 566 \\
\hline
\end{tabular}

* Responds to altered thyroid state: 'generates or ${ }^{2}$ consumes $1 \rho$ [248.435):

a rat cardiac pyruvate carrier limits State 3 [596] and is included in NADII dehydrogenasc 6 , [155]:

n common evolutionary orıgin [16.564):

r makes sciivity latent:

$\checkmark$ orients:

c soluble isocitrate, glutamate, and malate dehydrogenases, and membrane-bound succinate ${ }^{c}$ and glycerol-3-phospliate dehydrogenases:

promotes $H_{11}$-phase. 
The following mitochondrial proteins and complexes, and th.eir relevance to Siate 3 regulation. will be discussed: (a) substrate carriers: (h) NADH dehydrogenase; (c) cytochrome $h c_{1}$; (d) cytochrome $c$ oxidase: (c) ATP-synthase; (f) ADF / ATP carricr: ani (g) $P$, carrier. In addition. involvement of peripheral cardiolipins in binding certain peptides and proteins especially exemplifies orienting effects.

(a) Substrate carriers. Cardiolipins arc the only phospholipid that keeps the following mitochondrial substrate transporters active during purification and reactivates them after delipidation: those for mono-, di- and tri-carboxylates, $\alpha$-ketoglutarate, aspartate/ glutamate, and the palmitoylearnitine transferase plus (acyl)arnitine translocase system (for references. see Ref. 284 and Table 1). The dicarboxylate carrier is a major regulator of State 3 iespiration $\left(C_{1}=0.33\right)$ in mitochondria from livers of adult rats and $l e s, 30$ in fetal rats. The pyruvate carrier plus the NADH dehydrogenase contribute a $C_{\mathrm{i}}=0.37$ toward icgulation in heart but not in liver mitochondria [596]. Succinate dehydroger.asc apparently contributes a $c_{1}<0.14$ in rat liver mitochondria [228].

(b) NADH dehydrogenase (and/or the pyruvate carrier) is one of the two dominant regulators of State $3\left(C_{\mathrm{i}}=0.37\right)$ in rat heart mitochondria [155] but regulates in no other mitochondria studied. Phospholipase A hydrolysis of cardiolipin, but not phosphatidylcholine or phosphatidylethanolamine. solubilizes the NADH dehydrogenase of intact mitochondria of beef heart, which implicates cardiclipin in its binding on the matrix side of the inner membrane [23]. Reconstitution of the lost activity of extensively delipidated (1).2\% phospholipids) NADH dehydrogenase (NADHubiquinone reductase from beef heart mitochond:ia) requires cardiolipin specifically, other phospho!ipids non-specifically as dispersants or orienters [199].

(c) The cytochrome $b c_{1}$ segment in delipidated complexcs is activated by several phospholipids but oniy in the presence of cardiolipins; in milochondria it is inhibited by adriamycin (which reacts with cardiolipins) (see Rei. 284). Beef heart mitochondrial ubiquinol-cytoch:ome $c$ reductase, purified and delipidated by am$\mathrm{m}$ inium sultate and cholate fractionations, retains 10\%; if the original phospholipids, which in turn are $65 \%$ cardiolipins; remova! \& \& residual phospholipids by more Urastic extractions denatures the complex [7.39]. A quinone-like inhibitor of electron transport in the undelipidated reductase iinds 10 an acyl group of a structurally essential cardiolipin molecule in the quinol oxidizing site of the $b c_{1}$ complex [732]. This cardiolipin is retained even in 'delipidated' reductase. The $b c_{1}$ segment regulates minimally in rat liver mitochondria [228] but significantly when cardiolipins are altered [673] (see section IV-C.2.d); it does not regulate in heart mitochondria [155]. The yeast mitochondrial uhiquinol-cytochrome c reductase, when delipidated like the heef heart complex o: by hexane extractions, loses almost all electron (ransport activity and its nornal 1:1 antimycin binding [66.3]. Chloroform-methanol extracts of native and hexane-delipidated complex contain similar amounts of $c$ diolipin, phosphatidylcholine and phosphaticylethanolamine; further addition of these phospholipids in $1: 1: 2$ ratios restores ' $) \%$ of activity, asolectin or cardiolipin restores $60 \%$, and cardiolipin also brings back antimycin binding.

(d) Cytochrome $c$ oxidase in mitochondria catalyzes reduction of molecular oxygen to water while it transports (pumps) one proton from matrix $\rightarrow$ cytosol for each electron transferred. Cytochrome oxidase is catalytically active when isolated from beef heart mitochondria by methods that may involve mild solvent extractions, and retains approx. 50 phospholipids including cardiolipins, presumably as an exterior shelt (see Ref. 413): however, some cardiolipins seem to be buried in the protein complex and resist such extractions (see below). These phospholipids have been more or less cleared by (i) further more vigorous extractions. which pose risk of protein denaturation, or by (ii) exchange with di-14:0-phosphatidylcholine, which preserves the proteins. Effects of added phospholipids on catalytic activity have determined specificity, but results of these two procedures have evoked different conclusions.

(i) Serial extractions : nvolving Triton $X-100$ and glycerol delipidate isolated enzync $[24,198,218,549$, 678]. Cytuchrome oxidase so stripped of all but 2-3 incl of cardiolipin per mol enzyme, and dispersed with lyso-pirosphatidylcholine, had the molecular activity of uncxtracted enzyme at $25^{\circ} \mathrm{C}$, about $160 \mathrm{~s}^{\prime} '$ [549] or $4(x)$ $\therefore$ [678]; the latter workers stressed the point that assays that allow only half-maximal activity could mask or change specific lipid requirements. Further extractions partly reduced activity and removed all but about I cardiolipin per mol: reconstitution required 2-3 beef heart cardiolipins to restore full catalytic activity [678]: phosphatidic acid reconstituted in one study [218] but not in another [198]; all other phospholipids were ineffective although they bound to the delipidated oxidase. Thus. cardiolipins appear to be specific for full catalytic activity of this cytochrome oxidase when it already contains onc molecule of unextractable cardiolipin. Cardiolipins have been described as a prosthetic group of such preparations. involved in cyclic transport (pumping?) of monovalent cations (although $\mathrm{H}^{+}$is omitted from the list) [197]. Vik et al. [678] concluded that the cardiolipins bind the substrate molecule cytochrome $c$ at the low-affinity site on the oxidase through electrostatic interaction of their anionic groups with several cationic groups around the heme edge, which activates by orienting the two molecules for electron transfer. Beef heart cardiolipins with the natu- 
ral 92\% $18: 2+8 \% ; 18: 1$ acyl content :r with sinstituted 47\% 18:2 + 52\%; 18: 1 acyls activate squally offectively, cardiolipin substitutred with like; $6: 0$ acyls $i:$ half as effective. and removal of two of the four ratural acyls abolishes catalytic activation [1.31].

(ii) Substitution with di-14:01-phosphatidy!choline produces'd beef heart cytochrome oxidase that contains 0.19-0.06 mol cardiolipin per mol and has the activity of the original enzyme, approx. 160 $s^{-1}$ [2]: cardiolipins are not essential for catalysis. By EPR measurements of competitive displacement of bound fatty acyl spin-labeled phosphatidylcholines and cardiolipin. Cardiolipins are the most highly specific for about 50 cnzyme sites; presumably cardiolipin polar sites interact with lysyl residucs at the polar-apolatr interfaces on subunits III. V, VI and VII [413.512.513]. and threc or four acyl chains are neccssary for optimal binding [513]. Activation of lipid-substituted oxidase was thought to facilitate interconversion between two oxidase conformations [2].

Purified cytochrome oxidase from beef heart, when reconstituted into phospholipid ve:icles, also pumps protons while transferring electrons $\left(\mathrm{H}^{*} / \mathrm{c}^{-}\right.$ratios are 0.5-1.0) and changing conformation (see Refs. 84, 4117. 617). Modifications of the enzyme, among them depiction of subunit III that is the ' $\mathrm{H}^{+}$-channel' and may bind cardiolipins (see above), lower $\mathrm{H}^{+} / \mathrm{e}^{-}$stoichiometry without much change in catalytic rate (uncouple: sec Refs. 392, 617), which is ca. 2(1) s $\mathrm{s}^{\prime}$ at $25^{\circ} \mathrm{C}$ [469]. The lipid contents of the enzyme preparations, some of them detergent-treated, used in the cited studies have not been documented. (Symmetrically, the above cited studies on the certifiedly lipid-depleted and lipid-reconstituted oxidase present no data on proton-pumping; some may be uncoupliad and so incompletely icconstituted'.) presumably, the purified enzyme retains at least the most firmly bound cardiolipins. In addition. the reconstituting vesicles are asolectins which naturally contain cardiolipin (see above). A possible role for headgroups of a $\mathrm{H}$-bunded network or $\mathrm{H}_{11}$-phase of cardiolipins, starting in the ligand-binding $\mathrm{Cu}$ center of the membranc-embedded oxidase, is suggested by a diagram of P. Mitchell [436] that depicts a 'proton conductor' to the outer aqueous domain.

The apparent disagreements on the requirement for cardiolipins for beef heart cytochrome oxidase activity may be academic with regard to State 3 regulation, in two senses. First, the finding that di-14:()-phosphatidylcholine provides a low level of activity indicates that cardiolipins are not chemically specific for oxidase catalysis, but since the oxidase in the inner membrane sees no di-14:0-phosphatidylcholine, and phosphatidylcholines and phosphatidyiethanolamines do not reconstitute partly delipidated enzyme, the cardiolipins seem to be 'biologically specific'. Second, th.e notion that this oxidase may be 'the major regulatory compo- nent of the clectron-transport system [2] cites Owen and Wilson [487]. who assumed that kinctic control generally operates only at the reduced cytochrome $a_{3}$ interaction with oxygen because ail proximal electron flow ratc stcps are very rapid in compaison. In rat heart nitechondria the cytochrome oxidase $C$, cannot be $>0.17$ since measured $C$, values (pyruvate, malate, $25^{\circ} \mathrm{C}$ ) of the ATP-synthase plus the NADH dehydrogerase (and pyruvatc carricr) amount to 0.83 [155]. In rat liver mitochondria $C_{\text {, }}$ is minimal $[47,20,3]$ or equal to $0.17[228,673]$ but ailered cardiolipin amounts and

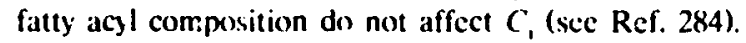

Yeast cylochrome oxidase basal catalytic activity depends on the binding of 9 mol of cardiolipin per mol enzyme that resist exchange witi jeteigerli, dric can ha enhanced by either phosphatidylcholine or phosphatidylethanolamine [679]. Activity of purified yeast $a a_{3}$ does not absolutely require cardiolipins and can use substituted di-14:0-phosphatidylcholine, but these results accomodated : specific role for membrane cardiolipins in orienting cytochrome $a a_{3}$ for clectron transfer [69(1)]. In Saccharomyce's cerctisiuc mitochondria respiring in Statc 3 , cytocitiome $a a_{3} C_{1}=0.5-0.6$ (Table 1). Yeast mitochonu'ial cardiolipins (unlike heart cardiolipins) contain $96 \%$ monounsaturated fatty acyls and no $18: 2$ acyls (see section IV-C.4), which may impart to cytochrome oxidase a sate or an orientation that limits $\mathrm{O}_{2}$ reduction rate.

(c) ATP-synthase catalysis (ATPase) is cardiolipindependent. Peroxidation of rat liver mitochondrial lipids removes unsaturated fatty acyls from all phospholipids, but only the disappearance of cardiolipin 18:2 acyls correlaics with loss of ATPasc activity [395.572]. Ernster et al. [174] suggest that the cmbedment of the protonoplotic $F_{1}-A T P a s e$ in rardiolipins is necessary for ATPase function. A highly purifict. active, oligomycin-sensitive ATPasc contains two molecules of cardiolipin and less phosphatidylcholine and phosphatidylethanolamine [16.3]. A delipidated preparation of beef heart mitochondrial ATPase is best activated and made oligomycin-sensitive hy cardiolipins among the naturally available phospholipids, which was attributed to effective orientation of $F_{1} F_{0}$ and the oligomycin binding sitc [129]. From reconstitution experiments with liposomes, phospholipids are not just a residential matrix but also mudify ATPase catalytic properties [130]. Oligomycin-sensitive ATPase $V_{\operatorname{mix}}$ increases linearly with the negative charge on individual phospholipids, except for beef heart cardiolipins that activate most effectively and supralinearly perhaps because they are highly unsaturated and the other phospholipids were not. Reconstitution of beef heart mitochondrial $F_{n}$ in cardiolipin-containing asolectii; vesicles requires a protein component, $F_{0} 1$, for proper a!ignment of $F_{11}$ for proton-translocation and binding to the F, catalytic complex [237]; endogenous phospholipids 
do not appear to have been measured. ATP-:iynthases are the major regulators of State 3 in artuii and fetal rat heart mitochondria (Table I).

(f) The ADP / ATP cantier of rat liver mitochendria loses activity but not capacity to bind adenine nucleotides when cardiolipins or phosphatidylethanolamines are partly removed by sel-etive phospholipases A [625]. A complex of protcin-cardiolipin-phosphatidylethanolamine is proposed as the nucleotide translocator. Th: c carricr is a major regulator of State 3 respiration: in isolated rat-hepatocytes $C_{i}=0.26$ [161], and in liver mitochondria $C_{1}=0.29$ and changes concomitantly with cardiolipin amounts and fatty acyls (see Ref. 284). The beef heart mitochondrial ADP/ATP carrier is activated by cardiolipins, phosphatidylcholine and phosphatidylethanolamine equaily [370]; it binds all these phospholipids [158], cardiolipins the mosi strongly. Dimeric carrier isolated from these mitochondria binds six molecules of cardiolipins (with fatty acyl cormpositions similar to total mitochondrial cardiolipins) of whirh only two exchange with spirt-labeled cardiolipins $[37,303,575]$. From studies wi binding, cardiolipın fatty acyl groups need not be unsaturated to interact strongly witl, this ADP / ATP carrier when it is already bound to di-14:(1)- or di-16:0-phosphatidylcholines, but only the natural highly unsaturated beef heart cardiolipins produce significant cardiolipin-carrier aggregation. From these binding studies, the four unexchangeable cardiolipins were proposed to participate in nucleotide transport function of the carrier [303] but transport was not measured. Rat anci rabbit heart mitochondrial ADP/ATP curriers contribute a $C_{i}=0$ for regulation of full State 3 respiration $[155,355,356]$ (Table 1$)$ althougn $C_{1}$ for the rabbit carrier (succinate, $37^{\circ} \mathrm{C}$ ) is about 0.7 when respiration is partly inhibited $[355,356]$.

(g) $P_{i}$ transporter protein purified from heart mitochondria and reconstituted in asolectin vesicles is inhibited when adriamycin hinds the cardiolipins [447]. The transporter loses activity when cardiolipins are removed, and phosphatidylcholine-vesicles do not reconstitute unless some cardiolipins are added (see Ref.

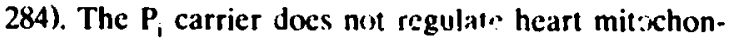
drial State 3 respiration, bist ir veast mitochondria a significant $C_{\mathrm{i}}$ diminishes with $\mathrm{JpH}$ [30].

In summary, it appears by deductions from reconstitution experiments on isolated proteins from the inner membrane that cardiolipins play a constitutive role in mitochondrial State 3 respiration. Cardiolipins bind and activate just about all inner membrane regulatory and nonregulatory (State 3) protein complexes. Fatty acyl composition affects both binding and activation. Cardiolipin reconstitution of one complex or protein applies directly to State 3 rate only if that complex regulates. But specific inhibitor titrations of these single steps in intact mitochondria, interpreted through the concepts of metabolic control analy'sis [228,673], show that State 3 respiraicon is regulated at a higher level of organization where two or more protein-mediated processes contribute acurding to :hei:" rates relative to each other. Cardiulipins may participate at this more organized level. Several reconsiiiuiion and binding experiments suggest that candiolipins orient protcins for optimal transport of clectrons (ubiquinol $\rightarrow$ cytorhrome $r$, cytochrome $s \rightarrow a a_{3}$ ) or metabolites, and align $F_{11} F_{1}$ for proton passage.

From $10 \% 1025 \%$ of cardiolipins are in the outer layer of mitochondial inner membranes and face the intermembranal space and the outer membrane in rat liver, bovine heart and pig heart [261] (see Ref. 136). In apparent disagreement, $23 \%$ of total cardiolipins of rat liver mitochondria are isolated as a 'true component' of the rat liver mituchondrial outcr membrane i307]. However, the enzymes and especially the lipids of the outer membrane so resemble those of the endoplasmic reticulum that the riembranes have been thought to be one [172]. Reconciling these views are studies on the contact sites between the iiner membrane outer face and tlic outer membrane seen in electron micrographs of State 3 mitochondria by Hackenbrock [243]. Contact sites isolated from noouse liver mitochondria as microdomains are greatly enriched in cardiolipins (27\% of phospholipids) [19] - it seems moot to assign these shared 'peripheral' cardiolipins to one surface. These cardiolipins clearly orient some proteins secondarily involved in oxidative phosphorylation. Several properties of contact sites pertain to State $\mathbf{3}$ conditions and protein orientation. (i) Contact sites exist mostly under State 3 conditions; peripheral cardiolipins act as specific receptors for (ii) selected phosphotransterases and (iii) leader peptides of import proteins, and (iv) bind liver mitochondrial carnitine palmitoyltransferase.

(i) Contact sites, as estimated from electron micrographs of freeze-fractured mitochondria of rat livers, exist transiently and depend on energy state $[57,243$, 365]. Their number is minima! when mitochondria are fixed in State 3u (uncoupled); the ratios in mitochiondria in State $3 u$ : State $4:$ State 3 are, respectively, $1: 6: 23$ or $1: 3.3: 8$ [3]. If the cardiolipins aggregated in the sites are recruited transmembran. lly from the inner face, the shift in sidedness might be involved in the loss of the proton leak under State 3 conditions, given a role of cardiolipins in State 4 proton leakage (section II-A).

(ii) Contact sites under State 3 conditions specifically bind and orient hexokinase, nucleotide diphosphate kinase and creatine kinase, but not adenylate kinase [57,365]. Thereby, hexokinase reacts more readily with ATP evolved from the ADP/ATP carrier on the inner membrane periphery than with added ATP. Outer cardiolipins are cited as a specific receptor in rat heart for the mitochondrial isoenzyme of creatine 
phosphokinase, similarly aligning access to this cariier [448]. Mitochondrial creatine kinase concentrates at contact sites [3]. The blocking effects of adriamycin, specific for cardiolipins, show that this kinase binds $(0$ cardiolipins of intact mitochondria of rat heart or liver. and to (beef heart) cardiolipin in liposomes containing other phospholipids [448]. The kinase from rat heart binds to beef heart liposome sut taces [1(0)]. Mitochondrial creatine kinase isoenzymes bind preferentially to monolayers of cardiolipin but also to other anionic phospholipids as well as to phospholipid extracts from either inner or outer membranes of mitochondria [552].

(iii) Peripheral cardiolipins interact with signal peptides of precursors of proteins synthesized in the endoplasmic reticulum and destined for mitochondria. These cardiolipins seem strategically concentrated for import through the contact sites. In vesicles prepared from phosphatidylcholines or phosphatidylethanolamines plus a cardiolipin, the two or three positively charged amino acid residues in the $\mathrm{N}$-terminal portion of the peptide bind specificallij at ite ouici sirface cardiolipin -phoṣnlioryl-glycerol-phosphoryl- headgroup [486]. The degree of saturation of the fatty acyl of the cardiolipin does not affect binding. Binding to cardiolipins as well as to surface protein groups is thought to mediate the targeting of the precursor protein complex to initochondria; the translocation of the precursor across the inner membrane requires unfolding of the precursor protein [674] which also seems to involve binding to the cardiolipin [171]. Introduction of newly synthesized mito hondrial proteins that are active in oxidative phosphorylation should increase only State 3 respiration, which depends on electron-chain protonpumping, rather than State 4 respiration which depends only on the proton-leak.

(iv) The balance between the active-form outersurface carnitine pa!mitovltransierase and the latentform inner-surface carnitine palmitoyltransferase regulates $\boldsymbol{\beta}$-oxidation in mammalian mitochondria; outerface cardiolipins may orient and activate carnitine palmitoyltransferase as well as the translocase in rat liver mitochondria, to a degree regulated by thyroid hormone levels (see Ref. 284). Recent reconstitution experiments on the transferase reveal a detergent-soluble 'inner membrane material'. most active in fasted animals, that is involved in binding and sensitivity to inhibition by malonyl-CoA, perhaps through orientation of catalytic and malonyl-CoA-binding subunits [208].

Evidence for the existence of an H-bonded network of cardiolipins in the inner face of the inner membrane [261] (see Ref. 136) is presented elsewhere in this review (e.g., sections II-A, IV). Since the anionic headgroups are all alike, these cardiolipin fatty acyl groups must be presumed to interact more specifically with lipophilic portions of cationic proteins in the mem- brane. Cardiolipin fatty acyls contain considerable information. The three significant diacylglycerol molecular species in beef heart cardiolipins are 79\% 18:2$18: 2,11 \%, 18: 2-18: 1$ and $6 \% 18: 2-18: 3:$ yeast cardiolipins have a more even distribution ef eight $16-\ddot{C}$ and 18-C saturated and unchounsaturaled acyls [575]. Rat liver heart aivi kinnev cardiolipins include at least eight molecular specics that alter proportions in response to a fat-free dist $[718,719,721]$. Cardivilipirs were first charactericed through their antigenic properties, implying their specific binding to protein. But the cardiolipin network is not fixed by covalent honds, and no known mechanism can be evoked that would form a coded cardiolipin sequence as an orienting template for oxidative prosphrylation proteins. Thus, the infor. mation for sequential reactions must lie in the protein structures, and intervening cardiolipins appear to orient at least some protein-protein interactions.

Reconstitution experiments on model systems require complerientary approaches to evaluate structural rules of riembrane lipius [535]. Reconstitutions of oxidative phosphorylation, usirig criteria of both catalysis and vectorial proton passage, and severai protein complexes integrated with various cardiolipins (inc!uding cardiolipins with fatty acyl profiles altered in vivo), complemenied with measurements of $C_{i}$ values, would be a (challenging) start in deducing possible rigulatory roles of cardiolipins in State 3 respiration from synthetic expcriments. Until such systemaiic experiments test possible regulatory effects of altered cardiolipin amounts and fatty acyl compositions, the rest of his review attempts to recognize, by induction from correlated changes in biomembrane function, regulatory roles of cardiolipins altered in situ under biological influences.

\section{Cordiolipins in prokaryotes}

Two prokaryote kingdoms, archaebacteria and cubacteria, are distinguished by different rRNA and protcin sequences [715]. Although the archaebacteria live under apparently primitive hiotic environments at very low [oxygen], high [ $\mathrm{H}^{+}$] or [salt] or [methanc], and high temperature, they are more closely related to the eukaryotes (the third kingdom) than to the eubacteria, which appear to be the most primitive (sec Ref. 511).

Membrane ether-lipids are chemical markers for archacbacteria. All contain branched-chain isoprenoid alcohols (e.g., $C_{21}$ phytanyls) as diethers of 2,3-di-O$s n$-glycerol or a more complex branched nonitol $[143,144,379]$. Biphtanyl-glycerol is tie cell membrane lipid of Methanopyrus, which grows optimally at $110^{\circ} \mathrm{C}$ [312]. Some of the diethers in methanogens and thermophiles are joined covalently at the hydrocarbon ends to form glycerol tetraethers. The glycerol-OH on one end is substituted with carbohydrate, on the opposite 
end with phospizate. in some thermorhilic archatehacteria. This molecule, with carbohydrates facing outward. is thought $t 0$ form the lipid phase of a unilamellar membrane, rather like bilayer membranes but with the median hydrophobic space fused. These nembranes are rigid (archacbacteria have wo peptidoglycan cell wall) and provide a temperatiere-stable barrice against a grattient of 4 to $5 \mathrm{pH}$ units that drives the diffusion of protons into the cell. The archacbacterium Thermoplasma acidophila, which grows optimally at $59^{\circ} \mathrm{C}$ and $\mathrm{pH} 2$ but not at all above $\mathrm{pH} 4$. maintains an internal pH between 6.4 and 6.9; $\rfloor \psi$ is about $120 \mathrm{mV}$ (positive inside) and $J \mathrm{pH}$ is 29$) \mathrm{mV}$, so that $J p$ is approx. 170) $\mathrm{mV}$ [3(09]. Neither protonophores nor inhibitors of electron transport change $J_{\psi} \psi$ or infernal $\mathrm{pH}$ : increase of exterrial pii corrciates iineariy with decreasc in $J w$ : acidophiles need no proton pump to disequilibrate protons across their membranes [489)]. Hsung and Haug [309] conclude that both $J d$ and joll are mantained passively. The proton influx that drives ATP synthesis appears to come from the low external pH and its partial negation by $\$ \psi$. which is thought to be a Donnan potential generated by charged macro. molecules that do not permeate the cell membrane.

Both energy-transducing membranes in extremely halophilic archacbacteria contain diphytanyl ether-linked phospholipidis and glyeolipids. $\mathbf{P C P}_{\|}$(2,3-diphytany!-onl-glycero-1-phospho-sn-glyccrol-1'-phosphate: $k_{1}=$ phytanyl chain) is $65 \%$ of the total polar lipid of Haichackerium: "utirubrum: it is the diphytanyl ether analogue of phosphatidylglycerol phosiphate [522.647].

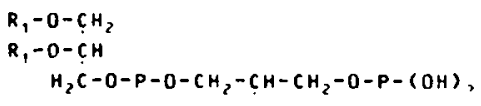

$\mathrm{OH}$

PGP 1 , its dephosphorylated products and the halophile glycolipids form bilayers in aqueous dispersions that are in liquid crystalline state from $-30^{\circ} \mathrm{C}$ to $80^{\circ} \mathrm{C}$. Their possible roic in a membrane-surface hydrogenbonded network that conducts protons laterally is discussed in section II-A.

The archachacterium Thermoplasma acidophilum grows optimally at $56^{\circ} \mathrm{C}, \mathrm{pH} 2$, and adapts its membrane lipids for growth at $37^{\circ} \mathrm{C}$ by increasing their fluidity [7.33]. The main lipios are two reperitively methyl-branched, saturated $C_{+11}$ side-chains, ether-linked to two glycerol molevules; serine and phosphate groups on some of the glycerol-OH sites provide acid polar groups. The shift from growtin at $56^{\circ} \mathrm{C} \rightarrow 37^{\circ} \mathrm{C}$ diminishes lipid phosphorus by $10 \%$, halves serire moicties and doubles acyl cyclization. The resultant increased membrane fluidity alters membrane-dependency (Arrhenius profile) of ATPase activity: activities are lower over the temperature range of measurements and transition temperatures decrease by $7.5^{\circ} \mathrm{C}$.
III-A. Cardiolipin / phospholipid ratios and faty acyl composition

The cubacteria and the cukatyotes hranch faitly early from the archacbacteri.: in a universal phylogenetic tree determined from comparisons of I.2S-rRNA sceucnees [715]. The phospholipids of eubacteria prokaryotes are fatty acyl esters of 1.2-st-glecerol-3phosphate. like those in cukaryotes [217]. Cardicispins (diphosphatidylglycerol: $R_{2}(O O)=$ fatty acyl ester) resemble a portion of PGP 11 :

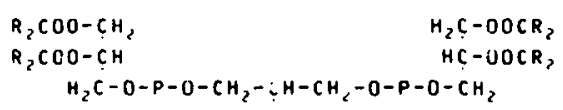

$\mathrm{OH}$

Cardiolipins in cubacteria are synthesized fomin :We molecule, of phoxphatidylglycerol [188,305]. Phosphatidylglycerols compise 10-35\% of the total phospholipid phosphorus and are tha maijor nhoopholipid $(501-80 \%$ of $P)$ in sime eubacteria [3115].

As shown in Table Il. cardiolipin per total phospholipid conicnts are up to $15 \%$ in $M$. lysodcikicus. $S$. aure"us. Salmonella typhimurium. B. cerens, up to $40 \%$ \% in the Actinomycetes [346], and 80\% in a form of $s$. aurcus [305]. Fatty acyl compositions of eubacteria vary widely (Table II). Some cubacteria thereby maintain plasma membranes in environments as extreme as those resisted by the lipids of archacbacteria. The iatty acyl composition of cardiolipins and phosphatidylglycerols is usally similar. and in prokaryotes sometimes differs from that of other phospholipids [217].

Cardiolipins and phosphatidylglycerols. as well as other phospholipids and phosphoglycolipids, are almost equally distributed in the inner and ouler faces 12:3. respectively) while the gucosylglycerides are completely outside in $A$. Koillawii membranes [554]. Cardiolipıns are cqt dily in and out, phosphatidylglycerols all outside, phosphaidylinositols all inside in M. lei.sodeikticus. From the similar polar backbone giycersol groups of cardiolipins and $\mathbf{P G P}$, it seems that cither could participatc in lateral conduction of protons via $\mathbf{H}$ bonded networks. The backbone glycerol in bilayer membranes made from cardiolipins purified from $E$. coli in stationary-phase, $37^{\circ} \mathrm{C}$. is more rigid than other phospholipid headgroups and remains so in membranes that contain $20 \%$ cardiolipins and $80 \%$ phos. phatidylcholines [8]. The corsesponding glycerol in phosphatidylglycerols has free motion: the conversion of phosphatidylglycerols $\rightarrow$ cardiolipins would increase order in membrane surface, and even minor amounts of curdiolipins could contribute to proton novements.

Both fatty acyl composition and relative proportions of bacterial cardiolipins (usually uniquely or more markedly among the phospholipids present) vary with, and respond to, growth stage, presence of oxygen, 
temperature. illumination nroton refux. The changes induced suggest that cardiolipin paricipate in enerey transdustion.

Growth phase: the growth pilase of hateterial cells affects cardiolipin / phospholipid ratios. cardiolipin and phospholipid fatty acyl comessitioni alld membrane funition. In cxponemitial growth phatse at a given temperature. cancifolipins of $l:$ coli contain 70$)^{2} 18: 11 n-$ 7) (cis-vacceroyl) acyl groups (Table II); unsaturated fatty acyl contents of cardiolipin. a phesphat dyytglycerols - phosphatidylethanolamines $[36(1)]$. When exponential shifts to stationary phase, $18: 1$ acyl content in

\section{TABLI: II}

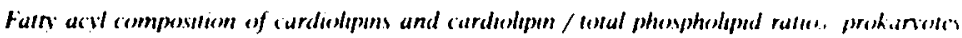

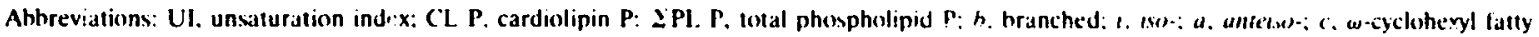
acyl: cy. cyclopropane methylenc component: 1 . Iuberculosicaric acyl (I0-methyl-14: (1). V'ariaus unspecified unsaturated fatty acyl groups ucre omitled in these studies hecause they were minur components.

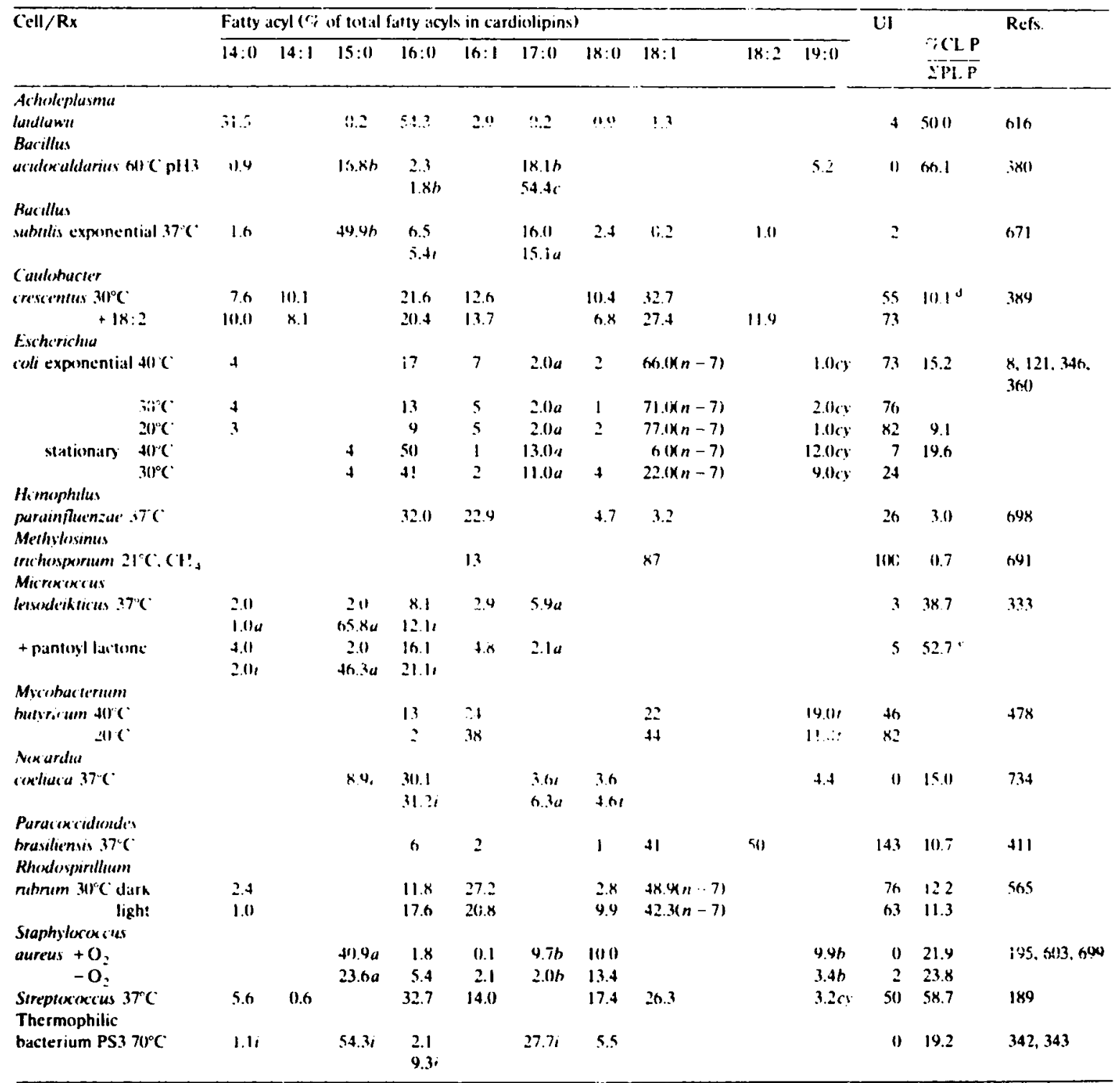

diphosphatidylglyc:rol/SPL = 78\%:

c byso- $\mathrm{CL}=20.4 C^{\circ}$ in addition. 
cardiolipins decreases strikingly to as low as for. while saturated fatty acyls content (expecially $1 \mathrm{~h}:()$ but also) nonlir ear 17:0) atd 19:0 acyls tise io si\%;. cardiclipin/ shospholipid increases and relative amounts of phosphatidylglycerol, the cardiolipin parent compound. decrease stoichiometrically. Analogously. Thiobacilles thiooxicans growing at $30^{\circ} \mathrm{C}$ increases cardiolipin/ filospholipid proportions lincarly from $7 \% ;$ (day 11 to $21 \%$ (day 8) whilst phosphatidylglycerol/phospholipid decreases [6(0)1]. In $E$. coli, at the same time cardiclipins increase, an enzyme methylates $16: 1$ acyls across the unsaturated bond to form cyclopropanc-19:0, a saturated fatty acyl [123]. In the exponential $\rightarrow$ stationary phase transition, the fatty acyl compositions of phosphatidylglycerols aind phosphatidylethano:amines change but little: the fatty acyl contents of cardiolipins are the most sensitive to growth phase.

Growth phase mav affect analytical recovery of cardiolipins. In stationary growth phase, routine chloroform-methanol extractions recover as little as $5 \%$ of the cardiolipins and all the phosphatidylglycerols and phosphatidylethanolamines from whole cells of several Gıam-positive bacteria, but in carly exponential phasc all cardichupins are extracted [i:i7]. Carjiolipin/ phospholipid ratios are up to $57 \%$ when the iniuciupeptide-teichoic acid components of the thick cell walls of stationary phase cells are removed, e.g.. by lysozyme; exponential phase cells have thinner walls that leave cardiolipins accessible. It is not clear if the altercu fatty acyl compositions of the cardiolipins in the different growth phases contribute to cardiolipin-cell wail association.

Electron-transport rate increases with growth rate, as exemplified by Klebsiella acrogenes [456]. In carly. exponential stages of growth, microbial cells oxidize carbon substrates efficiently, to transport solutes and synthesize biomass. When growth stops, cells are thoughi to oxidize less efficiently by uncoupling oxidittive phosphorylation or turning ATP over rapidly in a futile metabolic cycle [271.649]. Uncoupling mechanisms in the membrane (see Fqn. 1) include decrease: in overall $\mathrm{H}^{+} / \mathrm{e}^{-}$ratios (e.g., through climination of proton-translocating loops in electron-transport chains [338]), and dissipation of $J_{p}$ by reflux of extruded protons through a phospholipid-leak or an $F_{1}$-leak. One might add that conversion of State $3 \rightarrow$ State 4 respiration, when diminished ATP-utilization ciepresses [ADP] and stops phosphorylation, increases $J p$ and does not decrease efficiency of proton cotransport through exchangers. Intact bacterial cells show no respiratory control when ADP $+P_{\text {i }}$ are added (see Ref. 200), nor should they since bacterial plasma membranes have no (and need no) ADP / ATP carrier [331]. State 3 and State 4 arc distinct in everted particles from bacterial membranes (see below).

An $\mathrm{O}_{2}$-pulse given to anaerobic $E$. coli cells grown at $37^{\circ} \mathrm{C}$ produces a $2-10$ ? . fold greater proton efflux in stationan-phase cells than in exponential-phase cells [221]: exponential-phase cells with cardiolipins contioining high monounsaturated fatty acyls secm to retain protons; stationary cells with cardiolipins repleted in s:turated fatty acyls lose protons (sec section II-A). In sia ionary cells the (1.-pulse is followed by lusty respiration but proton sfiluy :.: nuts:h slower [221]. apparently ret?ectung a secondary slow 'leak' of proions that equilibrates external $\left[\mathrm{H}^{+}\right]$with protons in or on the outer aspecl of the membrane. The rest of the protons extruded by electron-transport do not appear to leave the cell membrane but to be laterally conducted. to return to the cytosol via proton-driven exchange transport or ATP-synthase.

Apparent proton conductarice across bacterial mem. branes has heen mesaured by $\mathrm{JpH} / \mathrm{tunl}$ after an $\mathrm{H}^{*}$. pulise under anacrobic conditions [4(18.4199]. With $J_{p}$ assumed to the 20k, $\mathrm{mV}$ and protein contents distributed as per Neidhart [45.5]. $C_{: 11}$. in $E$. coll cells can be calculated tw be $0.08 \mathrm{ng}$ ion $\mathrm{H}^{*}$ min ${ }^{\prime}$ (mg total protein) ${ }^{1} \mathrm{mV}$ ': if membrane protein is $15 \%$ of total protcin, $C_{\mathrm{M}^{\prime \prime}}$ ' is $11.5 \mathrm{ng}$ ion $\mathrm{H}^{+}$min ${ }^{1} \mathrm{mg}{ }^{\prime} \mathrm{mV}$ ', a value in the range of mitochondrial conductances [462.464]. $C^{\prime \prime}$ ' is $11.7 \mathrm{ng}$ ion $\mathrm{H}^{*}$ min ${ }^{\prime}$ (me me mbrine protein) ${ }^{\prime} \mathrm{mV}-1$ under similar assumptions in Streptococcus lactss in ear'y stationary phase.

Real proton conductarices must be compared at the same $J_{p}$ because proton current has a supralincar and shifting relationship with high values of $J \boldsymbol{p}$, as pointed out by Brand and coworkers (section IV-C.2.d). Membranc ion conductance adapts to growth rate in phosoheterotrophic cultures of Rhodobacter capsulatus, an a-purple cubacterium, grown at $30 \mathrm{C} \mathrm{C}, \mathrm{pH} 7[645,646]$. Diss.pative ion current (clearly protons) increas's disproportionally and non-linearly with $\lrcorner \psi$. As growth is limited by [carbon source] or by decreased illuminaticii, conductance incredses: current/J $\psi$ is greater when substrate limitation is more scvere. Current is greater in stationary than exponential cells for similar values of $\perp \psi$ above threshold. Most of the current at maximal $J_{\psi}$ is vid the ATP-synthase. i.c. State 3 respiration. When the $F_{10}$-channel in intact cells is blocked by venturicidin. rapid proton flux persists: the high $\rfloor \psi$ augments another conducting pathway(s). A proton-leak (perhaps similar to that in State 4) appears, as it does in mitochondrial vesicles stripped of $F_{1}$ when $F_{11}$ is blocked by oligomycin. 'Taylor and Jackson $[645,646]$ dismiss dielectric breakdown as an explanation for a new proton leak because $J \psi$ is still at physiological levels, and the phenomenon is reversible, like the cycle State $4 \rightarrow 3 \rightarrow 4$. These workers propose that a membrane gated-protonophore has a threshold at slightly greater values of $d \psi$ than does the $F_{11}$, and that decreased growth rate (somehow) depresses this threshold. Stationary-phase cell membranes, cumpared 
with log-phas: leak more protons: their membrancs have a greater preportion of cardiolipins and those cardiolipins are the most markedly depleted in unsaturated fatty acyls. As will be seen. fatty acyl composition of cardiolipins is analogously connected with proton leakage in many energy transdicing membranes

Temperature: membrane fatty acyi unsaturations vary inversely with environmental temperatures in prokaryotes and the simpler eukaryutes, which maintains membrane lipids above their melting points and retains appropriate membrane barrier and matrix properties [267,268,399,527]. Eubacteria in exponential-phase adant to decreased temperature.s hy iricreasing unsaturated fally aryls contents in memhrane phospholipids $[122,123,142,2(1), 4 \times 11]$, either without cinanging the proportions of phospholipid-subclasses or changing them slightly in $E$. coli so that cardiolipin/ phospholipid ratio halves, phosphatidylglycesol/ phospholipid doubles and phosphatidylethanolamine/ phospholipid shifts from $84 \%$ to $74 \%$ [481)]. Specific enzymatic desaturations, elongations and transacylations mediate phospholipid adaptations to low temperatures. Cardiolipins contain $66 \%$ cis-18: $1(n-7) .17 \%$ 16:0 acyls in $E$. coli growing at $40^{\circ} \mathrm{C}$ (Table II). The cis-3-unsaturaicd bond derives from the anacrubic dehydration of $\mathrm{D}$ - $\beta$-hydroxy-10:0-acyl-carrier-protein, an intermediate in the fatty acid synthesis pathway, to form either cis-10:1(n-7)-acyl-carrier-protein or trans-10: $1(n-8)$-acyl-carrier-protein (the conventional step in further elongation by malonyl-acyl-carrier-protein to form 16:(3-acyl-carrier-protein). Elongations corivert cis-10: $1(n-7) \rightarrow$ cis- $16: 1(n-7)$. $n$ cells growing at 40$)^{\circ} \mathrm{C}$. cardiolipins incorporate (transicylatc) this $16: 1$ acyl minimally (7\%; Table II), phosphatidylglycerols $18 \%$, and phosphatidylethanolamines $24 \%$ [360]; decreases in temperature do not affect these 16:1 acyl contents. Elongation of $16: 1(n-7) \rightarrow$ 18: $1(n-7)$ chains increases $15 \mathrm{~s}$ after temperature is decreased from $42^{\circ} \mathrm{C} \rightarrow>4^{\circ} \mathrm{C}$, through the activation of existing $\beta$-ketuacyl-acyl carrier protein synthase II. that catalyzes condensation of malonyl-acyl-carricr-protein with the growing fatty acyl chain [142]. This coldactivated enzyme is a translation product different from the less active, thermally insensitive synthase 1 . Transacylation of 18:1 acyls increases: in cardiolipins 18. I acyl content rises to $77 \%$ at $211^{\circ} \mathrm{C}$, displacing 16:0 acyls to 9\% (Table II); $18: 1$ acyls also accrue in phosphatidylglycerols $(41 \% \rightarrow 54 \%)$ and phosphatidylethanolamines $(24 \% \rightarrow 33 \%)$. Thus, cardiolipins play the most prominent but not the exclusive role in $E$. coli exponential-phase adaptation to low temperatures, by increasing their alrcady high contents of 18:1 acyls. Cardiolipins in stationary-phase E. coli, although much less unsaturatid $(7 \%)$ than in exponential-phase when growth is at $40^{\circ} \mathrm{C}$. triple their $18: 1$ acyl content at $30^{\circ} \mathrm{C}[360]$ (Table 11). The low 18:1 acyl contents of phosphatidylethanolamines and phosphatidylglycerols in stationary-phase cells grown at $40^{\circ} \mathrm{C}^{\circ}$ $(5 \%)$ increase cren more strikingly in cells grown at

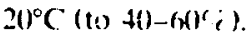

Changes in membrane physical and barrier properties accompany growth-temperature-induced adaptations in phospholipid fatty acyl compositions. Permeability (estimated from osmotic swelling) of liposomes prepared from extracted phospholipids is inverse to growth temperatures between 41$)^{\circ} \mathrm{C}$ and $21^{\circ} \mathrm{C}$ when $E$. coli is grown to the clid of exponential-phase [244]. Mean surface area of films made from total phospholipids of $E$. coli [244] or from phosphatidylethanolamines extracted frem Pseudomcinas fluorescens cells grown at $22^{\circ} \mathrm{C}$ is greater than in films from cells grown at $5^{\circ} \mathrm{C}[124]$. Thermal acclimation counteracts the enhanced molecular cohesion in monolayers at the lower temperatures [266].

When grown to stationary-phase at $40^{\circ} \mathrm{C}$ or at $20^{\circ} \mathrm{C}$. Mycobacterium hutyricum changes mainly the fatty acyl contents of cardiolipins [478] (Table II) but with less emphasis on elongations and desaturations ther: in $E$. coli. At either temperature, cardiolipin percentage of $16: 1=18: 1 ;$ at $40^{\circ} \mathrm{C} 16: 1+18: 1=46 \%$ of fatty acyls. at $20{ }^{\circ} \mathrm{C}, 82 \%$ of fatty acyl at the expense of $16: 0$ and 19:0-branched fatty acyls. At $40^{\circ} \mathrm{C}$, the phosphatidylethanolamines contain half as much monounsaturated fatty acyls $(23 \%)$ as cardiolipins: at $20^{\circ} \mathrm{C}$, phosphatidylethanolamine monounsaturated fatty acyls rise to only $32 \%$.

Ester-linked fatty acyl phospholipids are in plasma membran's of eubacticial thermophiles that remain stable at high tempciäiures, as do membranes with ether-linked phytanyl glycerols in archaebacterial thermophiles. The eubacterial moderate thermophilc Bacillus stearothermophilus grows optimally at $65^{\circ} \mathrm{C}$, the extreme thermophile Thermophilus aquaricus, at $90^{\circ} \mathrm{C}$. Cardiolipins in B. stearothermophilus cells in late exponential-phase are 30-40\% of total phospholipids and 50\%: of membrane phospholipids; phosphatidyl. glycerol/phospholipid and phosphatidylethanolamine/ phospholipid ratios are each about $25 \%$ [78]. Thermophile total phospho'ıpids contain all or almost all saturated, branched-chiin, isi- or anteiso-15-carbon and !? carton fatty aç ls. They contain no unsaturaied fatty acyls $[484]$ or $11.5 \%$ iso-17:1 [600]. The fatty acyls of the cardiolipins of $B$. stearothermophilus [78] and the extreme the:rmophilic bacterium PS3 [343] are all saturated (Table II); PS3 cardiolipin/phospholipid ratio is $19 \%$, phosphatidylglycerol/phospholipid, $13 \%$. The resistance of the saturated phospholipids of PS3 to heat and oxidation makes them useful in reconstituting the stable PS3 $F_{0} F_{1}$-ATPase complex $[343,618,619]$. Saturated cardiolipins are only $3 \%$ of total phospholipids of $T$. aquaticus in late exponential-phase or stationary-phase; $75 \%$ of to:al phospho- 
lipids is an unidentified phospholipid that contains three saturated fotty acvls, one giyecrol and an unsaturated long-chain amine [5.32].

Cardiolipins are $66 \%$ of the complcicly satuiaticd membrane phospholipids of Bacillus acidocaldarius, an extreme thermo-acidophile cubacterium [380] (Table II); proton conductance is in the range seen in other bacteria [372]. Right-side-out membrane vesicies pump respiratory-chain protons outwars and maintain an internal $\mathrm{pH}$ of 5.8-7.1 (cxtcrual pH being 2-3) as well as a reversed $\Delta \psi$, positive inside. Protonophores abolish niost of the $\Delta \mathrm{pH}$.

Cell membranes of $T$. aquaticus and $T$. thermophilus leak protons, mote rapidly as in vivo and in vitro temperatures are increased. $\mathrm{A}$ pH gradient generated in anacrohic $T$. thermophilus cells by an oxygen-pulse decays by proton leakage: when measured at $\left(50^{\circ} \mathrm{C}\right)$ the $t_{1 / 2}$ is $45 \mathrm{~s}$ in cells adapted by growth at $60^{\circ} \mathrm{C}, 19 \mathrm{~s}$ in cells adapted at $79^{\circ} \mathrm{C}$; when measured at $79^{\circ} \mathrm{C}, t_{1 / 2}$ is $\leq 8 \mathrm{~s}$ [425]. (Rat liver mitochondrial $t_{1 / 2}$ measured at $25^{\circ} \mathrm{C}$ is $8 \mathrm{n}-140 \mathrm{~s}$, and $5 \mathrm{~s}$ in the presence of FCCP $[437,438,459]$.) Despite the great proton leakage, rapid cell respiration maintains $\Delta p$ at $; 97 \mathrm{mV}$ (assayed at $70^{\circ} \mathrm{C}, \mathrm{pH} 7.5$ ), but growth efficiency is one third that of mesophilic bacteria that have an equivalent respiratory system (sec Ref. 184). 7. thermophilus cells at $70^{\circ} \mathrm{C}$ maintain an adenine nucleotide phosphorylation potential of $43.5 \mathrm{~kJ} / \mathrm{mol}$, and added FCCP depresses it to only $38.0 \mathrm{~kJ} / \mathrm{mol}$ : a high $\mathrm{H}^{+} / \mathrm{O}$ quotient must accompany the rapid oxidative generation of $\Delta p$.

ATPase-containing vesicles made from extracted saturated phospholipids of PS3 can attain a $J p$ of $\mathbf{3 1 0}$ mv at $45^{\circ} \mathrm{C}$; the $\Delta \psi$ component decays with a half-life of $55 \mathrm{~s}$. Half-life is $17 \mathrm{~s}\left(54^{\circ} \mathrm{C}\right)$ in less stable vesicles made from the naturally unsaturated soybean phospholipids.

Thermophilic bacteria grown to late exponentialphase at increased temperatures adapt ! iengthening their phospholipid carbon-chains and synthesizirig anteiso- or iso-fatty acids that have inigher melting points [78]; the fatty acyl composition of phospholipid subclasses are not reported. With cardiolipins containing saturated fatty acyls the membranes of thermophilic bacteria seem to support energy metabolism at high temperatures through a compromise between high stability and inefficient proton retention.

Effects of $\left[\mathrm{O}_{2}\right]$ : transition from anaerobiusis to aerobiosis leads to formation of the electron-transport chain while it increases cardiolipins and other phospholipids by $60 \%$ in Staphylococcus aureus [699] (Table II). However, only the cardiolipins reconstitute activity in the delipidated bacterial $D$-lactate oxidase, and (when they are not supplemented with $\mathbf{Q}_{10}$ ) in NADH oxidase and succinate oxidase $[177,424]$. Limiting $\left[\mathrm{O}_{2}\right]$ during $E$. coli growth at $30^{\circ} \mathrm{C}$ markediy decreases efficiency of energy conversion, as the cells synthesize $a_{1} a_{2}$ cy- tuchrome oxidases which may be associated with nonphosphorylating respiratory chains $[338,429]$.

ADP phosphorylation: colicin K, 2.4-dinitrophenol, cyanide, penicillir or ultraviolet radiation [572] dimin ish cell phosphorylating ahility while they increase the cardiolipin / phosphatidylglycerol ratio [629].

Protonophores: cardiolipins accumulate in bacteria grown in the presence of a protonophore. Cardiolipins in B. subtilis so grown comprise up to $70 \%$ of total phospholipids when extracted with adequately acidic solvents, but otherwise large amounts of phosphatidylglycerol are present as a cardiolipin breakdown product [376]. Phosphatidylglycerols are also a product of incomplete extraction in Streptococcus [189]. Cardiolipins accumulate in plasma membranes of Hemophilus parainfluenzae grown with СССР while the inighly active cardiolipin-specific phospholipase $D$ is blocked [481]. Since CCCP does not inhibit the isolated enzyme, the proton leak appears to depress cardiolipin catabolism. Conversely, blockage of any $F_{10}$-leak with oligomycin enhances cardiolipin hydrolysis.

Growth of the $E$. coli UVG strain in the presence of $0.25 \mathrm{mM}$ CCCP. as compared wit/i tts parent strain - CCC:, halves the phospholipid cuntent per me protein of the inner cell membrane; leaves proportions of cardiolipins, phosphatidylethanolamines and phosphatidylglycerols unchanged; and increases unsaturated fatty acyls $(16: 1(n-7)+18: 1(n-7))$ from $25 \%$ (-CCCP) to $45 \%$ with reciprocal decrease in saturated fatty acyls (plus cis-9,10-methylene-17:0) [272,583]. ATP/ADP ratios are maintained while content of ATP + ADP rises $50 \%$ and $P_{i}$ rises 13-fold. Increase in unsaturation of the membrane (we do not know fatty acyl composition of cardiolipins) might be viewed as an adaptative attempt to retain an effective $\Delta p$.

Mitochondrial origins: the present mitochondrion is thought to be derived from a successful symbiosis of an cukaryotic cell that performed anaerobic glycolysis, and a prokaryotic cell that had developed oxidative phosphorylation in a membrane, bascd on homologies between mitochondrial and prokaryotic DNAs, ribosomes and proteins [614]. From rRNA and cytochrome scquences, the most likely prokaryote endosymbiont is among the $\alpha$-subdivision of the photosynthesizing, purple, nonsulfur, Gram-negative bacteria [715,731]. Some $\alpha$-purple bacteria still associate intracellularly with various eukaryotes either as pathogens (e.g., agrobacteria for plants, Rickettsiae for animals) or as essential intracellular components, e.g., Rhizobacteriae for nitrogen-fixation in legumes [715]. Aerobic metabolism arose a number of times in $\alpha$-purple bacteria and early mitochendria may have metabolized vissuived $\mathrm{O}_{2}$ even before photosynthesis produced high atmospheric $\left[\mathrm{O}_{2}\right]$ [581].

Micrococcus denitrificans, in the a3-subgroup oi purple bacteria [715], has more mitochondrial features 
than other acrobic bacteria [331]: membrane phospholipids include an unusally high $31 \%$ of phosphatidylcholines, $52 \%$ are phosphatidyiglycerols unu $3.2 \%$ cardiolipins [701]; phospholipid fatty acyls are all straightchain; its cytochrome $c$ reacts with mitochondrial c $_{3}{ }_{3}$; its $P_{i}$-carrier is sensitive to sulfhydryl reactants. Everted particles from $\boldsymbol{M}$. denitrificans plasma membrane behave like mitochondria: respiration $\left(\mathrm{NADH}, 30{ }^{\circ} \mathrm{C}\right.$ ) is stimulated by addition of $A D P+P_{i}$ or $F C C P$, and depressed by the ATPase inhibitor venturicidin: $\mathrm{H}^{+} / \mathrm{O}$ ratios are comparable [329,330]. Rhodospirillum rubrum, in the al-subgroup of purple bacteria, has cardiolipins with relatively unsaturated fatty acyl groups that become slightly less unsaturated when photosynthesis is established (Table II).

Fatty acyl composition of cardiolipins is distinct from other phospholipids in several phyla: the saturated cardiolipins of $N$. coeliaca are characterized by very high contents of 16:0 acyls [734]; cardiolipins of $E$. culi and $M$. butyricum are more unsaturated than phosphatidylethanolanines and phosphatidylglycerols under similar growth and temperature conditions (see above); cardiolipins of $S$. cererisiae mitochondria are distinctively repleted in 16:1 and 18:1 fatty acyls (section IV-C.4); and cardiolipins from heart, liver or kidney mitochondria are enriched in 18:2 acyls (section IV-A). Thus, phospholipid fatty acyl compositions have undergone an evolutionary shift from completely endogenous saturated and monounsaturated fatty acyls in prokaryotes, to significant amounts of exogenous EFA (especially $18: 2(n-6)$ in caidiolipins) and their ( $n-$ 6)- and ( $n-3)$-polyunsaturated fatty acyls derivatives in animal mitochondria. $C$. crescentus can even incorporate into its cardiolipins $18: 2(n-6)$ fatty acyls added to the growth medium (Table II).

\section{Cardiolipins in eukaryotes}

Cardiolipins are synthesized in the mitochondrial inner membrane from one molecule of a phosphatidylglycerol and one molecule of a CDP-diacylglycerol. The liponucleoiides are produced from phosphatidate by either mitochondrial or microsomal CTP-specific transferases [305.320]. Cardiolipin linoleoyl acyls turn over faster than in other phosphclipids in liver mitochondria [378], probably as part of a remodeling that keeps the $18: 2$ content higher in cardiolipin than in its precursors. This remodeling appears to be a mitochondrial cycle not found in microsomes, that comprises phospholipase-A-catalyzed monodeacylation of newly synthesized cardiclipin, and a specific 18:2-CoA: monolysocardiolipin transfer(ase?) [578]. In contrast to the $18: 2$ acyls, the -P-glycerol-P-grouping turns over much more slowly in rat liver cardiolipins than the glycerol backbone of other phospholipids [378] and probably persists as long as the mitochondrial mem- brane itself, siace cardiolipin and mitochoncirial halflives are comparable [19i.2.32].

A corollary of the cuncept that ci.. lioipins exist in membranes that perform uxidative ohusphorylation would be that cardiolipins do not evist in obligatively anaerotic eukaryotes that have no mitochondria. Some protozoa have evolved without mitochondria [80]: Archamoebae, Metamonada, Microsporidia and Parabasalia. Giardia lamblia, an archezoan metamonad with no mitochondria, two nuclei and DNA of early evolutionary origin, is a recently proposed candidate fo: the anaerobic eukaryote that was the first host for an atrobic prokaryote among the $\alpha$-purple eubacteria [340]. It now lives parasitically in animal and human intestinal tracts and depends on preformed biliary phospholipids and cholesterol for phospholipids and fatty acids, which it does not readily synthesize. Bile phospholipids do not include cardiolipins [183]. Giardia phospholipids incorporate saturated fatty acids and 18 : I acid from the growth medium [327,344], but when external $18: 2$ acid is $56 \%$ of fatty acids the phospholipids contain only $4.5 \%$ 18:2 acyls [344]; this would be consistent with the absence of cell cardiolipins. No cardiolipins are reported in $G$. lamblia membranes $[42,327,344]$ or in another amitochondrial anaerobe, Entamoeba incadens [426,667], in phospholipid analyses that usually detect small quantities of cardiolipins, although some unidentified phospholipids were seen. Presumably mtDNA codes for the enzymes that synthesize cardiolipins. Descendent eukaryotes of such amitochondrial anaerobes would thereby have no cardiolipins in their plasma membrane phospholipids.

\section{IV-A. Cardiolipin / phospholipid ratios and fatty acyl composition}

Analyses for tissue cardiolipins and phospholipids should separate phospholipid subclasses completely and non-destructively. However, the usual extraction methods may not recover all the cardiolipins of beef heart $[612,716]$ or the cardiulipins of some stationary-phase bacterial celis (section III-A). Some mitochondrial cardiolipins can readily be peroxidized, polymerized or deacylated. Cardiolipins in various vertebrate tissues, especially heart, are preferentially deacylated by an endogenous phospholipase $A$ [242]. On the other hand, liver cardiolipins ar: not especially sensitive to oxidation with ascorbate, which decreases unsaturated fatty acyls in all phospholipids [395, 272$]$.

Tissue concentration of cardiolipins (per amount of tissue, DNA, total phospholipids or protein) measures the cell content of mitochondrial inner membranes [326] and therefore correlates with tissue total oxidative capacity. The ratio cardiolipin per mitochondrial total phospholipids, and the percentage distribution of 


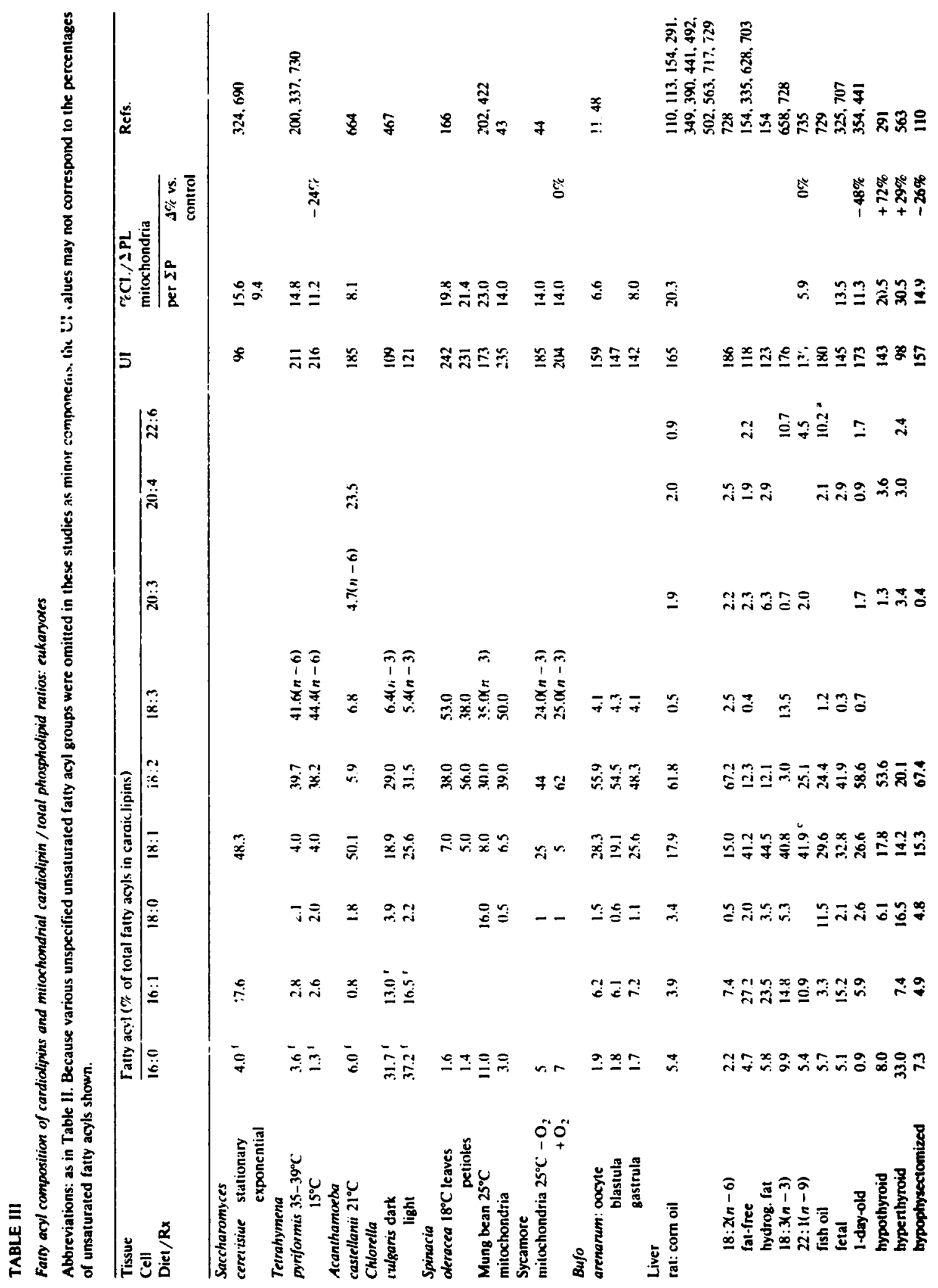


辛

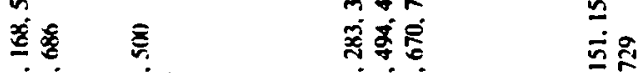

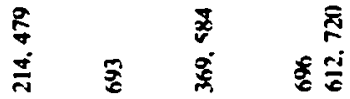

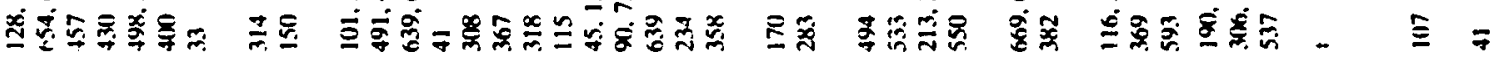

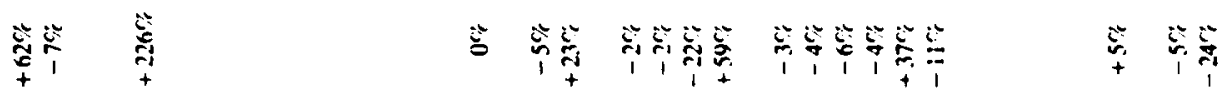

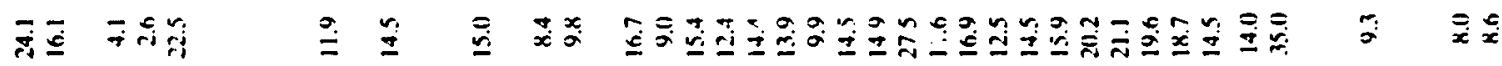

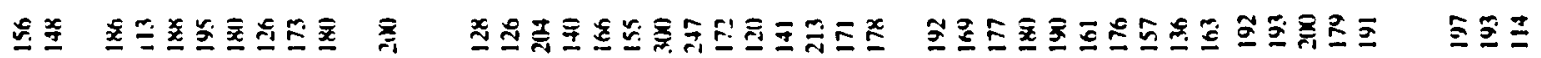

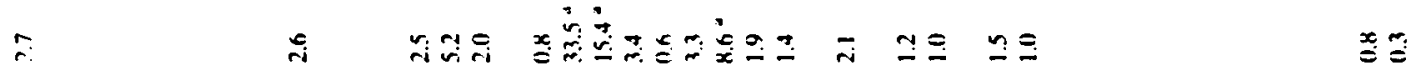

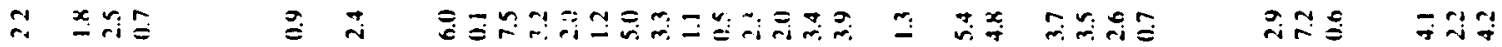

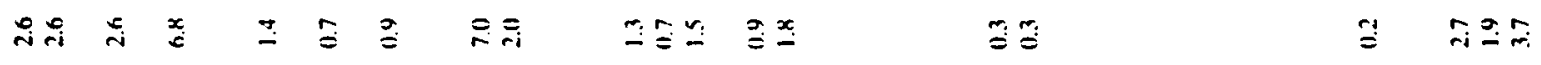

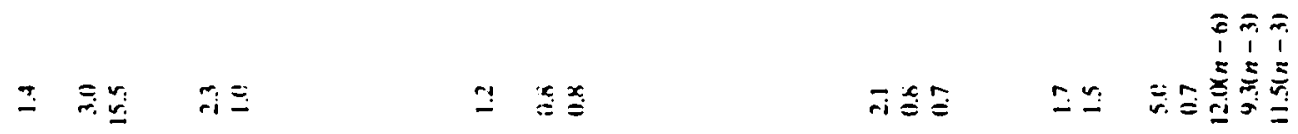

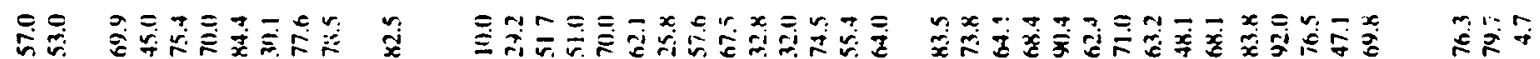

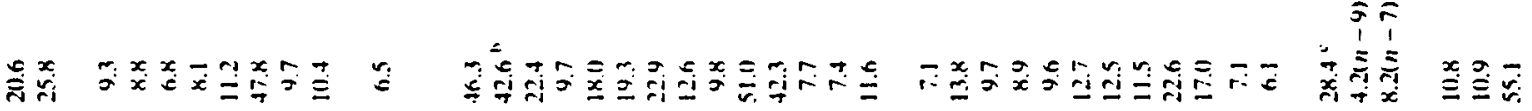

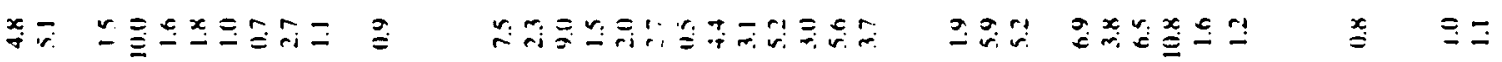

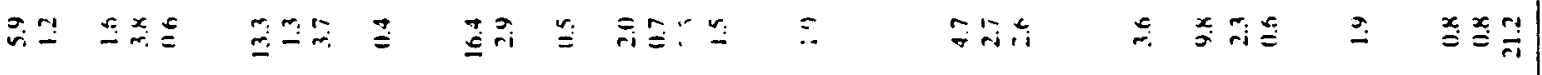

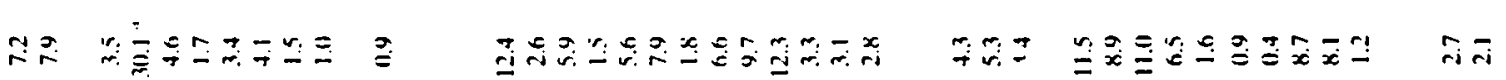

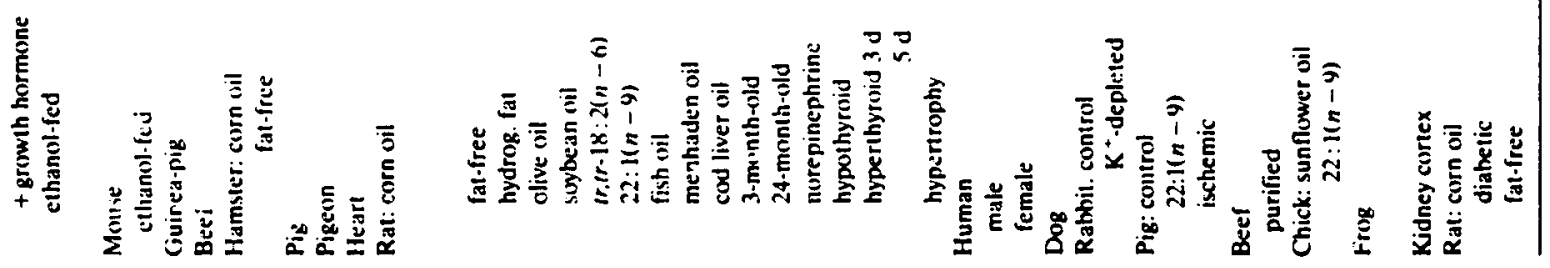




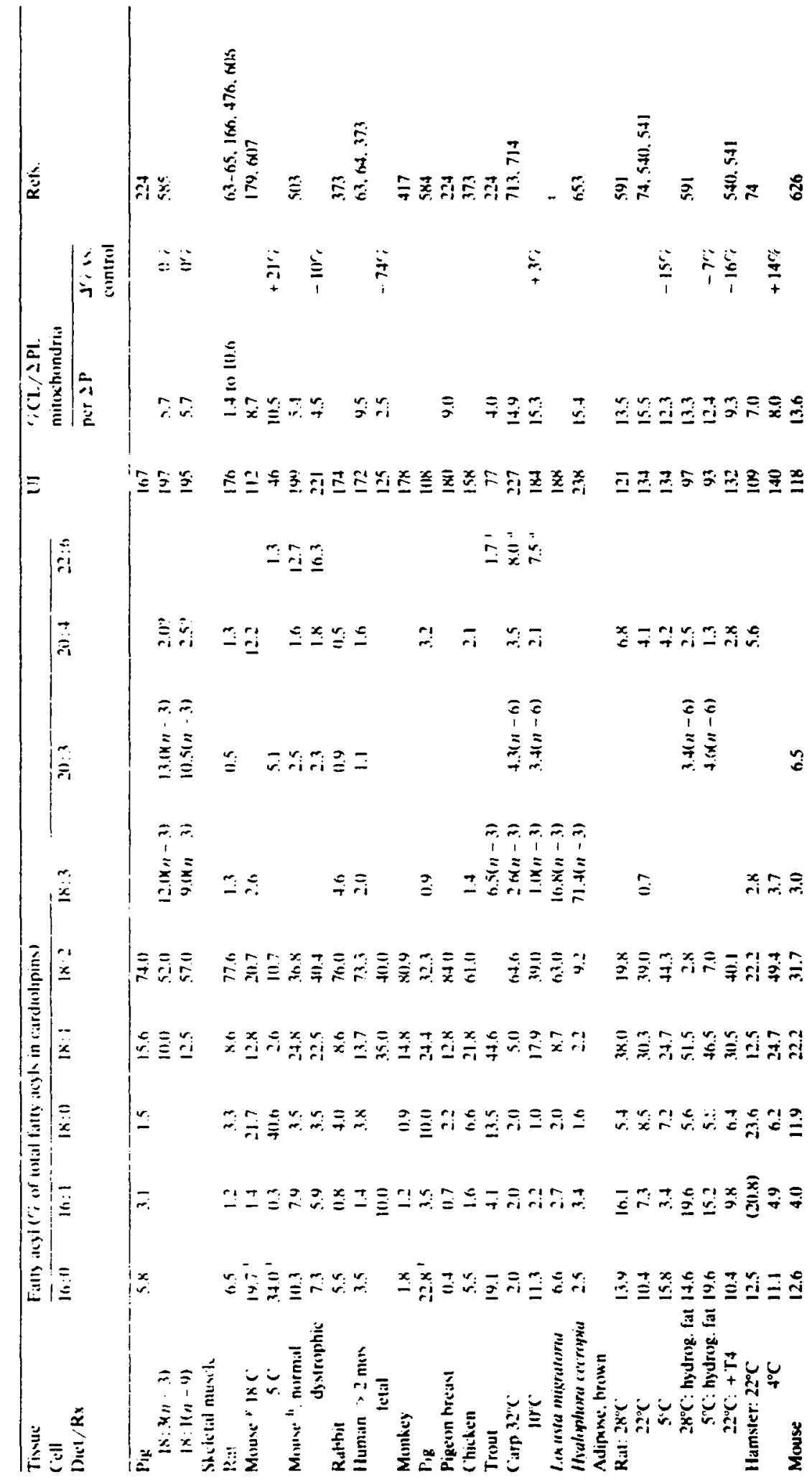




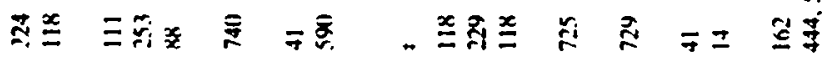

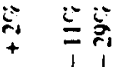

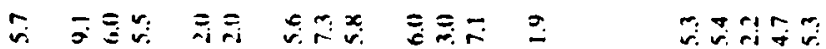

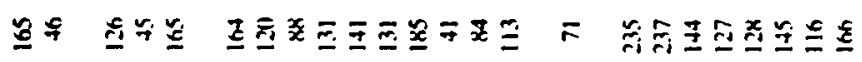

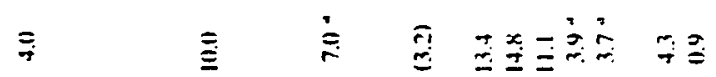

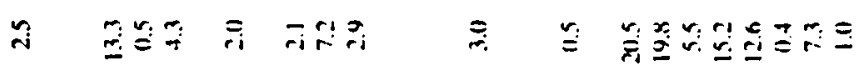

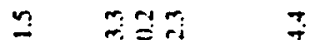

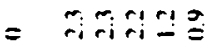

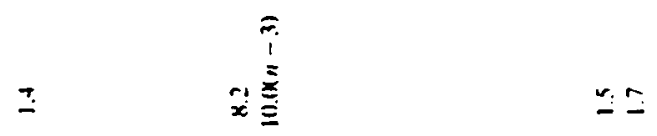

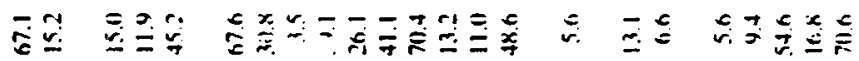

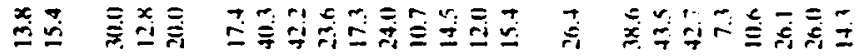

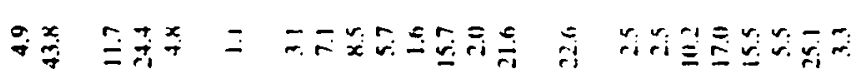

手

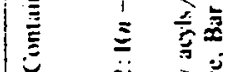

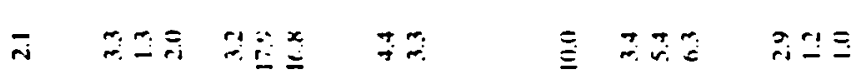

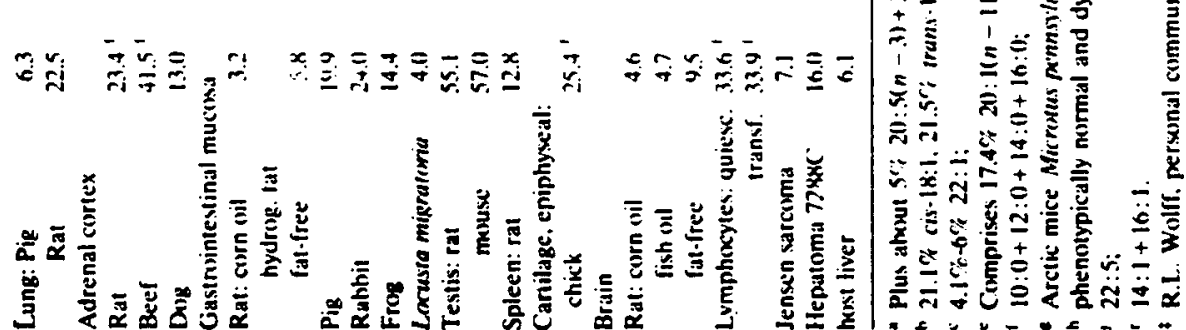


fatty acy' groups in cardiolıpins. measure inner mesnbranc composition, and appear to correlate with specific oxidative activity. based on evicence discussed here and elsewhere. Cardiolipin P per total phospholipids P shows the cardiolipin contribution to the total fatty acyl groups of the membrane. since there are two acyls for each $P$ atom of either cardiolipins or other phospholipids. Mol-fraction of cirdiolipin/total phospholipids denotes the contribution of the -P-glycerol-P. grouping of cardiolipins to the polar groups of the membrane.

By the usual chloroform/methanol extract ions, rat liver mitochondria contain some $3(x)-5(0)$ nmol of phospholipids per mg protein [386]. Wc found 19() nmol of total phospholipids per $\mathrm{mg}$ protein. including 11 nmol of cardiolipin $P$ per mg [24]). Cardiolipin P per phospholipid $P$ ratios are 10-25\% in most tissucs [136, 19],3015,697] (Tahle 111).

As much as 35\% cardiolipin P per phospholipid $P^{\prime}$ can be recovered from beef healt [012]. Comtanis itf cardiolipins are of the same order as matrix adenine nucleotides, pyridine nucleotides and perhaps divalent cations. and several orders greater than the amounts of matrix proteins. Little attention has heen given to variations in the small amounts of cardiolipin on the outside layer of the inner membranes, which determine specific binding to certain proteins.

\section{IV-B. Natural iariation}

Animal cardiolipins vary widely in falty acyl distribution, contrary to statements that cardiolipin normally is very homogeneous, with 60-80\% linolonyl chains [89,582]. Some physiological variations in amounts of cardiolipins per amount of tissue [136] and in percentage fatty acyl distributions [320] have been revicwed. Several in vivo factors are associc...ed with distinctive natterns of cardiolipin fatty acyl compositions and cardiolipin/p'rospholipid ratios (Table II!) development and aging, tissues and species, diet fatty acids, ethanol feeding. Mg-deficiency. environmental temperature, aitered hormonal states and pathological conditions. In some cases, mitochondrial respiration varies with cardiolipin compositions.

\section{IV-B.I. Development and aging}

The life-cycles of various prokaryotes involve lipid changes. During embryogenesis in Amphihia, from oocyte $\rightarrow$ gastrula. mitochondrial cardiolipin/phospholipid ratios increase minimally while cardiolipin fatty acyl composition and mitochondrial function do not change. After gastrulation. State 4 respiration and mitochondrial replication acceleratc. In fetal $\rightarrow$ postnatal development of rats, and perhaps during maturation to adults ( 4 months), cardiolipins and mitochon- drial oxidaive phosphorylation change greatly. Changes in senescence: ( $>30$ months in rats) have elicited much less agrcement among obseners.

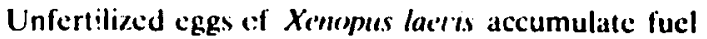
and matabolite precursors and store $10^{7}$ mitochondria per cell to he used during embryogenesis - the oncytes contain $100^{5}$ more mitochondria than somatic cells [91]. The egg cell contributes all the mtDNA to the fertilizcd zygote: mitochondrial inheritance is maternal in Xenopus [137] and in the higher animals including humans $[212,6(1) 2]$. Most of the human miDNA codes for polypeptides in complex $I$ and cytochromes $b$ and $\mathrm{Aa}_{3}$ (see Ref. 75). Embryogenesis in nonmammalian species involves rapiud cell division of the large fertilized oxkytc, without increase in total mass. The fertilized egg divides to 64 cells in $4 \mathrm{~h}$. $10^{4}$ cells at $6 \mathrm{~h}$ (hlastula) and $3 \cdot 10^{+}$cells at $10 \mathrm{~h}$ (gastrula) [6]. Oxcytc mitochondria ati dilued among daughter cells until gastrulation. when $1 \mathrm{cu}$ mitconondral r.RNA synthesis starts [1)].692]: oxcyte cardiolipins are conserved in enthryogenesis.

Cardiolipins in miokhondriat of unfertilized eggs of the toad Bufo arenarum ate atheut $7 \%$ of total phospholipids and have characteristically high contents of $18: 2(5.5 \%)$ and $18: 1$ (28\% $)$ acyls $[11,48]$ (Table lil). Cardiolipin/phospholipid proportions increase slightly while cardiolipin fatty acy! composition remains constant during development from fertilized egg to gasirula stage. Mitochondrial cardiolipins increase ${ }^{32} \mathrm{P}_{\mathrm{i}}$ uptakc minimally up to gastrulation, whereas phosphatidylcholines, phosphatidylethanolamines and phosphatidylinositols turn over progressively faster [11]. Mitochondrial function up to gastrulation has adult characteristics in terms of relative rates of substrate oxidation, and inhibitor patterns [385,568], hut calculisted Statc 4 respiration $\left(25^{\circ} \mathrm{C}\right)$ is only about $2 \mathrm{ng}$ atom $0 \mathrm{~min}^{1}$ ( $\mathrm{mg}$ mitoxhondrial protein) "'. State 4 accelerates 4-fold after gatstrula stage: the new mitcchondria leak protons faster than their maternal precursors.

Ryuzaki [5(.7] repo,ts that relative amounts of phosphatidylcholines $(5() \%)$. phosphatidylethanolamines (20ri) and phosphatidylinositols $(1-2 \%)$ remain constant in the post-gastrula embryonal development of Rana nigromaculata to the tadpole stage. Chromatograms of egg and embryo lipids show progressively greater amounts of unidentified frontal phospholipids (usually cardiolipins and phosphatidate) which seem to be some $25 \%$ of total phospholipids in early tadpole stages.

Development: the liver, its membranes and its lipids restructure remarkably during natural or thyroid-induced Anuran metamorphosis. Hepatic synthesis of phospholipids in endoplasmic reticulum and mitochondria becomes rapid in > 4-day-old Xenopus larvae, when T3-binding appears [641-643]. In endoplasmic reticulum, the phospholipid-subclass ratios persist. 
The phospholipid/neutral lipid ratio is 0.5 in livers of $k$. cateshiaria tadpoles, 3 in frugs $\left[282.28^{\prime \prime}\right]$ and 1.5 in $\boldsymbol{R}$. esculenta frogs [?(n)! Organelle membranes are vesicular in tadpoles, bilamellar in frogs [6+11]. Tadpole liver totai lipids contain $30 \%$ polyunsaturdted fatiy acyls, 45\% monounsaturated fatty acyls; heart lipids have $30 \%$ polyunsaturated fatty acy's. $25 \%$ monounsaturated fatty acyls. Frog livers have high polvunsallirated fatty acyl contents [29,282,287]. 'The linuleoyl acyls in Rana esculenta livers are exogenous, since 18:2 is essential [29]. Both tadpole and frog livers convert $18: 2$ to $(n-6)$-polyunsaturated fatty acyl derivatives very actively. but during metamorphosis phospholipids are synthesized that retain the poly. unsaturated fatty acyls produced - perhaps cardiolipins: we have no measurements. Cardiolipin/phospholipid ratios are $2 \%$ in frog liver and skeletal muscles, $5 \%$ in kidney and $9.3 \%$ in hearl [561,6(k).7(m)]. To the extent that the cardiolipin fatty acyl composition of ocytes represents that of simatic cells, adult Bufo cardiolipins have high $18: 2$ acyl contents like rat liver mitochondrial cardiolipins. nut higner contents of $1 \mathrm{~h} . \mathrm{i}$ and significar! amounts of an $18: 3$ acyl.

Cardiolipins in insect flight muscles increase line: rly and strikingly over the 5-9 days when pupae deveiop into adults: from $7 \%$ to $20 \%$ of mitochondrial phospholipids in tobacco horn worms [85] and from $1.3 \%$ to $12 \%$ in $H$. cecropia [653]. The highly unsaturated cardiolipins of Cecropia adults contain $71 \%$ 18: $3(n-3)$ acyls derived from the phytophagous habits of these insects. plus $9 \% 18: 2$ acyls and $6 \%$ monoun. saturateo fatty acyls (Table III).

Cardiclipin contents of livers [325] and kidneys [394] of fetal r.ts are half of adult levels, and in fetal human skeletal inuscles, one quarter [6.3-65]: fetal cells contain few mitochondria. In addition, the cardiolipin/ phospholipid ratio in liver mitochondria of fetuses is less than half that at 3 weeks after birth [390,441]: fetal mituchondria have low cardiolipin contents. The relative rates of cardiolipin synthesis are, respectively, $1: 4$ in heart mituchondria from adult and neonatal rats. $11: 2 ?$ in liver mitochondria of neonatals and adults [635].

Fetal tissucs also have few 18:2 acyl groups. The 6\% of 18:2 acyls in liver phospholipids of 1 - and 5-day-old rats doubles by day 15 and triples by day 25 and in adults [589]. Fetal rat liver cardiolipins contain 40)-60\% 18:2 acyls and 30. 50\% monounsaturated fatty acyls, adult liver cardiolipins have $60-80 \% 18: 2$ and $20 \%$ moncunsaturated fatty acyls $[354,441,707]$. Even more strikingly, skeletal muscle cardiolipins of human fetuses are $40 \%$ in $18: 2$ and $45 \%$ in monounsaturated fatty acyls, which shifts to $90 \% 18: 2$ and less than $10 \%$ monounsaturated fatty acyls by 2 months after birth [63-65]. Low fetal 18:2 contents reflect a cardiolipindeficit or an inability to enrich cardiolipins with $18: 2$ acyls. Although the pattern resembles that in EFA-deficiency, phosphatidylcholines and phosphatidylethanolamines contain as much $18: 2$ in fetal as in adult livers [4+1): adult EFA-deficiency depletes $18: 2$ acyls in these fraciions (sec section IV-C.1.a). In liver mitochondrial cardiolipins of 1-day-olu rats. 18:2 acyls are $48 \%$ of total fatty acyls, $58 \%$ on day $3,63 \% ;$ on day 15 and 79\%; (adult level) on day 25; 18:1 acyl contents decline reciprocally [4+1]. Thus, linolcoyl acyls replace monounsaturated fatty acyis during suckling more slowly than fed $18: 2$ acid reverses EFA-deficiency in adult rats (Table III), perhaps because the necessary enzymes must develop.

Mitochondria from livers of fetal (term) or 1-day-old rats leak protons under State 4 conditions up to twice faster than do those from adult rats. and still phosphorylate ADP efficiently [25.510]. State 4 respiration halves between day 2 and 9; slow proton-leakage and the adult cardiolipin high-18:2, low-monounsaturated fatty acyls profile develop in parallel. In contrast to adults, the major regulators of State 3 respiration in

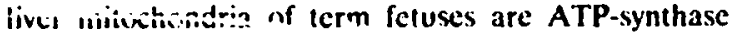
and $P_{i}$-carrier, and the dicarboxylate carricr and ADP/ATP carrier are less important [25] (see section :I-B). Mitochondria fron maturing rat brains also leak protons, but transiently. State 4 respiration is very slow in the first weck postpartum, 6-9-fold faster in weeks 2 and 3, and less rapid thereafter [2\%]. Maturation of rat brain depends on adequate thyroid hormone levels, and fetal hypothyroidism produces damage that is not reversec by thyroid administration afi.r week 2 postpartum .225].

Chondrocytes develop in epiphyseal growth plates boih pre- and postnatally. The fatty acyl patterns of membrane pnuspitsilipids of growth plate cartilage cells from young chicks, futal calves or newborn pigs resemble those of livers troir, EFA-deficient animals, although the phospholipids of their muscles, liver, bones and sera show normal fatty acyl patterns $[4,5]$. Chondrocyte membrane levels of $18: 2(n-6)$ and $20: 4(n-$ 6) acyls are very low, amounts of monounsaturated fatty acyls are high, and Mead acid $20: 3(n-9)$ appears in quantity is phosphatidylethanolamines and phosphatidylinositois. With aging, the cartilage of weight-bearing joints linearly accrues $(n-6)$-unsaturated fatty acyls that displace the $20: 3(n-9)$ acyls. An apparent localized EFA-deficiency in developing cartilage has been attrihuted to (i) a limited access of plasma EFA-albumin complexes to cartilage cells caused by the dense impermeable matrix of proteoglycans and collagen that also seems to slow amino acid exchanges; (ii) the observed rapid metabolism of $18: 2$ and 20:4 acyls in growth plate cells: and (iii) the high activity of chondrucute 49-desaturase. Cardiolipins of isolated epiphyseal chondrocyte membranes prepared with collagenase digestion comprise only $1.9 \%$ of phos- 
pholipid ') $[725,726)$ : chondrecyles contain icw mitochondria. Cardiolipin fatty acyls in chich chondrocyte membranes are mostly nonounsaturated fatty acyls (36\%) and saturated fatly acyls $(48 \%)$, whilst $18: 2$ acyls are only 5.9\%: unsaturation index is 71 and no 20:34n 1) fatly acyls are present [725] (Table III). This pattern is seen in cardiolipins of other developing cells in fetal liver and in iepatomas (Table III); in contrast, in EFA-deficiency 18:2 acyls are defleted, only menounsaturaied fatty acyls are high. and liver cardiolipin / phospholipid ratios are normal.

Oxidative phosphorylation in chondrocyte mito. chondria apparently depends on the preparative methods, which are difficult becausc cartilage is dense and has few mitochondria. Thick chondrocyte mit'nchondria prepared by high-speed homogenizations and differential sedimentat ons do not leak protons excessivcly: State 4 respiration (succinate, $26^{\circ} \mathrm{C}$ ) is $40-53 \mathrm{ng}$ atoms $O \mathrm{~min}{ }^{\prime} \mathrm{mg}{ }^{\prime}[3 \times 3,3 \times 4]$. as in normal liver mitochondria (Table VIII). Thus, these low-linoleale-content cardiolipins do not accelerate proton leakage. perhaps hecause they are so sparse - which implics that normal proton leakage can depend on phosphatidylcholines and phosphutidy lethanolamines. Mitochondrial $\mathrm{Ca}^{2}$ in cartilage is $10-30$-fold that in soft tissues like liver; mitochondria from the hypertrophic 7 ne of the growth plate have about $80 \mathrm{nmol} / \mathrm{mg}$ protein and those from the calcifying zone about $3(0)$ nmol/mg [594]. In the naturally Ca" -loaded cartilage mitochondria, ADP $+P_{i}$ proxluce Statc $4 \rightarrow 3 \rightarrow 4$ transitions. In liver mitochondria loaded with this much $\mathrm{Ca}^{2+}$, and in mitochondria from Ehrlich ascites tumor cells, ADP $+P_{1}$ stimulate a continued rapid respiration (proton cycling); hepatoma (ardiolipins also have low 18:2 and high monounsaturated + prlyunsaturated fatty acyl contents (sec Table III).

Mitochondria have also been prepared from calf growth plate chondroittes by pressuri-disruption, sequential digestions with EGTA. Irypsin, hyaluronidase and collagenase, and centrifugations [627]. Mitochondria in these cells are $4 \%$ as many, and have about the same $\mathrm{Ca}^{2+}$ content, as in guinea pig hepatocytes. Rates of respiration ( $\mathrm{ng}$ atoms 0 min $1 \mathrm{mg}$ ") plus succinate (rotenone, $P_{1} ?, 37^{\circ} \mathrm{C}$ ) are $58 ;$ + ADP (State 3), 70; + oligumycin (i.e., State 4), 34; + $\mathrm{Ca}^{2+}$ or uncoupler, about 125. Thus, blockage of the $\mathrm{H}^{+}$-ATPase inhibits the apparent State 4 proton-leak by $40 \%$ - as it did in the guinea pig liver mitochondria these workers used for comparisons. However, the State 3 rate in the liver mituchondria was 226 and the uncoupled rate was 290 . These chondrocyte mitochondrial phospholipids do not appear to leak protons excessively but the $\mathrm{H}^{+}$-ATPase does; the mitochondria were thought to be specialized for $\mathrm{Ca}^{2+}$-transpori rather than ADP phosphorylation.

Maturation in rats fed a high-fat diet $(20-40 \%$ of (alories as soy hean oil) from age 21 days to 4 months is accompanice by increased heart mitochondrial cardiolipin P per total phospholipids P (18\% before, 22\% after) and diminished cadiolinin 18:2 content [319] (lable III). These changes in inuer membrane lipids arc attributed to aging rather than diet and are thought to account for observed alterations in thermoiropic propertics and decreases in State 3 respiration and ATP-P, exchange [106].

Senescence in laboratory rats is accompanied by depletions: in membrane phospholipids, in unsaturation of fatty acyls, and in cardiolipins and phosphatidylethanolamines among the mitochondrial phospholipids. Such changes have been measured in liver, kıdney and heart phospholipids. plasma membranes, ritochondria and miciosomes [227.270,471,473,545, $592.64(1,682.6 \times 3.3$. Usually siturated fatty acyl contents increase reciprocally. monoene acyls remain unchanged, and 20:4 acyls decrease; $18: 2$ and $22: 6$ acyl contents are repleted or depleted in different reports. Because 201:4 acyls are a desaturation product of $18: 2(n-\theta)$, and $22: 6$ acyls derive from fed $18: 3(n-$ 3). the otserved decrease in fatty acyl-CoA J6-desalturase activity with aging [59] has been proposed as an important aging target [297]. A defect in 59-desaturations [196,204,205] is not accompanicd by diminished membrane contents of $18: 1$ acyls.

l.iver, heart and kidney mitochondria of senescent rats contain 25\% less cardiolipin per total phospholipids than those from young rats. In liver, less cardiolipin may be synthesized because activity of the mitochondrial CTP:phosphatidate cytidyltransferase decreases [682]. According to Jakovcic et al. [326], decreascod cardiolipins are associated with lessened inner membranc area. Liver mitochondria from old rats have smaller, sparser cristac in situ [644], and increased lipid structural order [682,683] and fragility [694].

Cardiolipin fálty acyl compositions in senescence have been described in two studies on rat heart mitochondria [359.391] (sce Table III). In one report. young-rat cardiolipins contain 1.3\% $18: 2$ acyls (sic) and 50 i) saturated fatty acyls while old-rat cardiolipins have 19.6\% 18:2, 40\% saturated fatty acyls; the other phosnholipids are similarly very low in polyunsaturated fatty azyls and high in saturated fatty acyls [391]. In the other study, mitochondrial cardiolipins from either young or old rats contain $32 \% 18: 2$ acyls and about $50 \% 18: 1$ acyls [359]. The 23\% diminution in $18: 2$ acyls of total mituchondrial phospholipids with aging is not reflected in the resolved cardiolipins, phosphatidylcholines, or phosphatidylethanolamines: some 18:2 acyls have been lost. Comparisons with heart cardiolipins of adult rats in Table III. which contain more than $80 \%$ 18:2 fatty acyl groups and more than $50 \%$ of the total mitochondrial $18: 2$ acyls, indicate that cardiolipins in both these studies have been extensively oxidized 
during extraction and. analysis. It seems likely that senescence does deple: 14:2 acyls in hedrt cardiolipins, from the 15-37\% iuss of $18: 2$ acyls in mitochondrial ph.spholipids [359.474.406] - hut we do not know what acyls replace them. Loss of cardiolipin 18:2 acyls has been altribuicd to increased peroxidative reactions with senescence [270,473]. which should selectively destroy membrane polyunsaturated fatty acyls through mitochondrial and microsomal byproducts of $\mathrm{O}$, metabolism. However, although inner mitochondrial membranes from 14-month-old rat hearts generate more superoxide radicals than those from 3-month-old rats, no further increase occurs at 24 months [451]. Another mechanism might be a progressive loss of preventive reactions that scavenge free radicals [256].

Senescence generally slows both State 4 and State 3 respiration in various mitochondria (Table IV). State 4 and State 3 do not decrease equally in different studies: Hansford [258] finds no change in State 4 respiration. Even though these disparitics make resu. int respiratory control ratios irrelevant to senescence mechanisms, some workers conclude that oxidative phosphorylation is not grosily impaired in mitochondria from senescen: animals because respiratory control ratios do not decrease [216,257,412]. The constancy or decrease of State 4 respiration with advanced age indicates that proton leakage does not increase. or even decreases. The variahility in findings on State 3 respiration in senescent animals may well reflect the sensitivity of control coefficient values to experimental cinditions [228], and the fact that different carriers regulate in liver and heart (and prehahly other) mitochondila. which may be why senescence has tissuc- and substrate-specific effecis on State 3 respiration (Table IV). A decrease in activities of carriers depresess respirittion, not through changes in carrier molecules or amounts hut by decreased matrix pools of exchangeable metabolites and by (perhaps related) altered inner membrane phospholipids [256,257].

In liver mitochondria, senescence decreases the ADP/ATP carricr $V_{\text {raax }}\left(\right.$ ADP. $4^{\circ} \mathrm{C}$ ) by $32 \%$, which seems attributable 10 the $28 \%$ diminution in matrix content of ATP + ADP (the exchangeable nucleotides) and the $20 \%$ decrease in the rate of actual ATP exchange; concomitantly, the carrier $C_{i}$ gains a value of 0.5 , compared with 0.28 in mitochondria from young rats $[135,228,358]$. State 3 respiration (succinate, $30{ }^{\circ} \mathrm{C}$ ) slows hy $20 \%$. Cytochrome $a a_{3}$ is a minor regulator of State 3 oxidation. with $C_{1}=0.11$. in liver mitochondria from cither old or young rats [1.35]; $C_{1}=0.17$ in young rats [228]. This is consistent with the constancy of [heme $a a_{1}$ ] in liver mitochondria from old and young rats. Li : 2 rs from old rats contain about $30 \%$ less $a a_{3}$ than thuse from young rats [683]. which indicates fewer mitochondria. Although the dicarhoxylate carrier is the other major regulator of State 3 respiration in young rat liver mitochondria, with $C_{i}=0.33$ [228], no measurements are available of its contribution to slowed succinate oxidation in senescence.

In heart mitochondria, serescence alters sedimentation behavior [449] and Arrhenius profiles of the $F_{1} F_{10}$. ATPase, the dehydrogenases specific for succinatc, glu-

TABLE IV

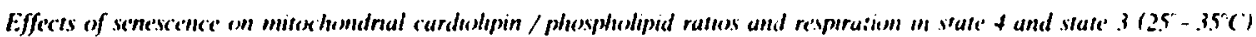

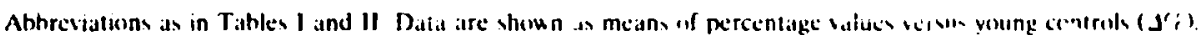

\begin{tabular}{|c|c|c|c|c|c|c|}
\hline \multirow{3}{*}{$\begin{array}{l}\text { Mito- } \\
\text { chonditia }\end{array}$} & \multicolumn{2}{|c|}{ CL./IPI. } & \multicolumn{4}{|l|}{ Respiration } \\
\hline & \multirow[t]{2}{*}{5} & \multirow[t]{2}{*}{ Rets. } & \multirow[t]{2}{*}{ substiatc } & \multicolumn{2}{|c|}{ J': Slate } & \multirow[t]{2}{*}{ R.fs. } \\
\hline & & & & 4 & 3 & \\
\hline \multirow[t]{3}{*}{ iver } & .23 & 358.682 & succinalc & 3 & -16 & $\begin{array}{l}135.311 .312 . \\
35 \times .6 \times 2\end{array}$ \\
\hline & & & glutimate/malate & -13 & -32 & 12. 3i2 \\
\hline & & & $\beta$-hydroxyhutyrailc & -25 & -12 & 216 \\
\hline \multirow[t]{5}{*}{ Heart } & -26 & $\begin{array}{l}227.359 .341 . \\
474.446\end{array}$ & succinate & -12 & +5 & 1.35 \\
\hline & & & glutamate/malate & -13 & -111 & $12 . \% .1112 .451$ \\
\hline & & & py :uvate & -20 & -22 & 359.546 \\
\hline & & & $\beta$-hydroxybutyrate & -20 & -18 & 216 \\
\hline & & & palmitoylcarnitine & (1) & $-\geq 1$ & \% \\
\hline Kidncy & -20 & 227 & $\beta$-hydroxybutyrate & -24 & -21 & 216 \\
\hline Muscle & & & glutamate /malate & -8 & -19 & \% \\
\hline \multirow[t]{3}{*}{ Brain } & & & succinate & $-\boldsymbol{y}$ & -4 & $\begin{array}{l}96,148,149 . \\
256,681\end{array}$ \\
\hline & & & glutimate/malate & -41 & -30 & \\
\hline & & & pyruvate /malate & -23 & -27 & \\
\hline
\end{tabular}


tamate, and $\beta$-hydroxybulyratc, and the succinate ixidase [471.472]. In vitro application of detergent restores normal activity-temferature relationships and (thereby) restores depressed rates, which further indicates that altered membrane lipids, rather than proteins, are responsible [471]. Senescence depresses State 4 respiration when glutamate/malate, nyruvate or $\beta$ hydroxybutyrate (but not palmitoylcarnitinc) is oxidized (Table IV). With succinate, $O$,-pulsing under State 4 conditions produces similar $\mathrm{H}^{*} / \mathrm{O}$ ratios (proton-slip) in young and old [412], indicating that proton permeability diminishes with age.

In heart mitochondria from old rats, as compared with young rats, respiration under State 3 conditions slows when glutamate/malate, pyruvate, $\beta$-hydroxybutyratc or palmitoylcarnitine (but not succinate) are substrates (Table IV). Depressed activities of several carriers in the inner membrane are involved in this dewn-regulation. (i) ADP/ATP carrier rate depression should affect all subsiralc exidations. Without measurements of $C_{i}$ it is difficult to assess how immortant obseived 40\% decreases in senescent rat heart mitochondrial ADP / ATP carricr activity at $6^{\circ} \mathrm{C}$ [474] are in the slowing of State 3 respiration at $\geq 25^{\circ} \mathrm{C}$. This carrier deses not regulate in hearts of adult rabhits [355.356] (see Table 1). although it does in liver mito)chondria. Old-heart mitochondria have as many available ADP/ATP carrier sites as young mitochondria. but matrix ATP + ADP is $20 \%$ less $[359,474]$. Nohl and Krämer [474] attribute decreased activity 10 decreased cardiolipin/phospholipid and phosphatidylethanolamine/phosphe,lipid ratios that depress membrane fluidity. Kim et al. [359] downplay any role of malrix ATP + ADP concentrations because adenine nucieotide depletion is less severe than in liver mitochondria, and the progress of the depletion does not correlate with the depression of myccardial respiration.

(ii) Monocarboxylate carrier. Sencscence slows pyruvate oxidation under Statc 3 conditions $\left(25^{\circ} \mathrm{C}^{\circ}\right)$ in heart mitochondria (Table IV). The pyruvate transporter in heart mitochondria from young rats contributes to the

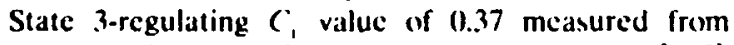
rotenone titrations of the NADH dehydrogenase [155]. and pyruvate transport limits pyruvatle oxidation in rat heart (but not liver) mitochondria [596]. Senescence slows pyruvate uptake at all temperatures between $4^{\circ} \mathrm{C}$ and $2 x^{\circ} \mathrm{C}$. and shifts the transition lemperature in Arrhenius profiles from $16^{\circ} \mathrm{C} \rightarrow 21^{\circ} \mathrm{C}$, without changing the number of available carrier sites or $\mathrm{JpH}$ this transporter responds sensitively to $\mathrm{JpH}$ across inner membrane). These findings suggest that the observed changes in fatty acyl composition of phospholipids and occrease in cardiolipin/ phospholipid affect the carricr activi!y tirrough specific binding to cardiolipins. (iii) The pa!mitcylcarnitine-carnitine translicase $V_{\max }\left(5^{\circ} \mathrm{C}\right)$ decreases by $35 \%$, and State 3 oxidation diminishes by 27ri (Table IV) in heart mitochondria of senescent rats [257]. Translocase first-order rate constant for exchange remains constant, but matrix contents of carniline auis exchanged-carnitine are $355^{\prime} ;$ and 22\%, respectivel; of the youthful values: the carrier molecules do not change, the concentration gradient for exchanges falls. This Iranslocase is quite specific for cardiolipins (sec Table I; Ref. 284).

(iv) Two mitochondrial carriers of $\mathrm{Ca}^{2+}$. the uniporter for uptake and the $\mathrm{Ca}^{2+} / 2 \mathrm{Na}^{+}$antiporter for cgress, operate in senescent-heart mitochondria at rates $30 \%$ below those in mitochondria from hearts of young rats [258]. Some workers think that State 4 respiration involves $\mathrm{Ca}^{2} / \mathrm{Na}^{+} / \mathrm{H}^{+}$cycling, which might account for slowed State 4 iat $s$, but should extend to all substrates: no one has shown that $\mathrm{Ca}^{2+}$ normally regulate State 3 rates significantly.

In brain mitochondria oxidizing glutamatc/malate $\left(25^{\circ} \mathrm{C}\right.$ ). decreases in respiration (Table IV) are accompanied hy sharply diminished (by $68 \%$ ) glutamate uptake. probably involving the electroneutral glutamatc/ (OH antiporter [681]. The authors helieve that this may be a specific effect of senescence-induced membranc lipid changes which do not alter succinate and pyruvate oxidation rates - hut they did not measure succinate or pyruvate transport. With succinate as substratc. plots of $\lrcorner \psi$ vs. proton leakage rates calculated from State 4 respiration are similar in young and old; calculated $C_{M}{ }_{M}=$ are 1.37 and $1.22 \mathrm{ng}$ ion $\mathrm{H}^{+} \min ^{-1}$

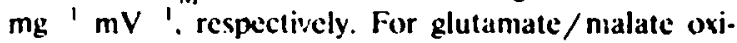
dation. $C_{M}{ }^{\prime \prime}$ corresponding values are 0.70 and 0.42 .

Altempts to delay senescence are interestingly related to phospholipid and EFA me'abolism. Repeated cold-induced hibernation, which starves the animal whilc decreasing caloric output, protongs the life of Tutkish hamsters by 50); [398]. Hibernation in ground squirrels increases heart membrane $18: 2$ content of tot.d lipids 3.4-fold $(x .5 \% \rightarrow 28.6 \%)$ at the expense of halved $16: 0$ and polyunsaturated fatty acyls contents [5:8]: no cardiolipin fatty acyl composition was presented hut becalese most heart $18: 2$ acyls are in cardislipins, involvement of cardiolipins seems likely. Caloric restriction (by - 4(1\%) ) extends life spal. . 40\% in laboratory rats [186.416.423.737.738]; 21 days of food restriction stimulates the J6-desaturase 3-fold and the 39 enzyme by $36 \%$; $[180]$. Re:cent publicity on antisenescence effects of growth hormone might be considered here in the light of studies showing that rat liver mitochondrial cardiolipin / phospholipid ratios vary directly with growth hormone levels (Table III; see section IV-C.2.d).

\section{IV-B.2. Tissues, species}

An early study of rat tissues [118] showed that cardiolipin/phospholipid ratios were $15-20 \%$ and cardiolipin $18: 2$ acyl contents are more than $75 \%$ of fatty 
acyl groups in heart and liver. less in skeletal muscle and splecn. and lowest in testis and brain (about $1-2 \ldots$ ), even though rat brain contains $0.6 \mu \mathrm{mol}$ of cardiolipins per $g$ wet weight [1(14). In all these cardiolipins, saturated fatty acyls contents were inverse to $18: 2$ acy! conients. Improved methods [612.720.729] confirm the differences in cardiolipin contents, hut reveal polyunsalurated fatty acyls instead of saturated fatty acyls in several tissues. Beef heart cardiolipins contain hardly any saturated fatty acyls [720]. Rat brain cardiolipins have saturated fatty acyl contents as luw as liver cardiolipins, little $18: 2$, and high contents of $20: 4(n-6)$ and $22: 6(n-3)$ polyunsaturated fatty acyls [729].

Intratissue heterogeneity has recently been described. Cardiolipins differ in mitochon'Iria of hepatocytes of the periportal and perivenous zones of rat liver lobules [79]. Percentages of $18: 2$ ac yis, monounsaturated and saturated acyls are, respectively, $75: 15: 5.4$ in periportal cardiolipins and $63: 20: 11$ in perivenous cardiolipins. Cardiolipin P contents per mitochondrial phosphulipid $P$ are $16.1 \%$ and $15.4 \%$.

Species: purified cardiolipins from beef hearts contain more than Y)\% 18:2 fatty acyl groups and about $7 \%$ other unsaturated acy!s $[6] 2,720]$. Beef heart mitochondria respire slowly in State 4: 6.7 and $26.6 \mathrm{ng}$ atom $0 \min ^{-1} \mathrm{mg}^{-1}$ (succinate and pyruvate/malate, respectively; $30^{\circ} \mathrm{C}$ [ [6]3]. Mituchondria from the hearts of chicks contain cardiolipins with $91 \%$ polyunsaturated fatty acyls and $9 \%$ saturated fatty acyls [537]: they respire in State 4 (pyruvate/malate, $30.5^{\circ} \mathrm{C}$ ) not at all in many cases, or at a rate of $3 \mathrm{ng}$ atom $0 \mathrm{~min}^{-1} \mathrm{mg}^{-1}$ while State 3 is 115-fold faster [6.59]. Thus, these highly unsaturated cardiolipins are associated with slow proIon leaks (low $\boldsymbol{P}_{11}$ ). but without corresponding measurements of $I p$ we can not he certain that this is duc to low $C_{M}$.

Rat skeletal muscle cardiolipins are heterogeneous. The amourts of cardiolipins (a measure of mitochondrial content) in white muscle, intermediate muscle. red muscle and heart muscle, are 1: $2.5: 5: 10$; mitochondrial cardiolipin/phospholipid ratios (which register membrane composition) are $1: 2.5: 2.5: 3.5$, respectively [476]. This accounts for the great range of cardiolipin/ phospholipid ratios in various studies, but $18: 2$ acyl contents are almost 80\% (Table III). (The mitochondrial electron transport and a-glycerophosphate dehydrogenase componints of rat skeletal musck fasttwitch and slow-twitch fibers are also heterogeneous in their response to exercise [523] and thyroid state [70, 706].) Cardiolipins of human muscle and pigeon breast muscle also have very high $18: 2$ acyl contents. Skeletal muscle cardiolipins of mice (the Arctic mouse Microius pennsylianicus and Bar Harbor strain C57BL/6J) have 20-50\% 18:2 acyls (Table III). The Bar Harbor mouse cardiolipins are repleted in 22: $6(x)$ -3) acyls (their muscle phosphatidylcholines and phosphatidylethanolamines even contain 2 to 3 -fold more $22: 6$ ] [5(13]. like heart muscle cardiolipins from rats fed diets containing fish oils. The fatty acids of a mouse laboratory chow include $4.3 \%(n-3)$-unsaturated fatty acids [4.31] which are abseitt from rat chows: 3 months on this diet doubles 22:6 acyl contents in liver and kidncy total phospholipids. Pig muscle cardiolipins have relatively low 18:2 acyl contents. slightly higher monounsaturated fatty acyls, and definitely higher saturated fatty acyls that include, uniquely, signiiicant amounts of 14:0,12:0 and 10:0 acyls (Table III). (Pig muscle phospholipids are all unusually saturated and lack ( $n-3)$-polyunsaturated acyls [116,127.369,485,585], probably because fed $18: 3(n-3)$ fatty acid competes poorly with fed $18: 2(n-6)$; these pigs are sensitive to the malignant hyperthermia syndrome, see section IV.C.2.c.) Fish (trout) skeletal muscle cardiolipins apparently lack $18: 2(n-6)$ and have fcw $18: 3(n-3)$ acyls but have high contents of saturated and 18: I fatly acyls (80\%) [224].

Hamster BAT mitochondrial cardiolipins are less unsaturated than rat BAT cardiolipins, and hamster BAT mitochondria have half the cardiolipin/phospholipid ratio (see section IV-C.2.c).

Plant cardiolipins are all in the inner membrane of mitochondria, where they comprise $15-25 \%$ of mitochondrial phospholipids $[43,44]$. Plant cardiolipins include $65-94 \% 18: 2 i n-6)+18: 3(n-3)$ fatty acyls, but no longer or more unsaturated fatty acyls (Table III). The unsaturation index under some conditions is more than 235, which is greater than in mitochondrial phosphatidylcholines and phosphatidylcthanolamines, and exceeds the unsaturation index of cardiolipins in animal mitochondria, except for those from heart and brain of rats fed fish oils. These high unsaturations may all denote phospholipid adaptations to low environmental temperatures. Plant lipid fatty acyl compositions are regulated by temperature and illumination [2(12], and the mitochondrial phospholipids adjust to the appropriate metabolic role. The unsaturation index in cardiolipins results mostly from the ratio of 18:2/18:3 acyls. Spinach petioles (lcaf stalks) do not perform photosynthesis; their mitochondria have high contents of respiratory chain components; the cardiolipins (together with phosphatidylcholines and phosphatidylcthanolamines) contain more $18: 2$ than $18: 3$ acyls [166] (Tahle III). In spinach leaves, that perform photosynthesis, cardiolipin $18: 3>18: 2$, and mitochondrial respiratory components are fewer. Cardiolipins from mung beans and their mitochondria also have more 18:3 than $18: 2$ acyls.

Cardiolipins from mitochondria of sycamore cells cultured at very low $(10 \mu \mathrm{M})\left[\mathrm{O}_{2}\right]$ contain $25 \% 18: 1$, $44 \% 18: 2$ and $24 \% 18: 3$ acyls $[43,44]$ (Table III); phosphatidyl-cholines, -ethanolamines, -inositols and -glycerols contain 55-80\% unsaturated fatty acyls: the 
necessary 19.112 - and 115 -desaturations must require little O.. When these cells grow in the presence of $250 \mu \mathrm{M} \mathrm{C}_{2}$, card.olipin / phospholipid ratios do not change, but in curuiolipins $18: 2(n-6)$ progressively replaces mo it of the 18:1 acyls, while $18: 3(n-3)$ remains constart; in mitochondrial phosphatidyl. cholines and phosphatidyicthanolamines, both $: 8: 2$ and $18: 3$ increase. The stoichiometric shift from 18:1 acyls to $18: 2(n-6)$ and $18: 3(n-3)$ acyls probably results from increased oxygen-dependent 112 - and 415-desaturations. However, saturated fatty acyl content of each phospholipid changes minimally. Growth in oxygen also fails to alter mitochondrial respiratory rates in State 4, State 3 and :tate 3d, or the thermotropic properties of States 3 and $3 u$. Tuerefore, State 4 proton leakage seems to correlate with the constant satura: al fatty acyl contents of cardiolipins (and other rhe spholipids) rather than the altered distribution: of unsaturated fatty acyls - which is consistent with th direct relationship between saturated fatty acyl contents in cardiolipins and State 4 proton leakage obscived in other mitochondria. These plant mitochondria oxidize very rapidly: Statc 4 respiration (succinatc, $25^{\circ} \mathrm{C}$ ) is $130 \mathrm{ng}$ atom $0 \mathrm{inin}^{-1} \mathrm{mg}^{-1}$ (cf) about 40 in rat liver mitochondria, see section IV. C.2.d), and State 3 four times faster; and the Arrhenius profile of uncoupled respiration remains inflected like that! of State 3.

However, comparisons with animal mitochondria may nut be valid. Plant (turnip and pea leaf) mito. chondria transport additional electrons through bypasses that do not pump protons, around complex 1 and the oxidase (see Ref. 442), which can contribute considerably to State 4 respiration. In State 4 (malate, $25^{\circ} \mathrm{C}$ ), pea mitochondria can be calculated to conduct at a $C_{\mathrm{M}^{\prime \prime}}$ of about $18 \mathrm{ng}$ ion $\mathrm{H}^{*} \min ^{-1} \mathrm{mg}^{-1} \mathrm{mV}^{-1}$. State 4 in turnip mitochondria is not regulated by carricr proteins: State 3 is regulated mostly by cytochromes $b c_{1}$ and $a a_{3}$, and less by the ATP-synthase [49)].

Chlorello itulgaris cardiolipin unsaturation is relatively low, and increases slightly after illumination, through accumulation of monounsaturated fatty acyls (Table 111). Tetrahymena, yyriformis mitochondrial cardiolipins contain few :.iturated fatty acyls (7\%) and mostly unsaturated fatty acyls $(83 \%)$, like heart cardiolipins; these cells must biosynthesize $18: 2(n-6)$ and $18: 3(n-6)$ from $18: 1$ fatty acyls by $\Delta 12$ - and $\Delta 6$-desaturations, as plants do. However, $T$. pyriformis seems to have no $\mathbf{4 1 5}$-desaturase, as judged from the ibsence of $(n-3)$-polyunsaturated fatty acyls in any of its phospholipids [337]; the fatty acyl profiles of the phosphatidylcholines + phosphatidylethanolamines $(50 \%$ of phospholipids) are similar to those of the cardiolipins. Acanthamoeba casteliani cardiolipins contain few 18:2 or $18: 3$ fatty acyls, $50 \% 18: 1$. and, uniquely, about
$30 \%$ of $2(i: 3(n-6)+20: 4(n-6)$ acyls [664] (Table III).

\section{IV.C. Experimental and patholegical rariation}

The fatty acyl composition of cardiolipins varies with (i) input of $18: 2(n-6)$, an essential fatty acid not synthesized by animal cells, in the diet or culture medium: (ii) input of fatty acids that affect $i: z$ metabolism of $18: 2$; and (iii) physiological or pathological eriterations in the metabolism of fatty acyls and phospholipids. Diets do not generally change cardiolipin, phospholipid ratios, altered metabolism does, both a 'ter cardiolipin fatty acyl compositions. Metabolic changes have been observed during ethanol-feeding. cofactor $\left(\mathrm{Mg}^{2+}\right)$ deficiency, environmental temperature variations, altered hormonal states, aging and development, tissue ischemia. cell transformation and in some specific disease states, as is discussed below. Obviously, mitochondrial fatty acyl composition can be more precisely and extensively manipulated, either through fally acyl supply or induced metabolic changes, in cultured acrobic cells than in living animals. This advantage must be weighed against the uncertainty of equating viability of cell cultures with normal cell function and compatibility in whole organisms. For this reason, insights from whole animal experiments seem valuable in interpreting events in cclt suspensions. Some chaso... $\vdots-\bar{c}$ sardiclipin fatty acyl unsaturation and in cardiolipin, phospholipid ratios are associated with altered mitochondrial inner membrane proton permeability (State 4 respiration) and ADP phosphorylation (State 3).

\section{IV.C.I. Dietany manipulation. whole animal}

IV-C.1.a. Essential fatty acid $(18: 2(n-6))$. deficiency. Dietary requirements of $18: 2$ or other ( $n-$ 6)-polyunsaturated fatty acyls are probably in the range of $1 \%$ of total calories [7] but vary with the clinical or metabolic criterion usec' for physiological normality $[546,547]$. Varied dietary inpit of hydrogenated or $18: 2$ fatty acids does not change vardiolipin/phospholipid ratios in liver [41,658]. heart [41,45,90,368 548,729], skelctal muscle [41] and intestinal mucosa [740].

Several workers have noted that 'homeostatic' mechanisms keep mitochondrial total lipid and pnospholipid unsaturation/saturation ratios constant when diets contain as few 18:2 acyls as $9 \%$ of total fatty acyls, and fat contents are $4-16 \%$ [105.211]. It should be noted that rat liver mitochondria normally contain about $20 \%$ of cardiolipin / phosphulipid and the cardiolipins contain some $60 \% 18: 2$ per total fatt, acyls (Täble III) or up $1080 \%$ [716]; the other phospholipids contain only $9 \% 18: 2$ but are $80 \%$ of total phospholipids. Ergo, two thirds of the 18:2 acyls in total phospholipids of liver mitochondria are in cardiolipins. 
Depletion of total phospholipids 18:2 acyis by EFA deficiencies thus reflects mostly the cardiolipin compartment. However, examination of incividual phospholipids is still necessary to determine the extent of dietary or metabolic effects, and opposing changes in 18:2 acyl contents of cardiolipins and the other phospholipids can make measurements of $18: 2$ contents of total phospholipids misleading (see scctions IV-C.2.a and $\mathrm{c}$.

Diets low enough in ( $n-6)$-unsat!rated fatty acyls evoke compensatory metabolic mechanisms. First. rapidly activated and induced hepatic enzymes of de novo biosynthesis and 19-desaturation produce saturated fatty acyls-CoA and monounsaturated fatty acyls: the moncunsaturated fatty acyls replace 18:2 acyls in cardiolipins but not in other phospholipids (716). Diets that contain lipids with only hydrogenated fatty acids also displace some 18:2 acyls with endugenous or exogenous monounsaturated fatty acyls, in the pattern seen with fat-free feeding. After a few wecks, increased hepatic elongations and 56 - and 45 -desaturases [58] convert endogenous and exogenous monounsaturated fatty acyls-CoA $\rightarrow 2(1 \cdot 3(n-9)-C o A$, and some $20: 3(n-9)$ acyls are... rerified into phosphatidylcholines and phosphatic. " thanolamines but not cardiolipins.

Diets that are fat-free, or that contain hydrogenated fats, replace nos! of the $: 8: 2$ acyls in rat liver, heart, kidney and intestinal mucosa cardiolipins with monounsaturated fatty acyls (Table III). They raise monounsaturated fatty acyl (including trans-monounsaturated fatty acyls found in hydrogenated fats) proportions in rat liver cardiolipins from 10-20\% of total fatty acyis in controls to as much as $70 \%$. A fat-free diet removes all 18:2 and most 20:4 acyls from rat brain cardiolipins, which normally contain only $13 \%$ $18: 2$, and slightly raises contents of saturated fatty acyls, monounsaturated fatty acyls, and a $20: 3$ acyl [41] (Table III).

Fatty acyl composition of phospholipids has been measured as early is 1 day after the start of an EFAdeficient dict. but for some reason oxidative phosphorylation has been examined after at least 4 weeks and mostly after 8 weeks. Change; in mitochondrial morphology and enzyme activities appear as early as $1-2$ weeks and make mitochondria the most sensitive indicator of EFA-depletion [7,265]. Hayashida and Portman [265] found that 1 week of a fat-free- or saturated fatty acyls-diet depresses liver mitochondrial total lipid $18: 2$ content from $23 \%$ (control) to $7 \%$, and begins to release succinate dehydrogenase activity from its partly crypticized, membrane-bound state.

EFA-deficiency linearly depletes $18: 2(n-6)$ acyl groups in cardiolipins of rat liver mitochondria, from 79\% of total fatty acyls to less than half in 7 days and to a quarter in 66 days [716], see Table V.
TMBIF V

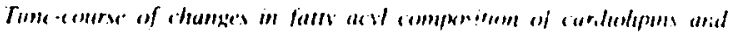

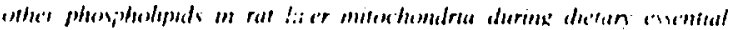

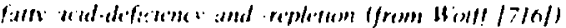

\begin{tabular}{|c|c|c|c|c|c|}
\hline \multirow{3}{*}{$\begin{array}{l}\text { Fatly } \\
\text { acyl }\end{array}$} & \multicolumn{5}{|c|}{ 'i of total fatts acyls in cardiolipins } \\
\hline & \multicolumn{3}{|c|}{$\overline{\text { fat-ficc dict }}$} & \multicolumn{2}{|c|}{$+1 F A$ diet" } \\
\hline & day 0 & day 2 & diay 7 & das $x$ & Jay 12 \\
\hline Ih:0 & 3 & 4 & 9 & 3 & 3 \\
\hline $16: 1(\therefore,-7)$ & 1 & $x$ & is & 13 & 7 \\
\hline $18: 0$ & $\mathbf{I}$ & 1 & 1 & 1 & 1 \\
\hline$(8:(1)-4)$ & 4 & 12 & IX & 4 & 4 \\
\hline $18: 1(n-7)$ is & $x$ & 1.3 & 18 & 15 & 14 \\
\hline $18: 2(n-6)$ & 74 & 57 & $3.3 \mathrm{n}$ & 47 & 62 \\
\hline Other $(n-6)$ & 4 & 5 & 5 & 5 & 4 \\
\hline $20: 3(n-6)$ & 0 & $i$ & is & $i$ & 1) \\
\hline $22:(x(n-3)$ & 1 & 1 & 1 & 1 & 1 \\
\hline
\end{tabular}

$r$ of total fatty acyls in other phospholipids

\begin{tabular}{lrrrrr}
\hline $16: 1)$ & 24 & 26 & 25 & 22 & 23 \\
$16: 1(n-7)$ & 1 & 3 & 3 & 1 & 1 \\
$18: 0$ & 22 & 16 & 16 & 23 & 21 \\
$18: 1(n-4)$ & 4 & 8 & 17 & 14 & 7 \\
$18: 1(n-7)(i s$ & 2 & 6 & 5 & 2 & 2 \\
$18: 2(n-6)$ & 4 & 3 & 2 & 8 & 10 \\
Oiher $(n-6)$ & 21 & 16 & 17 & 23 & 25 \\
$20: 3(n-9)$ & 11 & 3 & 4 & 1 & 11 \\
$22:(x)-3)$ & 16 & 8 & 7 & 11 & 10
\end{tabular}

- +EFA = 4r; fat in diet, containing 50r; $18: 2(n-6)$ and $3.7 \%$ $18: 3(n-3)$ falty acids:

$b+$ approx. 4'i $1 K: 2(n-7)\{721\}$

- identified hy $R$ !.. Wolff [716]: in oh days on the fat-ifee dict.

" $0.8 \%$ " 2(1):3(n-9) acyls appear in "Ls, and

:3C; $20: 3(n-9)$, in other PLs.

The 18: 2 content in cardiolipins and in other mitochondrial phospholipids (where 18:2 acyls are unly $9 \%$ of total fatty acyls) starts to fall in 2 days. when $18: 2$ acyls in cardiolipins become $73 \%$ of the $18: 2 /$ total phospholipids. Given a cardiolipin/phospholipid ratio of $12.7 \%$ (Table III), calculated $18: 2$ content of total

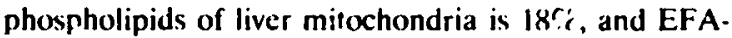
deficiency depresses it to $10 \%$ on day $2.6 \%$ on day 7 . and $4 \%$ on day 66 (Table V): a fat-free diet replaces 18:2 acyls in total phospholipids with monounsaturated fatty acyls even faste in rats prepared metabolically by fasting (sec helow). Resupply of EFA raises 18:2 acyl contents in cardiolipins by $50 \%$ in 1 day. EFA-deficiency-induced monounsaturated fatty acyls $(16: 1(n-7), 18: 1(n-9)$, and cis-18: $1(n-7))$ rapicily and linearly replace $18: 2$ acyls, but only in cardiolipins, to reach $54 \%$ of total fatty acyls. Repletion with EFA replaces some cardiolipin monounsaturated fatty acyls rather slowly. and cis-18: $1(n-7)$ acyls persist tenaciously. Saturated fatty acyls, other $(n-6)$-polyunsaturated fatty acyls, and $22: 6(n-3)$ acyls are normally minimal in cardiolipins and major components in other phospholipids; EFA-deficiency or -feeding does not affect these acyls in either locus. Unexpec' edly, the 
20: $3(n-6)$ fatty acyl group appears in liver (and kidncyl cardiolipins within 2 days of EFA-deficiency. This desaluration derivative of the deficicnt dietary $18: 2$ fatty acid strongly hinds cardiolipins [716] and may accumulate progressively through [:-A deficiency-induced J6-desaturations [58]: $2(1): 3(n-6)$ boosynthesis should further deplete hepatic 18:2 acyis. The diagnostic fatty acid of prolonged EFA-citicier y. 20:3(n -9). appears in cardiolipins only at 9 weeks, but accumulates mucil carlier in other pirospho'ipids (4\%; in 7 days, $13-21 \%$ in 9 weeks $[265.710]$, where it is also quickly replaced when EFA are ied. These findings confirm a central role of rat liver mituchondria! cardiulipins in EFA metabolism, and leav: the altered fatty acyl composition of cardiolipins to acceunt for observations that EFA-deficiency increases proton leakage.

EFA-deficient diets increase State 4 respiration and proton le:akage; see Ref. 284 for a critioue of apparently contrary findings. In recent sturdics, saturated fatty acyl-dicts accelerate rat liver mitochondrial State 4 respiration $\left(25^{\circ} \mathrm{C}\right)$ by $25-50 \%$ in $6-14$ weeks $[147,525]$. By the criteria of the State 3: State 4 ratio sather thin the absolute rates, together with the ADP/O ratio. Rafael et al. [525] and some other workers (sec Ref. 284) conclude that EFA-deficiency has no effect on oxidative phosphorylation. State 3 respiration at $25^{\circ} \mathrm{C}$ increases $27 \%$ [525] or not at all [147], or $37 \%$ at $37^{\circ} \mathrm{C}$ (glutamate) where the $P / O$ ratio decreases significantly at 9 weeks $[153,154]$. The BMR rises $25 \%$ [525] or $16 \%[736]$, in units of rate of $\mathrm{O}_{2}$ consumption per (kg body weight) ${ }^{0.74}$, the appropriate power term [361]. The accelerated mitochondrial respiration accounts for the $t^{\prime}$ le-mogenesis (see section IV-C.2.c); others disagree [525?

The me nbrane potential under State 4 conditions (succinate) 1 mitochondria from control rats is 186 $\mathrm{mV}$, from ECA-deficient rats. $187 \mathrm{mV}$ [52.5], and the effective proton conciuctance can be calculated to be 0.66 and $0.90 \mathrm{ng}$ ion $\mathrm{H}^{+} \mathrm{min}^{-1} \mathrm{mg}^{-1} \mathrm{mV}^{-1}$, respectively. An increased State 4 proton leak rate with unchanged $A p$ means that EFA-deficiency increascs $C_{\mathrm{M}^{\prime \prime}}$, the effective proton conductance of the nner membrane, in rat liver mitochonuria. Presumably, EFA-deficiency exerts its effects via the altered fatty acyl compcsitions, which are most extreme in the cardiolipins, and most compensated in the phosphatidylcholines and phosphatidylethanolamines, of mitochondrial inner membranes. The simplest mechanism is an increase in the normal proton phospholipid-leak, but abnormal mechanisms might involve altered phospholipid-protein interactions that introduce an $F_{0}$-leak or $\mathrm{X}$-leaks, or increased $\mathrm{H}^{+}$/O-slip (see section II-A).

Hamster liver mitochondrial cardiolipins are even more highly unsaturated than rat cardiolipins $(85 \%$ $18: 2+11 \% 18: 1$ acyls) and have almost no saturated fatty acyls, like beef heart cardiolipins (Table III).
EFA-deficiency brings it: 2 content down to $30 \%$ and substitutes 60\%? monounsaturated fatty acyls plus $1.4 \%$ 20:3 acyls: I find no studies on liver mitochondrial respiration in EFA-deficient hamsters.

Based on sludies of EFA-deficient rats. Divakaran and Venkataraman [1.54] originally proposed that the flinction of cardiolipins in the liver mitochondrial membranc differs from that of the other phospholipids. From this review of later findings. the great changes in cardioliniss might be responsible for increased proton leakage and State 3 respiration. The hulk phosphatidylcholines and phosphatidylethanolamines would affect bulk physical propertics like the effects of iemperature on rate processes and membrane fluidity, although phosphatidylcholine and phosphatidylethanolamine vesicles leak protons (see section II-A); their repletion with $20: 3(n-9)$ fatty acyl might account for normal propertics measured in EFA-deficiency.

Either a hydrogenated-fat-diet, or T4-treatment $(0.1$ $\mu \mathrm{g} \mathrm{g}{ }^{\prime \prime} \mathrm{d}$ ' for $?$ days) of EFA-sufficient rats, accelerates liver mitochondrial State 4 respiration at $25^{\circ} \mathrm{C}$. T4 given to EFA-deficient rats accelerates State 4 rates (to State 3u levels) and linearizes Arrhenius profiles [147]. Hyperthyroidism depletes $18: 2$ acyls severely in cardiolipins and less in other phospholipids, but raises cardiolipin saturated fatty acyls and $(n-6)$-polyunsatilrated fatty acyls contents rather that monounsaturated fatty acyls (sec section IV-C.2.d), it would be interesiing to see the fatty acyl composition of liver cardiolipins from EFA-deficient hyperthyroid rats.

Feeding rats starved 1-2 days a fat-free diet (i.e. carbohydrates and proteins) produces the acutest EFA-deficiency, probably by first introduciing me:abolic changes that accentuate and hasten changes due to absence of 18:2 fatty acid input. Starvation signals glucagon and catecholamines, and protein phosphorylation. Subsequent fat-free feeding signals insulin release and dephosphorylation of the extensively phosphorylated proteins, which stimulates hepatic fatty acyl synthesis [311,650] and 19-desaturations [317] within minutes or hours. Fatty acid synthetase activity rises 10 -fold in $12 \mathrm{~h}$. 50)-fold in $48 \mathrm{~h}$ : newly synthesized enzyme appears in $12 \mathrm{~h}$ and increases less markedly [192]. Within a few hours the monounsaturated fatty acyls begin to replace $18: 2$ acyls in total phospholipids of rat liver mitochondria and $18: 2$ content falls from $23 \%$ to reach $5.3 \%$ in 48 h [9]; a simple fat-free diet acts more slowly. Neither fatty acyl contents of cardiolipins nor oxidative phosphorylation have been reported under these conditions, to my knowledge. However, from Wolffs study [716] it seems likely that changes in fatty acyl/cardiolipins predominate, except for the (late) appearance of $20: 3(n-9)$ acyls in the uther phosphoiipids.

In humans, total parenteral feeding with fat-free fluids that contain glucose suppresses adipose tissue 
lipolysis and rakcs $18: 2(n-6)$ acyls stored in triacylglycerols unavailahle: biochemical signs of EFA-deficiency eppear within 1 weck in infants. with their extra demands for growth (sec Ref. 546).

A fat-free dict given for 14 weeks depletes rat heart cardiolipins of $18: 2$ acyls, substitutes monounsaturated fatty acyls $(63 \%)$ and $(n-6)$-polyunsaturated fatty acyls $(13 \%)$ synthesized in the liver. and maintains a cardiolipin free of saturated fatty acyls [41]. Cardiolipins are the most resistant among cardiac phospholipids against changing fatty acyl contents when rats are fed diets high in saturated fatty acyls [368]. Feeding hydrogenated oils introduces $6-39 \%$ trans $18: 1(n-9)$ into heart mitochondrial phosphulipids [308]; trans-monounsaturated fatty acyls are structurally like saturated fatty acyls [716].

Cardiolipins in heart mitochondria from rats so fed for 6 weeks contain somewhat less 18:2 than corn oil controls ( $40 \%$ vs. $72 \%$ ); their saturated fatty acyls are only slightly elevated (11\% vs. $6 \%)$, their monounsaturated fatty acyls are more markedly higher $(16: 1+$ $18: 1$ are $27 \%$ vs. $13 \%)$ and trans-18: $1(n-9)$ appears (17\%) [308]. These cardiolipin changes are less than in liver mitochondria but are still accompanicd by $15-30 \%$ rises in State 4 respiration $\left(37^{\circ} \mathrm{C}\right.$, pyruvate or $18: 1$ carnitine, but not glutamate) and no decrease in phosphorylation efficiency.

Fed linelaidic acid (trans, trans-18:2(n-6)) appears in rat heart mitochondrial phosphatidylethanolamines $(5.5 \%)$ and phosphatidylcholines $(4.5 \%)$, but not in cardiolipins [115] (Table III). State 4 respiration (succinate or glutamate / malate, $25^{\circ} \mathrm{C}$ ) remains unchanged while State 3 (and thereby the respiratory control ratio) decreases (see section 11-B). These findings are consistent with the fatty acyls of cardiolipins regulating the proton leak; the altered mitochondrial phosphatidylcholines and phospliatidyicthanolamines leave the leak unchanged, but may be involved in down-regulating State 3 respiration by changing $C_{i}$ values of the rat:limiting transporter proteins.

It is striking that these dietarily-induced EFA-deficiencies do not compensatorily increase the trace percentage contents of $20.3(n-9)$ fatty acyls in liver. heart and kidney cardiolipins. The 20:3 acyls partly displace $20: 4$ acyls in all the other phospholipid fractions (phosphatidyl-choline, -ethanolamine, -inositol and -serine), more in liver phospholipids than in renal phospholipids [580]. EFA-deficiency produces loosecoupling of liver mitochondrial oxidative phosphorylation despite the maintenance of relatively high total phospholipid unsaturation, perhaps because the low $18: 2$ and high $16: 1+18: 1$ contents in the cardiolipins persist - given the special relationship between cardiolipins and proton conductance adduced here.

IV.C.l.b. Feeding $(n-3)$-unsaturated fatty acids. Linolenic acid, 18: $3(n-3)$, is biosynthetically essential because vertebrates lack a 115 -desaturase $[\$ 8-60)]$. At adequate levels of $(n-6)$-polyunsaturated fatty acyls. (n - 3)-polyunsaturated fatty acyls are satisfactory substitutes in vertehrat:s: arctic marine anirals, and humans that subsist wa them, as well ä dogs fed fish oils [126], have high $(n \cdot \therefore$-nolyunsaturated fatty acyls levels and relatively low ( $n$ - $6:$ livels in membrane phospholipids [547.608] but exhibit no classical signs of $(n-6)$-EFA-deficiency. Fed $(n-3)$-unsaturated acids readily replace a part of the $(n-6)$-unsaturated acyls in heare phospholipids and neutral lipids [2.36], and in-3)-acylCoA molecules are preferred substrates for desaturases [117]. Tissue $(n-3)$-polyunsaturated fatty acyls content is biologically conserved to such an extraordinary degree that two generations of rats must bc fed a purified $18: 3(n-3)$-deficient diet to deplcie the (n - 3) polyunsaturated fatty acyls, especially 22:6 acyls, in brain, heart, muscle, retina and liver [657]: $22:(x-3)$ acyls ure replaced mainly by $22: 5(n-6)$ acyls - successfully, since the animals survived. The only signs of verified $18: 3(n-3)$ deficiency in lumans are neurological ahnormalities [294] which may be connected with the high percentage of $22: 6(n-3)$ acyls in brain cardiolipins (Table III).

Fecding diets supplemented with fish oils partly replaces cardiac 18:2 acyls with the fed ( $n-3)$-polyunsaturated fatty acyls and derivatives. In rats fed a diet containing $10 \%$ cod liver oil for more than 3 months, heart cardiulipin / phospholipid ratio rises from $7.8 \%$ (control) to $12.4 \%$, and the cardiolipins are not depleted of 18:2 acyls but the chosphatidylcholines and phosphatidylethanolamines are [234]. On the other hand, sardine or tuna [90] oil in diets does not change cardiolipin/phospholipid proportions. Heart mitochondria of ra's fed sardine oil for 10 days contain cardiolipins ve,y low in $18: 2(n-6)$ acyls, as in EFAdeficient rats; fed or derived $(\boldsymbol{n}-3)$-polyunsaturated fatty acyls, especially $22: 6(n-3)$, rather than saturated fatty acyls (which appear in amounts of $9 \%$ of total fatty acyls) or monounsaturated fatty acyls, replace cardiolipin 18:2 acyls, raising the unsaturation index to 316 [729]. No Mead acid, 20: $\boldsymbol{Y}(\boldsymbol{n}-9)$, appcars in the cardiolipins, or (not shown) in the phosphatidylcholines and phosphatidylethanolamines, although they too are depleted in $18: 2(n-6)$ and $20: 4(n-6)$ fatty acyls. It is striking that, in the face of the $(n-6)$-polyunsaturated fatty acyl depletions, the proton leakage rate in the heart mitochondria from sardine oil-fed rats, as expressed by State 4 respiration (succinate or glutamate $/$ malaie, $25^{\circ} \mathrm{C}$ ), is unchanged or decieases.

It should be noted that the total fatty acyls in 'fish oil-diets' contain up to $13 \% 18: 2(n-6)$ fatty acid $[90,126,729]$, either because they supplement an ordinary chow or because the total fatty acyls of sardine oil, for example, include $6 \% 18: 2$ acid, enough to avoid absolute deficiency. Actual substitution of $18: 3(n-3)$ 
fatty acid for $18: 2$ in an otherwise fat-free diet (fod for 12 weeks) strips ra! liver cardiolipins of just about all $18: 2$ acyls, elcvates monounsaturated fatty acyls, $18: 3(n-3)$ and $22: 6(n-3)$ contents in cardiolipins (Table III), and cvokes the appearance of the $20: 3(n$ -9) acyls typical of EFA-deficiency in all the phosphatides except cardiolipins, but does not change cardiolipin/phospholipid ratios [658]. Such rats rapidly develop clinical signs of EFA-deficiency, but oxidative phosphorylation does not scem to have been measured in their liver mitochondria.

Pigs have been fed diets containing $8 \%$ fat, which in controls contained $14 \%$ of $18: 1(n-9), 55 \%$ of $18: 2(n$ $-6)$ and $6 \%$ of $18: 3(n-3)$ per total fatty acids [485]. Diets enriched in $18: 3(n-3)$ (progressively up to $31 \%)$, high in $18: 1(31 \%)$ and low in $18: 2(n-6)$ $(19 \%)$ introduce $18: 3(n-3), 20: 3(n-3)$, and $20: 5(n$ -3) acyls that are not found in renal cardiolipins of pigs on the control diet. These $(n-3)$-unsaturated acyls displace some 18:2 and 18:1 acyls [585] (Table III). The high-18:3 diets lead to impaired feed utilization for weight gain (mitochondrial dysfunction was mentioned), enlarged heart, liver anci kidneys, anemia, and 'yellow fat disazice that may arise from deposits containing polyunsaturated fatty acyl percxidation products. These toxic effects werc attributed in part to the known competitiveness of $18: 3(n-3)-\mathrm{CoA}$ for 46-desaturase. Diets enriched in $18: 1(n-9)$ fatty acid (to $43 \%)$ and constant in $18: 2(n-6)(19 \%$, a low value) and $18: 3(n-3)(20 \%$, high) raise pig kidney cardiolipin 18:1 acyl content only slightly, deplete $18: 2$ acyls somewhat, and substitute about $20 \%$ of $18: 3(n-$ $3)+20: 3(n-3)$ acyls.

IV-C.I.c. Feeding erucate. Erucate-feeding tests how fatty acyl composition of cardiolipins affects cardiac oxidative phosphorylation, because rats incorporate fed $22: 1(n-9)$ fatty acid into heart $[45,308,537,735]$ and liver [735] mitochondrial cardiolipins but not into phosphatidylcholines or phosphatidylethanolamines, without altering cardiolipin/phospholipid ratios, and exhibit in vivo and morphologica: signs of myocardial damage. In contrast, feeding hydrogenated fats changes fatty acyl in heart cardiolipins least, see (a) above. In heart mitochondrial cardiolipins from rats fed erucate diets for $\geq 28$ days (but not less), 18:2 drops to $40-55 \%$ (cf. $68 \%$ in rats fed corn oil diets), $4 \%-8.5 \%$ $22: 1+20: 1$ acyls appear, saturated fatty acyls increase to $10-25 \%$ (6\% in controls) and monounsaturated fatty acyls to $27 \%$ (13\% in controls) tc ieplace the 18:2 acyls (Table III). These heart mitochondria respire faster in State $4\left(37^{\circ} \mathrm{C}\right.$, with glutamate or 18:1carnitine) and slower in State 3 [308]. Compared with soybean-oil-fed control rats, $22: 1$-feeding for a: least 28 days accelerates State 4 respiration $60 \%-100 \%$ with several substrates [105]. In contrast, in heart mitochondrial cardiolipins from chicks fed 22:1 fatty acid for 24 days, the decreased $18: 2$ acyls $(77 \% \rightarrow 47 \%)$ are replaced by $22: 1(11 \%)$ and $20: 1(n-11)$ acyls $(17 \%)$, with no change in $16: 0$ and no appearance of $18: 0$, $10: 1$ or i8: 1 acyis (that are absent from safflower-oilfed controls) [537]. Here State 4 respiration remains I'nchanged (pyruvate/malate, $37^{\circ} \mathrm{C}$ ) while State 3 and the $A D P / O$ ratios decline slightly. These findings suggest again that the substituting saturated fatty acyls and/or monounsaturated fatty acyls, rather than the decrease in cardiolipin 18:2 acyls, correlate with proton lcakage. Findings that dietary $22: 1$ fatty acid fails to alter respiratory rates or phosphorylation in heart mitochondria [156] were obtained in rats fed 3 days (sic), were not accompanied by fatty acyl analyses of the phospholipids. and are to be expected from the studies cited above.

Observations that accumulated saturated fatty acyls and monounsaturated fatty acyls in cardiolipins obtained from EFA-deficient correlate with increased proton leakage seem to be contradicted by the effects of unsatrirated fatiy acyls-deficiency in yeasts (see seciiun IV-C.4), where monounsaturated fatty acyls decrease proton leakage. and substituted saturated fatty acyls increase it. But the cardiolipins of the yeasts studied contain $96 \%$ monounsaturated fatty acyls and no $18: 2$ acyls. If the differences between rat and yeast mitochondria do not preclude a comparison, monounsaturated fatty acyls make yeast mitochondria more leaky to protons than do $18: 2$ acyls in rat mitochondria.

If, as much of the evidence cited above suggests, the fatty acyl composition of cardiolipins is connected with mitochondrial proton leakage, the thermotropic properties of State 4 respiration should change with fatty acyl substitutions, especially saturated fatty acyls and monounsaturated fatty acyls for polyunsaturated fatty acyls. Surprisingly little attention appears to have been given to the measurement of Arrhenius profiles of rate processes in oxidative phosphorylation in the long. known states of EFA-deficiency. These would be important in determining mechanisms, and because altered profiles make the temperature of assay crucial for demonstrating rate changes $[98,99]$.

\section{IV.C.2. Metabolic manipulation, whole animal}

IV-C.2.a. Feeding ethanol. Ethanol-feeding raises 18:2 and depresses 20:4 acyl proportions in rat liver mitochondrial total phospholipids [194,430], which has been ascribed to an accompanying decrease in activities of microsomal fatty acyl-CoA 45 - and 46 -desaturases [458]. A similar fatty acyl redistribution, plus an accumulation of $22: 6(n-3)$ acyls, occurs in rat liver microsome phospholipids, and because the activities of 49- and 45-desaturases are increased and that of the 16-desaturase remains normal, the effects of desaturases on membrane fatty acyl changes were dis- 
counted [672]. 30 days of feeding ethanol as $36 \%$ of tota! calories augments $18: 2$ and depletes $20: 4$ in phosphatidylcholines and phosphatidylethanolamines [128] but ethanol as $14 \%$ of calories leaves phosphatidylcholines and phosphatidylethanolamines unchanged [686]. However, in cardiolipins ethanol-feeding depletes $18: 2$ content by $17-25 \%[128,686$ ? and reciprocally increases saturatcd fatty acyls and $18: 1$ acyls (Table 1II), which does not seem attributable to any accumulation of 18:2-CoA molecules available to mitochondria. Cardiolipins of periportal and perivenous zone mitochondria contain less $18: 2$ and more monounsaturated and saturated acyls than controls (see section IV-B.2); the percentages in periportals are $47: 31: 16$ and in perivenous, $38: 36: 22$; and cardiolipin $P$ per phospholipid $P$ ratios are 16.6 and 17.2 [79]. Overall mitochondrial cardiolipin/phospholipid ratios decrease minimally in rat livers $(7 \%)[128,168$, 574,654 ] and baboon livers (3\%) [17]. but may increase in mouse livers [430]. By measurements of total cardiolipins, ethanol-feeding does not produce very large differences from control cardiolipins yet hepatic 'ethanol-cardiolipins' exert some specific effects on membrane function. The zonal heterogeneity of these cardiolipins implies that the perivenous hepatocyte fraction, which is more abnormal than the mean value, may be the most effective; studies on its power to make mitochondria tolerant to ethanol (see below) should be informative.

Ethanol-feeding (i) depresses liver mitochondrial respiration and changes its Arrhenius prnfiles in a way that is seen when inner mernbrane phospholipid fatty acyl compositions are altered; and (ii) induces adaptations of membrane structure stability against ethanol applied in vitro that are specifically due to altered anionic phospholipids - in mitochondria, the cardiulipins.

(i) Rat liver mitochondrial respiration is altered by prolonged feeding of ethanol as fcllows. In six stidies using succinate as substrate at $30^{\circ} \mathrm{C}$, State 4 rates decrease by an average of $16 \%$, State 3 by $34 \%$ $[81,304,562,574,622,623]$. Of course the greater depression of State 3 respiration decreases the mean respiratory control ratio, but this does not signify 'ioose-coupling', a term that describes decreased control ratios caused by protonophorically increased Siate 4 respiration. State 4 and $3 \mathrm{u}$ respiration rates (NADH, $25^{\circ} \mathrm{C}$ ) are also slowed in inner membrane paricles [651]. State 4 respiration (malate / glutamate, $25^{\circ} \mathrm{C}$ ) in intact mitochondria decelerates while $\Delta p$ stays at control levels and State 3 decreases even more [558]. Changed Arrhenius profiles of rates in both respiratory states diminish the ethanol effect at higher temperatures of measurement [555,557]. An opposing view, based on the lack of changes in fatty acyl composition of mitochondrial total phospholipids [219], has been refuted by pointing out that the fatty acyl changes in the relatively small cardiclipin fraction are obscured when total phospholipids are measured [560]. Several analogous instances are pointed out in this review.

The mechanisms that retard State 4 and State 3 respiration must differ. Slowed State 4 indicates a slower transmembrane proton-leak; the constant $S p$ indicates that ethanol-feeding diminishes $C_{\mathrm{M}^{\mathrm{H}}}$. Altered $C_{\mathrm{i}}$ values appear responsible for the slowed State 3 rate. Although Spach and Cunningham [622] do not present a complete profile of control coefficients, their titrations with specific inhibitors indicate that cy. tochrome $a a_{3}$ limits State 3 respiration more than in control mitochondria $\left(C_{i}=0.17\right)$ when dicarboxylate substrates are oxidized. Although ethanol-feeding results in lower mitochondrial general synthesis of proteins, and in depressed content and activity of cytochrome $\mathrm{aa}_{3}[17,114,562,651]$, the amount of $\mathrm{cy}$ tochrome oxidase apoprotein does not decrease, but only half of it is in active form with bound heme-a $a_{3}$ [652]. The mechanism of this ethanol effect is not known; Thayer and Rubin [652] suggest that altered membrane lipids may interfere with heme-protein assembly, or that holoenzyme is degraded. The specific association of cytochrome oxidase and cardiolipins is well known (see section II-B). These authors also note that other mitochondrial components limit State 3 respiration, especially the ATP-synthase and the NADH dehydrogenase. Netther of these regulates normally in liver mitochondria [228], but decreased activities of mitochondrial ATPase and succinate dehydrogenase are reported [562,651]. Effects of ethanol on the major regulators of normal rat liver mitochondrial State 3 respiration, the carriers of ADP/ATP and dicarboxylate substrates, do not seem to have been studied. Chronic ethanol-feeding of rats produces a partially 'hyperthyroid' metabolic pattern in liver $[322,323]$ : decreased glvcogen content; increased $\beta$-adrenergic sensitivity; increased microsomal glucose-6-phosphatase, NADPH: cytochrome $c$ reductase. NADH oxidase, and $\alpha$-glycerophosphate dehydrogenase activities; increased mitochondrial size; and accelerations of respiration by $30 \%$ (at $37^{\circ} \mathrm{C}$ ) in liver slices [677] and $23 \%$ in perfused livers [322] of ethanolized rats. Propylthiouracil, in dosage that corrects hyperthyroidism in human suhjerts, is said to protect their livers against the lethal toxic effects of chronic excessive ethanol intake [483]. However, these findings are not accompanied by characteristic 'hyperthyroid' increases in cytochrome oxidase amounts and activity in isolated mitochondria, or the typical changes in liver phospholipid fatty acyl profiles, especially in unresolved cardiolipins, and seem paradoxical in view of the decreased mitochondrial respiration. Studies on periportal and perivenous mitochondria might settle these inconsistencies; cardiolipins from mitochondria of perivenous 
hepatocytes of ethanol-fed rats show an ahnormal fatty acyl pattern (see above and Ref. 79) almost as extremc as that in hyperthyroids (Table III). The apparent paradox may also be explained by observations that ouabain returns the high respiratory rates of fiver slices to control levels [677]: the hypernetabolic effects of ethanol may reflect in part the plasma membrane ouabain-sensitive NaK-ATPase, not mitochondria. That ATPase activity shows altered lipid-dependency [304] but the nature of the membrane lipid changes is not yet clear. Plasma membranc anionic lipids include phosphatidylinosituls (6.7\% of total phospholipids) and phosphatidylserines (4.7\%), and ethanol feeding does change their properties in conferring membrane adaptation.

(ii) Continued administration of ethanol blunts in vitro responses to ethanol, in liver mitochondria through changes in cardiolipinc. Ethanol, 0.5-1.0 M (150-3(X) $\mu \mathrm{mol} / \mathrm{mg}$ mitochondrial protein) produces real loose-coupling (State 4 increases by $63^{\circ} \%$ at $25^{\circ} \mathrm{C}$ and $80 \%$ at $40^{\circ} \mathrm{C}$ much less eflectively in liver mitochondria from ethanolized rats than in those from normals: a 'tolerance' [559]. Membranes prepared from mouse brain synaptosomes, rat ersthrocyies, liver mitochondria or microsomes, or pancreatic acini, adapt to the continued presence of ethanol. Fed ethanol produces blood levels around $50 \mathrm{mM}$ in 4-5 weeks, when in vitro exposure to [ethanol] $=50-100 \mathrm{mM}$ (about 20 ) $\mu \mathrm{mol} / \mathrm{mg}$ ) disorders mcmbrane structure little or not at all, although it disturbs bilayer structure in $\mathrm{mem}$ branes from sober controls. Ethanol-fecding itself does not consistently increase the order of the fatty acyl chains. Membrane tolerance resides in the phospholipids of the membrane and not in the proteins, and appears in protein-free liposomes of phospholipid extracted from the various membranes. Although Hock and Taraschi [292] note that it is "not essential to maintain the physiological asymmetric distribution of membrane lipids, which is lost in reconstituted liposomes", cardiolipins maintain their asymmetric distribution by finding the concave inner surface of liposomes $[427,447]$.

In mixed-phospholipid liposomes prepared from liver mitochondria of ethanol-fed rats, only the cardiolipin fraction of the extracted phospholipids confers membrane tolerance to added ethanol; phosphatidylinositols from microsomes and phosphatidylserines frum synaptosomes act similarly in the corresponding mixed-phospholipid vesicles. We do not know the changes in the phosphatidylinositols or phosphatidylserines, but we do know that fatty acyls change in the cardiolipins. The mitochondrial phospholipids contain cardiolipins as 10\% of total phospholipids (and 46\% phosphatidylcholines, $42 \%$ phosphatidylethanolamines, $2 \%$ phosphatidylinositols + phosphatidylserines - about the proportions in preparations from control- fed animais); the ethanol-tolerance persists when the adidiolipin/phospholipid ratio is made as low as $3 \%$. It nay be relevant that ethanol interacts directly with the xadgroup region of anionic phospholipids and exerts alli ordering effect on membrane surface H-bonded networks [292] in which cardiolipins may play a role (sec section II-A).

IV-C.2.b. Magnesium deficiency: Mg-deficiency decreases liver microsomal Jo-desaturasc activily and (thereby?) raises the 18:2 acyl content in the phospholipids of microsomes [403-405] and mitochondria [509]. $\mathbf{M g}^{2+}$ ions associate with mitochondrial cardiolipins [526] and are cofactors in phosphatidylcholine biosynthesis. Therefore. reports that dictary $\mathbf{M g}$-deficiency uncouples oxidative phosphorylation [680] seem worth examining here. Heart mitochondria isolated fr'm young rats were uncoupled in 4-8 days of fecding a $\mathrm{Mg}$-deficient dict. with $\mathrm{P} / \mathrm{O}$ ratios 1.4-1.0 (a-ketoglutarate, temperature not specified). Oxidative nhosphorylation in liver or kidncy mitochondria was minimally affected. but both liver and hearl mitochondria from $\mathrm{Mg}$-deficient rats swelled faster at $37^{\circ} \mathrm{C}$ (but not at $200^{\circ}()$ than did mitochondria from controls [453]. However, $P, O$ ratios in heart mitochondria were normal uncler similar conditions although serum [Mg] was as low as 20) of normal: no respiratory rates were shown [32]. And Hegsted and coworkers [453] found normal amounts of $\mathbf{M g}$ in heart and liver mitochondria of $\mathrm{Mg}$-deficient rats.

Mg-deficiency damages mainl; the mitochondria of rat hearts; electron microscopy shows decreased niatrix density, swelling and myelin figures [269,638]. Damaged mitochondria in sitli also appear in EFA-deficiency and thyrotoxicosis (see Ref. 284) even though some in vitro assays of oxidative phosphorylation are within normal ranges. Mitochondrial defects in Mg-deficiency are still invoked: for example, cthanol-feeding leads :: hypomagnesemia, which is partly blamed for heart lesions of mitochondrial origin [56].

Even though mitochondria contain normal amounts of $\mathbf{M g}, \mathbf{M g}$-deficiency-induced alterations in mitochondrial phospholipids would persist. The enzymatic conversion of phosphorylcholine $\rightarrow$ phosphatidylcholine requires $\mathrm{Mg}^{2+}$. By the 4 th day of $\mathrm{Mg}$-deficiency, synthesis (mcasured by "P incorporation and lipid phosphorus) of heart mitochondria total phuspholipids and resolved phosphatidyl-ethanolamine, -serine, -choline, -inositol and sphingomyelin (?) decreases by some $50 \%$ [66]. Because the specific activity of total phospholipids was low although relative specific activities of thesc individual phospholipids was not much decreased, synthesis of some other undetermined lipid-soluble fraction was thought to be susceptible to $\mathrm{Mg}$-deficiency phosphatidic acid, an intermediate in phospholipid synthesis, was proposed but cardiolipins were also not analyzed in this study and seem more likely to accumu- 
late as an end-ploduct. This idsa is indircell supported by observations that liver mitcohondriat lipas from Mg-deficient rats. compared with controls. contain 12r; more $18: 2$ acyls [5(N)]. and mi rowomes conltain 25\% more $18: 2.50 \%$ more 211 : 31 - - hl. and $111 \%$; less 201:4 acyls. These tindings are sitributed 10 a

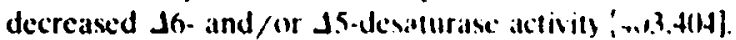
No direct information is availahle on cardiolipin composition or content in liver or heart mitcohondria in Mg-deficiency. but in kidney cells grown on low-[Mg] media. phosphatidylcholine increases while phosphatidylethanolamine, phosphatidylserine, phosphatidylinositol and sphingomyelin decrease [405].

Effects of Mg-deficiency should be difficult to detect in in vitro assays of oxidative phosphorylation. $\mathrm{Mg}^{2}{ }^{\prime}$ is required in the reaction mixture; the presence of 22.5 $\mu \mathrm{mol}$ of $\mathrm{MgSO}_{4}$ [32.(6\$1)] should reverse any deficit. Non-invasive in situ measurements by saluration transfer "P.NMR (see section IV.C.2.d) could be more definitive.

IV.C.2.c. Temperalure. The cukaryole basic stratcgies for coping with decreases in environmental $1 \mathrm{~cm}$ peratures involve keeping biomembranes functional (c.g., so that they do not congeal) and generating more heat ( $s$ ) that cell temperature does not get low enough to congeal membranes). These strategics converge in mitochondrial inner membranes, which both mediate energy transductions and generatc basal and extra heat. We know much less about metabolic adaptations to above thermoneutral environments than to below thermoneutrality. Cold-activated hormonal mechanisms mediated by local metabolites regulate mitochondrial function: in contrast, thermosensitive enzymes regulate membrane function and hes: production in prokary. otes (section III-A).

Living cells generate heat almost completely from highly exergonic oxidative reactions. 'Thermogenesis' denotes extra heat production above basal levels [519]. Rescarchers disagrec over thermogenic mechanisms. Sone early rotions, including those of the reviewer [278], and some current ideas: $[132,193,363,450,525]$ attribute extra heat production to uncoupling and the diversion of the free energy change of oxidations from driving the endergonic phosphorylation reaction, ADP $+P_{i} \rightarrow$ A'TP. Others point out that the caloric yield due to substrate oxidation is independent of the reaction sequence: "... any ATP synthesized by mitochondria within a cell is immediately recycled and does not appear in the final equation of reactants and products ..." [465] (see also Refs. 38,273$275,280,518.519)$. Therefore, respiration rate is the source and index of thermogenesis, and the chemical mechanism that sets respiratury rate can be considered the mechanism that regulates heat production [273$275,465,518,519]$. This argument scems the sounder, and is productive in depicting mechanisms whereby
I: |H| I: VI

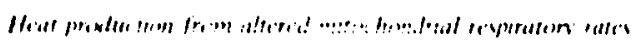

11 . enderences sec the lext.)

\begin{tabular}{|c|c|c|c|}
\hline 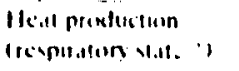 & Imses & $\begin{array}{l}\text { I Proumallux } \\
\text { nechianism }\end{array}$ & Rille \\
\hline \multicolumn{4}{|l|}{ II.19,1 h:.11 } \\
\hline St.ıle 4 & 1:11) & prollon lish & ++ \\
\hline Sitale? & an! & C, values & ++ \\
\hline \multicolumn{4}{|l|}{ Ixtrahen" } \\
\hline kxose-coupled (4) & an; & protomuphore & ++4 \\
\hline uncoupled $\left(?_{u}\right)$ & any & protone & $+++t$ \\
\hline cold shivering $(1)$ & muxile & $+\Lambda$ TPase & +++ \\
\hline \multirow[t]{3}{*}{ cond: metabulic (4) } & $\begin{array}{l}\text { liver. muscle } \\
\text { rat } B, A T\end{array}$ & $\begin{array}{l}\text { + PL -leak } \\
\text { + uncoupling }\end{array}$ & +++ \\
\hline & & protein & $+++t$ \\
\hline & ral $B \wedge T$ & - PL.lciak & + \\
\hline \multirow[t]{2}{*}{ I:F-deticiency (t) } & ralt liver & $+P l \cdot k$ alk & $++t$ \\
\hline & ril BAT & .PI.-kialk & + \\
\hline hyperihyroid (A) & liver & + PI. $/ F_{11} \cdot$ leak & $-+1+$ \\
\hline $\begin{array}{l}\text { malignant hymer. } \\
\text { thernatit }\end{array}$ & muscle & + X-leaks? & $+t+t$ \\
\hline \multicolumn{4}{|l|}{ I.c. heitl: } \\
\hline hỵmhyroid (t) & livet & -Pl. killk & + \\
\hline $\operatorname{diving}\left(5^{h}\right)$ & scal muxcle & buxconstriclionn & () \\
\hline
\end{tabular}

As defined hy (hance and Williams (xh):

" State s. defined as $\left[O_{Z}\right]=0$. here also includes $[S]=i$, in muscles.

membrane phospholipids, especially cardiolipins. may participate in extra heat generation by mitochondria.

Table VI summarizes some mechanisms whereby altered mitochondrial respiratory rates (proton flux) change heat production. Basal heat is produced from mitcchondrial Strte 4 respiration (nonphosphorylating) at the slow rate set by the proton leak, and from the more rapid (phosphorylating) State 3 oxidation rate regulated by the $C_{i}$ values of certain steps. Basal proton leakage and $C_{i}$ values differ among the mitochondria of various tissues; so do the phospholipids and cardiolipins; perhaps the two are connected. Different mitochondria also vary in thermogenic responses to temperature adaptations; perhaps this is also not unconnected with their phospholipid compositions.

A crucial test for a respiratory thermogenic mechanism is the existence of loose-coupling [381], the acceleration of State 4 respiration with continued synthesis of ATP. i.e., without uncoupling. By an 'uncoupling' mechanism, loose-coupling should not be thermogenic - but loosc-coupling is observed in mitochondria from thyrotoxic human and animal subjects $[641-643]$ that are in a veritable thermogenic state, i.e., with elevated BMR (oxygen). Loose-coupling produces extra heat when proton leakage is increased almost maximally but not enough to dissipate $\Delta p$ tellingly. Uncoupled oxidative phosphorylation (with $\Delta p$ insufficient for phosphorylation) produces extra heat at this faster, maximal rate set by a protonophoric mechanism; uncoupling-thermogenesis from State 3 respiration is usu- 
ally minor. since respiration is already near maximal because of the large influx of protons through the ATP-synthase. Thermogenesis starting from State 4 respiration ranges from 2-fold to 20 -fold. depending on the mechanism that increases proton icakage. This, and the fact that protonophores like dinitrophenol and the BAT uncoupling protein can triple the BMR in vive, strongly implies that most resting cells generate hassil heat from State 4 respiration rather that from St:.lc $3[278,280.597]$.

Ectotherms, with body temperatures that rapidly nedr changed ambient temperaturc. adapt to cold by increasing fatty acyl unsaturation in their membranc phospholipid: to maintain appropriate function [266. 268.399,527].

Unsaturation of mitochondrial lipids correlates inversely with growth temperature among yeasts [ $2(1,687])$. The mesophilic (growing between $\left(1^{\circ} \mathrm{C}\right.$ and $36^{\circ} \mathrm{C}$ ) yeast Candida lipolytica has mitochotidrial phosphatidylcholines that are more unsaturated when growth is at $10{ }^{\circ} \mathrm{C}$ than at $25^{\circ} \mathrm{C}$. and the phosphatidyiinositol, phosphatidylserine and phosphatidylethanolamine fraclions show lesser increases in unsaturation; little cardiolipin is present and its fatty acyl composition is not leported: unsaturation changes through a reciprocal relationship between percentages of $18: 1$ and $18: 2$ acyl groups [350,351,655]. A psychrophilic Candida growing at 0$17^{\circ} \mathrm{C}$ shows little change in fatty acyl composition. Saccharomyces cererisiac grown at $15^{\circ} \mathrm{C}$ or $30^{\circ} \mathrm{C}$ has phospholipids of similar fatty acyl composition, and cardiolipin / phospholipid ratios of about 4\%, but phosphatidylcholine/phospholipid is higher at the lower growth temperature [315]. Unsaturation index of total lipids of Saccharomyces grown at $40^{\circ} \mathrm{C}$ is 6.3 , at $26^{\circ} \mathrm{C}$, 45 [87]. Mitochondrial phospholipids from the thermophilic yeast Torulopsis bol ina include $25 \%$ cardiolipins: in psychrophile mitochondria cardiolipin/phospholipid is only $8 \%$ [20]. No fatty acyl compositions of cardiolipins are reported in these studies but we know that monounsaturated fatty acyls are $96 \%$ of the fatty acyl in mitochondrial cardiolipins of (presumably warm) Saccharomyces (see section IV-C.I.a).

Cold-adaptation $\left.(37-4)^{\circ} \mathrm{C} \rightarrow 15^{\circ} \mathrm{C}\right)$ induces some comparable alterations in the phospholipids of the fungus Neurospore crassa [1] and the protozoan $T e^{-}$irahymena pyriformis $[2(0,730]$. The relative amount of cardiolipins in Tetrahymena total phospholipids decreases from $14.5 \%$ to $9.9 \%$ (Table 111 ) and in Neurospora halves, which indicates that there are fewer mitochondria. In Neurospora and Tetrahymena mitochondria the cardiolipins per phosphulipids are about halved, indicating that membrane composition changes; Tetrahymena cardiolipins are replaced by phosphatidylcholines and phosphatidylethanolamines. The unsaturation index rises in Tetrahymena total phospholipids from 127 to 160, and in Neurospora mitochondrial phospholipids from 159 to 216 as $18: 3$ acyls substitute for 18: 2 acyls. Cold acclimation does not change $T e$ trahymena cardiolipin fatty acyls, which are quite unsaturated 'unsatusation index 2(K); see Table ill). In warm-cell mitochondria the phosphatidylethanolamines $[2(x)]$ and glycenl 2 -aminisethylphosphonolipids [3.37] are even more unsaturated: cold-exposure increases the unsaturation of these phospholipids rapidly and lincarly. and overall unsaturation index increases from 1.39 to 1 tor over the first $10 \mathrm{~h}$ [730]. Cold makcs the endothelial reticulum membrane lipids more rigid, which activales existing $\mathbf{4 9 - d e s a t u r a s e ~ e n z y m e ~ a n d ~}$ rapidly supplies more unsaturated fatty acyls; no new desaturase is induced, nor does the increase in dissolved $\left[\mathrm{O}_{2}\right]$ accelerate desaturations the way it does in hacteria [6(1)9.61(1)]. Cold adaptation does not alter the nuidity of extracted mitechondrial lipids, hut removal of cardiolipins from the phospholipids of cold (but not warm) toitochondria increases phospholipid fluidity; Yamauchi et al. [7.30] helieve that cardiolipins decrease fluidity in these mituchondria while the other phospholipids iacrease fluidity, and that cardiolipin reacts preferentrally with other phospholipids when they are more highly unsaturated. to counter overfluidization. An in. teraction between cardiolipins and the bulk phosphatidylcholines and phosphatidylethanolamines might also affect membranc barrier properties and accoun' for the apparent predominance of cardiolipin compositions in regulating State 4 proton ieakage across mitochondrial phospholipids (sce section II-A).

Flight muscle mitochondria from blowflies acclimated at $9^{\circ} \mathrm{C}$. as opposed to $24^{\circ} \mathrm{C}$. show increased fatty acyl unsaturation of total phospholipids; faster State 4 (proton leak) and State $3\left(C_{i}\right.$ values) respiration $\left(+50-9(1) \%\right.$, pyruvate/prolinc, measured at $\left.10-30^{\circ} \mathrm{C}\right)$ [133]: shifted inflections in Arrhenius profile of some electron carricrs demonstrate altered lipid-dependence.

Early studies on poikilothermic animals found no consistent effects of temperature changes on the unsaturation index of total phospholipids from mitochondrial membranes and mitochondrial respiration, although altcred Arrhenius profiles of respiratory rates implicated the lipids [666]. The apparent discrepancies are resolved by consideration of individual phospholipifs rather than iotal phospholipids, as suggested by various workers $[266,432,666]$. When the environmental temperaturc of goldfish shifts from $30 \rightarrow 1 C^{\circ} \mathrm{C}$, mitochondria from various tissues increase contents of phospholipid monounsaturated fatty acyls and polyunsaturated fatty acyls. Hazel [266] tests the ability of these temperature-altered phospholipids to reactivate delipidated succinate dehydrogenase from goldfish skeletal muscle mitochondria. Reactivation depends on whether the mitochondrial phospholipids, but not the enzyme, are from $25^{\circ} \mathrm{C}$ - or $5^{\circ} \mathrm{C}$-adapted goldfish: total 
phospholipids from $5^{\circ} \mathrm{C}$-mitochondria activate in $42 \mathrm{C}:$ of the in situ $V_{\max }$, total phospholipios from $25^{\circ} \mathrm{C}$-mitochondria, to $28 \%$. The efficacy of an isolated phospholipid subclass depends on its acidity: phosphatidyinositol $>$ cardiolipial $>$ phosphatidylscrine $>$ phosphatidylethanolamine $>$ phosphatidylcholine.

Wodtke [710-71.3] measures effects of cold-acclimation $\left(26-32^{\circ} \mathrm{C} \rightarrow 10^{\circ} \mathrm{C}\right)$ on the resolved phospholipids of liver and skeletal muscle mitochondria of carp. In liver mitochondria from warm-adapted carp. the unsaturation index of phosphatidylcholines and phosphatidylethanolamines is less than that of total phosphoiipids [712], so the cardiolipins (not measured) must be more highly unsaturated. Cold-adaptation does not alter cardiolipin/phospholipid, phosphatidylcholine/ phospholipid or phosphatidylethanolamine/phospholipid: comparisons of fatty acyl contents in total phospholipids with those in phosphatidylcholines and phosphatidylethanolamines indicates that the cardioiipins must lose 18:2 and 18:1 acyls, and gain saturated fatty acyls. In muscle mitochondria, cold-acclimation dous not change subclass phospholipid/total phospholipids ratios, including cardiolipin/phospholipid. or the unsaturation of the total phospholipids. However. measured cardiolipin unsaturation drops sharply (unsaturation index $214 \rightarrow$ 160: Table III) while phosphatidyicholine unsaturation index rises as markedly $(203 \rightarrow$ 249); in total phospholipids thesc opposing changes cancel out and thereby fatty acyl analyses of total phospholipids and resolved phospholipids lead to contradictory conclusions [714]. In frozen and thawed mitochondria from cold-acclimated carp. succinoxidasc activity is faster by $30 \%$, and cytochrome oxidase specific activity is about $50 \%$ greater while $\left[a a_{3}\right]$ and $K_{m}$ for cytochrome $c$ remain unchanged [713]; activation of cytochrome oxidase is attributed to phosphatidylcholine-induced augmentation of membrane fluidity rather than altered cardiolipin-oxidase interaction.

Tissues of goldfish $\mathrm{kept}$ at $10^{\circ} \mathrm{C}$ show the following differences from these kept at $30^{\circ} \mathrm{C}$. (i) Mitochondria prepared from gills contain total phospholipids that are more unsaturated and include more cardiolipins and phosphatidylethanolamines [71]; (ii) activities of their NADH-cytochrome-c reductase, succinate-cytochrome-c reductase, and cytochrome oxidase are higher when measured at $20^{\circ} \mathrm{C}$, while concentrations of $\mathrm{cy}$ tochromes $a a_{3},-b$ and $-c$ are unchanged [70]. (iii) Cytochrome oxidase activity (mitochondrial oxidative capacity) is increased at ali assay temperatures from $10-40^{\circ} \mathrm{C}$ in homogenates of gills, brain or muscle, but is higher in liver homogenates only when measured at $30-40^{\circ} \mathrm{C}$ [70]. (iv) Liver mitochondria have unchanged State 3 respiratory rates $\left(20^{\circ} \mathrm{C}\right)$ but decreased efficiency of phosphorylation [347]. Cold-acclimation $\left(22^{\circ} \mathrm{C}\right.$ $\rightarrow 12^{\circ} \mathrm{C}$ ) of eels slows liver cell mitochondrial respiration (succinate, measured at $25^{\circ} \mathrm{C}$ ) in State 4 although
ADP $/ O$ ratios also decrease $[710,711]$. However, the temperature of the assay is crucial for showing coldadaptation effects on mitochondrial respiration fas it is in demonstrating thyroid-state effects [49], sec section IV-C.4.d). Arrhenius profiles of State 4 and State 3 respiration change with cold-acclimation in such a way that rates are higher only when assayed at $<210^{\circ} \mathrm{C}$, in tench liver and muscle mitochondria 1521 and in frog skeletal muscle milochondria [52()].

Shivering is an carly thermogenic response in chilled homeotherms. Skeletal muscle contracts without doing work, maintains a high extramitochondrial steady-state [ADP] and $\left[P_{1}\right]$. and thereby accelerates respiration in the State $4 \rightarrow$ State 3 transition. Later responses to cold include thermogenic metabolic changes; in skele. tal muscles these may amplify or replace shivering thermogenesis. Skeletal muscle mitochondrial cardiulipins are relatively low in 18:2 and high in saturated fatty acyls centents in the Arctic mouse Microtus pennsylianicus kspt at $18^{\circ} \mathrm{C}$ (Table III), as compared with other Arctic mice. Cardiolipin saturated fatty acyls increase further while monounsaturated fatty acyls + polyunsaturated fatty acyl contents decline reciprocally hen Microtus adapts to a $5^{\circ} \mathrm{C}$ environment; these muscle cardiolipins resemble the liver cardiolipins of thyroid-treated rats in a typically thermogenic state. Skeletal muscles of cold-adapted rats have more mitochondria, repackaged to contain less cytochrome oxidase [34]; nevertheless State 3 specific respiratory activity (pyruvate/malate, $37^{\circ} \mathrm{C}$ ) rises $30 \%$ in both State 4 and State 3 [276]. Muscle mitochondria from coldadapted $\left(6^{\circ} \mathrm{C}\right)$ seals are loose-coupled with equal State 3 and 4 rates (succinate, $25^{\circ} \mathrm{C}$ ); in mitochondria from $201^{\circ} \mathrm{C}$-adapted seals State 4 rates are much slower than State 3 rates [223]. Cold-exposure of hamsters, but not of rats, increases liver mitochondrial respiration (succinate. $37^{\circ} \mathrm{C}$ ) in State 4 and State 3, as well as oxidative capacity (cytochrome oxidase) [83]. However, liver mitochondria from cold-adapted rats respire $30 \%$ faster in both State 4 and State 3 (succinate, $30^{\circ} \mathrm{C}$ ); $V_{\text {max }}$ of the ADP/ATP carrier is above warm-adapted levels, but only when measured at 25 or $37^{\circ} \mathrm{C}$ : i:nd $20: 4$ acyls replace a small fraction of $18: 2$ acyls in mitochondrial total lipids [4(6)].

Seals that dive for long periods arrest oxidations in skeletal muscles, probably by constricting arterial supply and thereby shutting down $\mathrm{O}_{2}$ and substrate supplies [579]. Reduced heat production in hypothyroidism, and thermogenesis in thyrotoxicosis, are discussed in section IV-C.2.d.

Major metabolic thermogenic adap.ations of mammals to low environmental temperatures occur in BAT. BAT raises body temperature far out of proportion to its limited localization and amount; a norepinephrine infusion increases BAT respiration 30-fold in normal rats and 80 -fold in cold-adapted animals where it con- 
tributes almost two-third: of the tripled BMR. Special features account for its thermigenic capacity: a proton-exchanger 'uncoupling' protcin is found only in the numerous large BAT mutochondria: the mitochondria are situated and equipped for latty acid cataholism. BAT mitcohondrial cardiolipins and phospholipids adapt to cold and diet hut, from one-temperature measurements. do not comsistently contribute to thermogenesis.

The activated uncoupling protein mediates thermogenesis by accelerating proton reflux (Table I); 'uncoupling' is not necessary (see above). BAT mitcchondria are rapidly and intensely thermogenic because a high content of uncoupling protein confers great protonophoric capacity. Uncoupling protein characteristics and rolics in adaptation of animals to cold environments, the neonatal statc, or overeating, have been reviewed $[73.274,463,465,615)$. The uncoupling proteins of rats, hamsters, guinca-pigs and rabbits crossreact immunologically. Although uncoupling protcin is genetically related, ADP / ATP carriers of beef heart and rat liver mitochondria do not crossreact with uncoupling protein antibodics [539].

The uncoupling protein, the ADP/ATP carrier and the $P_{i}$ carrier have repeated and related amino acid sequences, exist in dimer form in the membranc, and seem to share a common evolutionary origin, low hydrophobicity, three-dimensional structure, and transport mechanisms $[16,363,564]$. Uncoupling protein net positive charge is the lowest of the three. so it would presumably interact electrostatically least with cardiolipins; the $P$, and ADP/ATP carriers react specifically with cardiolipins (see section II-B). Unceupling protein purified from BAT mitochondria of coldadapted hamsters retains 0. (K $\mathrm{g}$ of phospholipids per $\mathrm{B}$ protein [39.3]. which would correspond to $1.2 \mathrm{~mol}$ of cardiolipins $/ \mathrm{mol}$. Solubilized pure uncoupling protein has been reconstituted by incorporation into liposomes made from phosphatidylcholines, pinosphatidylethanolamines and cardiolipin $(49.5: 49.5: 1)$ [6.34]. or from egg yolk phospholipids [364] which probably include some cardiolipin because they also reconstitute the cardi olipin-dependent ADP/ATP carrier. No systematic study of retained phospholipids, or of reconstitution efficiencies of various phospholipids, seems to be available.

Extramitochondrial purine nucleotides and protons are negative effectors, and fatty acids are positive effectors, for uncoupling protein exchange of protons [465]. GDP, GTP, ATP and ADP bind non-covalently and reversibly, without modifying (e.g., phosphorylating) the protein. One specific, high-affinity, saturable binding site on the protein dimer is on the outer face of the inner membrane where it is exposed to purine nucleotides in the cytosol and to whatever amounts of cardiolipins are on the outer face. Protons increase purine nucleotide hinding to the protein site. Free fatty acids in the order $16: 0>18: 1>18: 2>8: 0$ compete with MgAlp-binding to override the depressed proton conductance. There is no evidence that obserned upregulation of proton flux by hydronhobic sulfhydryl reagents [.5.38] is physiological.

Proton exchange across the active uncoupling pro. tein is nearly independent of $\mathrm{JpH}$, and is 100 slow to proceed via a proton channel mechanism [36.3]. The amount of activated uncoupling protein regulates proton flow $[463,465]$. Klingenherg $[36.3]$ proposes that the BAT uncoupling protein evolved late (i.e., in mammals) as an amputated $\mathrm{H}^{+}$/anion cotransporter (or $\mathrm{OH}$ /anion antitransporter) similar to a portion of the relaicid calriers of ADP/ATP and $P_{1}$, and shares features of their proton cotransport. We do not know if the similarity extends to a specific uncoupling protein interaction with cardiolipins that affects proton transport.

BAT is spccialized for thermogenic fatty acid catabolism. It! profuse sympathetic innervation and capillary vasculature promote activation of hormonesensitive lipase that supplics fatty acids from local and distant triacylglycerol stores. BAT mitochondria possess a very active set of $\beta$-oxidation enzymes; the staiturd enthalpy change for oxidation of 2 fatty acid carbons $\rightarrow$ acetyl-CoA is twice that of 2 glucose carhor.s $\rightarrow$ acetyl-CoA. The mitochondria contain little ATP-synthase and $s$ ) do not support a futile cycle that involves the plasma membrane NaK-ATPase [(6)5].

BAT mitochondria contain specialized phospholipids as compared with liver mitochondria. BA'l cardiolipins from rats, hamsters, and mice housed at $22^{\circ} \mathrm{C}$ are low in 18:2(n-6) acyls and repleted with saturated falty acyls (Table III). BAT mitochondria from rats and hamsters acclimated to different temperatures, and deprived of dietary EFA, are compared in Table VII.

Cardiolipins of rats kept at $22^{\circ} \mathrm{C}$ contain about $20 \%$ saturated fatty acyls. $40 \%$ monounsaturated fatty acyls and 40\%; polyunsaturated fatty acyls; phosphatidylcholines and nhosphatidylethanolamines have around $40 \%$ saturated fatty acyls and $30 \%$ each of polyunsaturated and monounsaturated fatty acyls; unsaturation indices in phosphatidylethanolamines $>$ phosphatidylcholines. Cold-adaptation from $28^{\circ} \mathrm{C}$ (thermoneutral) $\rightarrow 5^{\circ} \mathrm{C}$ increases polyunsaturated/monounsaturated fatty acyls ratios without changing the saturated fatty acyl groups of, any phospholipid in rat BAT mitochondria. Cardiolipins respond most. their polyunsaturated plus monounsaturated fatty acyls being greater than in phosphatidylcholines and phosphatidylethanolamines: polyunsaturated fatty acyls increase 80) (18:2 acyl content doubles) in cardiolipins. $70 \%$ in phosphatidylcholines and $50 \%$ in phosphatidylethanolamines. In addition, phosphatidylcholine/phos- 
phatidylethanoldmine ratios drop from $1.1 \% \rightarrow 0.76$; since phosphatidylcholines are less unsaturated, inner membrane overall unsaturation index increases further. Phosphatidylcholines and phosphatidylethanolamines are the major phospholipids, and thus seem responsible for the observed accompanying increase in membrane R.uidity $[72,73,591]$, a bulk property. State 4 respiratory rate $\left(25^{\circ} \mathrm{C}, \mathrm{GDP}\right.$-insensitive) decreases while a high $\Delta p$ persists: $C_{M}{ }^{\prime \prime}$ drops by $26 \%$ (Table VII). In the absence of Arrhenius plots to determine thermotropic properties, it appears that a diminished proton leak accompanies the cold-induced substitution of polyunsaturated fatty acyls for monounsaturated fatty acyis in mitohondrial phospholipids, especially cardiolipins: the rat BAT mitochondrial phospholipidleak does not seem to become thermogenic in the adaption tr: a cold environment. Cold-induced changes in BAT and other mitochondrial phospholipids may preserve bilayer milieu properties rather than barrier properties.

In all rat BAT mitochondrial phospholipids, EFAdeficiency markedly depletes $18: 2(n-6)$ and $20: 4(n$ - 6) acyls when rats are kept at $28^{\circ} \mathrm{C}$ or $5^{\circ} \mathrm{C}$ (Table VII). This loss of unsaturation is relieved partly by sharp increases in monounsaturated fatty acyl contents, partly by the appearance of $20: 3$ acyls, much less in cardiolipins than in phosphatidylcholines and phosphatidylethanolamines (as in liver mitochondria, see section IV-C.1.a). GDP-insensitive State 4 respiration (i.e., the phospholipid-leak) in BAT mitochondria de- creases with EFA-deficiency: $C_{M^{\prime \prime}}$ falls by $18 \%$ in $28^{\circ} \mathrm{C}$-rats and $5 \%$ in $5^{\circ} \mathrm{C}$-rats; inhibitor titrations reveal corresponding down-shifts in the relationship between proton flux and $I_{p}$ [220]: the rat BAT mitochondrial phospholipid-leak does not become thermogenic in the adaplive responses to EFA-deficiency. GDP-sensitive respiration increases. BAT mitochondria prepared from

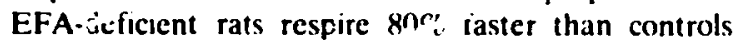
under 'State 4 ' conditions (pyruvate/malate, -GDP, $\left.25^{\circ} \mathrm{C}\right)(736]$ because uncoupling protein content accrues hy $46 \%$ and EFA-deficiency augments the protonophore-evoked respiratory chain capacity of rat BAT mitochondria by $60 \%$. The rat BAT mitcrhondrial uncoupling protein becomes thermogenic in response to cold-adaptation or EFA-deficiency.

BAT mitochondrial cardiolipins of hamsters kept at $22^{\circ} \mathrm{C}$, as compared with those of rats at $22^{\circ} \mathrm{C}$, contain $50 \%$ less 18:2 acyls, 30\% less polyunsaturated fatty acyls and twice the saturated fatty acyls [74] (Tables III and VIII). Hamster BAT mitochondrial phosphatidylcholines have about iwice the polyunsaturated fatty acyls and less monounsaturated fatty acyls. Cold-adaptation increases cardiolipin unsaturation by doubling the content of 18:2 acyls and polyunsaturated fatty acyls, and haiving saturated fatty acyls, but does not change fatty acyls in phosphatidylcholines or phosphatidylethanolamines. BAT mitochondria from coldadapted hamsters respire under State 4 conditions (glyccrol-3-phosphate, $23^{\circ} \mathrm{C}$ ) at $61 \mathrm{ng}$ atom $0 \mathrm{~min}^{-1}$ $\mathrm{mg}^{-1}, \Delta p$ is $<10 \mathrm{mV}$, and proton conductance via the

\section{TABLE VII}

Rat and hamster brown adipuse tissue and liter milochondria: fatty acyl unsaturations in cardiolipins, ptosphatidylcholines, and phos. pharidylethanolamines: State \& respiration and $C_{M}{ }^{\mu}:$ effects of temperature adaptation cind diefary essential fatty acid deficiency tcalculated from dcta of references shown in parentheses)

Alsbrevialious: BAT, brown adipose lissue; CL. cardiolipirs; EFA. essential fatty acids; PCs, phosphatidylcholines: PEs, phosphatidylethanolamines: PUFA. MUFA. SFA, polyunsaturated, monounsaturated. and sarterated fatty acyls: UI, unsaturation index.

\begin{tabular}{|c|c|c|c|c|c|c|c|c|c|c|c|}
\hline & \multicolumn{11}{|c|}{ Acclimation temperature $\left({ }^{\circ} \mathrm{C}\right)( \pm$ dictary EFA $)$} \\
\hline & \multicolumn{5}{|c|}{ rat BAT $[74,220,540,591]$} & \multicolumn{2}{|c|}{ hamster BAT $[74,460)]$} & \multicolumn{2}{|c|}{ rat liver [154.525] } & \multicolumn{2}{|c|}{ hamster liver [3,3] } \\
\hline & $28^{\circ}(+)$ & $22^{\circ}(+)$ & $59+1$ & $28^{\circ} 9-1$ & $50(-)$ & $\overline{22}(+)$ & $5^{\circ}(+)$ & $22^{2}+1$ & $22^{\circ}(-)$ & $22^{\circ}(+)$ & $22^{\circ}(-)$ \\
\hline CLs: PUFA & 26.6 & 43.8 & 48.5 & $5.7^{2}$ & $12.9^{\circ}$ & 30.6 & 53.1 & 53.4 & $21.3^{2}$ & 84.4 & 31.5 \\
\hline MUFA & 54.1 & 37.6 & 28.1 & 71.1 & 61.7 & 33.3 & 29.6 & 35.9 & 68.0 & 11.2 & 61.1 \\
\hline SFA & 19.3 & 18.9 & 23.0 & 20). 2 & 25.4 & 36.1 & 17.3 & 10.0 & 9.3 & 4.4 & 4.8 \\
\hline UI & 121 & 1.34 & 134 & 97 & 95 & 109 & 140 & 153 & 123 & 180 & 126 \\
\hline PCs: PUFA & 22.9 & 20.3 & 39.2 & $12.1^{\mathrm{h}}$ & $13.8^{\mathrm{h}}$ & 36.1 & 34.2 & 41.7 & $40.8^{n}$ & 38.8 & $33.3^{c}$ \\
\hline MUFA & $3 \times .2$ & 34.0 & 17.2 & 52.3 & 47.7 & 24.8 & 29.1 & 11.0 & $1 ! 0$ & 10.5 & 23.8 \\
\hline SFA & 38.9 & 45.7 & 41.6 & 35.6 & 38.5 & 39.0 & 36.7 & 46.0 & 48.2 & 50.7 & 43.6 \\
\hline UI & 110 & 91 & 126 & 90 & 87 & 106 & 127 & 16i) & 140 & 153 & 120 \\
\hline PEs: PUFA & 31.2 & 28.9 & 47.4 & $24.0^{\prime}$ & $34.4^{n}$ & 33.1 & 34.7 & 49.0 & $37.2^{n}$ & 23.9 & 20.2 \\
\hline MUFA & 35.8 & 28.5 & 14.3 & 47.0 & 31.2 & 30.9 & 30.5 & 6.3 & 15.1 & 22.8 & 45.8 \\
\hline SFA & 33.0 & $: 2.8$ & 38.3 & 29.0 & 34.4 & 36.3 & 33.2 & 43.7 & 45.7 & 53.0 & 33.0 \\
\hline UI & 153 & 132 & 185 & 128 & 148 & 116 & 127 & 133 & 137 & 90 & 110 \\
\hline State & $44^{d}$ & & $32^{d}$ & $30^{d}$ & $28^{\circ}$ & & $48^{d}$ & $20^{c}$ & $28^{\circ}$ & & \\
\hline$\Delta p(\mathrm{mV})$ & 228 & & 224 & 190 & 206 & & 220 & 186 & 187 & & \\
\hline$C_{M^{H}}+i$ & 1.16 & & 0.86 & 0.95 & 0.82 & & 1.31 & 0.66 & 0.90 & & \\
\hline
\end{tabular}

20:3 acyls are about " one third or less, ${ }^{h}$ half or ${ }^{c}$ four fifths, of these PUFA: ${ }^{d}$ substrate glycerol-3-phosphate, + GDP. + sfrum albumin, $25^{\circ} \mathrm{C}$ (ng atom $0 \mathrm{~min}^{-1} \mathrm{mg}^{-1}$ ): ' substrate succinate. $25^{\circ} \mathrm{C}$ : ' calculated usiug $\mathrm{H}^{+} / \mathrm{O}=6[220]\left(\mathrm{ng}\right.$ ion $\mathrm{H}^{+} \min ^{-1} \mathrm{mg}^{-1} \mathrm{mV}^{-1}$ ). 
uncoupling protein is $35 \mathrm{ng}$ ion $\mathrm{H}^{\prime}$ min $1 \mathrm{mg}{ }^{\prime} \mathrm{mV}$ ' $\left(\mathrm{H}^{*} / \mathrm{O}=6\right)[461]$. However, when the uncoupling pro. tein is blocked with GIDP and trec falty acids are sequestererl. Statc 4 respitation vat the phospholipidleak is $48 \mathrm{ng}$ alom $O \mathrm{~min}{ }^{1} \mathrm{mg}, . J p=220 \mathrm{mV}$, and $C_{M^{\prime \prime}}=1.31 \mathrm{ng}$ ion $\mathrm{H}^{\prime}$ min ${ }^{\prime} \mathrm{mg}{ }^{\prime} \mathrm{mV}{ }^{\prime}$. Although no BAT mitochondrial conductance is presented for hamsters kept at $22^{\circ} \mathrm{C}, \mathrm{C}_{\mathrm{M}^{\prime \prime}}=11.32$ (succinatc, $23^{\circ} \mathrm{C}$ ) in liver mitochondria from unadapted rats. Hamster BAT mitochondrial phospholipid-leak seems to become thermogenic as part of cold-adaptation.

Rat liver mitochondrial cardiolipins contain 20-75\% more polyunsaturated fatty acyls, about the same proportion of monounsaturated fatty acyls, and half the saturated fatty acyls found in BAT mituchondria (Tables III and VII). Phosphatidylcholines and phosphatidylethanolamines have about the same suturated fatty acyl content in liver and BAT mitochondria, hut in the liver organelfe phosphatidylcholine and phosphatidylethanolamine polyunsaturated/monounsaturated fatty acyl ratio is 4-7. whilst in BAT it is 0.7-1. Fatty acyl compositions of phospholipids do not seem to have been reported in liver mitochondria of cold-adapted rats. EFA-deficiency leaves rat mitochondrial phospholipid saturated fatty acyls unaffected, partly substitutes monounsaturated fatty acyls and only few 20:3 acyls for cardiolipin polyunsaturated fatty acyls, but exerts little effect on the unsaturation of phosphatidylcholines and phosphatidylethanolamines (see section IV-C.1.a). This constancy of the hulk phospholipids might account for the minor changes reported for bulk physical properties of liver mitochondria from EFA-deficient rats [284] and leaves the cardiolipin changes to account for effects on respiration. EFA-deficiency results in striking accelerations of rat liver mitochondrial State 4 phospholipid-leak (Table VII): $C_{M^{\prime \prime}} \cdot$ rises $36 \%$ ir. Cold-exposure of hamsters. but not of rats, increases liver mitochondrial respiration (succinate, $37^{\circ} \mathrm{C}$ ) in State 4 and State ?, as well as oxidative capacity (cytochrome oxidase activity) [83]. Hibernation in hamsters diminishes State 4 and State 3 respiration measured at $7^{\circ} \mathrm{C}$, but augments both rates when measured at $37^{\circ} \mathrm{C}$, and also cytochrome oxidase activity; these findings emphasize the need for thermotropic data on temperature adaptations. No information seems to be on hand on liver mitochondrial phospholipids in cold-exposed hamsters, or fatty acyl compositions of any mitochondrial phospholipid subclass in BAT of hamsters that go on to hibernate [10].

BAT responds to altered thyroid hormone levels. BAT cells have approximately the same specific T3-receptor binding capacity $(39,40,193]$ as white adipose tissue cells [67] and hepatocytes [482]. In rat BAT, unlike other cells, an intracellular $5^{\prime}$-deiodinase that converts T4 $\rightarrow$ T3 generates $55 \%$ of the nuclear-bound $\mathrm{T} 3$, which makes the BAT receptor relatively insensi- tive (1) plasma [T3] [39.40]. The deiodinase is stimulatcd hy extiamitochondrial signals that include $\alpha_{1}$ atgenists and other hormones. Receptor cecupancy determines expressior of the uncoupling protein and thereby BA $\mathrm{C}$ adaptation to cold environments.

Low temperatures evoke tinyroid hormone secretion necessaty for cold-adaptation: hypothyroid rats do not survive in cold environments [587]. Many steps in the sequence of events that controls the uncoupling protein are thyroid-responsive in BAT and other tissues (sec Ref. 284): the number of $\beta$-adrenergic receptors and their accesibility to norepinephrine; coupling of occupied receptors, via G-proteins, to adenylate cyclase (hypothyrcidism increases the sensitivity of the $G_{1}$-protein in BAT that couples inhibitory agonists (c.g.. adenosine) to adenylate cyclase 1723]); adenylate kinase activity: (ytosol [CAMP]; protein kinase-mediated phosphorylation of lipasc; conversions of triacylglycerol-fatty-acyl $\rightarrow$ frec fatty acid $\rightarrow$ fatty acyl-CuA that regulates [fren fatty acids]: the ratc-limiting steps in de novo acetyl-CoA $\rightarrow$ fatty acid: and the desaturalions of fatty acyl-CoA that lead to unsaturated free fatty acids that are less effective in activating the uncoupling protein.

Hyperthyroidism in rats kept at room temperatures affects the following processes in BAT. (i) Occupancy of BAT T3-receptors rapidly stimulates lipogenesis, which is already intense [339]; lipogenic enzyme activities double $[215,508]$; mitochondrial $\alpha$-glycerophosphate dehydrogenase activity. normally some 6()-fold higher than in liver, further increases by $50 \%$ [362]. (ii) Fatty acid $\beta$-oxidation and succinate oxidation in State $4\left(25^{\circ} \mathrm{C}\right)$ nse 4-fo!! [215]: the mitochondrial ou: rr carnitine long-chain-acyl-CoA transferase that reguiates $\beta$-oxidation in liver and other tissues is thyroid-sensitive and cardiolipin-dependent (see Ref. 284). (iii) BAT proliferates (white adipose tissue atrophies [7(12]) but cytochrome oxidase and cardiolipin/mg BAT remain unchanged [540], which denotes a constant content of mituchondria. (iv) Although T.3-treatinent (0.3 $\mu \mathrm{g} \mathrm{g}^{-1}$ day ' $\times 5$ d) increases the percentiges of $18: 2$ and 20): 4 acyls in total phospholipids of UAT mitochondria hut not BAT microsomes [215], more vigorous and prolonged T4-treatment of rats $\left(0.5 \mu \mathrm{gg}^{-1} \mathrm{day}^{-1} \times 40\right.$ days) doe's not change BAT fatty acyl/cardiolipin composition or cardiolipin/phospholipid ratios [446] (Table VII). The low-18:2, high saturated fatty acyl pattern of BAT cardiolipins characterizes tissues that do not respond thermogenically to thyroid hormone levels [284]. (v) Thyrotoxicosis depletes BAT uncoupling protein in warm rats $[637,662]$, and blocks cold-exposure from injucing uncoupling protein: it is a condition with negative survival value in ats at lo.v environmental temperatures [83].

Brown adipocytes from hypothyroid rats contain 3fold more mRNA $A_{\mathrm{S} / 4}$ (an intermediate in lipogenesis 
initiation) than euthyroids. Increased activities of BAT fatty acid synthase. ATP-citrate lyase and glycolytic flux lead to an 11-fold rise in falty acyl synthesis $[26,193]$. White adipose tissue from the same animals has only $20 \%$ of normal levels of mRNA $\mathrm{s}_{\mathrm{S14}}$ and shows no increase in fatty acyl synthesis; liver lipogenesis decreases. It is surprising that both hypothyroidism and hyperthyroidisn, augment lipogenesis in BAT. Hypothyroidism doubles ur triples activity of the BAT peroxisomal non-phosphorylating $\boldsymbol{\beta}$-oxidation apparatus plus catalase [210]. This system normally contributes $1 \%$ to $30 \%$ of the thermogenesis of overall fatty acid oxidation, and also accelerates during cold-exposure [72].

Malignant hyperthermia is a lethal syndrome of excessive mitochondrial heat production acconpanied by skeletal muscle rigidity. seen rarely in sensitive humans and more often in pigs of the German I andrace strain, when 'stressed' or exposed to halocarbon anesthetics [92-94.231),280]. Respiration of skeletal muscle mitochondria from malignant-hyperthermiasensitive pigs is abnormally stimulated by $\mathrm{Ca}^{2}+\mathrm{In}$ volvement of the unusually saturated muscle cardiolipins (Table III) and phospholipids (see section IV-B.2) of this and other strains of pigs does not seem to have been considered, although the increased mitochondrial $\mathrm{Ca}^{2+} / \mathrm{H}^{+}$exchange-leak shows abnormal lipid-dependency.

Hypothyroidism is accompanied by changes in B AT of $25^{\circ} \mathrm{C}$-adapted rats, and mice [316], that resemble the effects of cold exposure: increased BAT weight, DNA. and mitochondrial cytochrome oxidase (see Ref. 4(1,5); the last two are also unusual in hypothyroidism. Hiswever, hypothyroidism abolishes the increased respiration of BAT normally effected by norepinephrine or by exposing mice to the cold [293]. Cold exposure is lethal, and produces no lipolysis in BAT or increase in BAT mitochondriai uncoupling protein. Low, maintenance dosage of T4 ailows cold-induced synthesis of uncoupling protein. BAT is also unreactive in hypophysectomized rats and $o b / s h$ mice. Combined maintenance of hypophysectomized rats with $\mathrm{T} 4$ and corticosterone permits cold-adaptation and production of mitochondrial uncoupling protein. Although T4-treatment increases uncoupling protein in ob/ob mice, it does not permit cold-induced thermogenesis in BAT or save the cold $m \cdot e$ [274]. Mitochondrial phospholipid fatty acyl chang. 1 cold-adaptation are not consistently thermogen $n$ BAT but may be in liver (see above). Therefore, observed defects in hepatic fatty acyl-CoA jesalurative mechanisms may contributc to the hypothyroid's cold-sensitivity [284]; normal cats also fail to acclimate [46], and they have no J6-desaturase and

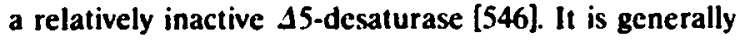
concluded that thyroid hormone is necessary but not sufficient for cold adaptation.

IV-C.2.d. Hormonal state. Thyroid: thyroid hormone levels and mitochondrial respiration (especialty Stat: 4) correlate dinectly in rat liver, heart and kidney [277.278]. and human skeletal muscle [6.32], tissues in which mit). chondria have high cardislipin/phospholipid ratios, cardiolipins have high $18: 2$ contents, and nuclei possess many T.3-receptors [284.285]. A uniform hypothyroid state is readily achicved by arresting hormone synthesis, but the effects of hormone administration or excess depend on several variables.

In the studics of Withers and Hu!bert [708] on liver mitochondri : of hypothyrsid rats, fatty acyl compositions of total lipids were close to normal and did not change when the rais were fed a control diet plus saturated or unsaturated fatty acids; they concluded that the hormone acts at least in part directiy on mituchondrial membrane lipids.

In our hypothyroid rats, liver mitochondral phospholipids show an abnormally high-12:2, low-20:4 fatty acyl pattern $[97-99,291]$ that reflects in part a $72 \%$ increase in cardiolipins with normally high $18: 2$ contents and no 20:4 acyls, in part the abnormal compositions of the phosphatidylcholines and phosphatidylethanolamines [291]. Raederstorff et al. [524] have recently confirmed this hormone-responsive pattern of abnormality in ( $n-6)$-unsaturated fatty acyls in the phosphatidylcholines and phosphatidylethanolamines of mitochondria from livers of hypothyroid rats, and also find significantly decreased contents of 22: $6(n-3)$ acyls. Lack of thyroid hormone usually raises levels of metabolic intermediates by slowing their removal more than their synthesis. It is therefore of interest that cardiolipin accumulates in plasma membranes of $\boldsymbol{H}$. infiuenzae when activity of a cardiolipinspecific phospholipase D is inhibited (see section III-A). Phospholipase D enzymes exist in rat liver endoplasmic reticulum [665].

The data are not sufficient to decide if cardiolipins, or phosphatidylcholines and phosphatidylethanolamines, or both, contribute to the slowed phospholipid-leak that characterızes hypothyroidism. Studies on induced changes in mitochondrial phospholipids (section IV-C) indicate a specific role for cardiolipins. The sidedness of the extra cardiolipins in the inner membranes of mitochondria from hypothyroids (or hyperthyroids) has not been examined, but the unsaturation of cardiolipins influences sidedness. The normal fatty acyl profiles of the excess cardiolipins should favor inner-sidedness. Supporting this inference are the folluwing observations on the outer-face mitochondrial carnitine palmitoyltransferase: its activity and susceptibility to physiological inhibitors have characteristics of lipid-dependency $[366,741]$; cardiolipins specifically activate the purified enzyme and may orient the mitochondrial enzyme [470]: activity is abnormally low in hypothyroids (see Ref. 284).

In hypothyroidism, 18:2 acyl contents rise and 20:4 
contents fall reciprocally not only in liver mitixhondria but also in the phospholipids of microsomes and nuclear envelopes. [181.2\%1.595]. The pereaciveness of these alterations has been attributed to the observed defects in the desaturation steps of the conversion of 18:2-CoA $\rightarrow 20: 4-\mathrm{CoA}[284]$. The diminished incorporation of administered $18: 2$ fatty acid into liver phospholipids [291] dow: no! seem to iimit $18: 2$ acyl accumulation in liver organelle phospholipids. Nor does the failure of the fasted-refed hypothyroid rat to induce hepatic 19-desaturase [288] depletc $16: 1$ and 18:1 fatty acyis.

To exatnine effects of thyroid hormone. several dose-time schedules have evolved. Originally, treatment of normal cuthyroid rats with a very high dose of DL-T4, equivalent to $70 \mu \mathrm{g}$ of the effective component L-T 4 per g bedy weight, given over 4 days, increased liver mitochondrial State 4 respiration $\left.(31)^{\circ} \mathrm{C}\right)$ and abolished phosphorylation of ADP [2910.414]. Latter, one much smaller dose of $T 3$ given to a hypothyroud rat was observed t.s stimulate mitochondrial State 3 respiration and synthesis of liver phospholipids, RNA, and proteins after 2 days [642,643]. Even more rapid effects in respiration are reported: onc injection increases State 4 respiration in $2-15 \mathrm{~min}$ in hypothyroid rats, and in 6-24 $h$ (but not at 48-72 h) in cuthyroid rats [279]: perfusion of hypothyroid rats' livers with T3 progressively increases State 3 respiration to a maximum at 60 min [586]; T3 affects cultured hepatocytes in $2 \mathrm{~h}$ [56.3]. 'Hyperthyroidism' is now often produced by 10-21 daily injections into euthyroid rats. Only a few of the later treatment schedules can be inte; rated with studies on phospholipid mutaholisril a!: 1 resolved mitochondrial phospholipids.

Thyroid-::eatment very rapidly !äus. is increascúd activitics of lipogenic enzymes [482] and fatty acyl-COA 19-, J6- and 35-desaturases, and to altered mitochondrial cemposition and function [284,285]. After one injection into a hypothyroid rat. T3-occupancy of T3depleted nuclear receptors in lipogenic tissues begins to activate in minutes. and then induce synthesis of. the depleted fatty acid synthetase. acetyl-CoA carboxylase, and supporting NADP ${ }^{-}$-reductases [482]. Saturated fatty acyls. perhaps those newly synthesized. substitute for many of the $18: 2$ and $20: 4$ acyl ron'ps in total phospholipids of liver mitochondria by $1-2.5 \mathrm{~h}$ [284] but therc is no information on cardiolipins this early. In contrast, in normal rats fasted and refed carbohudrates. monounsaturated fatty acyls replace liver mitochondrial total phospholipids $18: 2$ and $20: 4$ acyls even more severely by 2 days [9]. T3-treatment of euthyroids on cach of 5 days substitutes saturated fatty acyls only in cardiolipins; mitochondrial phosphatidylcholines and phosphatidylethanolamines become slightly more unsaturated because 20:4 acyls replace some of the 18:2 acyls [378] (Table III). This hyper- thyroid patiern of cardiolipins with low $18: 2$ and high saturated fatty acyl contents is unique among agents that change liver cardiolipin fatty acyl distributions. Microsomal phospholipid fatty acyls re nain unchanged after 5-days treatment [56.3] hut show the mitochondrial pattern after a 21-days schedule [180]. To the present, we do not know the mitcchondrial phospirolipid compositions that accompany uncoupling proton f?:ux after high doses of hormone.

Conversion of $18: 2 \rightarrow 20: 4$ acyls, which would account for the low 18:2 and high 20:4 pattern by accelerated microsomal $\mathbf{5 0}$ - and 55 -desaturations, occurs transiently in 1-4 h in T3-treated hypethyroid rats, and after 3 days of treatment in euthyroid rats. The 59-desaturase conversion of 18:0 $\rightarrow 18: 1$ acyls intensifies but seems not to affect cardiolipin or other phospholipod compositions, probably because $18: i$ acid is so available from the diet $[99,181]$.

Hyperthyroidism in rats also stimulates almo.t all the successive steps in hepati: metabolism of tatty acids and phosphoglycerols, and mitochondrial cardiolipin synthesis [284]. Cardiolipin, phosphoiipio ratios arc ainormally high $\left(+50 C_{i}^{\prime}\right)$ in liver and ticart mitnchondria after normal rats are treatec with T3 for 5 days [56.3]; a 3-day schedule did not alter heart cardiolipin / phospholipid proportions [28.3]. Increased cardiolipin/phospholipid ratios could result from a greater increase in cardiolipin synthesis than in phosphatidylcholine and phosphatidylethanolamine synthcsis. Another possible mechanism. activation of a mitochondrial phospholipase $A_{2}$ by elevated levels of thyroid hormone [44.3] and cardiolipins [387], would remove phosphatidylcholines and phosphatidylethanolamines with high polyunsaturated fatty acyls contents hut leave cardiolipins unljed [576].

Th: decreased unsaturation of the cardolipins from hyperthyroids should remove the conical shape that makes cardiolipins seck concave hydrophobic surfaces. Amounts of cardiolipin in the outer face of mitochondrial inner membranes do indesd secm is be increased in hyperthyroids. as judged from activity of the liver mitcohondrial 'outer' carnitine palmitoyltransferase (sec Ref. 284):

Recent studies define thyroid effects on mitochondrial State 4 respiration in terms of proton conductance, driving force $د p[54,246-249]$ and inner membrane area [249]. Hypothyroidism. depressis proton leakage estimated from either FCCP-titrations [228.673] or State 4 rates (succitiate, 25 or $37^{\circ} \mathrm{C}$ ) multiplied by the $\mathrm{H}^{+} / \mathrm{O}$ quotient (Table VIHI).

The slowed leak results from decreased proton-conductance of the membrane, since hypothyroidism does not alter $\Delta p$ in correlation with State 4 rates. $C_{M}$ ! $\cdot$ for ruthyroid rat liver mitochondria (succinate, $25^{\circ} \mathrm{C}$ ) is typically $0.3-1 \mathrm{ng}$ ion $\mathrm{H}^{\cdot} \mathrm{min}^{-1} \mathrm{mg}^{-1} \mathrm{mV}^{-1}$ [464]. $C_{M^{2}} \cdot$ is $0.63^{2}-16$ ? in Tahle VIII, perhaps because 
$C_{M^{\prime \prime}}$ tends to increase with temperature. $C_{\mathrm{Aq}^{\prime \prime}}$ for mitochondria from hypothyroid rats $(0.74-(0.9)$ is consistently lower than in euthyroids and deres not vary much with temperature in the few measurements shown. Brand and coworkers $[248,450]$ conclude that the slowed preton leak in hypothyroidism accounts for the entire dirsinished proton permeability. making it redundant to postulate a decreased $\mathrm{H}^{+} / \mathrm{O}$-slip.

The effects of hormune administration depend on the initial thyroid state, the dose-time schedule, and perhars the conditions of assay. In liver mitochondra from hypothyroid rats given one dose of T3 1 day before killing, proton leakage increases $3(0-4) \%$ as estimated from FCCP-titrations or State 4 respiration $\left(25^{\circ} \mathrm{C}\right)[298-300]$ (Table VIII). The excess leak is not via the $F_{0}$ : oligomycin actually increases State $4 t y$ $26 \%$. Treatment raises the proton driving force $J p$ mainly by increasing $\mathrm{JpH}$ to $81 \mathrm{mV}$ (an unusually high level considering the presence of $P_{1}$ ); the resultant $C_{M^{\prime \prime}} \cdot$ rises to normal levels. Introduction of prosonophores would increase proton leakage: one propused source is autoxidation of unsaturated fatty acyl groups in membrane phospholipids (sec section 11-A). Produets of mechanisms that protect atgainst superusides (e.g. oxidized glutathione and malondialdehyde) do not increase $24 \mathrm{~h}$ after hypophysectomized (and so also) hypothyroid) rats are injected with 13 . although the proton leak (State 4 respiration) accelerates 2.2-fold [401]. Thus, lipid peroxidation does not seem to cause this augmented prisien leak.

Treating normal rats with 1.5 fio: 10 days "hypeithyroids $)$ increases liver mitochondrial State 4 respiration $\left(37^{\circ} \mathrm{C}\right) 64 \%$ in the studies of Hafner et al. [244]. The increased leak, as measured by State 4 respiration, is not inhibited by oligomycin and so does not proceed across $F_{10}$ : the leak was not measured by FCCP-titration. $C_{M^{H}}$. for all mitochondria froni hyperthyroids is in the range $0.84-2.38 \mathrm{ng}$ ion $\mathrm{H}^{*} \min ^{-1} \mathrm{mg}{ }^{1} \mathrm{mV}$ '. consistently greater than control values (Table VIII). The major proton drive is $\rfloor \psi$ (which treatment diminishes by $14 \mathrm{mV}$ ). since the presence of nigericin and $P$, collapses most of the $1 \mathrm{pH}$ (only $15.9 \mathrm{mV}$ in cuthyroids

\section{TABLE VII!}

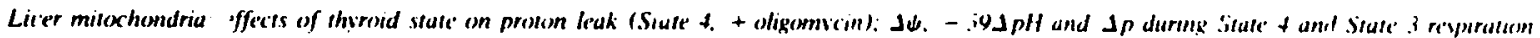
(succinale or succinute / malate), calculation of effectis e proton conductance ith?2).

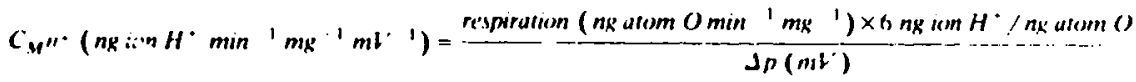

Abbreviations: ET, thyroid-trejted cuthy roids: IT, thyroid-treated hypothyroids: oligo. oligomycin

\begin{tabular}{|c|c|c|c|c|c|c|c|c|}
\hline \multirow[t]{2}{*}{ Thyroid state } & \multirow[t]{2}{*}{$\begin{array}{l}\text { Protun leak } \\
\text { (nmu! FCCP, } \\
\text { mg) }\end{array}$} & \multicolumn{2}{|c|}{$\begin{array}{l}\text { Respiration } \\
\text { (ag atom O } \\
\text { min ' mg ') }\end{array}$} & \multirow[t]{2}{*}{$\begin{array}{l}j \psi \\
(m V)\end{array}$} & \multirow[t]{2}{*}{$\begin{array}{l}-54 \mathrm{dpl} \\
(\mathrm{mV})\end{array}$} & \multirow[t]{2}{*}{$\begin{array}{l}\text { sp } \\
(\mathrm{mV})\end{array}$} & \multirow[t]{2}{*}{$C_{411}$} & \multirow[t]{2}{*}{ 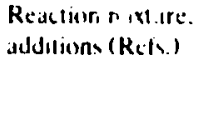 } \\
\hline & & - oligo & +olig. & & & & & \\
\hline Euthy:a:d & & 211 & & $|\times|$ & 14 & 145 & 1.63 & $\begin{array}{l}\text { State 4. 31) }(1, \\
\text { valinomycon }\{548\}\end{array}$ \\
\hline ET (TAsc $X \mu R g \quad+1 d !$ & & 30 & & 144 & 17 & 211 & 11.84 & \\
\hline Euthyroid & & lik & & 103 & 10 & 113 & 5.61 & Stive $3+A D P$ \\
\hline ET (T tsc K $\left.\mu g g^{\prime}+1 d\right)$ & & $\cdot 11$ & & 1113 & $x$ & 111 & 7.62 & \\
\hline Euthyroid & $i j .8$ & $\therefore$ & & & & & & 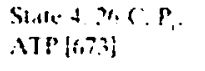 \\
\hline Hypothyroid & 4.5 & 33 & $2:$ & ji4 & & & $1: 1+1 \div$ & \\
\hline HT (T3ipnd & $5 x$ & 46 & 35 & Ith, & & & $(-! .84)$ & \\
\hline ET (Ttip $\left.x \mu \& \varepsilon^{1}+1 d\right)$ & & 62 & & & & & & \\
\hline Euthyroid & $n . x$ & 38 & 42 & 1.31 & 71.7 & 2015 & 1.018 & $\begin{array}{l}\text { State 4. 25. C: } \beta_{1} \text {. } \\
\operatorname{ArP}[24 \times-3(x)]\end{array}$ \\
\hline Hypothyroid & 5.1 & 29 & $2 x$ & 125 & 73.7 & $1 W$ & $0 . K Y$ & \\
\hline 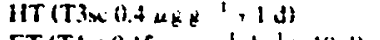 & 2.3 & 35 & +4 & 127 & 81.0 & 2018 & 10.98 & \\
\hline ET (TAsc $0.15 \mu \mathrm{BE}$ 'd $\left.{ }^{\prime} \times 10 \mathrm{~d}\right)$ & h.h & 53 & 46 & 133 & $(x) .4$ & 214 & 1.27 & \\
\hline Euthyroid & & & 49 & $2(x)$ & 15.4 & $21 \mathrm{th}$ & 1.22 & $\begin{array}{l}\text { State } 4.37 \mathrm{C}, \mathrm{P}_{1} \text {. } \\
\text { nigericin }|249|\end{array}$ \\
\hline Hypothyroid & & & 39 & 214 & 11.8 & 232 & 11.74 & \\
\hline ET (T3 $0.15 \mu g g^{\prime} d^{\prime} \times[(1)$ & & & $\$$ & $18 x$ & 18.7 & 202 & $2.3 k$ & \\
\hline Euthyroid & & 187 & $(52)^{\star}$ & 166 & 25.5 & 192 & 5.84 & $\begin{array}{l}\text { State } 3.37^{\circ} \mathrm{C} \\
\text { ADP }[120]\end{array}$ \\
\hline Hypothyroid & & 95 & $(29)^{*}$ & 174 & 11.6 & 191 & 2.46 & \\
\hline HT (T3iv $\left.0.15 \mu \mathrm{g} \mathrm{B}^{\prime \prime}+15 \mathrm{~m}\right)$ & & 108 & $(2 x)^{*}$ & 180 & 18.7 & 194 & 3.26 & \\
\hline
\end{tabular}

* State 4 respiration calculated from values shown for State 3 and respiraion control ratios. 
and $2.8 \mathrm{mV}$ higher after treatment). Because $1 p$ is in the order hypothyroid > cuthyroid > hyperthyroid. whilc respiration is in the order hyperthyroid > euthyroid $>$ hypothyroid, these workers ascribe the differences in State 4 rates at least in part to changes in the area of the inner membrane (per $\mathrm{mg}$ mitochondrial protein). By stercological electron micrographic analyses, the area of cristac was found to be $25 \%$ below normal in mituchondria of hypothyroid rats, as was the ratio of cardiolipin/mitochondrial protcin [326]. Wc found a mitochondrial cardiolipin/phospholipid ratio 72\% higher than normal [291]; the increased normal cardiolipins might increase proton retention in addition to the effects of decreased conductance area. Jakovcic et al. [326] made rats hyperthyroid by administering $\mathrm{T} 4$ over 21 days, cnough time to induce a new population of mitochondria with augmented cristael area, and perhaps comparable with the 10-day treatments shown in Table VIII. It seems doubtful though that 1-day to 3-day treatments produce new mitochondria, which leaves the proton permeabilities rather than membrane area to account for such carly stimulations of State 4 respiration.

Horrum et al. [298-300] find that 10 daily T4 injections accelerate State 4 respiration $\left(25^{\circ} \mathrm{C}\right)$ in the absence of oligomycin, although they do not increase State 4 or the degree of proton leakage measured by FCCP-titrations in the presence of oligomycin. Oligomycin inhibits only the increased portion of State 4 respiration; ergo, an $F_{10}$-leak accounts for the rise in proton flux above the persisting basal proton-leak. As calculated from $\Delta p$, the hyperthyroid state raises $C_{M^{\prime \prime}}$. from 1.08 (control) to $1.27 \mathrm{ng}$ ion $\mathrm{H}^{+} \mathrm{min}^{-1} \mathrm{mg}^{-1}$ $\mathrm{mV}^{-1}$. Despite the presence of $10 \mathrm{mM} \mathrm{P}_{\mathrm{i}}, J \mathrm{pH}$ is relatively high in euthyroids $(74.7 \mathrm{mV})$ and $6 \mathrm{mV}$ greater in the hyperthyroid mitcchondria, whilst $j \psi$ hardly changes. Thus, a T3-induced $F_{10}$-leak appears to be selectively driven by $\Delta\left[\mathrm{H}^{+}\right]$. If the lowered unsaturation of the cardiolipins observed at 5 days of T3. treatment [563] continues to 10 days, it is not associated with an increased phospholipid-leak, which contrasts with findings in EFA-deficiency and other conditions. If FCCP-titrations do i.ldex the proton ledk, which is disputed [62], these observations also seem to conflict with those of Nicholls [459] that proton passage through the $F_{v}$-channel in the presence of $A D P+P_{i}$ decreases $\Delta \mathrm{pH}$ enough to eliminate proton leaks; further, hyperthyroidisin raises $\mathbf{A p H}$ (Table VIII). The alternative mechanisms, whereby T4-treatment of normal rats initiates an $F_{0}$-leak when $\Delta \mathrm{pH}$ is allowed to contribute to proton drive, or T3-treatment increases other leak routes when $\Delta \psi$ is made to predominate [249], should be resolved by treatment of rats with either $\mathrm{T} 4$ or $\mathrm{T} 3$, and measurements at 25 and $37^{\circ} \mathrm{C}$, \pm oligomycin.

A significant $F_{0}$-leak in hyperthyroid mitochondria under State 4 conditions might be considered an accelcration of a nornal proton route through $F_{0}$ [415]. These studies report an impressive degree of $F_{0}$-leak in the very slow respiration of normal rat liver mitochondria $\left(+\right.$ substrate, $O_{2}$ and $P_{1}, 25^{\circ} \mathrm{C}$ ) (sec Table VIII). Oligomycin inhibits State 4 respiration by approx. $50 \%$ and raises $\Delta \psi$ to $187 \mathrm{mV}$ from a level of 176 $\mathrm{mV}$. The corresponding effective proton conductances can be calculated to be $<0.20$ (since $\Delta \mathrm{pH}$ was not measured) $\mathrm{ng}$ ion $\mathrm{H}^{+} \mathrm{min}^{-1} \mathrm{mg}^{-1} \mathrm{mV}^{-1}$ plus oligomycin and $<0.43$ minus oligomycin. Oligomycin inhibits State 3 by $90 \%$, to leave a nonphosphorylating respiratory rate that is $30 \%$ below the uninhibited Statc 4 rate. Masini et al. [415] concluded that a large portion of State 4 respiration is normally linked to rephosphorylation of intrinsic ATP used in matrix reactions like pumring $\mathrm{Mg}^{2+}$ and changing membrane conformation. However, the mitochondrial concentrations in these studies were 3-3.5 mg of protein per $\mathrm{ml}$, and the State 4 rates are as low as $25 \%$ of those measured at $25-26^{\circ} \mathrm{C}$ by other workers cited in Table VIII, who used 0.13-2 mg mitochondrial protein per $\mathrm{ml}$ (cf. Estabrook [178]: $1.7 \mathrm{mg} / \mathrm{ml}$ ) and saw no oligomycin inhibition of State 4 respiration in controls. Similarly, Shears and Bronk [598]. who uscd $4 \mathrm{mz} / \mathrm{ml}$, reported a State 4 rate at $30^{\circ} \mathrm{C}$ that is half that found by the others at $25^{\circ} \mathrm{C}$. It thus appears that $\left[\mathrm{O}_{2}\right]$ may have limited respiration in Masini et al.'s measurements, and thereby activated ATPase.

Altered thyroid states induce much larger changes in State 4 respiration than in $\Delta p$, apparentiv by altering the supralinearity of proton conductance at the high $J_{p}$ values characteristic of State $A$ icspiratir $n$ [53,54.246]. Supralinearity has been ascr:bed to several mechanisms: dielectric breakdown of membrane resistarse to proton current [459]; intramembranal proton circuits not mediated by $I_{F}[488,556]$; lateral proton conductance mediated by cardiolipins and $\mathrm{PGP}_{11}$ on surfaces of bacterial membranes (see section II-A); and properties of membrane lipids whereby (saturateo') phospholipids pass protons transmembranally along a transient hycrogen-bonded chain of water molecules (sec section 11-A). Mitochondrial $C_{\mathrm{M}^{\prime \prime}}$ is ohmic in cuthyroids to about $180 \mathrm{mV}$, in hypothyroifs to 200 $\mathrm{mV}$. and in hyperthyroids to $160 \mathrm{mV}$. Thus, the susceptibility of inner membrane to brcakdown at high preton pressure - if that is the mechanism - is in the order hyperthyroid $>$ euthyroid $>$ hypothyroid.

Evidence is discussed in section II-A that unsaturated cardiolipins contribute to proton retention at high $\Delta p$ under State 4 conditions, whereas the uncoupling protein in BAT leaks protons and disperses $\Delta p$. Increased amounts (cardiolipin/phospholipid) of abnormally saturated liver cardiolipins are associated with proton-leakiness and thermogenesis in mitochondria of hyperthyroid animals. The amount of uncoupling pro- 
tein is up-regulated by decreases in ambient temperatures and the resulting proton leakage is thermogenic. like the eftects of thyrotoxicosis. Hypothyroidism augments liver mitochondrial cardiolipin / phospholipid ratios and the cardiolipins are normally unsaturated; by their normal role in proton-retention, these cardiolipins should decrease proton leakage and thermogenesis; it is not clear if the phosphatidylcholines and phosphatidylethanolamines, winich also become less unsaturated, participate in proton-retention. It seems pertinent that chilled subjects secrete more thyroid hormone, and warmed subjects, less. Elevation of temperature also down-regulates amount of BAT mitochondrial uncoupling protein, proton-leakage and thermogenesis.

Thyroid-state affects State 3 respiration rates. Although cardiolipins are specitic for many of the regulated steps, mechanisnis of thyroid effects are not proven to be mediated by the known cardiolipin changes. State $3 \mathrm{Jp}$ is about $110\left(30^{\circ} \mathrm{C}\right)$ or $190 \mathrm{mV}$ $\left(37^{\circ} \mathrm{C}\right)$ in liver mitochondria from cuthyroids, and untreated or treated hypothyroids [120] (Table VIII). Effective proton conductance at $37^{\circ} \mathrm{C}$ is $5.84 \mathrm{ng}$ ion $\mathrm{H}^{+}$ $\mathrm{min}^{-1} \mathrm{mg}^{-1} \mathrm{mV}^{-1}$ in mitochondria from euthyroids and half that in hypothyroids. The proton routes initiated or augmented in mitochondria of hypothyruid rats by induction of State 3, namely the ADP/ATP carrier, ATP-synthase, $\mathbf{P}_{\mathrm{i}} / \mathrm{H}^{+}$symport, dicarboxylate carrier, cytochrome $b c_{1}$ and cytochrome oxidase, would be involved in these proton conductances, since the proton leak disappears in State 3.

The ADP/ATP carrier activity is sensitive to effects of altered thyroid states on: (i) the membrane environment of the carrier; (ii) am.ounts and exchangeability of ADP + ATP in the matrix; (iii) ratios of [free ATP]/[free ADP] in matrix and cytosol; and pcrhaps (iv) the proporticns of $\Delta \mathrm{pH}$ and $\Delta \psi$ (see above; this carrier discharges $\Delta \psi$ [155]). All these factors determine the carrier rate and, together with rates of other regulators, its $C_{i}$ contribution to State 3 control. (i) An altered membrane environment of the ADP/ATP carrier in liver mitochondria from hypothyroid rats is indicated by the linearity of the Arrhenius profile $\left(0-37^{\circ} \mathrm{C}\right)$ of external ADP translocation [281]: the profile in normals inflects sharply at $17^{\circ} \mathrm{C}$. As a result, at $0^{\circ} \mathrm{C}$ (a usual temperature for assay) hypothyroid mitochondria translocate external ADP at a rate $66 \%$ less than normal but at $25^{\circ} \mathrm{C}$ the rate is normal. The abnormal thermotropic properties are typical of effects of altered membrane lipids. (ii) Content of ADP + ATP in liver mitochondria of hypothyroid rats is $60 \%$ greater than in normals, and pyridine nucleotides are $40 \%$ above normal [281,341]. Of the $13.8 \mathrm{nmol}$ of ATP + ADP per mg proiein, hypothyroids exchange only the normal content of $9.6 \mathrm{nmol} / \mathrm{mg}$ via the ADP/ATP carrier $[281,586]$. A similar compartmentation occurs when ATP + ADP accumulates after glucagon treatment [255]. One LT4-injection corrects, in 3 days. the nucleotide compartmentation and temperature-late relationship. Accumulation of ATP can proceed through an $A T P-M g / P$, carricr that moves ATP passively down a concentration gradiont, but that would not account for abnormal co partmentation [15]: loaded nucleotidc. uxchange cor., letely, as shown by the linearity of adenine nucleotide translocase velocity with matrix [ATP + ADP] even at high concentrations.

(iii) The ATP/ADP ratio in the matrix of liver mitochondria from hypothyroid rats is subnormal [281] because ADP is phosphorylated slowly [99]; the ratio in the cytoplasm is high because ATP is utilized slowly (e.j., Ref. 165). Increased matrix ATP + ADP and AIJP/ATP ratio should accelerate translocation of external ADP, but the compartmentation leaves the abnurmal lipid dependency of the carrier in hypothyroid mitochondria to account for the slowed transport.

Claims that an identity of the ADP/ATP carrier with a rat liver mitochondrial T3-receptor accounts for in vitro and in vivo hormonal acceleration of State 3 respiration [630] are difficult to accept conceptually [245.284] and are contradicted experimentally [530].

The cardiolipin-specific sartiers of monocarboxylates, dicarboxylates and tricarboxylates. as well as acylcarnitines (palmitoylcarnitine transferases plus (acyl) carnitine translocase), are all thyroid-responsive [284] (Table I). In heart mitochondria of hyperthyroid rats (5-day T3-treatment), as compared with euthyroids, the pyruvate carrier $V_{\max }$ at $10^{\circ} \mathrm{C}$ is $70 \%$ higher, $K_{m}$ for pyruvatc is unchanged, oxidation of pyruvate in State 3 at $25^{\circ} \mathrm{C}$ is accelerated $40 \%$; cardiolipin/phospholipid is $50 \%$ greater but fatty acyl composition is not reported [494]. Cardiolipin per phospholipid ratios also rise in liver mitochondria, where cardiolipin 18:2 acyls are extensively replaced with saturated fatty acyls [563] (Table III). ihe tricarboxylate carrier in liver mitochondria does not seem to have been tested for State 3 regulation; its $V_{\text {mix }}$ at $4^{\circ} \mathrm{C}$ increases $62 \%$ in hyperthyroid rats, $K_{m}$ for citrate remains unchanged, and activity increases across th: range of $1-27^{\circ} \mathrm{C}$ while transition temperatures in A rhenius profiles shift from $18^{\circ} \mathrm{C} \rightarrow 13^{\circ} \mathrm{C} 1495$ ]. Mitochondrial $\triangle \mathrm{pH}$ remains at 55 $\mathrm{mV}$, and carrier amount also stays constant, suggesting that the hormune activates carrier through either increased general membrane fluidity or specific interactions between cardiolipins and carrier.

Liver mitochondria from hyperthyroid rats ( 5 days of T3) take up or exchange $P_{i} 50 \%$ faster than normals, as measured by $V_{\max }$ at $0^{\circ} \mathrm{C}$ [497]. The amount of $P_{i}$-carrier, the $K_{m}$ for $P_{i}$, the initial intramitochondrial $\left[P_{i}\right]$. and the $\Delta \mathrm{pH}$, are all close to normal. Paradies and Ruggiero [497] conclude that the increase in cardiolipin/phospholipid ratio accelerates $\boldsymbol{P}_{i}$-carrier activity, which may contribute to an increased mitochon. 
drial State 3 respiration because $P_{\text {, transport is ohliga- }}$ tory for malate, citra!c and $\alpha$-ketoglutarate transport. and closely linked to ADP and $\mathrm{Ca}^{2}$ " trailsport. However, the great capacity of the $P_{i}$-caricer usually $\mathrm{pr}:$ cludes a regulatory role. Selwyn [S88] cites a report of Gainutdinov et al. that thyroid hirmone treatmetil increases a heat-stable, cytoplasmic factor that accelerates equivalent proton influx along the $\mathrm{P}_{\mathrm{i}} / \mathrm{OH}$ ail. tiporter portion of a $P_{i}$ cycle that includes a $\mathbf{p H}$-dependent anion-conducting channel in liver and heart mituchondrial membranes.

The ATP-synthase in hcart is thyroid-dependent in situ and in vivo [353], and is the major regulator of State 3 in adult and fetal heart mitochondria. The ATP-synthase in adult liver mitochondria is also thyroid- and cardiolipin-dependent, hut dex; not regulate (Tahle I). In skeletal muscle, ATP takes up *P, mo*e slowly in thyrotoxics than in normals [334]. Ernster et al. [174] suggest that the embedment of the protonophoric $F_{0}$-ATPase in cardiolipins is necessary for ATPase function. A role for an abnormally conductive ATP-synthase $\left(F_{0}\right.$-leak) in the augmented portion of State 4 proton leakage in liver mitochondria from hyperthyroid rats $[298-300]$ is disputed (sec secti.n IV-C.2.d).

Insulin: cardiolipin fatty acyl distributions and cardiolipin/phospholipid ratios are normal in renal cor:ex total phospholipids of rats after 2 months of strepto totocin-induced diabetes [107] (Table III). Cardiolipin/ phospholipid quotients in liver mitochondria are normal in 4 - to 10 -week diabetic rats $[68,332,676]$ but slightly lowered in skeletal muscle mitochondria from chronically diabetic human patients [254]. Thus, fiom these few studies it appears that cardiolipins do not participate in the better-documented changes in polyunsaturated phospholipids from liver, hear: kiclncy and testis, and from liver mitochondria or microsones, that are reported in diabetic rats 2-70 cays after injection of alloxan or streptozotocin [61.6\$.107.164. 181,286.293,310,675j: $18: 2(n-6)$ and $\left.22: u_{i i}-3\right)$ acyl contents increase, $20: 4$ acyis are depleted. These fatty acyl changes arc seen in phosphatidylcholines and phosphatidylethanolamines, but only kidney cardiolipins have been analyzed [107]. If the normal kidney cardiolipins in diabetics indicate that liver cardiolipins are also normal (additional reasons are presented here to think they are), lipid-dependent abnormalities of mitochondrial function in diabetics are effects of altered fatty acyl compositions of phosphatidylcholines and phosphatidylethanolamines. Altered cardiolipins affect function differently.

Fatty acyl changes in lipids of diabetic subjects can be attributed to usual mechanisms: the fatty acyls of mitochondrial phospholipids are replaced in part by incoming fatty acyls, in this case mobilized from depot fat. Release of the hormone-seusitive lipase activity from insulin-inhibition in experimentally-induced diabetes mellitus rapidly mobilizes frec fatty acids from white adipose tissue triacylglycerols into plasma. Triacylglycerol fatty acyls include approx. $25 \%$ 16:0, 40\% $18: 1,25 \% 18: 2(n-6)$, and no $20: 4(n-6)$ acyls, in fasted rats [9] and in 3-week diabetic rats [310]. Albumin-bound fatty acids in plasma exchange with tissue falty acyls.

When diabetes is indused by alloxan injection, free fatty acid content of rat liver mitochondria increases linearly from $5 \mu \mathrm{g}$ (mg protein) $)^{-1}$ at $t=0$, at a rate of about $0.25 \mu \mathrm{g} \mathrm{mg}^{-1} \mathrm{~h}^{-1}$, to a level of $18 \mu \mathrm{g} \mathrm{mg}{ }^{-1}$ at $48 \mathrm{~h}$; no fatty acid composition was presented [419] but one can be inferred. Diabetes also increasis plasma [triacy!gi cercils] 4-fold; the liver esterifies free intty ar: iss. fur: her desaturates some, and incorporates both si:uriticd and unsaturated fatty acyls into triacylglycerols and phospholipids that are rel-ased to plasna. Normally, rlasma triacylglycerols coniain ahout 35\% $16: 0,40 \% 18: 1$ and $7 \% 18: 2$ acyls, which indicates that fat partly retains stored essential $18: 2(n-6)$ acyls. In diabetic rats $21-30$ days after streptozotocin administration, flasma triacylglycerol $18: 2$ acyl content is $24 \%$ at the expense of $16: 0$ and $18: 1$ acyls; plasma phospholipids contain twice the normal amount of $18: 2$ acyls and less than normal $20: 4$ acyls $[310,724]$; erythrocyte membrine phosphatidylcholines and phosphatidylethanolamines, which nurmally exchange with plasma lipids, mirror the high-18:2, low-20:4 acyl pattern in diabetics.

A high-18:2, low-20:4 acyl pattern in both mitochondrial and microsomal phosphatidylcholines and phosphatidylethanolamines, together with normal cardiolipins. occurs in mitochondria from hypothyroid rats, and is ascribed to observed decreases in hepatic $\mathbf{3 6}$ and 15-desaturations (see Ref. 284); fatty acid mobilization from triacylglycerols is suppressed in hypothyroidism. In contrast, fasting rats and refeeding them carbohydrates [9], or injecting hypothyroid rats with T3 [284], evokes newly biosynthesized saturated and monounsaturated fatty acious from lipogenic tissues, that partly replace liver organclle membrane fatty acyls. Most of the 18:2 acyls are displaced in cardiolipins from tiyperthyroid rats (Table III) but any contribution of stored fatty acyls in the depleted triacylglycerols is uncertain.

Thus, the augmented free fatty acids in liver mitochondria from diabetic rats wou!d have a high proportion of $18: 2$ acyls. The fatty acid composition of these mitochondrial phospholipids reflects, first the influx of 18:2 acyls; secondly the preservation of $18: 2$ acyls through the known defect in hepatic 16-desaturation $[196,204,205,428]$ that normally limits the conversion of $18: 2(n-6) \rightarrow 20: 4(n-6)$ acyls; and thirdly, influx of 16:0 and 18:1 acyls (the slowed 19-desaturation in diabetics $[204,317]$ is not accompanied by lowered $18: 1$ 
acyl contents in most studies). High $18: 2$ concentrations would protect the normal high $18: 2(n-6)$ acyl contents of cardiolipıns. Improved mobilization of stored 18:2(n-6) acyls and subsequent protection against desaturation may account for observations that an EFA-deficient diet depletes liver and heart phospholipid ( $n-6)$-unsaturated fatty acyl groups less in diabetic rats than in control animais [544]. The desaturation defect and the excess of $16: 0+18: 1$ acyls would remove $20: 4$ acyls where they normally abound. i.e. phosphatidyicholines and phosphatidylethanolamines. However, although the hepatic desaturative conversion of $19: 2(n-3) \rightarrow 22: 6(n-3)$ acyls (lab diets contain no 22:6) slows as early as 2 days after alloxan injection [286], 22:6 acyl conients increase in mitochondria from diabetic rats - probably because phosphatidylcholines and phosphatidylethanolamines avidly retain $22: 6$ acyls. as shown by their 2.3 -fold accumulation while 18:2 content is halved, in heart lipids of rats starved 7 days [727].

Altered mitochondrial membrane phospholipids in diabetic rats are accompanied by several changes. (i) Heart mitochondria and sarcoplasmic reticulum are enlarged in situ [534], which indicates that preparation artifact, \&u not account for the biochemical lesions. (ii) Activity of mitochondrial $\boldsymbol{\beta}$-hydroxybutyrate dehydrogenase decreases because amount of enzyme diminishes [103]; activity responds abnormally to temperature changes because the enzyme binds phosphatidylchoiines specifically and diabetic phosphatidylcholines have abnormal fatty acyl profiles [676].

(iii) Liver mitochondria from 2- to 3-day diabetic rats respire at normal rates at $37^{\circ} \mathrm{C}$ in both State 4 and State 3 [286]; State 4 respiration is normal at $30^{\circ} \mathrm{C}$ $[259,499,675]$ but $25-45 \%$ faster than controls at 23 $25^{\circ} \mathrm{C}[259,420]$. At $30^{\circ} \mathrm{C}$ Statc 3 respiration together with ADP/ATP exchange rate slows by $45 \%$ [388]: uncoupling is reported [252]; skeletal muscle mitochondria from human chronic diabetics oxidize slowly in both State 3 and State 4 [254.397]. These data may rifect abnormal thermotropic ploperties seen in 2-day ciabetics [286]: Arrhenius plots of State 4 rates are typically linear between 15 and $37^{\circ} \mathrm{C}$ [289] but $E_{\mathrm{a}}$ for diabetic mitochondria is $48 \mathrm{~kJ} / \mathrm{mol}$, for contruls, 64 $\mathrm{kJ} / \mathrm{mol}$. The ratio of State 4 rate (proton leakage) in diabetics/controls rises as temperature is decreased: 1.3 at $37^{\circ} \mathrm{C}$ and 2.2 at $15^{\circ} \mathrm{C}$. Increasing proton leakage uncouples; the ADP/O quotient diminishes as temperature is depressed. This unusual thermotropic property is associated with the abnormal phosphatidylcholines and phosphatidylethanolamines, and presumably normal cardiolinins.

(iv) Activity ci aniusi transporters alters, as shown by measurements of specific activity and total activity of cardiolipin-dependent transporters (see section II-B) extracted in the presence of beef heart cardiolipin and reconstituted in soy hean phosphatidylcholines [348]: dicarloxylate carrier increases, as does pyruvate carricr after 7 days; phosphate carricr does not change: citrate carrier decreases proneressively from 1 to 8 weeks after induction of diabetes. Intact mitochondra transport citrate $\left(8^{\circ} \mathrm{C}\right)$ at subnormal rates [95]. Slowed adenine nucleotide transport $\left(25^{\circ} \mathrm{C}\right)$ accompanies decreased State 3 respiration [388). Transport of fatty acyl-CoA molecules from cytoplasm to mitochondrial matrix. and thereby $\beta$-oxidation, is accelerated. The transporter set includes mitochondrial outer-face carnitine palmitoyltransferase $\rightarrow$ (acyl)carnitine translocase $\rightarrow$ inner-face carnitine palmitoyltransferase and is cardiolipin-dependent (see Ref. 284) and thought to be oriented through cardiolipin-binding [470). 10 days after rats are niade diabetic. outer-face and inner-face carnitine paimitoyltransferasc $V_{\max }$ doubles, in association with increased membrane fluidity as measured by a nonpolar-lipid probe: Arrhenius profiles of external-enzyme activity are almost linear in diabetics and controls, and converge toward $40^{\circ} \mathrm{C}$ (like plots of State 4 respiration; see above), while plots of inner-enzyme activity inflect similarly in control and diabetic preparations [50]. Outercnzyme activity $\left(37^{\circ} \mathrm{C}\right)$ incieases 2.5 -fold gradually from $48-120 \mathrm{~h}$ after insulin deprivation [222]. Activity of the liver mitochondrial (acyl)carnitine translocase at (r) is $80 \%$ greater in diabetics than in controls, but the cause is an increase in mituchondrial matrix [carnitine] in the diabetics [501]. The inconsistency of timing and direction seems to eliminate cardiolipin-dependency as the common mechanism for all these chinges in transporters.

Adrenergic agents: in rats injected daily for 2 weeks with norepinephrine ( $\beta_{1}$-agonist), most of the $18: 2$ acyls in heart lipid phosphatidylcholines and phosphatidylethanolamines (obtained at 2 months) are replaced by $20: 4(n-6)$ and $22:(n-3)$ acyls $[169,170]$. The cardiolipin fatty acyl composition and cardiolipin/ phospholipid ratios are not affected (Table 1II). be! amounts of triacylglycerols are halved. Such rats are subject to acute myocardial necrosis when stressed by catecholamine administration. In cardiac lipids from rats ireated with isoproterenol, a $\beta_{1} \beta_{2}$-agonist with similar sensitizing effects, 22:6 acyls partly replace $18: 2$ and $20: 4$ acyls [23j]. Miochondrial function was not reported.

Growth hormone: Maddaiah and coworkers [108110,402] describe mitochondrial phospholipids and respiration in hypophysectonized rats, and the effects of treatment with growth hormone and T4. In liver mitochondria fror: bypcuphysectomized rats, ine cardiolipin/phospholipid quotient is $26 \%$ less than in normals [110]. The fatty acyls of the cardiolipins are slightly repleted in 18:2 and lower in 18:1 contents; phosphatidylcholines and phosphatidylethanolamines contain less than normal proportions of $20: 4(n-6)$ and 
$22: 6(n-3)$ acyls, and phosphatidylethanolamines susprisingl: have almost no $18: 2$ acyls. Seven datly injections of growth, hormone raise cardiolipin/phospholipid $62 \%$ and normalize fatty acyl contents of all phospholipids. Liver mitochondrial respiration (glutilmate/malate. $310(\mathrm{C})$ slows by about $35 \%$ in both State 4 and State 3. and 7 daily growth hormone treatments do not correct respiraltory rates, hut 7 days of T4 raises rales 114\%; and 143\%, 10 levels well anove normal [108]. The discrepancy between growth-hormone-induced correction of phospholipid abnormalities and the persistence of respiratory changes may only reflect the temperature and time chosen for measurements. Arrhenius profiles of the State 3 rates in these 7-daytreated animals diverge from normals at the lower range of lemperatures. One injection of growth hormone accelerates Statc $\left.3(30)^{\circ} \mathrm{C}\right)$ in $12 \mathrm{~h}$ and alters fatty acyls of total phospholipids in $4 \mathrm{~h}$. suiggesting that the fatty acyl changes mediate at least part of the early effect on State 3 [4(1)2].

Mitoxhondrial phospholipids from hearts of hypophysectomized rats have normal proportions of cardiolipins, and normal fatty acyl compositions of total lipids [I(Y)]: no analysess of fatty atcyl contents of separated phospholipids were shown. A week of growith hormone injections increases total lipid 20:4 and deereases 22:h acyl contents. while $T 4$ has no effects thereon. Heart mitochondria from hypophysectomized rats respitc faster than controls in Statc 3 and Statc 4 at temperatures atove $20-25^{\circ} \mathrm{C}$, and slower at temperatures below that range, i.e. the Arrhenius plots cross. Hypophysectomy lincarizes the State 4 profile $(r=$ $-(1.978)$. and T4-treatment even more $(r=-0.997)$. 10 the degree seen in normal rat liver mitochondria [2ख9]. Some of the lipid-dependent effects of hypophysectomy on mitochondrial respiration appear to be mediated through the hypothyrolusm (sece section IV.C.2.d) of TSH-deficiency.

\section{IV.C.3. Localized ischemia and reperfusion}

Lecalized tissue ischemia. produced hy blocking arterial supply of oxygen. progressively removes a small portion of total phospholipids, mainly in the eurdiolipin fraction. from animal heart [59.3] and from milochondria of heart [668.669] and kidney [61.5] hut not liver [44(1)]. The cardiolipins remaining in pig heart after 4 ) min of ischemia (94\% of control) have normal fatty acyl compositions (Table III), as do the other phospholipids [593]. Ischemic skeletal muscles of rats show no depletion of cardiolipins at $4 \mathrm{~h}$; when they receive uxigen through reperfusion for $2 \mathrm{~h}$, cardiolipin contents fall by $45 \%$ while phosphatidylcholines and phosphatıdylethanolamines decrease by only $13 \%$ [621]. Cardivipin fatty acyl compositions were not reported. but the loss of cardiolipins was ascribed (1) their peroxidation by frec radicals produced from $\mathrm{O}_{2}$ metaholism. and the susceptihility of cardiolipins because of their unusually high content of unsaturated honds". Actually, the unsaturation index of cardiolipins in heart and muscle is usually less than that of the phorphatidylethanolamines that have high $22:(x n-3)$ acyl contents, hut alinost all fatty acyls of cardiolipins have two or ane unsaturated bonds. Presumably. peroxidation of fatty acyis potentiates removal of the headgroups of cardiolipins through the action of phospholipases $C$ and/or $D$.

The oxygen free-radicals preduced by ischemia/ repertusion appear to damage muscle. heart, liver. kidney and intestin " tissues with cardiolipins that have high 18:2+18:1 acyl contents: inhibiting $\mathrm{O}_{2}^{-}$production or scavenging $O_{2}^{-}$ameliorates damage. Ischemic damage exhibits as decreased mitechondrial fine structure [440.477] and diminished State 3 respiration with little change $[4,39,440,6(1) 4]$ or a rise $[328]$ in State 4 respiration. Lecause $J d$ and $J \mathrm{pH}$ in rat liver mitochondria remain normal $[13,22]$. the maintenance of normal State 4 respiration means that the apparent $C_{\mathrm{N}^{\prime \prime}}$ is decreased. The decrease in State 3 rate decrease is then due to altered $c$, contributions, which might include the observed loss of CoO [173] and cylechrorice wa, $(\$ 41))$ and decreased activities of NADH-ubiquinone reductase [134] and ADP/ATP carrier [6(6)4] but not the increased mitochondrial $\mathrm{Ca}^{2+}$ which was thought to dissipatc $1 p$ [1.34]. Since simple ischemia depletes mitochondrial cardiolipins only slightly and leaves the remaining heart cardiolipins with normial fatty acyls, the cardiolipins do not appear to be involved in the depression of State ? respiration. Cardiolipins may be involved in the mors severe changes during reperfusion: the oxidant activity of $\mathrm{O}_{3}$ added to cardiolipin-phosphatidylcholine-cytochrome $c$ vesicles abolishes cardiolipin-cylochrome $c$ binding [621]: ischemia depresses cytochrome aa $V_{\text {max }}$ in skeletal muscle mitochondria by $-28 \%$, and subsequent reperfusion lowers $l_{\text {nus }}$ by $-62 r^{\prime}$ : and an oriented cytochrome aa ${ }_{3}$-cardiolipin-cytochrome c complex may transfer electrons [678]. And cardiolipins bind the ADP/ATP carrier (section 1I-B). It should be noted that despite the prolonged ischemia produced by local vasisconstriction in skeletal muscles of diving seals, which conserves oxygen [579], the reperfused muscles are not apparently damaged.

\section{IV-C.4. Cultur i' cells: animal, yeast, plant}

Several animal cell lines in culture require addition of an essential $(n-6)$-polyunsaturated fatty acyl to keep mituchondrial oxidative phosphorylation coupled. Such cells have not usually been analysed for cardiolipin / phospholipid or fatty acyl/cardiolipin compositions (except for transformed lines), but data on their total phospholipids or total lipids suggest that cardiolipins are involved. Rat-heart cells cultured in a tat- 
free medium contain total lipids that are depleted of 18:2 and 20:4 acyls and repleted with 18:0 acyls. The cells stop growing and heating: oxidative phosphorylation is uncoupled, with decreased $A D P / O$ and respiratory control ratios (250.2(t)] but no State 4 and State 3 rates were shown. Added $18: 2$ or $20: 4$ fatty acid raises total lipid $18: 2+20: 4$ acyl conients and partly removes 18:() acyls, couples oxidative phosphorylation. but dees not restore growth or contractility. Heart cells from neonital rats grow better in serum than in added $18: 2$ acid, contain significant amuunts of $22:(x n-3)$ fatty acyls. and have cardiolipin P per total phospholipid $\mathbf{P}$ ratios about !nice those in adult or neonatal rat-heart lipids: no fatty acyl/cardiolipin compositions were shown [551]. These cells are thought to retain much of the phospholipid fatty acyl composition of their mother tissue, and to metabolize rather than incorporate supplied fatty acids. unlike other cultured animal cells.

HeLa-S , cells also need $18: 2(n-6)$ or $20: \$(n-6)$ fatty acid for growth [2(K,207]. On $18: 2(n-6)$ fatty acid, their total lipid fatty acyl profiles run about $44 C^{\circ} ;$ saturated fatty acyls. $20 \%$ monounsaturated fatty acyls. $9 \% 18: 2(n-6)$ and $12 \% ; 20: 4(n-6)$ fatty acyl groups. When no $(n-6)$-unsaturated fatty acids are supplied. monounsaturated fatty acyl contents increase 50-106\%\% and 18:2 and 20:4 acyls almost disappear. Homogenates of deficient cells are uncoupled [206,207]. with little or no respiratory control and decreased ADP / O ratios, but without stated State 4 rates wr can not tell if the proton-leak increases. Either $18: 2$ or 20:4 acid supports growth, repletes 20:4 and 18:2 fatty atgls 3! least partly, and couples mitochondrial oxidative phosphordation: the fatty acyl/cardiolipin composition with added $2(1): 4$ acid would be interesting to sec.

Heart cells culiured ionger than a day or so, the HeLa-S, cell strain, endothelia! cells, skin fibroblasts. and many cultured ceii line; [024] do not comert accumulated $18: 2$ to $20: 4$. A number of normal diploid cell lines perform $J 6$-and $\mathbf{1 5}$.desaturations, and some transformed cells lack fatty acyl-CoA 19 - and 36 -desaturases [160).445]. Studies on the influence of transformation on biomembrane lipid contents and compositions of a variety of cell liries are aften contradicion, perhaps because phospholipid compositions depend on malignas:cy of the tumor and diet of the host.

Mitochondria from hepatomas or ascites tumor cells contain about $50 \%$ more total phospholipid/mg protein and half the cardiolipin $\mathbf{P}$ per total phospholipid $\mathbf{P}$ found in rat or mouse liver mitochondria [444j; hut see Ref. 536. Their total lipid fatty acyl compositions feature either lower [35] or higher [536] 18: I acyl contents. and there is also disagreement on increased saturated fa!ty acyls and decreased 18:2 and 20:4 acyls. Cardiolipin fatty acyl contents differ from the expected liver cardiolipin patterns more consistently. The cardiolipins of hepaloma $72 \times 8 C$ cells. as compared with the normal cardiolipins of the host liver. are severely depleted in $18: 2$ acyls and have 4.4-fold higher contents of $16: 0+18: 0$ acyls and twice more $18: 1$ acyls [722.743] (Table III). In contrast, the 18:1 fatty acyls of the total phospholipids and the resolved phosphatidylcholines, phosphatidylethanolamines, and phosphatidylinositols are partly depleted [536]. These deviations in fatty acyl compositions are associated with altered differential scanning calorimetry profiles of phospholipids extracted from various hepatomas $[185,636]$, increased proton leakage, and changed membrane-dependenoy of several enzymes and carriers, most strikingly the $F_{1}$-ATPase.

Mitochondria from tumor cells respire abnormally rapidly, espucially in rapidly growing cells; State 4 in slow-growing hepatomas is $25 \%$; faster than in host liver, and $120{ }^{\prime} ;$ more rapid in fast-growing :umors, where State 3 also increases slightly [504]; State 4 is 50-40)\% above normal and State 3 up to $50 \%$ faster (loose-coupling) in hepatoma 7777 [536], hepatic isletcell tumor [231] and in rats fed carcinogenic aminoazo dyes [18]. State 4 is normal in Ehrlich ascites tumor cells $[573,656]$; and State 4 is decreased while State 3 respiration increases in mouse hepatoma $98 / 15$

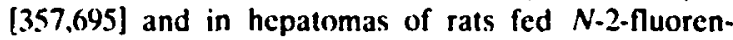
ylacetamide [28].

In those tumor mitochondria where the preton leak increases, ADP $/ O$ ratios are not lowered. However, abnormal function and thermotropic properties of the $F_{1}$-ATPase characterize the mitochondria of many hepatomas [444]. Although aduition of a protonophore or a $20^{\circ} \mathrm{C}$ increase in cimperature of assay accelerates ATPase activity 5- to 10 -fold in normal liver mitochondriu, the A TPase of hepatoma mituchondria responds minimally [5(14]. In one proposed mechanism for the ATP-synthase [462], the normally high $J p$ drives protions through the $F_{0}$ units to protonate the $F_{1}$-ATP-synthase so that it binds added ADP $+P_{i}$ more tightly and catalyzes a dehydration to form ATP. When \lrcorner $p=0$ because a protonophore is present, added ATP should bind to the unprotonated $F_{1}-A T P a s e$ and be hydrolyzed. Hepatoma $F_{1}$-ATPase under uncoupleractivated conditions either does not bind ATP or binds and does not hydrolyze. The loss of the normal ATP. ase heat activation suggests that the aberrant fatty acyls of the hepatoma phospholipids are involved. It may be pertinent that mitochondria from liver, kidney, heart (with 18:2-repleted cardiolipins), and skeletal muscle catalyze high uncoupler-activated ATPase activity. whereas mitochondria from brain and adrenal cortex have very low uncoupler-activated activity [505]. The cardiolipins of mitochondria of bovine adrenal cortex contain only $12 \% 18: 2$ and $65 \%$ saturated fatty acyls: cardiulipins of brain mitochondria have high 
polyunsaturated fatty acyl and low 18:2 acyl contents (Table III). In hyperthyroid rats, liver cardiolipins with low contents of $18: 2$ and high contents of saturated fatty acyls (like cardiolipins in turnors, may leak protons through the $F_{r}$-ATPase (see section IV-C.2.d); we do not know their uncoupler-activated ATPase activity.

Thus, some tumor cells grow independently of a supply of the EFA $18: 2(n-6)$ and its derivatives, and perhaps thereby acquirc rapid respiration that competes with host cells for substrates. Defective control mechanisms in the membranes of tumor mitochondria [684] and increased cytoplasmic $\left[P_{i}\right]$ and [ADP] have been connected with the characteristically rapid rate of aerobic glycolysis in tumor cells [685]. Indeed, a large supply of 18:2 fatty acid (or, lesi so, 18:1) kills Ehrlich ascites carcinoma cells [742] and a variety of carcinoma cells cultured in vitro (see $R \rightarrow f$. 742). Mice bearing the ascites tumor live longer when injected with linoleate, which elevates the 3\% 18:2 content in total phospholipids of ascites cells to $45 \%$. These findings suggest that the tumor cell abnormalities in fatty acyl content are primary adaptive changes.

Yeast mitochondria differ from animal mitochondria in several important respects. (i) $S$. cerecisiae cardiolipins have $96 \%$ monounsaturated fatty acyls and 4\% saturated fatty acyls [690] (Table III), animal mitochondria contain mainly $18: 2$ and $18: 1$ fatty acyls. (ii) Yeast mitochondrial State 3 respiration is regulated by cylochrome oxidase and/or the $P_{i}$ carrier and the proton leak (at low $\left[P_{i}\right]$ ) (Table $\left.I\right)$, in contrast to liver and heart milochondria. The yeast cytochrome oxidase is catalytically active when di-14:0-phosphatidylcholine replaces its firmly bound cardiolipins [690] (IIB). (iii) Yeast mitochondrial ATP-synthase activity is optimal at both pH 6.2 and pH 9.4 [262] while animal enzyme has the lower pit maximum and binds cardiolipins. (iv) Yeast mitociondria oxidize external NADH; the NADH dehydrogenase of animal mitochondria oxidizes only matrix $\mathrm{NADH}$, and requirc.s cardiolipins for reconstitution and binding to the matrix face of the inner membrane (see section 11-8). (v) Yeast mitochondria do not $\beta$-oxidize fatty acyl-CoAs but only incorporate the fatty acyls for structure of membranes [352]. The palmitoylcarnitine acyltransferase in animal mitochondria, which regulates $\boldsymbol{\beta}$-oxidation, has cardiolipin requirements like the NADH dehydrogenase for positioning toward the matrix (see section II-B). (vi) Yeast mitochondrial inner membranes contain sterols [49]: sterols in animal mitochondria are considered by some to be a sign of contamination with endoplasmic reticulum. (vii) Yeast mitochondria biusynthesize more phospholipids autonomously. While both yeast and animal mitochondria synthesize all the cell's cardiolipins and phosphatidylglycerol. the yeast mitochondria can also form considerable an:ounts of phosphatidylserine, phosphatidylinositol ans phosphatidylethanolamine: phosphatidylcholine comes from enzymes in the endoplasmic reticulum in yeasts $[1 \mathrm{i}: j$ anci animai cells. Jakovcic et al. [324] conclude that high cardiolipin/ phospholipid ratios characterize fully developed mitochondria of both yeasts and mammals.

The cardiolipin content of yeasts varies with species, genetic lesions [226], growth conditions (e.g., carbon source, temperature, $\left[\mathrm{O}_{2}\right]$, [inositol]) [410], and the degree of development of the mitochondrial inner membrane [325]. Stationary $S$. cerevisiue with glucose or galactose + lactate as carbon source contain more cardiclipin and have better developed mitochondria than repressed log-phase cells; so do aerobic cells versus anaetubic cells.

The phospholipids of many wild yeast strains contain $(n-6)$ - and ( $n-3)$-polyunsaturated fatty acyls, indicating the presence of $\Delta 12$ - and $\Delta 15$-desaturation systems. Some yeasts, particularly the Saccharamyces, contain only monounsaturated fatty acyls: 14:1, 16:1, and 18:1 [175,345]. Saturated fatty acyls are important in membrane; of a double-mutant strain of $S$. cerel'isiae defective in both fatty acid synthetase and 'desaturase [175]. Optimal growth stringently requires saturated fatty acids with 14-16 carbons, plus an unsaturated fatty acid. With any given saturated fatty acyl, unsaturated fatty acid effectiveness is in the order $18: 2(n-6)>18: 1(n-9)>18: 3(n-3)>20: 1(n-$ 9). The growth response is not explained by the degree of fatty acid incorporation into total phospholipids or changes in membrane fluidity as measured by ESR of a 12-doxyl-18:0 probe at $30^{\circ} \mathrm{C}$.

$S$. ceretisiae glown anaerobically or aerobically on media that contain glucose or galactose, ergosterol, Tween 80 (3\% 14:1, 13\% 16:1, 71\% 18:1, 13\% salurated fatty acyls), and no inositol, have total lipid fatty acyl crmpositions of about $\mathbf{8 0 \%}$ monounsaturated fatty acyls and $20 \%$ saturated fatty acyls [336]. The anaerobic cells contain about $0.9 \mathrm{mg}$ cardiolipin / $\mathrm{g}$ dry weight, and cardiolipin $P$ per total phospholipid $P=5 \%$. Aerobic cells contain $3.5 \mathrm{mg}$ cardiolipin / $\mathrm{B}$ dry weight, and cardiolipin $P$ per total phospholipid $P=12 \%$ - mitochondria proliferate shnut 4-fold (all the cardiolipins being mitochondrial in thrse yeasts; and more than double their cardiolipin/phospholipid ratios. Mitochondria from such aerobic yeasts ( $S$. cerecisiae strain Yeast Foam or N.C.Y.C. 239) oxidize added NADH through a second dehydrogenase on the outer aspect of the inner incmbrane (a matrix-sided dehydiogenase oxidizes endogenous NADH, as in animal mitochondria), which averts limitation of oxidative rates by a substrate carrier [421]; the cardiolipins may be involved (see section $11-\mathrm{B}$ ). At pH 6.5 or $6.7 .27^{\circ} \mathrm{C}$. State 3 respiration ( $\mathrm{ng}$ atom $0 \mathrm{~min}^{-1}$ ( $\mathrm{mg}$ protein) ${ }^{-1}$ ) with NADH is $740-800$, State 4 is 270 , State $3 u$ (CCCP) is 900 (State 3 (succinate) is 185) [688]). These mitochondria leak protons at a rate equivalent to that 
produced by $650 \mathrm{pmol}$ of $\mathrm{CCCP} / \mathrm{mg}$ protein as compared with $12 \mathrm{pmol}$ of $F C C P / \mathrm{mg}$ in rat liver mitochondria [228]; the 55-fold disparity is not quite accounted for by the 33-fold greater protonophore action of FCCP over CCCP [61]]. The rapid State 4 rate and the advent of a $C_{i}$ for proton leakage that partly regulates State 3 also suggest that yeast mitochondrial membranes leak protons faster than rat liver mitochondria; the different substrates and low $\mathrm{pH}$, and/or the fatty acyl composition of yeast cardiolipins, may be involved. Calculations from State 4 respiration (NADH) at $270 \mathrm{ng}$ atom $0 \mathrm{~min}^{-1} \mathrm{mg}^{-1} \cdot \mathrm{H}^{+} / \mathrm{O}=9$ [462]. and $\Delta p=170 \mathrm{mV}$ [421], give a value for $C_{\mathrm{M}^{H}} \cdot=14.3 \mathrm{ng}$ ion $\mathrm{H}^{+} \min ^{-1} \mathrm{mg}^{-1} \mathrm{mV}^{-1}$, almost 15 -fold the conductance calculated for rat liver mitochondria (see Table VIII).

Phospholipid subclasses in ycasts (as in mammals and bacteria) have distinctive and different fatty acyl compositions. In $S$. ceret isiae, cardiolipins are the most unsaturated $196 \% 16: 1+18: 1,4 \% 16: 0+14: 0$; see Table III) as compared with monounsaturated fatty acyl contents of $60 \%$ in phosphatidylinositols, $80 \%$ in phosphatidylethanolamines and $90 \%$ in phospharidylcholines [689]. Separate fatty acyl-CoA pools do not seem to account for the distinct compositions in yeast cardiolipins and phosphatidylethanolamines, which presumably draw on a mitochondrial pool; more likely, specific mitochondrial enzymes acylate deacylated cardiolipins to favor monounsaturated fatty acyl incorporation.

The mutant strain KD115 of Saccharomyces cererisiae, deficient in fatty acid desaturase and grown without inositol, can not biosynthesize monounsaturated fatty acyls but incorperates monounsaturated fatty acids from the medium into its mitochondria. Mitochondria with a monounsaturated fatty acyl content adjusted to about $80 \%$ of the total-lipid fatty acyls. and saturated fatty acyls to $20 \%$, oxidize $\alpha$-ketoglutarate $\left(\mathrm{pH} 6.5,30^{\circ} \mathrm{C}\right)$ at a rate of $40 \mathrm{ng}$ atom 0 $\mathrm{min}^{-1} \mathrm{mg}^{-1}$ in Stetc 4, 150 in State 3 with $\mathrm{P} / O$ ratios up to 2.5; cardiolipin / phospholipid ratio is $15.6 \%$ [262]. Rat liver mitochondrial values are comparable. In yeast mitochondria with asprox. $20 \%$ monounsaturatcd fatty acyls and $80 \%$ saturated fatty acyls, the cardiolipin/ phospholipid ratio rumains at $15.5 \%$ but State 4 respiration increases 3.4-fold and $\mathrm{P} / \mathrm{O}$ ratios are zero; the uncoupling results fruin increased permeability to protons, as shown by $\mathrm{H}^{+}$-pulsing [263]. Proton leakage also abolishes the energy-dependent uptake of $\mathrm{K}^{+}$but not the normal degree of membrane impermeability to $\mathrm{K}^{+}$. Raising the unsaturated fatty acyl sontent of the mitochondria to about $80 \%$ slows the proton leak, recouples oxidative phosphorylation and enables energy-supported $\mathrm{K}^{+}$-accumulation; either mono- or polyunsaturated fatty acids are effective. Recoupling is insensitive to chloramphenicol and cycloheximide, indicating that all the proteins for oxidative phosphorylation are in the unsaturated-fatty-acyl-deficient mitochondria and that the unsaturated-fatty-acyl-deficiency itself causes :eversible uncoupling. Free fatty acids are not responsible for the uncoupling: although free fatty acids can uncouple the recoupled mitochondria, free fatty acids do not accumulate in unsaturated fatty acyl-deficient mitochondria, and added albumin does not recouple (it does with added free fatty acids).

In yeasts, unsaturated-fatty-acyl-deficiency and/or reciprocal saturatid-fatty-acyl-repletion increases the permeability of the lipids of the inner mitochondrial membrane to protons, apparently enough to dissipate the drive for energy-linked reactions in oxidative phosphorylation; without measurements of $\Delta p$ we can not tell if proton conductance rises. In the liver mitochondria of EFA-deficient rats State $4 \Delta p$ is unchanged and so the increased rate of State 4 respiration should be due to increased proton conductance (see section II-A) - but we have no direct measurements by $\mathrm{H}^{+}$-pulsing. Nor are analyses available to show if the fatty acyl changes are localized or general among the phospholipid subclasses in yeast. Selective defects in cardiolipins do occur in mitochondria from livers of EFA-deficient animals (section IV-C.I.a) and of ethanol-ied rats (section IV.C.2.a). The unsaturated fatty acyl-deficient yeast cardiolipins might conceivably retain their monounsaturated fatty acyls, since a content of $96 \%$ monounsaturated fatty acyls and :a cardiolipin/phospholipid ratio of $15.5 \%$ could allow cardiolipins alone to contribute a ratio of $15 \%$ monounsaturated fatty acyls/phospholipids, and $20 \%$ monounsaturated fatty acyls are found. Cardiolipin analyses are needed.

Overall fatty acy! saturation varies directly with membrane proton permeability in mitochondria of yeasts and mammals. It should be noted that the proton permeability changes inversely with the degree of fatty acyl unsaturation; see section II-B for comparisons with model phospholipioj vesicles.

The effect of unsaturated fatty acyl-deficiency in yeasts that increases mitochondrial proton leakage differs from the effert of in'ssitol-deficiency that may include depressed mitochondrial respiratory capacity but certainly no increased proton leakage. A mutant $S$. carlsbergensis requires inositol for optimal growth [31,531]. Up to $90 \%$ of the inositol is in phospholipids. Lack of inositol is pleiotropic: it was originally said to diminish mitochondrial respiration, Krebs cycle activities, and contents of cytochromes and certain peptides [542]; lower total phospholip,ids per mg mituchondrial protein; lower phosphaiıdylinositol/total phospholipids (3.8\%); decrease cardiolipin/mg protein and increase cardiolipin/total phospholipids $(12.8 \%$-inositol vs. $9.2 \%$ + inositol). The los: acidic phosphatidylinositols are replaced by acidic cardiolipins, 
apparently because inositol depresics cardiolipin synthesis by inhibiting the mitochondrial pilosphatidylglycerolphosphate synthetase [226]. The replacing cardiolipins support mitochondrial function or development. Later studies found equal respiration, mitochondrial respiratory chain components, and mitorhondrial morphology in these ycasts grown with or without inositol $[209,492]$ but reported abnormal cell walls due to accumulation of glucans [209].

Phosphatidylinositols do not seem to he as specific as cardiolipins for mitochondrial function. Mammalian intracellular membrane total phospholipids have a phosphatidylinositol contert of about $8 \%$ [264]; in $S$. ceretisiae the phosphatidylinositol is $25 \%$ and cardiolipin is about $4 \%$ during anaerobic growth and $17 \%$ and $12 \%$, respectively, during growth $+\mathrm{O}_{2}[3336]$. In promitochondria, phosphatidylinositol/phospholipids $=25 \%$, cardiolipin $=9 \%$, when this yeast grows without added lipids, but the endoplasmic reticulum also has a high phosphatidylinositol/phospholipid ratio [493].

\section{Summary}

Evidence is discussed for roles of cardiolipins in oxidative phosphorylation mechanisms that regulate State 4 respiration by returning ejected protons across and over bacterial and mitochondrial membrane phospholipids, and that regulate State 3 respiration through the relative contributions of proteins that transport proions, electrons and/or metabolites.

The barrier properties of phospholipid bilayers support and regulate the slow proton leak that is the basis for State 4 respiration. Proton permeability is in the range $10^{-3}-10^{-4} \mathrm{~cm} \mathrm{~s} \mathrm{~s}^{-1}$ in mitochondria and in protein-free membranes formed from extracted mitochondrial phospholipids or flom stable synthetic phosphatidylcholines or phosphatidylethanolamines. The roles of cardiolipins in proton conductance in model phospholipid membrane systems need to be assessed in view of new findings by Hübner et al. [313]: saturated cardiolipins form bilayers whilst natural highly unsaturated cardiolipins form nonlamellar phases. Mitochondrial cardiolinins apparently participate in hilayers formed by phosphatidylcholines and phosphatidylethanolamines. It is not yet clear if cardiolipins themselves conduct protons back across the membrane according to their degree of fatty acyl saturation, and/or modulate proton conductance by phosphatidylcholines and phosphatidylethanolamines.

Mitochondrial cardioiipins, especially those with high 18:2 acyl contents, strongly bind many carrier and enzyme proteins that are involved in uxidative phosphorylation, some of which contribute to regulation of State 3 respiration. The role of cardiolipins in biomembrane protein function has ieen examined by measuring rutained phospholipids and phospholipid hinding in purified proteins, and by reconstituting delipidated proteins. The reconstitution criterion for the significance of cardiolipin-protein interactions has been catalytical activity: proton-pumping and multiprotein interactions have yet to be correlated. Some proteins. e.g. cytochrome $c$ oxidase are catalytically active when dimyristoylphosphatidylcholine replaces retained cardiolipins. Cardiolipin-protein interactions orient membrane proteins, matrix proteins, and on the outerface receptors, enzymes, and sime leader peptides for import; activate enzymes or keep them inactive unless the inner membrane is disrupted; and modulate formation of nonbilayer $H_{11}$-phases. The capacity of the proton-exchanging uncoupling protein to accelerate thermogenic respiration in brown adipose tissue mitochondria of cold-adapted animals is not apparently affected by the increased cardiolipin unsaturation; this protein seems to take over the protonophoric role of cardiolipins in other mitochondria.

Many in vivo influences that affect proton leakage and carrier rates selectively alter cardiolipins in amount per mituchondrial phospholipids. in fatty acyl composition and perhaps in sidedness; other mitochondrial membrane phospholipids respond less or not at all. Cardiolipins with high contents of 18:2 acyls. less monounsaturated and very few saturated fatty acyls normally occur in liver, heart and kidncy mitochondria, where State 4 respiration varies with thyroid hormone level: increase in proportions of normal cardiolipins, logether with a high-18:2. low-21):4 acyl pattern in liver mitorhondrial phosphatidylcholines and phosphatidylethanolamines. accompany slowed State 4 respiration in hypothyroidism. Cardiolipins with low $18: 2$ acyl contents are found in mitochondria of tissues that respire independently of thyroid state, e.g., brain. spleen, testis, adrenal cortex, brown adipose tissue; during cold-adaptation the 18:2 acyl content and unsaturation of brown adipose tissue mitochondria increase while State 4 respiration slows. Phospholipidleak accelerates when mitochondrial cardiolipin 18:2 acyls are replaced by saturated or monounsaturated acyls. as in mitochondria prepared from some tissues of animals that are in postgastrulation embryonic state, fetuses, hyperthyroid, essential fatty acid-deficient, erucate- or ethanol-fed: and from cold-adapted fish, unsaturated fatty acid-deficient yeasts, and hepatoma cells. Phospholipids including cardiolipins in plasma membranes of thermophilic eubacteria contain only saturated and branched fatty acyls and are stable at high temperatures but leak protons. Conversely, diets or culture media that replace cardiolipin 18:2 acyls with other unsaturated fatty acyls (e.g., $(n-3)$ unsaturated or 22:1(n-9)) without changing the low content of sat!rated fatty acyls, or that introduce a strange fatty acyl (linelaidoyl) into phosphatidyl- 
cholines and phosrhatidylethanolamines but not cardiolipins, do not increase mitochondrial proton lcakage. Saturated fatty acyls of cardiolipins thus seem to be associated with the proton leak.

\section{Acknowledgemints}

The constructive comments of the anonymous reviewer improved the balance between the bionhusical and biological portions of this review. I appreciate the help I have received from the following investigators: Drs. M.D. Brand and R.P. Hafner have advised me on effects of phosphate ions on proton leaks; Dr. R.L. Wolff has provided unpublished data on cardiolipin fatty acyl compositions; information and prepublication texts have been supplied by them and Drs. L. Ernster and C.-P. Lee, R. Hansford, M. Horrum, K. Hostetler. G. Paradies, M. Seewald and E. Shrago.

\section{References}

1 Aaronnmit. i.R. Johnston. A.M. and Martin. (C.E. (1983) Biochim. Birphys. Acta 713. 456-462.

2 Abramowitch. D.A.. Marsh. D. and Powell. G.L. (] $(9 \times 1)$ Biuchim. Biophys. Acta 1020, 34-42.

3 Adams, V., Busch. W., Schlegel. J., Walliman. T. and Brdiczka. D. (1949) Biochim. Biophys. Acta 981, 213-225.

4 Adkisson. H.D., IVth. Risener, F.S.. Jr., Zarrinkar, P.P.. Walli. M.D. Christic, W.W. and Wuthier. R.E. (|q9|) FASEB J. S. 344-353.

5 Adkision. H.D. IVih. Trancik, T.M. Zarrinkar. P.P. and Wuthier, R.E. (19\%(1) Orthop. Trans. 14, 423-\$24.

6 Alberts. B.. Bray, D., Lewis. J., Raff, M., Roherts, K. and Watson. J.D. (1983) Molecular Biology of the Cell. p. X14. Garland. New York.

7 Alfin-Slater. R.B. and Afterg(x)d. L. (1968) Physiol. Rev. 4X. $758-784$.

8 Allegrini, P.R. Pluschke, G. and Seelig. J. (1984) Binchemistry 23. 6452-645K.

y Allmann, D.W.. Huhbard, D.D. and Gibson. D.M. (1\%,5) 3. Lipid Res. 6, 63-74.

10 Aloia. R.C. and Raison, J.K. (1989) Biochim. Biophys. Acta 988. 12.3-1 th.

11 Alonss), T.L.. Bonini de Romanelli. J.C. and Bazán. N.G. (1982) Biochim. Bicophys. Acta 68 h, 145-151.

12 Alvager. T. and Balcavage. W.X. (1978) Age 1, 42-48.

13 Andersson. B.S.. Aw. T.Y. and Jones. D.J. (1987) Am. J. Physiol. 252. C349-C355.

14 Anel. A., Naval, J., González. B.. Torres, J.M., Mishal. Z., Uriel. 3. and Piñeiro. A. (1991)) Biochim. Biophys. Acta 1(444, 323-331.

is Aprille, J.R. (1986) FASEB J. 2, 2547-2556.

16 Aquila. H., Link. T.A. and Klingenterg. M. (1987) FEBS Lett. 212. 1 -9.

17 Arai. M., Gordon, E.R. and Lieber, C.S. (1944) Biochim. Biophys. Acta 797, 320-327.

18 Arcos, J.C., 'Tison, M.J., Gosch, H.H. and Fabian. J.A. (|quo| Cancer Res. 29, 1298-1306.

19 Ardail, D., Privat, J.-P., Egret-Charlier, M., Levrat. C., Lerme. F. and Louisot, P. (1990) J. Biol. Chem. 265. 18.797-1880)2.

20 Arthur. H. and Watcon. K. (1976) J. Bacteriol. 128. 56-68.

21 Auslander, W. and Junge. $w$. (1974) Biochim. Biophys. Acta $357,285-298$.

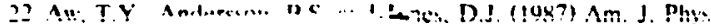
iiv) $252.0356-(36)$

23 Awisthi, Y.C., Bereancy. R. Rusichis. F.J. and Crane. F.L. $(\mid(6,4)$ Bicxhim. Bionply. Acta 1K4. 457 tox).

If Awiothi, Y.C., Chuang. T.F. Keenan. T.W. and Ci.lne. F.l. (1971) Biochim. Biophys. Acta 226. 42-52.

25 Bagpetto. L., Gautheron. D. and Godinot. C. (19K+) Arch. Bicchem. Biophys. 232. 6:70-67k.

26 Baht. H S. and Saggerwon. E.D. (148X) Biochem. J. 251, 553-557.

27 Bank. W., Argow. Z.. Leigh. J.S. and (hance, B. (1987) Ann. N.Y Acad. Sci. S(kx, $44 k-450$.

28 Banks. W.L.. Jr., Terz. J.J. and Higgins, E.S. (1971) Proc. Soc. Exp. Med. Biol. 138, 325-329.

29 Baraniska. J. and Whodawer. P. (1\%69) Comp. Biochem. Physiol. 28. $553-570$.

30) Beauwoit. B., Rigoulet, M. and Guèrin B. (1069) FEBS Lett. 244, 255-258.

31 Bednarz-Prashad, A.J. and Mize, (.E. (1978) Biochemistny 17. $4178-41 \% 5$

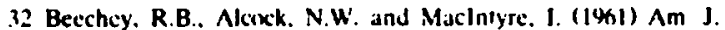
Physiol. 201, 11201-1122.

3.3 Beeler. D.A., Trable. D.H., Kyriakides, E.C. and Balint, J.A. (1970) Biochim. Biophys. Acta 21x, 112-123.

34 Behrens. W.A. and Himms-Hagen. J. (1977) J. Bicenerg. Biomembr. 9. 41-6.3.

35 Bergelson, L.D.. Dyatlowitskayi, I .V.. Torkhowkaya. T.J., Sorokina. L.B. and Gorkerva, N.P. (1970) Biochim. Biophys. Acta 210. 287-298.

36 Berrez. J.-M., Pattus. F. and Latruffe. N. (1985) Arch. Biochem. Biophys. 24.3, 62-69.

i7 Reyer, K. and Klingenberg. M. (1985) Biochemisty 24. 38213826.

38 Bever, R.E. (1\%,3) Fed. Proc. 22, k74-88.1.

39 Biarico, A.C. and Silva. J.E. (1987) J. Clin. Invest. 79. 295-300).

40) Bianco, A.C. and Silva, J.E. (1987) Endocrinology 120, 55-62.

41 Biran. I 1. Bartley, W.. Carter, C.W. and Renshaw. A. (1964) Bixchem. J. 93, 492-498.

42 Blair. R.J. and Weller. P.F. (1987) Mol. Bixchem. Parasitol. 25. 11-18.

43 Bligny. R. and Dowce. R. (19ख(1)) Biochim. Biophys. Acta 617. 254-263.

44 Bligny. R.. Rebeillé, F. and Douce, R. (1985) J. Biol. Chem. 261). 9166-9170.

45 Blomstrand, R. and Svenswon, L. (1474) Lipids 9. 771-780.

46 Boratman, J.B. (1959) Am. J. Physio'. 1\%. 983-946.

47 Buhnensack. R.. Küster. U. and Letko, G. (1942) Biøxhim. Bisphys. Acta 6\$0). 271-28(1).

48 Bonini de Romanelli. I.C.. Alonso. T.S. and Bazán, N.G. (1981) Bischim. Biophys. Acta 664. 561-571.

49 Boltema. C.K. and Parks, L.W. (1980)) Lipids 15. 987-992.

5(; Brady, L.J., Silverstein, L.J., Hoppel. C.L. and Brady, P.S. (1985) Biochem. J. 232, 445-45U.

51 Bramhall, J. (1987) Biuchemistry 2h, 2848-2855.

52 Bramhall, J., Mofmann, J., DeGuzman. R., Montestruque, S. and Schell, R. (1987) Bixchemistry 26. 6330-6341).

53 Brand. M.D. (1990) Bixchim. Biophys. Acta 1018. 128-133.

54 Brand. M.D., Hatner, R.P. and Brown. G.C. (1988) Biochem. J. 255. 535-539.

55 Brand. M.D. and Murphy. M.P. (1987) Biod. Zev. 62, 141-193.

56 Brautbar. M.D. and Altura. B.M. (1987) Alrrholism Clin Exp. Res. 11, 118-126.

57 Brdiczka. D., Kn'll), G., Riesinger, I., Weiler, U., Klug, G.. Benz. R. and Krause, J. (1946) Adv. Exp. Med. Biol. 194, 55-64.

58 Brenner. R.R. (1974) Mol. Cell. Biochem. 3, 41-52.

54 Brenner. R.R. (1977) Adv. Exp. Biol, Med. 83, 85-101.

60 Brenner. R.R. (1987) Prog. Lipid Res. 23, 69-96. 


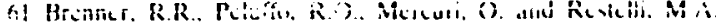
(146x) Am. J. Physicl. 215.637.69.

62 Buwn. (i.C. and Brand, M.:) (1966) Bickhem. J. 234, 75. 31

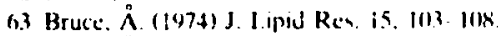

of Bruce. Aै (1974) J. Lipial Res. 15. 1149-11.3.

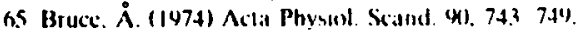

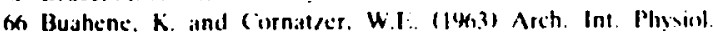
Bixchim. 71. I95 21H.

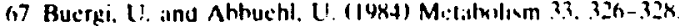

to Burke, J.P. and Fenton. M.R. (IYXo)) (irmp. Birchem. Phosiol. 9.313, $4(1)-\$ 12$

64 (afim, D.S. and Hubbeli, W.L. (1983) Biophys. J. 44. 49-57.

7) Caldwell. R.S. (1464) Comp. Bioxhem. Physwil. 31. 74-93.

71 Caldwell. R.S. and Vernterk. F. J. (1470) (amp. Birchem. Physiol. 24, 179- $\mid 91$.

72 Cannon. B., Alexwin, S. and Nedergitard, J. (19K2! Ann. N.Y. Acad. Sci. 3\%6. 41)-57.

73 Cunnon. B. and Nedergitard. J. (1985) Ewaty Bickehenr. 201. 110-164.

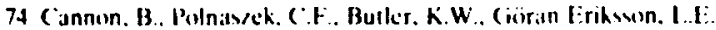
and Smith. I.C.P. (1275) Arich. Biochem. Biophys. 167, 505..518.

75 Capildi. R.A. (1)6s) Trend Buxhoin. Sci. 13. 1\$4-14k.

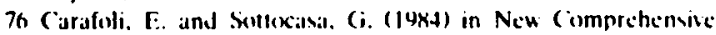

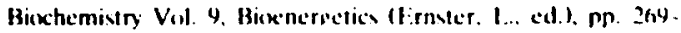
2x9. Elseviter. Amsterdam.

77 Cartunera. D. and Arsone. (i.F. (1986) Bischim. Biophys. Acta 94.3, 24.5-25.

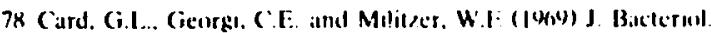
97. Irith-192.

79 (asiro. J.. Cintis. J.P. and (iu/minn. M. (| $44 \mid)$ Biekhem. Phar macivi, 41. 1487-1945.

No (avalier-Smith. T. (1967) Nalure 32h, 332-3.33.

81 Ciderbaum. A.l. Licter. (.S. and Ruhin. I: (1474) Arch. Biexhem. Biophys. 16.5. $56(x)-569$.

K2 (ievc. (i. (1987) Biechemisity 2h. h, 3) $15-6,311)$

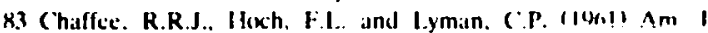
Physiol. 201. 24-32.

4. Chan. S.I. (198X) Ann. N.Y. Acind. Sci. 550. 207-222.

8.5 Chan, S.K. and Lenter. R.L. (1971)) Biexhim. Buphys, Actat 211 . $|x| 1|-| x \mid$.

the Chance. It ard Williams, (i.R. (1455) J biol. (hem. 217. 4(144424.

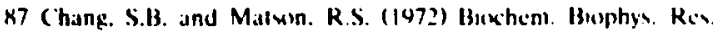
(iimmun. th, $1529-1535$

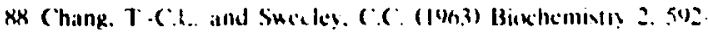
(x)

84 Chanman. D. and Leslic. K.B (1470) in Menobranes of Milochondria and (hloroplasts (Racker. E.. sd.). nn Ml-12h. Van Nostrand Rcinhold. Nek York.

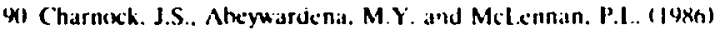
Ann. Nutr. Metah. 31), 39.3-416.

91 Chase. J.W. and Dauid. J.B. (1972) Dev. Biol. 27. 5(14-51K.

92 (heah. K.S. (19x+4) Bickhem. Six. Trans. 12. 35x-3

9.3 Cheah. K.S. and (heah. A.M. (IYXa1) in Memibrane Fluidity. Bicophysical Techniques and Cellulas Regulation (Kates. M. and Kuksis, A.. cds.). pp. $141-151$. Humana. (Clifton.

94 Cheah. K.S., Cheah. A.M. Fletcher, J.E. and Rosenherg. H. (IY84) Int. J. Biochem. 21, 913-4211.

95 Cheema-Dhadli. S. and Halperin. M.L.. (1973) (an. J. Bicchem. 51. 1542-1544.

\% Chen, J.C.. Warshaw, J.B. and Sanidi, D.R. (1972) J. (ell Physiol. \&(1. $\mid+1-1+4$.

97 Chen, Y.D.I. (1975) Fed. Proc. 34, 314.

98 Chen, Y.-D.I. and Hech, F.L. (1976) Arch. Biechem. Biuphys. 172. $: 11-744$

99 Chen. Y.-D.I. and Hech. F.L. (1977) Arch. Biexhem. Biophys. 181. $470 .+1,3$.

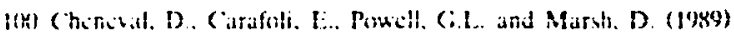
Eur. J. Biechem. 18h. $\$ 15-419$.

1111 Cherqui. (i.. (ador. M., Senatult. C. and Portet. R. (1979) Bickhim. Bionhws Acta 551 . 314--314.

1113 ('hiu. Y.J.1). and Richardum. A. (19xil) Exp. Gicrontol. is. $511-517$.

111.3 (hurchill, P. McIntyre. J.O., Vidal. J.C. and Fleischer, S. (198.3) Arch. Biuchem. Biwphys. 224. 659-670.

ll14 (icerr. T.J. and Sherman. W.R. (1471) Biochem. Biuphys. Res. cimmun. 43, 451-455.

los COandinin. M.T. (147X) J. Nutr. J11X. 27,3-2X1.

Ilk Clandinin. M.T. and Innis. S M. (198.3) Mech. Ageing Dev. 22. $2(15-20) x$.

1117 (lark. D.L. Hamel. F.(i. and Queener. S.F. (198.3) Lipids 18. (146-705.

IIK Clejan. S.. Collipp. P.J. and Maddaiah. V.T. (1480) Arch. Bitchem. Biophys. 2013, 744-752.

1119 C Kejan. S.. Jonas, F.. Collipp. P.J.. Fupler. I. and Maddadith, V.T. (1981) Bickhim. Biunhus. Acla 678. 2501-25t.

110 ( kejin. S. and Madddaiah. V.T. (148h) l.ipids 21. 677-683.

III (melik. S. and Fonvecal, E. (1974) Hopm-Sxyler's Z. Physiol. ('hem. 355, 19. 26 .

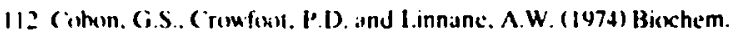
J. 1.44. 26.5 .27 .5 .

113 Cillocill. A. Nachhaur. J. and Viznais, P.M. (1971) Bixchim. Biuphys. Actat 249. 4t: 442.

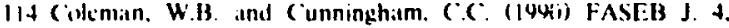
A 2274 .

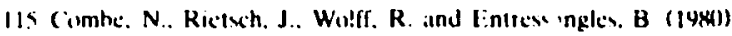
Ann. Nutr. Alim. 34. 3115-316.

116 (omle. J., Mä̈sterrena, B and (iautheren. I).C. (19\%h) Bicchim. Bionhys. Acla +14 . 27I-2X4.

117 (in). H.W. (1478) J. Neurcehen. 311. 1327-1334.

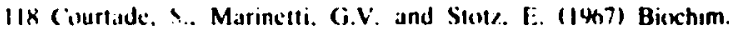
Biophys. Acta 1.37. 121-1.34.

119 (i) (Binl. 2(th. inl-2xu).

120 (respo-Armas, A. and Mouhras. J. (1987) Bickhem. J. 241. $057-6 n t)$

12: Cronan. J.1:.. Jr. (14hX) J. Bacteriol. 45. 21154-21k!

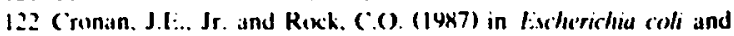
Salmomella nphrmarimm. Cellular and Molecular Biokgy (Neidhardt, I.C.. ed.), Volt. 1. pp. 474-497. Am. Soc. Microbiel.. Washing!: n

123 ('ronan. J.E.. Jr. and Vatgelos, R. (1972) Biechim. Biophys. Acta $265.25 \cdot$. nd 1 .

$12+$ Cillen, J.. Phillins. M.C. and Shipley, G.(i. (1471) Biaxhem. J. 125, 7.3K-742.

125 (ullis, P.K. De Kruijff. B.. Hopc. M.J.. Nayar. R. and Schmid. S.L. (lexull Can. J. Biexhem. 5x, l(ky)-11(x).

II culp. R.K., Lands, W.E.M.. I.ucihoi, B.R.. Pill. B. and Rom(un. J. (14X(1)) Prostaglandins 20). 11121-1(1)3].

127 Cunnanc. S.C.. Stiti. P.A.. Ganguli. S. and Armalrong. J.K. (1)(0)) (un. J. Animal Sci. 70. 251-254.

I2K Cunningham. C.C.. Filus. S.. Banticnti.. R.E. and Sn.h. P.1. (1482) Bickim. Biophys. Actit 712. 225-233.

124 Cunningham, C.C. and George, D.T. (1475) J. Biol. (hem. 251). $20136-2144$.

130 (unningham. C.C. and Sinthusek. G. (1974) Biochim. Biophys. Acta 550$)$. $150-153$.

1.31 Dale. M.P. and Robinson. N.C. (1488) Biuchemistny 27. 82708275.

1.32 Danforth. E.. Jr. and Burger. A. (194-4) (lin. Endocrinol. Metah. 13. $581-595$.

1.33 Danks. S.M. and Tribe, M.A. (1979) J. Thermal Biol. 4. 18.3-195.

1.34 Darlcr.-Usmar, V.M., Smith, D.R., OLealy. V.J., Sione. D., 
Hardy. D.L. and (Tark. J.B. (19y(x)) Binchem. Six. Trans. Ix. $526-52 x$.

135 Darnold. J.R.. Vorheck. M.L. and Marlin. A.P. (I) Ageing Dev. 53, 157-107.

136 Daum. (i. (1965) Bickhim. Biophys. Acta X22. 1-12

1.37 Dawid. I.B. and Blackler. A.W. (1472) Dev. Biol. 24, 152-1h1.

1.38 De Kruijf, B. and (ullis, P.R. (1980) Biochim. Biophys. Acta (x)2. 477-4x).

139 De Kruijff, B., Cullis, P.R.. Verkleij. A.J., Hope. M.J., Van Echteld. C.J.A. Taraschi. T.F., Van Hexogevest, P., Killian. J.A. Rietveld. A. and Van der Steen. A.T.M. (19K5) Prog. ProteinLipid Int. I. 89-142.

140 De Kruijff. B.. Nayar. R. and Cullis, P.R. (1982) Bickhim. Biophys. Acta 644. 47-52.

141 De Kruijft. B., Verkleij. A.J.. Van Echteld. C.J.A., Gerritsen, W.J.. Noordam. P.C.. Mombers. C., Rietveld. A., De Gier, J., Cullis. P.R.. Hope. M.J. and Nayar. R. $(|9 K|)$ in Internalimal Cell Biology 1981)-1981 (Schwelger. 11.G.. ed.), pp. 559-571. Springer Verlag, Berlin.

142 De Mendoza. P. and Cronan. J.E.. Jr. (148,3) Trends Biechem. Sci. 8, 49-5i

14.3 De Rosa, M. and Gambacona. A. (14kx) Prog. Lipid Res. 27. 153-175.

144 De Roxal. M., Gambacorta, A. and Gilinzzi. A. (1986) Microhiol. Rev. 50, 70)-8(1).

145 Deamer. D.W. (1987) J. Bisenerg. Binmembr. 19. 457-479.

146 Deamer. D.W. and Bramhall, J. (19X6) (hem. ?hys. Lipids 4). 167-18\%.

147 Deaver, O.E., Wander, R.C., McCiuser, R.H., Jr. and Berdanier, C.D. (1986) J. Nutr. 116, $1148-1155$.

148 Dest:mukh, D.R., Owen, O.E. and Patel, M.S (19*(1)) J. Neuruchem. 34. 1219-1224.

149 Deshmukh, D.R. and Patel. M.S. (1989i) Miccil. Agcing Dev. i., 75-81.

150) Desmeth, M. and Vandeputte-Poma. J. (1981) Lipids 16. 7(x)702.

151 Dewailly. P.. Nouvelot. A.. Sezille. G.. Fruchart. J.C . and Jaillard. J. (1978) Lipids 13. 3011-344.

152 Dewailly. P.. Sezille, G. Nouvelot. A.. Fruchart, J.C. and Jaillard, J. (1977) Lipids 12, 301-316.

153 Diwakaran, P. (197k) Experientia 34, 154)-1541.

154 Divakaran, P. and Venkataraman. A. (19;); J. Nutr. 1177,1621 1631.

155 Doussiere, J., Ligeti, E.. Brundolin. G. and Vignais, P.V. (1944) Biochim. Biophyc Acta 76h, 492-500.

156 Dow.Walsh. D.S.. Mahadevan. S.. Kramer. J.K.G. and Saucr. F.D. (1975) Biochim. Biophys. Acta 3\%. 125-132.

157 Drabkin. D.L. (195()) J. Biol. Chem. 182. 335-349.

158 Drees. $M$. and Beyer. K. (1988) Biochemistry 27. 8584-8541.

159 Dubinsky. W.. Kandrach, A. al,d Racker. E. (1979) in Membrane Bioenergetics (Lee, C.-P., Schatz, G. and Ernster. L., eds.) mo ?67-00. Artatson-Wesley, Reading.

160 Dunbar, L.M. and Bailey. J.M. (1975) J. Biot. Chem. 250, $1152-1153$.

161 Duszynski, J.. Grocen. A.K. Wanders, R.J.A., Vervourn, R C. and Tager, J.M. (1982) r.EBS Lett. 146, 262-266.

162 Dyatlovitskaya. E.V. Torkhowskaya, T.L. and Bergel'son, L.D. (199) Biochemistry (Biokhimiya) 34, 144-148.

163 Eble. K.S. and Cunningham. C.C (1987) Fed. Proc. 46. 1966.

164 Eck. M.G., Wynn. J.O. Carter, W.J. and Faas, F.H. (1979) Diabetes 28, 479-485.

165 Edelman, I.S. (1974) N. Eng. 1. Med. 290, 1303-13188.

166 Edman. K. and Ericson, I. (1487) Bixchem. J. 243, 575-578.

167 Elamrani. K. and Blume. A. (1983) Biochim. Biophys. Acta 727. 22-30.

168 Ellingson. J.S., Taraschi, T.F., Wu, A.. Zimmerman. R. and Rubin. E. (1988) Proc. Natl. Acad. Sci. USA 85, 3353-3357.

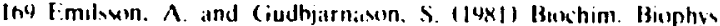
Acta hod. $82 \cdot-\mathrm{xN}^{2}$.

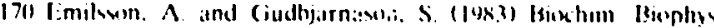
A(1.4 75i) 1-0

171 Ende. T. and Schitt. (;. (14XX) E:MBO) J. 7. 1153-115\%.

172 Eenster. L. and Kuylemsicrna, 13. (147(1) in Membranch of Mito chondria and (hloroplasts (Ratcker. 1.. ed.) pp. 172-212. Van Nostrand Reinhold. Now York

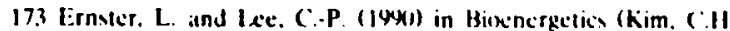
and ()zawa. T., eds.) pp. 451-th5. Plenum Prews. New York.

174 Ernster. L.. Sandri. Ci.. Hundal. T.. Cirlswon. C. anú Nordenbrand. K. (1977) in Structure and Function of Energy-Transducing Membranes (Van Dam, K. and Van (ielder, B.F.. eds.), pp. 2(Y)-222. Academic Press. New Yurk.

175 Enwin. J.A. (1973) in Lipids and Biomembrancis of Lukaryotic Microorganisms (J.A. Erwin. ed.). np. 41-143. Acadcenic Press. New York.

170 Esrahani. M., Kucirka. E.M. Timmons. F.X.. Tragi. S.. Lord. A.E., Jr. and Henry, S.A. (|QX|) J. Supramul. Siruct. (cll. Biochem. 15, 119-128.

177 Fofithini. M., Rudkin. B.B. Cutler. C.J. and Waldron. P.E. (1977) J. Biol. Chem. 252. 3194-3148.

17\% Estabros)k. R.W. (1467) Metheds Fnzyinol. 10. $41-47$.

174 Eybol. C.E. and Simon. R.G. (1970) Lipids 5. 59(1)-59\%.

180 Faas. F.H. and Carter. W.J. (19801) Lipids 15, 453-961.

IXI Faas. F.H. and (arter. W.J. (1983) L.jpids 18, $3,39 \ldots .342$

182 Farias, R.N., Bloj. 8., Morero, R.D.. Sineriz, F. and Trucco, R.E. (1475) Biochim. Biophys. Acts +15, 23!-251.

18.3 Farthing. M.J.G., Keusch, (i.T. and Carey. M.C. (1985) J. Clin. invest. 76, 1727-1732.

IX4 Fee, J.A. Kuila. D., Mather. M.W. and Yoshida. T. (1986) Biochim. Biophys. Acta 853, 153-1 155

it.5 Fen. F.. Canuto. R.A.. Garcea. R.. Avogadro. A.. Villa. M. and Celasci. M. (1971, FEBS Le11. 72. 262-266.

IKt Fernandes, G., Yunis, E.J. and Goxd. R.A. (1976) Proc. Natl. Acad. Sci. USA ?.3. 1279-1283.

187 Filgueiras. M.H. and Op den Kamp. J.A.F. ( 980$)$ Biochim Biophys. Acta 620. 332-337.

IXk Finnerly. W.R. (1978) Adv. Microbial Physiol. 18, 177-233.

189 Fischer. W. (1977) Biochim. Biophys. Acta 487, 84-114.

190 Fleischer, S. and Rouser. G. (1\%65) J. Am. Oil Chemists Six. 42 588-61)7.

191 Fletcher, M.J. and Sanadi, D.R. (|wol) Biochim. Biophy. Acta $51,356-360$ ).

192 Flick, P.K. (1981) Nutr. Rep. Int. 24. 667-674.

19.3 Freake. H.C. and Oppenheimer. J.H. (1987) Prox. Natl. Acad. Sci. USA 84. 3070)-30174.

194 French. S.W., Jhrig. T.J.. Shaw. G.P. Tanaka. T.T. and Norum, M.L. (1971) Res. Commun. Chem. Pathol. Pharmacol. 2. 56758.5 .

195 Frerman. F.E. and White. D.C. (1967) J. Bacteriol. 44, I8t8 1874.

ISt Fiedmann. N., Gellhorn, A. and Benjamin, W. (1\%6) Isr. J. Med. Sci. 2, 677-68?

197 Fry, M. and Green. D.E. (198(1)) Pruc. Natl. Acad. Sci. USA 77. $6391-6395$.

198 Fry, M. and Green. D.E. (1980) Biochein. Biophys. Res. Commun. 93, 1238-1246.

199 Fry. M. and Grien, D.E. (1961) J. Biol. Chem. 256. 1874-1840.

200 Fukushima. K., Martin. C.E., lida, H., Kitajima, Y., Thompson. G.A., Jr. and Nozawa. Y. (1976) Biochim. Biophys. Acta 431. 165-179.

201 Fulco. A.J. (1977) in Polyunsaturated Fatty Acids (Kunau. W.-H. and Holman. R.T., eds.l, pp. 19-36. Am. Oil Chemists' Socicty. Champaign.

202 Galliard, T. (1973) in Form and Funclion of Phospholipids 
(Ansell, F.B., Hawthornc, J.N. and Dawon. R.M.C., eds.), BBA Library $253-2 \times x$.

203 Gellerich F.N., Bolinensack, R. and Kunc. W. (14X,3) Bicxhim. Birophys. incta 722. 3481-341.

204 Gellmorn, 1. and Benjamin. W. (1Yt4) Buxchim. Biuphys. Acta 84. $167-179$.

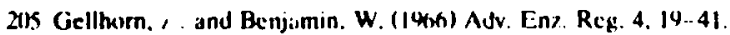

206 Gerschenm 3, L.E.. Harary. I. and M(ad. J.F. (1\%,7) Biochim. Biophys. Ac a 131, 50- $\$$.

217 Gerschensinn L.E., Mead, J.F. IIaran. I. and Haggerty. D.F. Jr. (1467) Buthim. Bikphys. Acta 131, 42-49.

208 Ghadiminejai'. I. and Saggerson, D. (1991) Biochim. Biophys Acta 10K3, 16.-172.

209 Ghosh, A., C7. dralampous, F., Sison, Y. and Borer, R. (14.x1) ]. Biwl. Chem. 23i, 2522-252x.

210 Giacobino. J.P Moinat. M., Mizzin. P., Siegrist-Kaiser, C.A., Seydour. J. and Girardier. L. (1949) Mol. Cell. Endocrinol. 6I. 217-225

211 Gibson. r'A., NoMurcbie, ['.J., Charnock, J.S. and Knecbone. G.M. (1964) Lipi ts 19, 442-951.

212 Giles. R.E.. Blat.: H.. Cann. H.M. and Wallace. D.C. (| $4 \times 11)$ Proc. Natl. Acad. Sci. USA 77, 6715-6779.

213 Gloster, J. and Hi tris, P. (19(ry) (ardiovas. Res. 3, 45-51.

214 Gloster, J. and H: ris, P. (1970) (ardichasc. Res. 4. 1-5.

215 Gnoni, G.V., Land iscina. C.. Ruggiero, F.M. and Ouagliaricllo, E. (1963) Biochim. Biophys. Acla 751. 271-279.

216 Gold, P.H., Gee, M 'i' and Strehter, R.L. (19t(x) \}. Gerontol. 23, $509-512$.

21; Goldfine, H. (1982) ierr. Top. Memhr. Transp 17, 1-43.

218 Gormaghtigh, E., I rasseur. R. and Ruysschacr. J.-M. (.1982) Biochem. Biophys. R.s. Commun. 104. 314-320.

21 y Gordun. E.R.. Richioan. J.. Arai. M. and Licher. C.S. (19K2) Science 216. 1319-131.

220 Grubern, M.. Yazbe.k. J.. Chapey. M.-F.. Diolec, P. and Moreas. F. (I $(90))$ Bio him. Biophys. Acta 1015. 334-340.

221 Gould. J.M. and Crener. W.A. (1977) J. Biol. Chem. 252. $5875-5882$.

222 Grantham. B.D. and 'ammit, V.A. (14xk) Biwchem. J. 249. $404-414$.

223 Grav, H.J. and Blix, A.I (1974) Science 2(14, 87-89.

224 Gray. G.M. and Maciar ine. M.G. $(: \psi / 1)$ Biochem. J. XI. 4X(1)488.

225 Greenberg. A.H., Nejjar. S. and Blizzard. R.M. (1974) in Ilund. honk of Physiology. Se'c 7: Endicrinology. V(ll. 3. I hyroid (Greep. R.O.. Astwex)d. 1 B., Greer. M.A. Solknkin. D.H. and Geiger. S.R., eds.) pp. 37; - 3k9. Am. Physiol. S(x.. Washington.

226 Greenterg. M.L.. Hubbel S. and Lam. C. (198X) Mol. Cell. Biol, 8, 4773-4779.

227 Grinna, L.S. (1977) Mech. 1 eeing Dev. 6. 197-2015

228 Grien. A.R.. Wanders, R.J. 1.. Westerhoff. H.V.. Van der Meer. R. and Tager, J.A. (1982) J. Biol. Chem. 257, 2754-2757.

229 Grogan. W.M. (1961) Lipids 16, 9401-442.

230 Groner, G.A. and Heffron J.J.A. (1979) Anesth. Analg. SK. $76-81$.

231 Gross, M.D., Whitty. A.J. an' Foà. P.P. (197k) Cancer Res. 32. $1978-1982$.

232 Gross, N.J., Getz, G.S. and R: Sinowitz, M. (|469) J. Biol Chem. 244, 1552-1562.

233 Gruner. S.M. (1985) Proc. Natl Acad. Sci. USA 82, 3665-3669.

234 Gudbjamason, S., Doell, B. a:d Oskarsdóttir. G. (197k) Acta Biol. Med. Germ. 37. 777-784.

235 Gudbjarnason, S. and Oskando:tir. G. (1975) Rec. Adv. Card. Struct. Mctab. 6, 193-203.

236 Gudbjarnason, S. and Oskarsodót ir. G. (1977) Biochim. Biophys. Acta 487, 10-15.

237 Guerrieri. F. Zanotti. F.. Caprzzi. G.. Colaianni. G., Ronchi, S and Papa. S. (1991) Bixchim. Biop'yss. Acta 1059, 348-354.
2.8 Ciutknecht, J. (1984) J. Membr. Biol. 82, 19.5-112

239 Gutknecht. J. (1987) J. Bioenerg. Bumemhr. 19. 427-442.

241 Gitknecht. J. (1987) Proc. Natl. Acad. Sci. USA \$4. 6443-6446.

241 Gutknecht. J. (1984) J. Memhr. Biol. l(K). 8.3--43.

242 Hack. M.H. and I lelmy. F.M. (|Q4|) J. Planar Chromatogr. 4. (x) $(1)-x 4$.

24.3 Hackenhrock. C.R. (1'k8) Prix. Natl. Acad. Sci. USA 01. 59\%olls.

244 Hacst. C.W.M.. De Gier, J. and Van Deenen. L.L.M. (1949) Chem. Phys. Lipids 3, 413-417.

245 Hafner, R.P. (1987) FEBS Lett. 224. 251-256.

24h Hafner, R.P. and Brand. M.D. (1964) Biochem. J. 250, 477-484.

247 Hafner. R.P., Brown. G.C. and Brand. M.D. (1990) Biochem. J. 265, $731-734$.

24k Hafner. R.P., Leake, M.J. and Brand, M.D. (1989) FEBS Lett. 248. 175-178.

244 Hafner. R.P.. Nobes. C.D. McGown. A.D. and Brand. M.D. (19xk) Eur. J. Biochem. 178. S11-518.

250) Haggerty. D.F.. Jr., Gerschenwm. L.E., Harany, I. and Mead, J.F. $(\mid \% 7)$ Biochem. Bisphys. Res. Commun. 6. 558-574.

251 Haines. T.H. (198.3) Proc. Nall. Acad. Sci. USA 80). 160)- 164.

252 Hall, J.C. Sordahl, L.A. and. Siefko, P.L. (IW(x)) J. Biol. Chem. 235. $1534-1534$

253 Hall. P.F.. Watanuki, M., DeGroxt, J. and Rouser. G. (1979) Linids 14. 14k-151.

254 Hallgren. B., Lundyuist, C. G. and Svantorg. A. (1966) Acta Med. Siand. 179. 447-452.

25.5 Hamman. 11.C. and Haynes. R.C.. Jr. (1983) Arch. Biochem. Biophys. 223. 85-94.

256 Hansford. R.G. (1978) Biochem. J. 170. 265-295.

257 Hansford. R.G. (1980)) in The Aging Heart (Aging. Vol. 12) (Weisfeldt. M.L.. ed.). pp. 25-76. Raven Press. New York.

25x Hansford. R.G. (1983) Bixchim. Bioph, s. Acta 726, 41-80.

259 Haran), Y.. DePalma, R.G.. Lavine. L. and Miller, M. (1972) Dialetes 21, 257-27I.

26xl Harary, I., Gerschenson. L.E., Haggerty, D.F., Jr., Desmond, W. and Mead. J.F. (1\%(7) in Lipid Metaholism in Tissue Culture Cells (Rothhlat, G.H. and Kritchersky, D., eds.). pp. 17-31. Wistar Insi. Press. Philadelphia.

2 (h) Harh. J.S. Comte. J. and Gautheron. D.C. (1961) Arch. Bioxhem. Biophys. 20k. 3K5-31X.

2h2 Haslam. J.M.. Proudlixk. J.W. and Linnane, A.W. (1971) Bioencigetici 2. 351-370.

2n.3 Haslarii. J.A... Spith:!!. T.W Linnane A.W. and Chappell, J.B. (1973) Biochem. J. 134, 949-957.

264 Hawithorne. J.N. (1982) in New Comprehensive Biochemistry Vol. 4. Phospholipids (t fawthorne, J.N. and Ansell. G.B., eds.). pp. 263-278. Elsevier. Amsterdam.

2h5 Hayashida. T. and Portman, O.W. (1\%,3) J. Nutr. 81, 103-109.

26n Hdzel, J.R. (1973) in Effects of Temperature on Ectothermic Organisms. Ecokgical Implicutions and Mechanisms of Compensation (Wieser, W.. ed.), pp. 55-67. Springer-Verlag. New York.

267 Hazel. J.R. and Prosser. C.L. (1970) Z. Vergleich. Physiol. 67. $217-228$.

2kx Hazel. J.R. and Prosser. C.L. (1974) Physiol. Rev. 54, 620-677.

¿'9 Heggtveit, H.A.. Herman. L and Mishra, R.K. (1964) Am. J. Pathol. 45. 757-782.

270 Hegner, D. (1980) Mech. Ageing. Dev. 14, $101-118$.

271 Hellingwerf, K.J., Lolkema, I.S., Otto. R., Neijssel, O.M., Stuuthamer. A.H., Harder, W., Van Dam, K. and Westerthoff. H.V. (1982) FEMS Microoiol. I elf. 15. 7-17.

272 Herring. F.G., Krisman, A., Sedgwick, E.G. and Brags. P.D. (1965) Biochim. Bioptys. Acta 819, 231-240.

273 Himms-Hagen. J. (1976) Annu. Rev. Physiol. 38, 315-351.

274 Himms-Hagen. J. (1984) N. Engl. J. Med. 311. 1549-1558.

275 Himms-Hagen. J. (1990) FASEB J. 4. 289)-2848. 
27h Himms-Hagen. J., Behrens. W.. Milirhead. M. and Hhous. A (1975) Mol. Cell. Biochem. 6, 15-31.

277 Hoch. F.L. (1\%22) N. Engl. J. Mcd. 266. 446-\$54. 448-5015.

278 Hoch. F.L. (1962) Physiol. Rev. 42. 6015-671.

279 Hoch. F.L. (14\%8) Arch. Biochem. Biophys. 124. 23K- 247.

280 Hoch. F.I. (1971) in Physiological Chemistry (Masoro, E., cd.). Vol. 3. 21 ? pp.. Saunders. Philadelphia.

281 Hoch. F.L. (1977) Arch Biochem. Biophys. 17k, 535-54.5.

282 Hoch. F.L. (1982) in Phylogenic Aspects of Thyroid Hormone A.ctions. 19th Gunma Symposium on Endocrinology (Institute of Endocrinologs: Gunma University, eds.). pp. 139-149. (enter for Academic Publications Japan. Tokyn.

283 Hoch, F.L. (1982) J. Mol. Cell. Cardiol. 14. 81-86.

284 Hoch. F.L. (19k8) Prog. Lipid Res. 27. 199-27!?

285 Hoch. F.L. (1988) in The Roots of Modern Biochemistry. Fritz Lipmann's Squiggle and its Consequences (KJeinkauf, H., Von Döhren, H. and Jaenicke, L., eds.), pp. 645-655, De Gruyter, Berlin.

286 Hoch. F.L. and Chavis. T.R. (1982) Fed. Proc. 41. 968.

287 Hoch. F.L. and Chavis. T.R. (198.3) Fed. Proc. 42. 325.

288 Hoch. F.L.. DePierre. J.W. and Ernster. L. (19*(1)) Eur. J. Bixchem. 109, 301-30\%.

$2 \times 9$ Huxh, F.L. and Höijer. B. (|YK1) J. Bixenerg. Biomembr. 13. 25-35.

290 Hoch. F.L. and Lipmann, F. (1954) Proc. Natl. Acad. Sci. USA 40, $909-921$.

291 Hoch, F.L.. Subramanian. C.. Dhopeshwarkar. G.A. and Mead. J.F. (1981) Lipids 16. 328-335.

292 Hoek. J.B. and Taraschi, T.F. (1988) Trends Biochem. Sci. 13. $269-274$

293 Holman. R.T., Johnson. S.E.. Gerrard. J.M., Maucr. S.M.. Kupcho-Sandberg. S. and Brown. D.M. (1983) Proc. Natl. Acad. Sci. USA 80, 2375-2379.

244 Holman. R.T., Johnson. S.B. isld Hatch. T.F. (1982) Am. J. Clin. Nutr. 35, 617-623.

295 Hopfer, U., Lehninger, A.L. and Lennar, W.J. (1970) J. Membrane Biol. 2. 41-58.

29) Holtzman. D. and Morere, C.L. (1975) J. iteuruchem. 24. l.17 ; in15.

297 !. orruvin. D.F. (1981) Med. Hypritheses 7. 1211-1220.

298 Hurrum. M.A. and Tobin. R.B. (19\$8) FASEB 1. 2, A775.

299 Hurrum. M.A. and Tobin. R.B. (IY40)) FASEB S. 4. A.303.

300 Horrum. M.A.. Tobin. R.B. ind Ecklund. R.E (1\%(i)) Mol. Cell. T:ndocrinol. 6X, 137-111.

301 Horton, A.A. and Spencer, J.A. (1981) FEBS Lett. 133, 134- 141.

302 Horton. A.A. and Spencer. J.A. (i981) Mech Ageing Dev. 17. 253- 259 .

303 Hor:áth, L.1.. Drees, M., Beyer, K., Klingenterg. M. and Marsh, D. (1990) Biochemistry 29. I1K604-10669

304 Hosein E.A., Hofmann. 1. and Linder. E. (1977) Arch. Biochem. Biophys. 183. 64-72.

15 Hostetler. K.Y. (1982) in New Coniprehensive Biuchemistry Vol. 4. Phospholipids (Hawthornc. J.N. aid Anselt. G.B.. eds.). pp. 215-261. Elsevier. Amst:- J.m.

3h Houle, A.. Tëcly. F.. Aghion. J. and Lethlanc. R.M. (19;:2) J. Lipid Re:. 23. $490-502$.

307 Hovius, R., Lambrechts. H., Nicolay. K. and De Kruijf, B. (1490) Biochim. Biophys. Acta 1021, 217-226.

308 Hsu, C.M.L. and Kummerow, F.A. (1577) Lipids 12, 486-494.

309 Hsung. J.C. and Haug, A. (1977) FEBS Lett. 73, 47-50.

310 Huang. Y.S., Horrobin, D.F., Manku, M.S., Mitchell, J. and Ryan, M.A. (1984) Lipids 19. 367-370

311 Hubbard. D.D., McCaman. R.E.. Smith, M.R. and Gibson, D.M. (!\%1) Biochem. Biophys. Res. Commun. 5. 334-343.

312 Huber. R., Kurr, M., Jatonarich. H.W. and Stetter, K.O. (1ysy) Nature 342, 8.33-8.34.
31.3 l!übner, W., M.ntsch, H.M. and Katts, M. (|w)|) Bituchint Bicophys. Acla lokers. 16x-174.

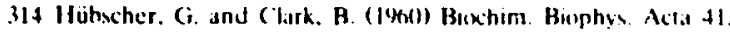
$45-54$.

315 Hunter, K. and Rence, A.11. (1972) Bixchim. Bionhys. Actal 26). 6.39) -6.53

31h Ikemol(o, H., Hironhige. T. and ltoh. S $(\mid 467)$ Jpn. J. Physiol. 17. $516-522$.

317 Imai. Y. (|का|) J. Biushem. Tukyo 49. 612-644.

318 Innis. S.M. and (lundinin. Af T. (1981) Bixilsem. J. 14.3. 155-167.

319 Innis. S.M. and Clandinin, M.T. (1981) Biochem. J. 148. 231-234.

321) Joannou. P.V. and Golding. B.T. (1979) Prog. Lipid Res. 17. 279-318.

321 Ishinaga, M.. Sato, J.. Kitagawa, Y.. Sugimoto, E. and Kito, M. (1982) J. Biochem. Tokyo 92, 253-263.

322 islacl, Y.. Khanna. J.M., Orrego, H., Raci.amin. G.. Wahid. S. Britton. R.. Macdonald. A. and Kalant. H. (1979) Torug Akcohol Depend. 4. $1(19)-118$

323 Israel. Y.. Videla. L. and Bernstein. J. (1975); Fed. Prox. 34. 2052-2059.

324 Jakmoir, S. Gelz. G.S.. Rabinowitz. M., Jakoh. H. and Switt. H. (1471) J. Cell Biol. 4K, 49(1)-5(1).

325 Jakowcic, S., Haddock, J., Getz. G.S., Rabinowitz, M. and Swift, H. (1971) Biochem. J. 121. 341-347

326 Jakıcic. S., Swift. H.H., Gross. N.J. and Rahinuswitz. M. (1978) 1. Cell Biol. 77, $x \times 7-910 i$.

327 Jasull, E.L., Mulier, P.J., Meyer. E.A. and Mors, S.A. (|48|) Mol. Biochem. i artivtol. 2. 187-196.

328 Jennings. R.B. and Ganote. C.E. (1976) Circ. Res. Suppl. 3x. I.-X(i-y).

324 John. P. and Hamilton. W.A. (1971) Eur. J. Bikchem. 23, 52X532.

330 John, P. and Hamilton, W.A. (1975) FEBS Lett. 10. 246-24k.

33) John. P. and Whatley, F.R. (1975) Nature 254. 495-498.

332 Johnson. J.D. and Cornatzer, W.E. (I 94$)$ Prox. Sic. Exp. Biol. Med. 131, 474-478.

333 !nhorion JH Cirula, E.A.. Staerkel, R.. Fung. B.M. and Mangum, B.L. (1980)) J. Membr. Biol. 56, 44-53.

334 Johnwon, P.C.. Posey, A.F., Patrick. D.R. and (aputto, R. (1958) Am. J. Physiol. 192, 279-282.

335 Johnswn. R.M. and I10. T. (1\%65) J. Lipid Res. h. 75-74.

336 Jollow, D.. Kellerman. G.M. and Linnanc. A.W. $(\mid \%(x)$ J. (ell Biol. 37, 221-230.

337 Jonah. M. and Enwin. J.A. !!'?:? 2:intin. Biophys. Acta 231. $\times 0.42$.

338 Jones, C.W., Brice, J.M. Downs, A.J. and Drozd, J W. (1975) Eur. J. Hiuchem. 52, 265-271.

339 Jump. D.B. and Oppenheimer, J.H. (1985) Endecrinology 117. 2259-2266.

340 Kabnick. K.S. and Peattic. D.A (1991) Am. Sci. 79, 34-43

;41 Kadenbach, B. (1466) Biochem. Z. 344, 49-75.

342 Kagawa, Y. and Ariga, T. (1977) J. Biochem. Tokyo XI. ||6|1165 .

34.3 Kagawa, Y. and Sone, N. (1974) Method: Enzymol. 55. 364-372.

34t Kaneda. Y. and Goutsu. T. (19\$א) Ann. Trop. Med. Parasitol. 82. K3-90.

345 Kaneko, H. Hosohar:t. M. Tanzka. M. and lloh. T. (1976) Lipids 11, 837-844.

346 Kanemasa. Y.. Akamalsu, Y. and Nojima, S. (1477) Bicchim. Biophys. Acta 144. 382-341.

347 Kanungo. M.S. and Prosser. C.L. (1954) 1. Cell. Conp. Physol. 54. 265-274.

348 Kaplan, R.S., Oliveira, D.L. and Wilson. G.L. (IQXX]) Arch. Bixchem. Biophys. 281, 181-194.

349 Kargapolov. A.V. (1979) Brokhimiya 44, 293-24h. 
350 Kates, M. and Baxter, R.M. (19nzi Can. J. Buxchem. Physiol 4l. 1213-1227.

351 Kater, M and Paradis. M. (1973) (an. J. Bickehem. 5i. 184-147.

3.52 Keith. A.D. Wonnewexi, B.J. Henn. S. and Villiams..$C$.

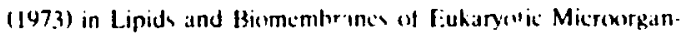
isms (E:rwin, J.A. ed.). pp. 259-321. Aciddenti: Pecess. Niu York.

35: Keogh. J.M. Matthens. P.M.. Seymour. A.-M. and Radda. (j.K. (1985) Ad. Myocardicol. h. 244-314.

354 Keräinen. A. Kankare. P. and Hallman. M. (1982) 1.ipids 17. $155-159$

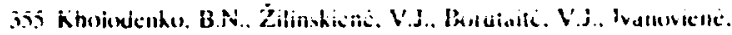
L.J.. Toleikis. A.J. and Prañkevičus. A.K. (19k7) HEBS L.ett 223, 247-250).

35h Kholodenko, B.N., Żilinskiené, V.J., Bururaite. V.J., Ivanowiene. L.J.. Tolcikis. A.J. and Praškevičius, A.K. (I9X8) Biohhimiya 53. $10(x)-1012$

357 Kielicy. R.K. (1952) Cancer Res. 12. 124-128.

$358 \mathrm{Kim}$, J.H. Shrago, E. and Elwon. C.E. (1988) Mech. Ageing Dev. 46. 279-291.

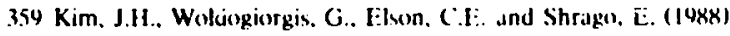
Mech. Apcing Dev. 4h. 2h3-277.

36) Kito, M.. Aihara, S. Kall, M. and Hata. T. (1472) Biechim Biophys. Actis 26). 475-478.

361 Kleibe: M. (|צt/) The Fire of life. An Intseduction to Animal Entrgi' :es. 4.54 pp. John Wiley and Sons. New York.

362 Klein. A.ll, Jenkins, J.J., Keviczky. A. and Fisher. D A ! lus!! Am. J. P iysiol. 241, E449-E453.

36.3 Klingenherg. M. (1904)) Tyends Biochem. Sci 15. I(18-11?.

364 Klingenterg. M. and Winkler. E. (1965) l:M(B) J. 4. 3:387-.3(k)2.

36.5 Knoll, $G$ and Brdiczka. D. (19K.3) Biochim. Biophys. Acla 7.3 .3$. 1112-111).

36t Kolodzicy. M.P. and Zammit V.A. (144(1) Biochem. J. 272. $421-425$.

367 Kramer. I K.G. (1980) Lipids 15, 651-66t).

368 Kramer. J.K.G., Farnworth, E.R. and Thompson. B.K. (1985) Lipids 20.6.35-644.

369 Kramer, J.K.G. and ITulan, H.W. (1977) Lipids 12, $159-164$.

370 Krämer, R. and Klingenterg. M. (19801) FASE:B J. 119. 257-260)

371 Krishnamocorthy. G. and Hinkle. F.C. (194d) Bicchemistry 23. 1641)-16.45.

372 Krulwich. T.A. and Gutfanti. A.A. (1966) Methods Enzymol. 125. 352-36.5.

373 Kuksis. A. (1978) in Handhuxk of Linid Research Vol. I. Falty Acids and Glycerides (Hanahan. D.J.. ed.). pp. 3KI-442. Plenum Press. New York.

374 Kunz. W. Bohnensiack. R.. Bühme. C.. Küster. U., Letko. G. and Schïnfeld. P. (1981) Arch. Biochem. Biophys. 2(y. 214-221).

375 Kunz. W., Gellerich. F.N.. Schild. L. and Sibïnftld. P. (148x) FEBS 1ett. 233, 17-21.

376 Lacumbe. C. and Lutuchinsky. B. (19kx) Biwhim. Biophys. Actit \%)1, 183-187.

377 Lii, A., Casu, M.. Meloni, C. and Muxcatello. U. (1989) Bicxhem. Biophys. Res. Commun. 161. 979-986.

378 Landriscina, C.. Megli. F.M. and Quagliariello, E. (1976) Lipids II. $61-66$.

379 Langwiorthy. T.A. (1982) Curr. Tup. Metubr. Trafisp. 17, 45-77.

38) Langworthy. T.A. Mayberry. W.R. and Smith. P.F. (1976) Biochim. Biophys. Acta 431, 550)-569.

381 Lardy, H. and Wellman. H. (1952) J. Biol. Chem. 195. 215-224.

382 Ledwoch. W.. Greeff. K. Heinen. E. and Nosck. E. (I974) J. Mol. Cell. Cardiol. 11, 77-89.

383 Lee. N.H. and Shapiro. I.M. (1974) Calcif. Tissue Res. 16. $277-282$.

384 Lee. N.11. and Shapiro. I.M. (1974) Anal. Biochem. 58. 117-122.

385 Legname. A.H. Salomón de Legname. H.. Sánchez. S.S..
Sinchez Riera, A.N. and Fernandez. S.N. (1972) Der. Biol. 29. 2i:? $2 \because 2$.

38's Lehninger A.1. (196t) The Mifochondrion. Molecular Basis of Structure and Function, 263 pp. W.A. Benjamin. Neu York.

367 Lenting. H.BM. Neys. F.W. and Van den Bexch, H. (1988) Bischim. Bionhys. Acta W61. 129-1.38.

$38 x$ l.crn:t, l:.. Shug. A.L. Elsin. ( and Shragn, E.. (1972) J. Biol. (hem. 247. 1513-1514.

iso l.ctls, V.. Shaw. P.. Shapiro, L. and Henry, S. (1982) J. Bacteriol. 15!. 1269-127x.

391 Lewy. $M$ and Joncourt. M. (1973) (.R. Six. Biol. 167, 848-851.

391 lewir. M.B. and Timiras. P.S. (1984) Mech. ARe.ng Dev. 24. $343-351$

392 Li. P.M. Morgan. J.L.. Nilsmon. T.. Ma. M. and Chan. S.I. (198X) Biochemisty 27. 7538-7546.

39.3 Lin. (.-1 . and Klingenherg. M. (1982) Biochemistry 21, 2950$24.5 \mathrm{~h}$.

394 Liškovia, Z.. Strunecká. A. and Drahola. Z. (1974) Prysiologia Bohemeslovaca 23. 221-22x.

395 Lipez-Aloratalla. N.. Scgovia, J.L. and Santiago. E. (197.2) Rev. F..... .isiol. 24. 329-334.

3 W lowe. A.G. and Jones. M.N. (1984) Trends Bischem. Sci. 9. $11-12$.

397 Lundquist. C.C. and Svanturg. A. (14t4) Acta Med. Scand. 17.6. $7111-704$.

34k Lyman. C.P., OBBrien. K.C.. Greene. G.C. and Papafranges.

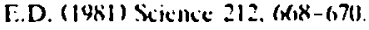

344 Lyoms. J.M. (19?2) ('nyobiology 9. 341-350.

tix) Macfarlane. M.(i. (1961) Bickhem. J. 78, 4450.

HII Maddaiah. VT (iqu(x)) FASEB J. 4. 151.3-151X.

H12 Maddaiah. V.T ind (íijin. S. (1986) Endocrinulogy 119. 250252.

4113 Mahfouc. M.M. and Kummereru. F.A. (1989) Lipids 24. 72;-732.

4)t Mahfouz. M.M. Simith. T.L. and Kum erow. F.A. (1984) Bischim. Bioplays. incid i(KK. 70)-74.

4)S Mahfouz. M.M. Smith. T.1. and Kummer(ww. F.A. (1989) Biochim. Biopliys. Acta lixM. 'S-83.

Hik Mak. 1.T., Shrago, E. and Elson. C.E. (1983) Biochim. Biophys. Acta 722, , (12-3(x).

417 Malmström. B.G. and Nilssin. 1. (IYKX - Ann. PI.Y. Acad. Sci. 550. 17?-187.

4116 Makoney. P.C. (1979) J. Bacteriol. 141). 197-205.

H(Wy Maloney. P.S. (1987) in Excherichia coli and Salmonella $n$ phimurium. Cellular and Molecular Biology (Neidhardt. F.C.. cat.). Vol. 1. pp. 222-243. Am. Sic. Microhiol.. Washinglon.

f10 Mannnall. D. and Getz. C.S. (1973) in Lipids and Birmembrance of Eukatyotic Micronrganisms (Enwin. J.A. ed.). pp. 145-145. Academic Picss. New York.

11) Manocha. M.S.. San-Blas. G. and Centeno. S. (19X0)) J. Gen. Microbiol. 117, 147-154.

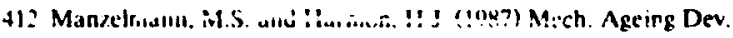
39. $2 \times 1-2 \times 8$.

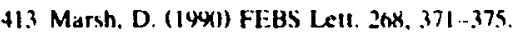

4lt Marrius, C. and He'ss. B. (1451) Arch. Biochem. Biophys. 33, $4 \times 6-4 \times 7$.

\$15 Masini. A. (eccarelli Stinzani. D. and Muscatello, U. (1984) Bischim. Biophys. Acla 7(17. 1.31-137.

416 Masoro. F J.. (implon. C.. Yu. B.P. and Bertrand. H.A. (1983) Fed. Prox. 42. 13).

417 Massro, E.J. Riwell, L.B., Midunald. R.M. and Steiert. B. (16*6) J. Biol. Chem. 241. 2626-26.34.

418 Masitti. L... Lenaz. G.. Spisni. A. and Urry. D.W. (1974) Biochem. Biophys. Res. Commun. 56. 892-897.

419 Matsubara. T. and Tochino. Y. (1969) J. Bi(x'lem. Tokyo o6, $397-4104$ 
420) Matsubara, T. and Tochino, Y. (1970) J. Buchem. Tokyo hx, $731-736$.

421 Mazat, J.-P., Jean-Bart. E., Rigoulet, M. and Guèrin. B. (I9kx) Biochim. Biophys. Acta 449. 7-15.

422 McCarty. R.E.. Douce, R. and Benson. A.A. (197.3) Bischim. Biophys. Acta 316. 26ti-270.

423 McCay, C.M. Cr.wwell. M.F. and Maynard. L.A. (1939, ¿. Nutr. 10. 63-79.

424 McElhaney, R.N. (1982) Curr. Top. Membr. Transp. 17, 317-361l.

425 McKay. A., Quilter, J. and Jones. (.W. (1962) Arch. Microbiol. 1.31, 43-50.

426 McLaughlin, J. and Mitrowitch, E. (1975) Comp. Biochem. Physiol. 52B, 487-497.

427 Mead. J.F., Alfin-Slater, R.B., Howston, D.R. and Popják. G. (1986) Lipids. Chemistry. Biochemistry. and Nutrition. p. 145, Plenum Press, New York.

428 Mercuri, O.. Peluffo. R.O. and Brenner. R.R. (19(6) Biochim. Biophys. Acta 116, $409-411$.

429 Meyer, D.J. and Jones, C.W. (1975) Eur. J. Biochem. 36. 144157.

430) Miceli, J.N. and Ferrell. V.J. (1973) Lipids $x .722-727$.

431 Miller. B.C., Lau, H.W.. Tyler, N.E. and Cuttam. G.L. (1988) Biochim. Biophys. Acta \%2, 25-36.

4.32 Miller. N.G.A.. Hill, M.W. and Smith. M.W. (1976) Biochim. Biophys. Acta 455, 644-654.

433 Mitchell. P. (1966) Chemiosmotic Coupling in Oxidative and Photosynthetic Phosphorylation. 192 pp.. Giynn Reseastch, Bodmin.

434 Mitchell. P. (1967) Fed. Proc. 26. 1370-1379.

435 Mitchell, P. (1976) Biochem. Soc. Trans. 4. 399-430.

436 Mitchell, P. (1988) Ann. iN.Y. Aciud. Sc: 550. 185-198.

437 Mitchell. P. and Moyle. J. (1967) Bioche n. J. 104. 586-61)0. Eqn. 25.

438 Mitchell, P. and Moyle. J (1988) Eur. J. Biochem. 7, 47!-484.

439 Miltnacht, S., Jr. and Farber. J.L. (1981) J. Biol. Chem. 2S6, $3199-.320 \%$.

440 Mittnacht. S., Jr.. Sherm in, S.C. and Farber. J.L. (1979) J. Biol. Chem. 254. 987i-9878.

441 Miyahara. M.. Kitazoe. Y.. Hiraoka. N.. Takeda, K.. Watanabe, S.. Sasaki. J., Okimasu. E.. Osaki. Y.. Yamamoto. H. and Utsumi, K. (19\%4) Biol. Neonate 45. 129-141.

442 Moore. A.L., Dry, I.B. and Wiskich. J.T. (199i) Plant Physiel. 95. $34-40$

443 Moore, P.K. and Hotil, J.R.S. (1980) Nature 248. 271-273.

$\$ 44$ Morton. R., Cunningham. C.. Jester, R.. Waite. M., Miller. N. and Morris, H.P. (1976) Cancer Res. 36, 3246-3254

445 Morton. R.E., Hartz. J.W., Reitz. R.C., Waite. B.M. and Morris. H.P. (1979) Biochim. Biophys. Acta 573, 321-331.

446 Mory, G.. Ricquier. D., Pesqutés, P. and Hémun, P. (1981) J. Endocrinol. 91, 515-\$?4.

447 Müller. M.. Chenevid. D. and Carafoli. E. (1984) Eur. J Biochem. 140, 447-242.

4.8 Mi:Her. M.. Moser, :... Cheneval. D. and Carafoli. E. (1985) J Biol. Chem. 260, 3k30-38.4?

449 Murfit:, R.R. and Sanad:, D.R. (1978) Mech. Ageing Dev. 8. 197-201

450 Murphy, M.P. (1989) Biochim. Biophys. Acta 977. 123-141.

451 Muscari. C., Frascaro, M., Guanieri, C. and Caluarera. C.M. (19\%0) Biochim. Biophys. Acta 1015, 2(00-204.

452 Nagle. J.F. (1987) J. Bioenerg. Biomembr. 19, 413- 426.

453 Nakamura. M., Nakatini, M., Koike, M., Torii, S. and Hiramatsu. M. (1961) Proc. Soc. Exp. Biol. Merd. i08, 315-319.

454 Nalecz. R.A., Bolli. R., Wojtczak, L. and Azzi, A. (1986) Biochim. Biophys. Acta 851, 29-37.

455 Neidharol. F.C. (1987) in Escherichia coli and Salmonello ty. phimurium. Cellular and Molecular Biology (Neidhardt, F.C.. ed.). Vol. 1. pp. 3-6. Am. Soc. Microbiol., Washington.
4.56 Nerissel. O.M. and Tempest. D.W. (1976) Arch. Microhisl. 110 $305-31 i$

457 Nelsin. (i.J. (1962) 1. Lipid Res. 3. 256-262

4.5 Neni. A.M.. Peluffo. R.O. and Brenner. R.R. (19s()) Lif.ide Is $26.3-268$.

154 Nichulls, D.6 (1974) Fur. J. Biochem. 50. 315. 315

4" Nichoils. D.G. (1976) FFBS Let1. 61, 103-110.

4h1 P.t. IIs. D.G. (197/; Eur. J. Biochem. 77, 344-356

462 Nicholls. D.G. (1982) Bionenergetics. An Introduction to the Cherniosmotic Theory. 190 pp. Arademic Press, New York.

th.3 Nicholls. D.G. (1483) Bioswi. Rep. 3. 4.31-441.

464 Nicholls. D.G. (1984) in New Comprehensive Bickhemistry Vol. 4. Binenergetics (Ernster, 1... ed.), pp. 29-46. Elsevier, Amster. dam.

465 Nicholls. D.G. and Locke, R.M. (1984) Physiol. Rev. 64, 1-64.

tho Nicholls. P. and Wenner, C.E. (1972) Arch. Biochem. Biophys. 151. 206-215.

46- Nichols. B.W. (1965) Biochim. Biophys. Acta 106, 274-279.

46.8 Nichols. J.W. and Deamer. D.W. (1980) Proc. Natl. Acad. Sci. USA 77. 2038-2042.

69 Nilsson. T., Gelles, J., Li. P.M. and Chan, S.I. (1988) Bicchem istry 27, 296-301.

47) Noël, H. and Pande, S.V. (1986) Eur. J. Biochem. 155, 99-102

4 - 1 Nohl. H. (1979) Z. Gerontol. 12, 9-18.

472 Nohl. H., Brecininger. V. and Hegner, D. (1978) Eur. J. Biochem. 9n. $385-3 \times 0$.

47: Nohl, H. and hisnn:r, D. (1978) Eyr. J. Biochem. 82, 563-567

474 Nin̆ii, H. and Krämer, R. (1980) Mech. Ageiıg Dev. 14, 137-144.

475 Norris, F.A. and Powell. G.L. (1990) Biochi ?. Biophys. Acta 11)30, 165-171.

i76 Okano. G.. Matsuzaka. H. and Shimojo. T (198(1) Bıxchim. Biophys. Acta 619. :67-175.

477 Okayasu. T. Curtis, M.T. and Farber. J.L. (1985) Arch. Biochem. Biophys. 236. 638-645.

478 Okuyaina, H., Kankura, T. anu Nojima, S. (1\%67) J. Bixchem. Tokyo 61, 732-737.

479) Okuyama, H. and Nojima, S. (1965) J. Biochem. Tokyo 57, 529-538

4(X) Okuyama, H. Yamada. K., Kameyama, Y., Ikezawa, H., Akamatsu, Y. and N(1)uma, S. (isiri) Bienikeiristry 16, 2668-2673.

481 Om, Y. and White, D.C. (1971) J. Bacteriol. 108, 1065-1071.

482 Oppenheimer, J.H. and Samuels, H.H. (1983) Molecular Basis of Thyroid Hormone Action 498 pp.. Academic Press, New York.

483 Orrego. H. Blake. J.E.. Blendis. L.M., Compton. K.V. and Israel. Y. (1987) N. Engl. J. Med. 317. 1421-1427.

484 Oshima. M. and Miyagawa. A. (1974) Lipids 9, 476-480.

485 Oslage, H.J., Petersen, U. and Seher. A. (1983) Fette Seifeii Anstrichmittel 85, 177-184.

$\$ 86$ Ou, W.-J., Ito, A., Umeda, M., Incue, K. and Omura. T. (1988) J. Biochem. Tokyo 103, 589-595.

487 Uwen. C.S. and Wilson. D.F. (1974) Arch. Biochem. Biophys. 161, 581-591.

488 Padan. E. and Rottenterg. H. (1973) Eur. S. Biochem. 40, 431-437.

4xy Padan, E. and Srhildiner. S. (1986) Melhods Enzymol. 125, $337-352$

4 (X) Padowan. A.C.. Dry. I.B. and Wiskich. J.T. (1989) Plant Physiol. 90. 928-933.

491 Palmer. J.W., Schmid, P.C., Pfeiffer, D.R. and Schmid, H.H.O. (1981) Arch. Biochem. Biophys. 211. 674-682.

492 Paltauf, F. and Johnston. J.M. (1970) Biochim. Biophys. Acta 218. 424-43n.

493 Paltauf, F. and Schatz. G. (1969) Biochemistry 8. 335-334.

494 Paradies, G. and Ruggiern, F.M. (1988) Biochim. Biophys. Acta 035. $:-86$. 


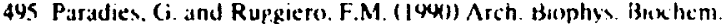
27k, 425-4301.

44) Paradies. (; and Ruggicro. F.M. (I\$x|) Binkhim Biopw. Acta 1016. 207-212.

497 Parades, (i. and Ruggero. F.M. (Iwn) Bushim. Bion wys Acta $1014.133-13 \mathrm{~h}$

496 Parkes. J.(i. and Thompun. W (1970) Biochim. Biophys. Acla 14\%. Ih2-164.

494 Parks. R.E.. Jr., Adler. J. and (openhaver, J.11., Jr. 11455) J. 3iol. Chem. 214. 643-698

S(k) Parwons, D.F., Williams. (i.K.. Thomnoon. W.. Wilson. D). and Chance, B. 11467) in Mitcchondrial Siructure and (omparemen. tation Couagliatricllo, E... Papa. S.. Slater. E.C. and Tagtr. J.M.. eds.). pp. 29-70, Adriatica Editrice, Bari.

501 Parvin. R. and Pan'se. S.V. (1979) J. Biol. Chcm. 254. 542: -5424.

502 Pallon. G.M.. Fanuio. J.M. and Ribuns. S.J. (1982) J. Lipid Res. 23. $190-1 \%$.

S0.3 Pearce. P.H. and Kakulas. B.A. (1980)) Austral. ' Exp. Bial. Med. Sci. 58, 397-41k:

504 Pedersen. P.L., Greenawalt, J.W.. Chan. T.L. and Marris, H.P. (1470) (ancer Res, 30) 26201-2626.

51IS Pedersen. P.L. and Morris, H.P. (1974) J. Biol. Chem 24'3. $3327-333.34$

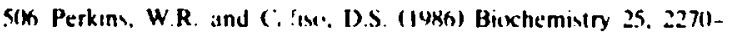
$227 b$.

5(17 Perkins, W.R. and (afix), D.S. (14X7) J. Bickenerg. Bismemhr. 19, 443-455

Sur Pelrogailo. V.A. Cicco. T. and Gnoni. (j.V. (1945) Bull. Six. Ital. Biol. 61. 66.3-to64.

5(Y) Platnet. W.S. Palnayak. H.C. and (halfec. R.R.J. (1472) Proc

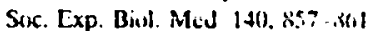

510 Pollak. J.K. (1975) Biochem J. i. 11 . 477 . 4kx

SII Pool. R.P. (|yx/) Science 247, ISx- | $x \mid$.

512 Powell. G.L.. Knowles. P.F. and Marsh. D. (1485) Bicxhim. Biophys. Acta 816, 191-194.

S13 Powell, G.L.. Knowles, P.F. and Mginh. D) (14א7) Buchemistn; 26. $8138-8145$.

514 Powell. G.L., Knowles, P.F. and Marsh. D) (14x)) Bickhemistn 29. $5127-5132$.

515 Powell. G.L. and Marsh. D. (1985) Bic chemisiry 24, 29x)2-24(x)

516 Prats, M.. Teissić, J. and Tocanne. J.-F. (14k6) Nature 322. 756-758.

517 Prats, M., T(xanne. J.F. and Tessic: J. : 1987) Fur. J. Bickem 162. 379-385.

518 Prusiner. S.B. Cannon. B.. (hing. T.M. ar.d Lindherg. (). (1फK) Eur. J. Bixchem. 7. 51-57.

519 Prusinet. S. B. and P(K. M. (14t(k) Nature 2211. 235-237.

950 Pye $V(1473)$ in Effects of Tem alure on Ectolicernic Organ isms. Ecological Implications and Mechanisn 's of Compensittion (Wieser. W.. ed.). pp. 83-45. Springer. New York.

521 Pye. V.I. Wieser. W. and Zich. M. (1976) Comp. Biochem. Ptysiol. B 54B. 1,3-20

522 Quinn, P.J., Brain. A.P.R., Slcirati, 2.2 an.l Kaltes, M. (1486) Biochim. Biophys. Icla 86.3, 21,3-223.

523 Radda, G.K. (1986) Science 233, 641-645.

524 Raederstorff. D.. Meier, C.A., Moser. U. and W.lter. P. (|Wy|) Lipids 26. 781-787.

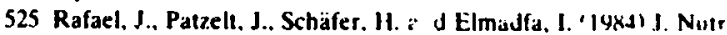
114, 255-262.

526 Rainier, S., Jain. M.K., Ramirez. F.. loannou. P.V'.. Marecek. J.F. and Wagner. R. (1979) Biochim. Rimphys. Acta 5\$8, 187-148.

527 Raison, J.K. (1973) Bioene rgetics 4. 285-309.

528 Raison, J.K. McMurchie. E.J.. Charnock, J.S. i.IJ Gilswn, R.A. (1981) Comp. Biochem. Physiol. B 69B. 169-174.

529 Rand. R.P. and Sengupta. S. (1972) Biochim. Biophys. Acta 255. 484-492.
53!) Rasmussen. (1.B.. Kishrle. J., Rokon, H. and Hesch. R.-D. (1989)

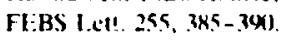

531 Ratcliff. S.I. Honsack. J.A.. Whecker, G.E. and Rore, A.H (1973) J. Cien. Microhitil. 7h. 445-444.

532 Ray. P.H.. Whitc. D.C and Brock. T.D. (1471) J. Bacteriol, I(KK, 23.235

533 Reitul, D.K. ()'Rourke, B. Foster. K.A., Hulchinum, H., Utwh. C. F. and KenI. R. L. (1486) Am. J. Physiol. 2S0, HI-Hh.

534 Reinilä. A and Ákerblom. H.K. ( $14 \times 4)$ Ulatetologla $27,347-412$.

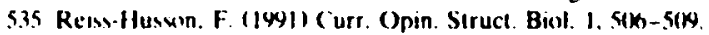

5.3h Reitz. R.C.. Thompun, J.A. and Morris. H.P. (1977) Cancer Res. 37, 56] 5 57.

\$37 Renncr. R., Innis. S.M. and (landinin. M.T. (1979) J. Nutr. I(Y. $378-3.37$.

536 Rial. E., Arćchaga, I. Sainz-de-la-Maza, E. and Nicholls. D.G. (1989) Eur. J. Bixchem. 182. 187-193.

539 Ricquier. D. Barlet. J.-P.. Garel, J. M.. Combes-George. M. and Duhois, M.P. (1983) Biochem. J. 210. 859-866.

540 Ricquier. D.. Mory. G. and Hémon, P. (1975) FEBS Lett. 53. 342-346.

541 Ricquier, D., Mory. G.. Nechad, M. and Hémon. P. (1478) J. Physiol. Paris 74, 695-7012.

542 Kidgway. G.J. and Douglas. II.C. (1964) J. Bacteriol. 76. 163-166.

54.3 Rietveld. A. Van Kemenlade. T.J.J.M.. Hak. T.. Verkleij. A.J. and De Kruijff. B. (1987) Eur. J. Bischem. 164, 137-141.

544 Riisom. T.. Johnison. S.. Hill. E.G. and Holman. R.T. (1981) J. Lah. Clin. Med. 4X. 764-775.

545 Rikans. L. 2 and Nonley, B.A. ( $14 \times 1)$ Exp. Gerontui. 16. 253-259.

Sto River. I.P.W. and Frankel. T.L. (|YX|) Br Med. Bul'. 37. $54-64$.

5.7. Ribirs, J.P.W. Sinclair, A.J. and (rauford, M.A. (1975) Nalure 258. $171-17.3$.

546 Robhlec, N.M. and Clandinin. M.T. (1944) J. Nutr. 114, 26.3-269.

549 Rohinwen. N.C.. Strey. F. and Talhert. L. (1980) Biochemistry 19. 3656-36x).

550) Rexquelin. G.. Guenot. L.. Astorg. P.O. and David. M. (1989) Lipids 21. 775-7(1).

551 Rogers. C.G. (1974) L.jpids 9. 541-547.

552 Rovio, M.. Howius. R.. Demel, R.. Wallimün, T., Eppenterger. H.M. and Nicolay, K. (|Wy|) FEBS Lett. 281, 123-124.

55.3 Rinsignol. M. Thomas, P. and Grignon. C. (1982) Biochim Biophy:. Acta to64, 195-199.

554 Rotlem. S. (1982) Curr. T(t). Mernhr. Transp. 17, 235-2n1.

555 Rotlenterk, Hi. (1485) Mol. Cell Biol. 4, 47-8.3.

55h Rottenthery. H. (1484) Methods Enzymol. 172. 6.3- 44

597 Rottenberk. H. and Hashimulc. K. (1986) Biochemistry 25. 1747. 1753.

558 Rottenherg. H.. Robertson. D.E. and Rubin. E. (1980) Lab. Invest. 42. 318-326.

559 Rottenterg. H., Rohertison. D.E. and Ruhin. E. (1985) Biochim. Biophys. Acta Xixy. 1-10.

561) Rottenberg. H.. Waring. A.J. and Kubin. E. (1984) Science 223 , 193-194.

561 Rouser. G.. Simon. G. and Kritchersky. G. (1969) Lipids 4. $549-6) 6$.

562 Rubin. E., Euaiic. D.S. and Licher. C.S. (1970) Ldb. Intesi. 23. 62, $1-627$

563 Ruggiero, F.M., Landriscina, C.. Gnoni, G.V. and Quagliariello, E. (1984) Lipids 14, 171-178.

564 Runswick. M.J.. Powell, S.J., Nyren. P. and Walker. J.E. (1987) EMEO J. 6. 1367-1373.

5h5 Russell, N.J. and Hanwood. J.L. (1919) Biochem. J. IX!, 339-345.

\$\$6 Rydström. J. and Fleischer. S. (1979) Methods Enrymol. S0. $811-816$.

567 Ryuzaki. M. (1981) Sci. Rep. Lab. Amphibian Biol., Hiroshima Univ. 5. 167-183. 


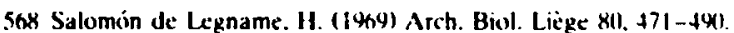
569 Sandermann, H. Jr. (1978) Biochim. Biophys. Acta 515. 209-213 570 Sankaram, M.B., Pouell. G.I.. and Marsh. D. (1489) Bioxhim. Biophys. Acta 980, 384-342

571 Sanson. A., Prak. M.. Rigatud. J.L. and Gary-Pkotx). (C. M. (197h) Chem. Phys. Lipids 17, 435-444.

572 Santiago, E., Lipez-Moralalla. N. and Sipovia. J.L. (197?,) Biochem. Binphys. Res. Cimniun. 53, 439-445.

573 Sauer. L.A. (1964) Buxhem. Biuphys. Res. Comniun. 17. $294-$ 300.

574 Schilling. R.J. and Reitz. R.C. (19ख्(1)) Biochim. Biophys. Acta 603. $26 x-277$.

575 Schlame, M., Beyer, K. Hayer-Harl, M. and Klingenterg. $M$. (1991) Eur. J. Biochem. 199, 459-466.

576 Schlame. M., Hờvàth. I.. Török. Z., Horvath, L.I. and Vigh. L. (1990) Biochim. Biophys. Acta 1(1245, 1-8.

577 Schlame. M.. Hirvaith L. and Vish. L. (1990) Biochem. J. 26.5. $79-85$.

578 Schlame, M. and Rüstow. B. (1990)) Bicchem. J. 272. 589-595.

579 Scholander. P.F.. Irving. L. and Grinnell. S.W. (1942) J. (cll. Comp. Physiol. 19. 67-7R.

580 Schreiner. G.F., Flye. W. Brunt. E., Korter. K. and Lefkowith. J.E. (1986) Science 241). 1032-1033.

581 Schudrtz. R.M. and Dayhoff. M.O. (1978) Science 149. $395-403$.

582 Secidon. J.M., Kaye. R.D. and Marsh. D. (1983) Bicchim. Bio. phys. Acta 734, 347-352.

58.3 Sedgwick, E.G., Hou, C. and Bragg, P.D. (1984) Biochim. Biophys. Acta $767,479-492$.

584 Seewald, M. and Eichinger, H.M. (1989) I Chromatugr. 469. $271-2 \times n$.

585 Scher, A.. Spiegel. II. Künker. S. and Oslage. H.J. (196.3) Felle Scifel: Anstrichmittcl 85. 295-.314.

586 Sritz, H.J., Müller, M.J. and Soboll, S. (1985) Biochem. J. 227. 149-153.

587 Sellers. E.A. and You, S.S. (195(1) Am. J. Physiol. 163. 81-91.

S88 Seluyn, M.J. (1987) Nature 33). 424-425.

S89 Sen, N. (Hulbert. A.J.) (1986) Honours Master of Science Thesis, U. Wollongong, Australia.

59) Sen, P.C. and Ray, T.K. (19*0) Biochim. Biophys. Acta. 618. 300-307.

591 Senault, C.. Yazbeck, J. Finubern, M., Porter, R.. Vincent, M. and Gallay. J. (1990) Biochim. Biophys. Acta 1023, 283-289.

542 Senne. B.C.. Swan. P.B. and Hegarty, P.V.J. (1483) Fed. Iror. 42. 1307.

593 Shaikh. N.A. and Downar. E.. (1981) Circ. Res. 49. 310-325.

594 Shapiro. I.M. and Lee. N.H. (1975) Arch. Biochem. Biophys. 170. 627-633.

S95 Shaw. M.J. and Hoch. F.L.. (1976) Life Sci. 19. 1359-1364.

5\% Shearman, M.S. and Halestrap. A.P. (19\$4) Biochem. J. 223. $673-676$.

597 Shears, S.B. (1980) J. Theor. Biol. 82, 1-13.

598 Shears. S.B. and Bronk. J.R. (1979) Biochem. J. 178, 505-507.

599 Shears, S.B. and Bronk. J.R. (198(1) J. Bicenerg. Biomembr. 12. $379-393$.

6(M) Shen. P.Y.. Coles. E. Foote. J.L. and Stenesh. J. (1970) J. Bacteriol. 103, 479-481.

601 Shively, J.M. and Benson. A.A. (196,7) J. Bacteriol. 94. 16/41683.

002 Shoffner, J.M. IV and Wallace. D.C. (1990) Adv. Hum. Genet. 19. 267-332.

603 Short, S.A. and White. D.C. (1972) J. Bacteriol. 109. 820-826.

604 Shrago, E., Shug. A.L., Sul, H., Bittar. N. and Folts, J.D. (1976) Circ. Res. Suppl. 38. 1.75-79.

60S Shrago, E. and Strieleman. P.J. (1987) World. Rev. Nutr. Diet. 53, 171-217.

606 Simun, G. and Rouser, G. (1\%9) Lipids 4, 607-614.
617) Simon. R.C. Eybel. C.E.. Galster. W. and Morrimin. P. (1971) Comp. Biochem. Physiol. B Al)B. will-6it4.

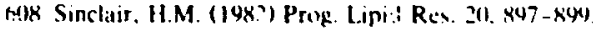

inde Skriver. L. and Thumpoun. (i.A.. Jr. (j)7b) Bickhim. Biophys. Ac1:1 431. IXI- IXS.

hill Sikrver. L. and Thumporm. (i.A.. Jr. (1474) Bieshim. Buphy Acta $572,376 \cdots 3 \times 1$.

hI! Slater. E.C. (1967) Methods Enrymol. 10) 4\$-57.

012 Smaal. E.B. Romijn. D. (jcurts van Kessel. W.S.M. De Kruiff. B. and De Gier. J. (1985) J. Lipid Res. 26. 634-637.

61.3 Smith. A.L.. (1967) Methods Enzymol. 10. $81-86$.

614 Smith. E.L., Hill. R.L.. Lehman. I.R., Lefkowitz. R.J., Handler. P. and Whilc. A. (1983) Principies of Biochemistry: General Aspects. 7th Ed., pp. 334-335, McGraw-Hill, New Yolk.

615 Smith. M.W., Col'an. Y.. Kahng. M.W. and Trump. B.F. $(19 \times())$ Biochim. Brophys. Acta 618, 192-201.

616 Smith. P.F.. Koostra. W.L. and Henrikson. C.V. (195) J. Bacteriol. 90. 282-283.

617 Sone. N. and Nichulls. P. (1984) Biochemistry 23, 6550-6554.

h18 Sone, N.. Yoshida. M.. Hirata. il. and Kayava. Y. (1975) J. Biol. Chem. 250. 7917-7923.

619 Sone. N.. Yoshida, M.. Hirata. H.. Okamoto. H. and Kagawa. Y. (1976) J. Membr. Biol. 30. 121-134.

h20) Soussi, B.. Bylund-Fellenius. A.-C., Scherstén, T. and Ängström. 1. (1990)) Biochem. J. 265, 227-232.

62I Snussi. B.. Idström, 3.-P., Scherstén. T. and Bylund-Fôllenius, A.-C. (1990) Acta Physinl. Scand. 138, 107-114.

622 Spach. P.I. and Cunningham. C.C. (1987) Biochim. Biophys. Asiu 00 : 460 16?

623 Spach. P.I., Parce, J.W. and Cunningham. C.C. (1979) Bicxhem. J. 178, 23-33.

624 Spector. A.A. and Yorek. M.A. (1985) J. Lipid Res. 26, 101510.35.

625 Spencer. T.L., See, J.K. and Bygrave. F.L. (1976) Biochim. Biophys. Acta 423, 365-373.

626 Spencer, W.A. and Dempster, G. (1921) (an. J. Biochem. Phys. iol. 40, $1705-1715$.

627 Stambough, J.L., Brighton, C.T., Iannotti, J.P. and Storey, B.T. (1981) J. Orthop. Res. 2, 235-24t.

628 Stancliff. R.C. Williams. M.A., Itsumi, K. and Pacier. L. (1969) Arch. Biochem. Biophys. 131, 629-642.

h24 Stárka. J. and Moravová, J. (1970) J. Gen. Microbiol. Łn, 251257.

630 Sierling. K. (1986) Endocrinology :19, 292-295.

631 Stewart. L.C.. Kates. M. and Smith. 1.C.P. (1988) Chem. Phys. Lipids 4\$, 177-188.

632 Stocker, W.W., Samaha. F.J. and DeGroot. L.J. (1968) Am. J. Mied. 44, 900-919.

633 Strickland. E.H. and Benson. A.A. (1960) Arch. Bicchem. Biophys. 8., 344-34s:

6.34 Strieleman, P.J., Schalinske. K.L. and Shrago, E. (1485) Biochem. Biophys. Res. Comn.un. 127, $5019-516$.

6.35 Stuhne-Sekalec, L., Wassenaar, M., Jackowski, G. and Stanacev. N.Z. (1990) Membr. Biochem. 9, 29-45.

6.3 Sul, H.S.. Shrago, E.. Goldfarh. S and Rose. F. (1970) Binchim Biophys. Acts 551. 148-156.

637 Sundin. U. (1981) Am. J. Physiol. 241, C134-C139.

638 Susin. M. and Herdson, P.B. (1967) Arch. Pathol. 83, 86-98.

639 Swanson. J.E. and Kinsella. J.E. (1986) J. Nutr. 116, 514-523.

640 Szymanski, E.S., Little, N.A. and Kritchevsky, D. (1981) Exp. Gerontol. 16. 163-169.

641 Tata, J.R. (1967) Biochem. J. 105, 783-801.

642 Tata. J.R. (1970) Biochem. J. 116. 617-630.

643 Tata, J.R., Ernster, L., Lindberg. O., Arrhenius, E., Pedersen. J. and Hedman. R. (1963) Biochem. J. 86, 408-428.

644 Taurhi, H. and Sato. T. (1980) Mech. Ageing Dev. 12, 7-14. 


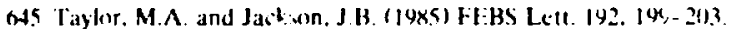

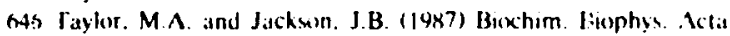
X91. 242-255

647 Teisici. J.. Prak. M. I eMassu. A.. Slewarl. I C. an I Kates. M. (144) Binchemistry 24. 54-h5.

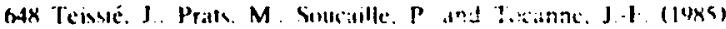
Proc. Natl Acad Sici USA 82. 3217-3221.

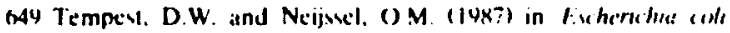
and Salmome'la nphimurium. Celiular and Molccular Bistog! INeidhardt. F.C.. ed 1. Vol 1, pp. 707-XIXI. Am. Six. Microbiol. Wishingtun.

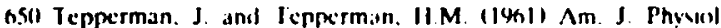
2(x). 11k9-1073.

651 Thayer, W.S and Ruhin. E. (1974) J. Bivl. Chem. 254, 7?!7 7723.

652 Thayer. W.S. and Rubin. E. (19x() Bicxilim. Biwphys. Acla X\$4. 3 ith-373.

65.3 Thomas, K.K. and Giltert. L.I. (1967) (onp. Biochem. Physiol. 21. 279-29)

o54 Thompenn. 3.A. and Reity, R.C. (1978) lipids 13. 5411-5511.

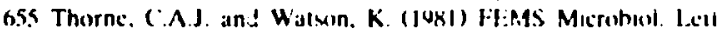
10., 137-141.

G5h Thorne. R.F.W and Bygtave, F.L. (147.3) (ancer Res. 3.3. 256.22567.

657 Tincco, J.. Bahock. R., Hincenhergs, I. Medwadimshi, B and Miljanich. P. :1978) Lipids 13, h-17.

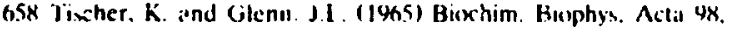
$5012-511$.

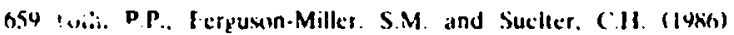
Methoxl. Eringnwi. 194. 1t-27.

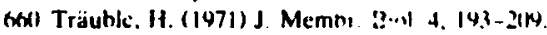

66) Träuble, H (1472) Bicomembranes 3, 14i-227

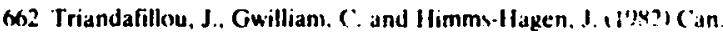
J. Biochem. 601. 530-5.37.

(x.3 Tsai. A.-1. and Palmer. G. (1986) Bi(xhini. Bi(1),hys. Acta X52. $1(x)-105$.

(64) Ulsamer. A.G.. Smith. F.R. and Korn. E.D. (1'siy) J. (ell Biol. 43. $1105-114$.

o65 Van den Bosch. H. (1982) in New Comprehensive Bicchemistry Vol. 4. Phespholipids (Hawthorne, J.N. and Ansell, G.B., eds.). pp. 313-357, Llsevier. Amsterdam.

otht Van den Thillart. G. and Merdderkolk. J. (1978) Biocitim. Bio. phys. Acta 510, 38-5I.

6h7 Van Viel, H.H.D.M.. Op den Kump. J.A.F. and Van Déenen. L.L.M (1975) Arch. Bioxhem. Biophys. 171. 55-04.

60* Vasdev, S.C.. Biro, (i.P., Nathaily, R. and Kako, K.J. (14ki)! Can. J. Bicchem. 58. 1112-1119.

toxy Vasdev, S.C., Kako, K.J. and Biro, G.P. (1979) J. Mol. (iell. Cardiol. 11. 1195-12(X).

670 Vasdev S.C.. Korecky. B.. Rastogi. R.B.. Singhal, R.L. and Kako. K.J. (1977) Can. J. Physiol. Pharmacol. 55, 1311-1314.

671 Vasilenko. I., De Kruijff. B. and Verkleij. A.J. (1982) Bickhim. Biophys. Acta 6\$4, 282-286.

6.72 Venkatesate. S., Rideout, J.M. and Simpoon. K.J. ( (1940)) Biomed. Chromatogr. 4. 234-23x.

673 Verhoeven, A.J., Kamer, P., Groen, A.K. and Tager. J.A. (1985) Biochem. J. 226, 183-192.

674 Verner, K. and Schatz, o (1988) Science 241. 1307-1313.

675 Vester, J.W. and Stadie, W.C. (1957) J. Biol. Chem. 227, 6hy676.

676 Vidal, J.C., Mclntyre, J.O., Churchill, P.. Andrew. J.A. Pihuet. M. and Fleischer, S. (1983) Arch. Birchem. Biophys. 224, 643658.

677 Videla, L.A. (1978) Clin. Sci. Mol. Med. 55. 341-347.

678 Vik, S.B., Georgevich. G. and Capaldi. R.A. (1980) Proc. Natl Acad. Sci. USA 78, 1456-1460.

679 Virji, M. and Knowles, P.F. (!978) Bioch:m. J. 169, 343-353.
hSil Vitale, J.J.. Nalaamura, M. and Hegsted. D.M. (1957) J. Bich Chem. 22x, 573-57\%.

(2) Vitorica. 3.. (\%ark. A. Machado. A. and Saltrúclequi. 3. (1985) Mech. Ageing Lev. 29. 255-2tot.

ok: Vorbech. M.L.. Martin. A.P.. Long. J.W. Jr.. Smith. J $M$. and (Jrr, KR. Ir. (1UK2) Arch. Biechem Biophys. 217, 351 361.

hol Vorteck. M.L.. Maltin. A.P.. Park. J.K.J. and Tinnend. J.F. 114X2I Arch. Bickem. Biophws. 214. 67-74.

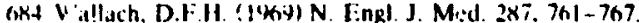

fos W.irhurg. O. (1430) The Metabulism of Tumors (Dickens. F. !rar. l. $327 \mathrm{pp}$. Richard Sinith. New Haven.

tokh Wal ng. $\wedge$ J., Roll!anherg. H. Ohnishi. T. and Ruhin. E. (IUkI) Pric. Natl. Acad. Sci. USA 78. 2582-258h.

\&:? Watmon. K. (IUAa) in Membrane Fluidity. Biophysical Tech. niques and (ellular Regulation (Kates, M. and Kuksis, A., eds.). pn. 344-36.3. Humana. Clifion.

E.KK Watson. K. Bertoli. E. and Griffiths. D.E. (1975) Biochem. J. 146. $4(1)$ - 4117 .

ה84 Watson. K. and Rose. A.H. (1480) J. Gen. Microhiol. 117 , $225-233$

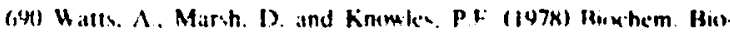
phys. Res. Commull. $81 . \$ 113-4(K)$.

(19) Wedver. T. I... Patrick. M.A an J Dugion. P.R (1975) J Bacteriol. 124. (x)2-6xis

692 Webh. A.C. and Smith. I.D. (1977) I24. B161. 56. 214. 225.

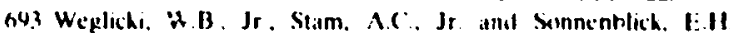
(1970) J. Mol. ( :II. Cardini 1. 131-142

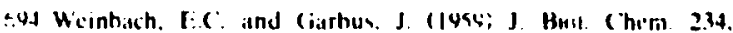
$+12-+17$.

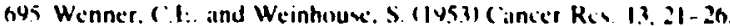

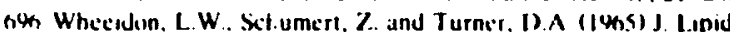
Res. h. $4 \times 1-4 \times 4$

697 White. D.A. (1973) if Form and Function of Phospholipids (Ancell. G. B.. Hawthorne, J.N. and Daweon, R.M. ., c.ds.) BBA Litian 3. $441-4 \times 2$.

698 White. D.C (!96x) J. Bacteriol th. 1159-11701

649 White. DC. and Fierman. F.E. (146K) J. Basteriol. 95, 2198$22(Y)$.

7(h) Wies!ander, $\AA$.. Chrisians.en. A. Rilfors. L. and Lindhlom. (; (19 14$)$ ) Biochemistry 14, 3n501-3055.

701) Wilkinwon. B.I. Morman. M.R. and White. D.C. (!'37) J. Bacte. riwl. 112. 12XK-1244.

712 William.. (.M and E:His. R. 11985) Binsi. Rep. S. 175-184.

7013 Williams. M.A. Stancliff, R.C.. Packer, L. and Kicith, A.D. (1972) Bicchim. Bisphys. Acts 267, 414-456.

$7(14$ Williums. R.J.P. (146)1) J. Theor. Biol ?(1. 1-17.

70 S Winder. W.W.. Baldwin. K.M.. Terjung. R.L. and Heolluszy, J.O. (1975) Am. J. Physiol. 228. 1.341-1345.

7(K Winder. W.W. and Holloszy. J.O. (1U77, Am. I Physiol. 232. 180)-184.

707 Winkler, L.. Buanga, N.-F. and Girelec, r. (1971) Biacium. Bimphys Actia 2.31, 535-5.36.

708 Withers. K.W. and Hulbert, A.J. (1987) Nutr. Res. 7. $11.34-1150$.

$7(K)$ Wlodawer, P. and Boguslauska. Z. (196x) (omp. Biochem. Physiol. 26. 597-611.

710 Wodtke. E. (1973) in Effects of Temperature on Ectothermic Organisms. Ecological Implications and Mechanisms of Compensation (Wieser. W.. ed.), pp. 97-105, Springer, New York.

711 Wodtke. E. (1974) J. Comp. Physiol. 91, 277-307.

71? Wodtke. E. (1978) Biochim. Biuphys. Acta 529. 280- 291.

713 Wodike. E. (1961) Bıochim. Biophys. Acta 641). 698-704.

714 Wodtke. E. (1961) Biochim. Biophys. Acta 640, 710-720).

7:S Woesc. C.R. (1987) Microbiol. Rev. \$1. 221-271.

716 Wolff, R.L. (1988) Reprod. Nutr. Dévelop. 28, 1489-1507.

717 Wolff. R.L.. Comte, N. and Entıessangles, B. (1984) Rev. Fr. Corps Gras 31, 161-170. 
718 Wolft. R.L.. Comber. N. and Entressangles. B. (1985) Res. Fr Corps Gras 32. 295-244.

719 Wolft. R.L... Combe. N. and Entressangles B. (1985.51 I inik 2il. MIX-014.

72) Wolff. Ki... and Dumant. T.C (| $4 \times 7)$ Rev. Fi (iorm (itas 34. $4113-4116$.

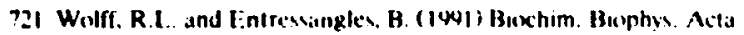
$116 \times 2.13 \%-142$

72 Wined. R. (1075) Linids (1). 73n-715

72.3 Wonvward. J.A. and Saggerw:n. F.D. (14xh) Biochem. J. 23x. 39.5- H13.

724 Worcester. N.A. Bruck dorfer. K.R.. Hallinan. T. Wilkins. A.J.. Mann. J.A. and Yudkin. J. (1974) Br. J. Nutr. 41. 239-252.

725 Wuthier. R.E. (1\%8) J. Lipid Res. 9, (x)-7\%.

726 Wuthier. R.E. (1975) Piochim. Biophys. Acta HiW. 128-143.

727 Yaffe. S. Gild, A. and Sampugna. 3. (14:40) J. Nutr. 110. 24(4)-2446.

72x Yamamoto, A., Isszaki, M. Hirayama, K. and S:sk.oi V acksi J. Lipid Res. 6. 295-30)

729 Yamuoka. S. (Irade. R. and Kito, M. (1988) J. Nutr. IIR. 29)

7.3) Yamauchi, T.. Ohki. K. Maruyama. H. and No/awd. Y. (|48|) Biochim. Biophys. Acla (49, 385-392.

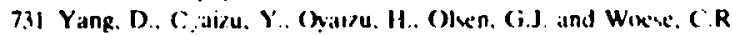
(I\%xS) Prox. Nall. Acad. Sci. USA K2. +2+3-4+47.

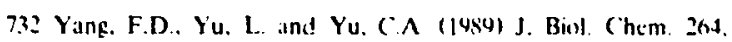
Kul-SUS.

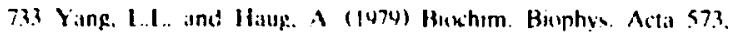
$3(18-.320$.

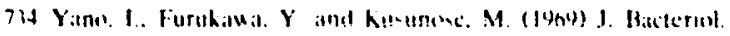
4X. 124-1311.

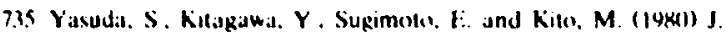
Bicchein. Tokyo \$7. 1511-1517.

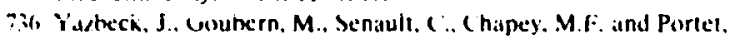
R. (14XY) (iumn. Biochem. Physiol. 44A. 273-27\%

737 Young. V.R. $(1974)$ Fed. Prox. 3x. 1444-2(3(x).

7.36 Yu. B.P.. Bertrand, H.A. and Masort). E.J. (1)k(1) Metaholism Clin. Exp. 29, 4.3k-444.

734 Yu. L.. Yu. C.A. and king. T.E. (1476) J. Biol. Chem. 253, $2657-266.3$

740 Yurkowaki. M. and Walker. B.L. (1970) Can. J. Physiol. Pharmacol. $48,6.31-6.39$.

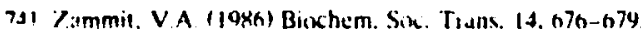

742 7.hu, Y.-P., Su, Z.-W. and I.i. C.-11. (1989) J. Natl. Cances Inct. 81. 1.3) $2-1.31 \mathrm{~K}$

74.3 Zexeller. R.A and W(u)d. R. (1484) I inids 19. 539.538.

741 Zilkicuska, A. Zahlocka. B. Dusninski. J. and Wojtcrak. I.. (1489) Arch. Bicchem. Biophys. 275. 5(4)-54). 